\title{
Characterization of human breast cancer cells affected by coculture conditions and kisspeptin-10
}

\author{
Doctoral Thesis \\ In partial fulfillment of the requirements for the degree \\ "Doctor rerum naturalium (Dr. rer. nat.)" \\ in the Molecular Medicine Study Program \\ at the Georg-August University Göttingen
}

submitted by

Elke Ziegler

born in Darmstadt, Germany

Göttingen, March 2013 


\section{Thesis Committee}

\section{Supervisor}

Prof. Dr. Carsten Gründker

Department of Gynecology and Obstetrics

Laboratory for Molecular Gynecology

University Medicine Göttingen

\section{Second member of the thesis committee}

Prof. Dr. Matthias Dobbelstein

Department of Molecular Oncology

Georg-August University Göttingen

Third member of the thesis committee

Prof. Dr. Sigrid Hoyer-Fender

Department of Developmental Biology

Georg-August University Göttingen

Date of Disputation: 


\section{Affidavit}

I hereby declare that my doctoral thesis entitled "Characterization of human breast cancer cells affected by coculture conditions and kisspeptin-10" has been written independently with no other sources and aids than quoted.

Elke Ziegler

Göttingen, March 2013 


\section{Publications}

E. Ziegler, T. Olbrich, G. Emons, and C. Gründker. Antiproliferative effects of kisspeptin-10 depend on artificial GPR54 (KISS1R) expression levels. Oncol Rep, 29(2):549-554, Feb 2013.

T. Olbrich, E. Ziegler, G. Türk, A. Schubert, G. Emons, and C. Gründker. Kisspeptin-10 inhibits bone-directed migration of GPR54-positive breast cancer cells: Evidence for a dose-window effect. Gynecol Oncol, 119(3):571-578, Dec 2010.

\section{Congress participations}

126th Meeting of the North German Society for Gynecology and Obstetrics 2010, Schwerin (Germany)

Poster: E. Ziegler, T. Olbrich, G. Emons, and C. Gründker. Der Einfluss von Kisspeptin-10 auf die Expression migrationsrelevanter Gene beim Mammakarzinom (Influence of kisspeptin-10 on gene expression of genes involved in migration in breast cancer).

34th Annual Meeting of the German Society for Cell Biology 2011, Bonn (Germany) Poster: E. Ziegler, T. Olbrich, G. Emons, and C. Gründker. EMT marker expression in breast cancer cells during bone-directed migration.

30th German Cancer Congress of the German Cancer Society 2012, Berlin (Germany)

Poster: E. Ziegler, G. Emons, and C. Gründker. Kisspeptin-10 reduces tumor growth and metastasis in a breast cancer model in vivo.

128th Meeting of the North German Society for Gynecology and Obstetrics 2012, Kiel (Germany)

Poster: E. Ziegler, T. Olbrich, G. Emons, and C. Gründker. Die antiproliferative Wirkung von Kisspeptin-10 ist abhängig vom GPR54-Expressionsstatus (Antiproliferative effects of kisspeptin-10 depend on GPR54 expression levels). 


\section{Contents}

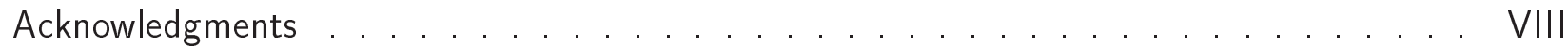

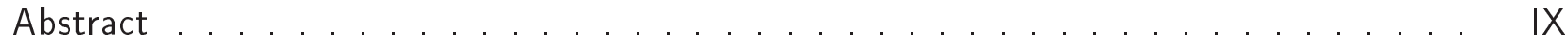

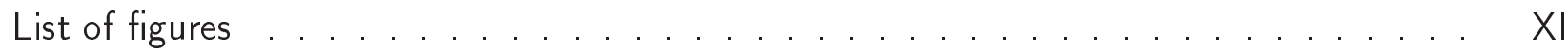

List of tables . . . . . . . . . . . . . . . . . . . . . X XII

List of abbreviations . . . . . . . . . . . . . . . . . . . XIII

1 Introduction . . . . . . . . . . . . . . . . . . . . . . . . 1

1.1 Breast cancer . . . . . . . . . . . . . . . . . . . . . . . . . 1

1.1.1 Epidemiology and etiology . . . . . . . . . . . . . . . . . . 1

1.1.2 Classification and etiopathology . . . . . . . . . . . . . . . . 1

1.1 .3 Therapy . . . . . . . . . . . . . . . . 3

1.2 Metastasis . . . . . . . . . . . . . . . . . . . . . 3

1.2.1 Metastasis cascade . . . . . . . . . . . . . . . . . . . 4

1.2.2 Progression of metastasis . . . . . . . . . . . . . . . 5

1.2.3 Motility mechanisms of cells . . . . . . . . . . . . . . . 6

1.2.4 Microenvironmental dependency of metastatic settlement . . . . . . . 8

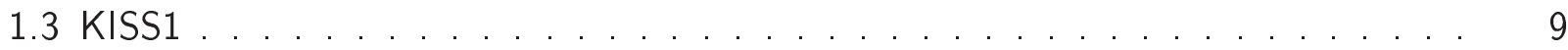

1.3.1 KISS1 and its receptor GPR54 . . . . . . . . . . . . . . . . 9

1.3.2 Cell motility and metastasis affected by kisspeptins . . . . . . . . . . . 10

1.3 .3 Kisspeptin analogs . . . . . . . . . . . . . . . . . . . 11

1.4 Outline of the thesis . . . . . . . . . . . . . . . . . . 11

2 Material and methods . . . . . . . . . . . . . . . . . . . . 13

2.1 Material . . . . . . . . . . . . . . . . . . . . . . . . . . . . . 13

2.1.1 Biological substances, chemicals, commercial reagents and kits . . . . . . 13

2.1 .2 Buffers, solutions and media . . . . . . . . . . . . . . . 16

2.1 .3 Enzymes . . . . . . . . . . . . . . . . . . . . . . 17

2.1 .4 Oligonucleotides . . . . . . . . . . . . . . . . . . . . . . . . . . . 18

2.1 .5 Antibodies . . . . . . . . . . . . . . . . . . . . . . . . 19

2.1 .6 Biological material . . . . . . . . . . . . . . . . . . . . . 19

2.1 .7 Animals . . . . . . . . . . . . . . . . . . . . . . . 20

2.1 .8 Consumable supplies . . . . . . . . . . . . . . . . . . . . 20

2.1 .9 Equipment . . . . . . . . . . . . . . . . . . 20 
2.2 Methods . . . . . . . . . . . . . . . . . . . . . . . . . . . . . . 21

2.2.1 Cell biology . . . . . . . . . . . . . . . . . . . . . . . . 21

2.2 .2 Cocultivation . . . . . . . . . . . . . . . . . . . . . . . 21

2.2.3 Molecular biology . . . . . . . . . . . . . . . . . . . . . . . 23

2.2.4 Protein biochemistry . . . . . . . . . . . . . . . . . . 28

2.2 .5 Proliferation assay . . . . . . . . . . . . . . . . . 30

2.2 .6 Animal experiments . . . . . . . . . . . . . . . . . . . 30

2.2 .7 Statistical analysis . . . . . . . . . . . . . . . . . . 32

3 Results . . . . . . . . . . . . . . . . . . . . . . . . . 33

3.1 Morphology and motility marker expression of breast cancer cells . . . . . . . . 33

3.1.1 Morphological characteristics of breast cancer cells . . . . . . . . . . . 33

3.1 .2 EMT marker expression . . . . . . . . . . . . . . . . . . . 34

3.2 Invasion of breast cancer cells . . . . . . . . . . . . . . . . . . . . 35

3.2 .1 Invasive properties of breast cancer cells . . . . . . . . . . . 35

3.2.2 Progression of bone-directed invasion . . . . . . . . . . . . 38

3.2.3 Influence of $\mathrm{TGF} \beta_{1}$ treatment on breast cancer cell invasion . . . . . . . 42

3.2.4 Motility marker expression affected by cocultivation and $\mathrm{TGF} \beta_{1} \ldots \ldots 44$

3.3 Gene expression in MCF-7 cells during cocultivation . . . . . . . . . . . 46

3.3 .1 Microarray analysis . . . . . . . . . . . . . . . . . . . 46

3.3.2 Expression of genes involved in cell motility processes . . . . . . . . . . 48

3.4 Effects of kisspeptin-10 and analogs on bone-directed invasion . . . . . . . . 49

3.4.1 Invasion of breast cancer cells treated with kisspeptin-10 . . . . . . . 50

3.4.2 Invasion of breast cancer cells treated with a kisspeptin-10 analog . . . . . 51

3.5 Effects of kisspeptin-10 in vivo . . . . . . . . . . . . . . . . . . 52

3.5 .1 MDA-MB-231 breast cancer xenograft . . . . . . . . . . . . 52

3.5 .2 HCC 1806 breast cancer xenograft . . . . . . . . . . . . . . . 52

3.5 .3 MDA-MB-435s breast cancer xenograft . . . . . . . . . . 55

3.6 Effects of kisspeptin-10 on proliferation . . . . . . . . . . . . . 57

3.6.1 GPR54 expression in breast cancer cells and transfected cells . . . . . . . 58

3.6.2 Cell growth under kisspeptin-10 treatment . . . . . . . . . . . 61

4 Discussion . . . . . . . . . . . . . . . . . . . . . . . . . . . . . . . . 64

4.1 Coculture effects on breast cancer cells . . . . . . . . . . . . . . . 64

4.1.1 Classification of breast cancer cell lines . . . . . . . . . . . . . 64

4.1 .2 Invasive and migratory behavior . . . . . . . . . . . . . . . . 66

4.1 .3 Gene expression . . . . . . . . . . . . . . . . . . . 68

4.2 Kisspeptin-10 and breast cancer . . . . . . . . . . . . . . . . . 73

4.2 .1 In vitro effects on invasion . . . . . . . . . . . . . . 73

4.2 .2 In vivo effects on tumor growth and metastasis . . . . . . . . . . . 74 
4.2.3 In vitro effects on proliferation . . . . . . . . . . . . . . . . . . . 76

5 Summary and conclusions . . . . . . . . . . . . . . . . . . . . . . . . . . . 79

Bibliography . . . . . . . . . . . . . . . . . . . . . . . . 80

Appendix . . . . . . . . . . . . . . . . . . . . . . . . . 91

A1 Sample heatmap of the microarray data . . . . . . . . . . . . . . . . . . . . . . . . . 92

A2 Candidates list of the microarray data . . . . . . . . . . . . . . . . . . . . . . . . . 93

Curriculum Vitae . . . . . . . . . . . . . . . . . . . . . . . . . . . . . . . . . . . . . . . . . . . 103 


\section{Acknowledgments}

This work was conducted in the Laboratory for Molecular Gynecology, Department of Gynecology and Obstetrics, University Medicine Göttingen. I would like to thank Prof. Dr. Carsten Gründker as my supervisor and Prof. Dr. Günter Emons as head of the department for giving me the opportunity to work on this project. I also want to acknowledge my thesis committee members Prof. Dr. Matthias Dobbelstein and Prof. Dr. Sigrid Hoyer-Fender for their interest in my project and their input during the thesis committee meetings.

I would like to thank Dr. Antje Schubert, Diana Rubel and Dr. Teresa Olbrich for their motivating support, our constructive dialogs and the time together in the laboratory and our office. I want to acknowledge all members of the laboratory for their technical assistance.

In addition, I want to acknowledge the Molecular Medicine PhD program for the administrative support and the Deutsche Krebshilfe - Dr. Mildred Scheel Stiftung for funding this project.

I thank my family for their infinite support over the years and my husband for encouraging me anytime and for his understanding in times, when I was more married with my lab. Thank you Jan. 


\section{Abstract}

Breast cancer is the most common type of cancer in women. Lethality is mainly attributed to a severe metastatic spread in patients. Research in new therapeutic treatments demands the understanding of the metastatic processes and the interaction of tumor cells with the distant organ microenvironment. Therefore, a coculture cell system was studied on relations between human breast cancer cells and osteoblast-like osteosarcoma cells mimicking an important metastasis environment in bone. Kisspeptin-10, a peptide derived from the metastasis suppressor gene KISS1, interferes within this cell system by inhibition of invasion. An influence of kisspeptin-10 in vivo was further investigated to evaluate its effect as a possible therapeutic option in the metastatic breast cancer disease.

Human breast cancer cells were classified by morphology, motility marker expression and their individual invasiveness. In coculture, epithelial-like cells showed a dramatic increase in invasion. Microarray gene analysis revealed potential factors of cell motility processes and immune response mechanisms, which were regulated by the interaction of breast cancer cells and osteosarcoma cells. An involvement of EMT was shown partially by upregulation of mesenchymal gene markers without an altered epithelial marker expression. CXCL12 was identified as one important factor in invasive progression: the "coculture effect" may trigger invasion by an autocrine CXCL12/CXCR4 loop. Factors as EGF and interferons are possible new targets of investigations on the interaction of breast cancer cells and osteosarcoma cells.

Kisspeptin-10 was studied in breast cancer xenografts for an influence on metastatic spread and tumor growth. Treatment with the peptide inhibited tumor growth. But investigations on cell proliferation in vitro revealed no antiproliferative effect. The role of kisspeptin-10 in angiogenetic processes is an interesting option as possible mechanism leading to decreased tumor size in treated animals. A conclusion could not be offered on an antimetastatic effect of kisspeptin-10 as possible therapeutic option in breast cancer based on the poor metastasis model in mice. 


\section{List of figures}

1.1 Metastasis cascade . . . . . . . . . . . . . . . . . . . . . . . . . . . 4

1.2 Mechanisms of cell invasion . . . . . . . . . . . . . . . . . . . 7

2.1 In vitro model for invasion studies under coculture conditions . . . . . . . . . 22

2.2 In vitro model for migration studies under coculture conditions . . . . . . . . 23

3.1 Morphology of breast cancer cells . . . . . . . . . . . . . . . . . . 34

3.2 Expression of EMT marker in breast cancer cell lines . . . . . . . . . . . 35

3.3 Invasive behavior of breast cancer cells . . . . . . . . . . . . . 36

3.4 Invasion of breast cancer cells affected by cocultivation with osteosarcoma cells . 37

3.5 Invasive characteristics of MDA-MB-231 cells in coculture . . . . . . . . . 38

3.6 Invasive characteristics of MDA-MB-435s cells in coculture . . . . . . . . . 39

3.7 Invasive characteristics of HCC 1806 cells in coculture . . . . . . . . . . 39

3.8 Invasive characteristics of MCF-7 cells in coculture . . . . . . . . . . . 40

3.9 Migratory characteristics of MCF-7 cells in coculture . . . . . . . . . . 41

3.10 Invasion of MDA-MB-231 cells treated with $\mathrm{TGF} \beta_{1} \ldots \ldots \ldots . \ldots . . \ldots 42$

3.11 Invasion of $\mathrm{HCC} 1806$ cells treated with $\mathrm{TGF} \beta_{1} \ldots \ldots \ldots . \ldots 43$

3.12 Invasion of MCF-7 cells treated with $\mathrm{TGF} \beta_{1} \ldots \ldots . \ldots . \ldots 43$

3.13 Expression of epithelial marker in breast cancer cells treated with $\mathrm{TGF} \beta_{1} \ldots .44$

3.14 Expression of mesenchymal marker in breast cancer cells treated with $\operatorname{TGF} \beta_{1} \ldots 45$

3.15 Heatmap of genes most affected by cocultivation . . . . . . . . . . . 47

3.16 Gene expression in breast cancer cells affected by cocultivation . . . . . . . . . . 49

3.17 Invasion of HCC 1806 cells treated with KP-10 . . . . . . . . . . 50

3.18 Invasion of HCC 1806 cells treated with DKP-10 . . . . . . . . . 51

3.19 Tumor growth in xenografts with HCC $1806 \ldots \ldots . \ldots . \ldots . . \ldots 53$

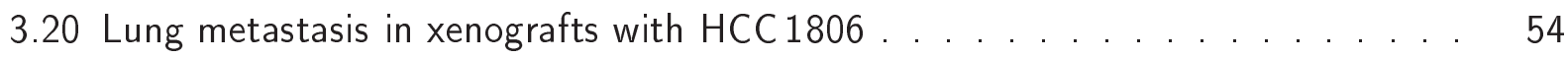

3.21 Circulating tumor cells in xenografts with HCC $1806 \ldots \ldots \ldots 55$

3.22 Tumor growth in xenografts with MDA-MB-435s . . . . . . . . . . 56

3.23 Lung metastasis in xenografts with MDA-MB-435s . . . . . . . . . . . 57

3.24 GPR54 expression in breast cancer cell lines . . . . . . . . . . . . . . 58

3.25 GPR54 mRNA expression . . . . . . . . . . . . . . . . . . . . . . . . 59 
3.26 GPR54 protein expression . . . . . . . . . . . . . . . . . . . . . 59

3.27 GPR54 mRNA expression in B35 mGPR54 clones . . . . . . . . . . . . . . . 60

3.28 GPR54 protein expression in B35 mGPR54 clones . . . . . . . . . . . . . . 61

3.29 Proliferation of breast cancer cells treated with KP-10 . . . . . . . . . . . . . 62

3.30 Proliferation of B35 mGPR54 clones treated with KP-10 . . . . . . . . . . . 63 


\section{List of tables}

1.1 Intrinsic subtypes of breast cancer . . . . . . . . . . . . . . . . 2

2.1 Biological substances . . . . . . . . . . . . . . . . . . . . . . . . . . 13

2.2 Chemicals . . . . . . . . . . . . . . . . . . . . . . . . . . . . . . . . . 14

2.3 Commercial reagents and kits . . . . . . . . . . . . . . . . 15

2.4 Buffers and solutions . . . . . . . . . . . . . . . . . . . . . . . . . 16

2.5 Media . . . . . . . . . . . . . . . . . . . . . . . . . 17

2.6 Enzymes . . . . . . . . . . . . . . . . . . . . . . . . . . . . . . 17

2.7 Oligonucleotides . . . . . . . . . . . . . . . . . . . . . . . . . . . . 18

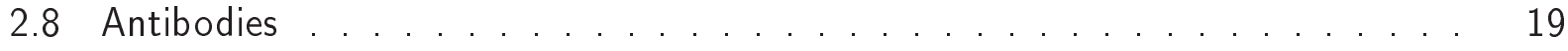

2.9 Human breast cancer cell lines . . . . . . . . . . . . . . . . . . . . . . . . . . 19

2.10 Osteosarcoma cell lines . . . . . . . . . . . . . . . . . . . . . . . . . . 19

2.11 Transfected cell clones . . . . . . . . . . . . . . . . . . . . . 20 20

2.12 Animals . . . . . . . . . . . . . . . . . . . . . . . . . . 20 20

2.13 Equipment . . . . . . . . . . . . . . . . . . . . 20

2.14 Composition for two gels . . . . . . . . . . . . . . . . . . . . . . . . 29

3.1 Classification of genes most affected by cocultivation . . . . . . . . . . . 48 


\section{List of abbreviations}

\begin{tabular}{|c|c|}
\hline Ala & alanine \\
\hline APS & ammonium persulfate \\
\hline Arg & arginine \\
\hline Asn & asparagine \\
\hline BRCA & breast cancer (proteins) \\
\hline BSA & bovine serum albumin \\
\hline BW & body weight \\
\hline $\mathrm{Ca}^{2+}$ & calcium ion \\
\hline CAF & cancer-associated fibroblast \\
\hline CAT & collective to amoeboid transition \\
\hline CD44 & cluster of differentiation 44 \\
\hline $\mathrm{CDH} 1$ & cadherin 1 , epithelial cadherin \\
\hline $\mathrm{CDH} 2$ & cadherin 2 , neuronal cadherin \\
\hline cDNA & complementary DNA \\
\hline CIS & carcinoma in situ \\
\hline $\mathrm{CO}_{2}$ & carbon dioxide \\
\hline $\mathrm{COX}-2$ & cyclooxygenase 2 \\
\hline CSF1 & colony stimulating factor 1 \\
\hline CTC & circulating tumor cell \\
\hline CTGF & connective tissue growth factor \\
\hline CXCL12 (SDF-1) & chemokine ( $\mathrm{C}-\mathrm{X}-\mathrm{C}$ motif) ligand 12 (stromal cell derived factor 1$)$ \\
\hline CXCR4 & chemokine ( $\mathrm{C}-\mathrm{X}-\mathrm{C}$ motif) receptor 4 \\
\hline DCIS & ductal carcinoma in situ \\
\hline DKP-10 & DK6-Kisspeptin-10 \\
\hline DMEM & Dulbecco's Modified Eagle Medium \\
\hline DMSO & dimethyl sulfoxide \\
\hline DNA & deoxyribonucleic acid \\
\hline dNTPs & deoxyribonucleotides \\
\hline (D)PBS & phosphate buffered saline \\
\hline DTC & disseminated tumor cell \\
\hline
\end{tabular}




\begin{tabular}{|c|c|}
\hline DTT & dithiothreitol \\
\hline ECM & extracellular matrix \\
\hline EDTA & ethylenediaminetetraacetic acid \\
\hline EGF & epidermal growth factor \\
\hline EMT & epithelial to mesenchymal transition \\
\hline ER & estrogen receptor \\
\hline $\mathrm{ERK} 1 / 2$ & extracellular signal regulated kinases $1 / 2$ \\
\hline ET1 & endothelin 1 \\
\hline $\mathrm{EtOH}$ & ethanol \\
\hline FAK & focal adhesion kinase \\
\hline FC & fold change \\
\hline FCS & fetal calf serum \\
\hline FGF & fibroblast growth factor \\
\hline FN1 & fibronectin 1 \\
\hline Gly & glycine \\
\hline GPR & G-protein coupled receptor \\
\hline GRK2 & GPR serine/threonine kinase 2 \\
\hline GSK3 $\beta$ & glycogen synthase kinase $3 \beta$ \\
\hline $\mathrm{HCl}$ & hydrochloric acid \\
\hline hDNA & human deoxyribonucleic acid \\
\hline HER2 & human epidermal growth factor receptor 2 \\
\hline HGF & hepatocyte growth factor \\
\hline $\mathrm{H}_{2} \mathrm{O}$ & water \\
\hline $\mathrm{H}_{2} \mathrm{O}_{2}$ & hydrogen peroxide \\
\hline IFIT & interferon induced protein with tetratricopeptide repeats \\
\hline $\mathrm{KCl}$ & potassium chloride \\
\hline KP-10 & kisspeptin-10 \\
\hline L7 & ribosomal protein $\mathrm{L} 7$ \\
\hline LCIS & lobular carcinoma in situ \\
\hline IGF & insulin-like growth factors \\
\hline Leu & leucine \\
\hline MAP & mitogen activated protein \\
\hline MAT & mesenchymal to amoeboid transition \\
\hline mDNA & murine deoxyribonucleic acid \\
\hline MEM & minimum Essential Medium \\
\hline $\mathrm{MeOH}$ & methanol \\
\hline mGPR & murine G-protein coupled receptor \\
\hline MMP & matrix metalloproteinase \\
\hline
\end{tabular}




\begin{tabular}{|c|c|}
\hline $\mathrm{NaCl}$ & sodium chloride \\
\hline OAS & 2'-5'-oligoadenylate synthetase \\
\hline $\mathrm{NaOH}$ & sodium hydroxide \\
\hline PAGE & polyacrylamide gel electrophoresis \\
\hline PARP9 & poly (ADP-ribose) polymerase 9 \\
\hline PCR & polymerase chain reaction \\
\hline PDGF & platelet derived growth factor \\
\hline Phe & phenylalanine \\
\hline $\mathrm{PIP}_{2}$ & phosphatidylinositol 4,5-bisphosphate \\
\hline PLC & phospholipase C \\
\hline PR & progesterone receptor \\
\hline PTHrP & parathyroid hormone-related protein \\
\hline qPCR & quantitative polymerase chain reaction \\
\hline Rac & Ras-related $\mathrm{C} 3$ botulinum toxin substrate \\
\hline RANKL & receptor activator of nuclear factor kappa-B ligand \\
\hline Rho & Ras homolog gene family \\
\hline RNA & ribonucleic acid \\
\hline ROCK & Rho-associated protein kinase \\
\hline S100A4 (FSP1) & S100 calcium binding protein A4 (fibroblast-specific protein-1) \\
\hline SDS & sodium dodecyl sulfate \\
\hline SEM & standard error of the mean \\
\hline SER & serine \\
\hline SerpinE2 & serpin peptidase inhibitor, clade $\mathrm{E}$, member 2 \\
\hline SPARC (ON) & secreted protein, acidic, cysteine-rich (osteonectin) \\
\hline STAT1 & signal transducer and activator of transcription 1 \\
\hline TACC1 & transforming acidic coiled-coil containing protein 1 \\
\hline TAM & tumor associated macrophage \\
\hline TBE & Tris-borate-EDTA buffer \\
\hline TBS & tris buffered saline \\
\hline TBST & tris buffered saline tween \\
\hline TCF21 & transcription factor 21 \\
\hline TEMED & tetramethylethylenediamine \\
\hline TGF $\beta_{1}$ & transforming growth factor $\beta_{1}$ \\
\hline TJP1 (ZO1) & tight junction protein 1 (zona occludens 1 ) \\
\hline TNF & tumor necrosis factor \\
\hline Trp & tryptophan \\
\hline Tyr & tyrosine \\
\hline UV & ultraviolet light \\
\hline
\end{tabular}


VCAM-1

VEGF

VIM

YWHAZ vascular cell adhesion protein 1

vascular endothelial growth factor

vimentin

14-3-3 protein zeta/delta 


\section{Introduction}

\subsection{Breast cancer}

\subsubsection{Epidemiology and etiology}

Breast cancer is the most occurrent type of cancer in women with an incidence of $23 \%$ of all cancers in the female population worldwide in 2008. Over the last decades, mortality rates were continuously decreasing to approximately 6-19 per 100,000 cases by improved therapies and an optimized screening especially in the developed countries [Ferlay et al., 2010]. In Germany, about 72,000 women contract with breast cancer every year and the mortality was about 17,000 in 2008. Most of the patients are of 60-70 years of age, when cancer was diagnosed. The absolute 5-years overall survival is about $78 \%$ [RKI. Robert Koch-Institut, 2012].

The main risk factors for the disease are an older age, an early age at menarche and a late age at menopause. Childlessness or age at first birth of 40 years and more are also associated with breast cancer. In addition, there is evidence that breast density is a strong risk factor. The risk of diagnosis raises by an extended use of hormone-replacement therapies in the climacteric period, whereas the risk by using oral contraceptives is almost reversible after ten years of stopping the taking. General obesity together with low physical activity, alcohol consumption and smoking seems to be involved in a higher risk of breast cancer diagnosis. Genetic factors as mutations in the breast cancer (BCRA) genes are involved in less than $10 \%$ of the cases. Having relatives suffering from breast cancer is another risk factor for the disease [WHO. World Health Organization, 2003, Veronesi et al., 2005, RKI. Robert Koch-Institut, 2012].

\subsubsection{Classification and etiopathology}

Breast cancer is classified by its histopathologic appearance in carcinoma in situ (CIS) and invasive carcinoma. CIS is differentiated in ductal (DCIS) and lobular (LCIS) depending on its origin. Both types are associated with the invasive kind of breast cancer and are considered as precancerous. 
Invasive carcinoma is defined as a malignant tumor penetrating partly or completely the basement membrane of the site of origin [WHO. World Health Organization, 2002].

Prognosis and treatment of patients suffering from breast cancer is addicted to time-dependent variables influencing tumor stage and intrinsic characteristics related to the inherent biology of the individual tumor. The stage of the tumor is characterized by the histological tumor size ( $T$ ) and the existence and dimension of lymph node $(N)$ and systemic metastasis (M) classified as TNM status. The intrinsic aspects describe the histological grade by evaluating tubule and gland formation, nuclear pleomorphism and mitotic counts (grade 1: well differentiated, grade 2: moderately differentiated, grade 3: poorly differentiated), the histological tumor type and molecular properties as receptor status of the hormone and growth factor receptors, estrogen receptor (ER), progesterone receptor (PR) and human epidermal growth factor receptor 2 (HER2), and the presence of Ki-67 as a marker for cell proliferation [WHO. World Health Organization, 2002, Veronesi et al., 2005]. Knowledge of these factors enables a classification by the intrinsic subtypes of breast cancer in luminal A, luminal B (HER2 positive/negative), HER2 positive (non-luminal) and triple negative (ductal)/basal-like (see table 1.1 ).

\begin{tabular}{|l|lll|}
\hline Intrinsic subtype & Definition & & \\
\hline \hline luminal A & ER and/or PR positive & HER2 negative & Ki67 low \\
\hline luminal B (HER2 negative) & ER and/or PR positive & HER2 negative & Ki67 high \\
\hline luminal B (HER2 positive) & ER and/or PR positive & HER2 overexpressed & any Ki67 \\
\hline HER2 positive (non-luminal) & no ER and/or PR & HER2 overexpressed & \\
\hline triple negative (ductal)/basal-like & no ER and/or PR & HER2 negative & \\
\hline
\end{tabular}

Table 1.1: Intrinsic subtypes of breast cancer

Definitions of the intrinsic subtypes of breast cancer by Goldhirsch et al. 2011, modified.

Lethality is mainly depending on the grade of the tumor showing a $90 \%$ death rate within eight years of diagnosis in patients with grade 3 tumors, within 13 years in patients with grade 2 tumors and within 30 years in patients with grade 1 tumors. A less differentiated tumor seems to be an important factor for a systemic metastatic disease and the risk of death is mainly based on the extend of metastasis rather than on the characteristics of the primary tumor [WHO. World Health Organization, 2002].

The breast cancer disease is characterized by a high rate of metastases. The primary tumor is the second common primary cancer with more than $11 \%$ of all cancers and causes the most metastatic sites with up to $24 \%$ of all metastases. The ratio of metastases per primary tumor is more than five. The preferred organs for metastasis are lymph nodes, lung, liver and bone. According to the great number of metastases, treatment of the breast cancer disease is difficult. Thus, the major cause of death for breast cancer patients is related to their individual systemic disease [Disibio and French, 2008]. 


\subsubsection{Therapy}

Therapeutic options for breast cancer treatment cover surgery, radiotherapy and systemic treatments. Surgeries with breast-conserving strategies are a common treatment for cancers having a restricted size. Large primary tumors are often reduced in size by primary chemotherapy before removal. The surgery is often followed by a postoperative radiotherapy. Systemic treatments are differentiated in cytotoxic chemotherapy consisting of mainly anthracyclines and taxanes, endocrine therapy with aromatase inhibitors, tamoxifen as competitive ER-antagonist and $\mathrm{GnRH}$-analogs, and therapies against molecular targets as HER2 with trastuzumab as antibody against the receptor [Veronesi et al., 2005, DKG. Deutsche Krebsgesellschaft. DGGG. Deutsche Gesellschaft für Gynäkologische und Geburtshilfe, 2012]. The kind of therapy is dependent on the intrinsic subtype (see chapter 1.1.2). ER and/or PR positive tumors are treated primarily with an endocrine therapy, optionally supported by a cytotoxic regime. ER and/or PR negative tumors get a cytotoxic therapy. All of the HER2 positive tumors are treated with an anti-HER2 therapy [Goldhirsch et al., 2011].

During therapy of metastatic breast cancer, additional treatment of bone metastases can be carried out by surgery, radiotherapy or therapy with bisphosphonates and receptor activator of nuclear factor kappa-B ligand (RANKL)-inhibitors [DKG. Deutsche Krebsgesellschaft. DGGG. Deutsche Gesellschaft für Gynäkologische und Geburtshilfe, 2012].

\subsection{Metastasis}

Cancer diseases are often characterized by primary tumors and secondary tumor sites formed by metastasizing tumor cells. Mechanisms, which allow tumor cells to arise from a solid tumor, disseminate and colonize distant organs, are summarized as metastatic processes. The earliest hypothesis on metastasis, the "seed and soil" hypothesis, was proposed by Paget in 1889 describing a cross-talk between selected tumor cells, the "seed", and specific organ microenvironments, the "soil". Metastatic processes require both, the intrinsic properties of the tumor cells and the response of the host environment for efficient metastasis [Paget, 1889]. Later studies improved this hypothesis by differentiating three principles: tumor cells and host cells are both forming primary tumors and metastases, metastasis includes different mechanisms all necessary for building metastases and metastasis is depending on the organ microenvironment [Fidler, 2003].

The focus of the following information is based on the distant metastasis processes. Regional metastasis commonly depends on mechanical and anatomical conditions. 


\subsubsection{Metastasis cascade}

Metastasis is a multi-step process. First, tumor cells have to break through the cell structure of the solid primary tumor before they can invade the extracellular matrix (ECM) and connecting cell layers. The metastatic cells intravasate into blood and lymphatic vessels. There, they are transported through the vasculature. Before they extravasate into distant organ tissue, the cells adhere to the vessels. The metastatic cells have to adapt to the microenvironment of the local tissue. Then, they proliferate and form micrometastases. By developing a vascular network, the metastases grow and are clinically detectable after time [Fidler, 2003, Valastyan and Weinberg, 2011]. This process is illustrated in figure 1.1.

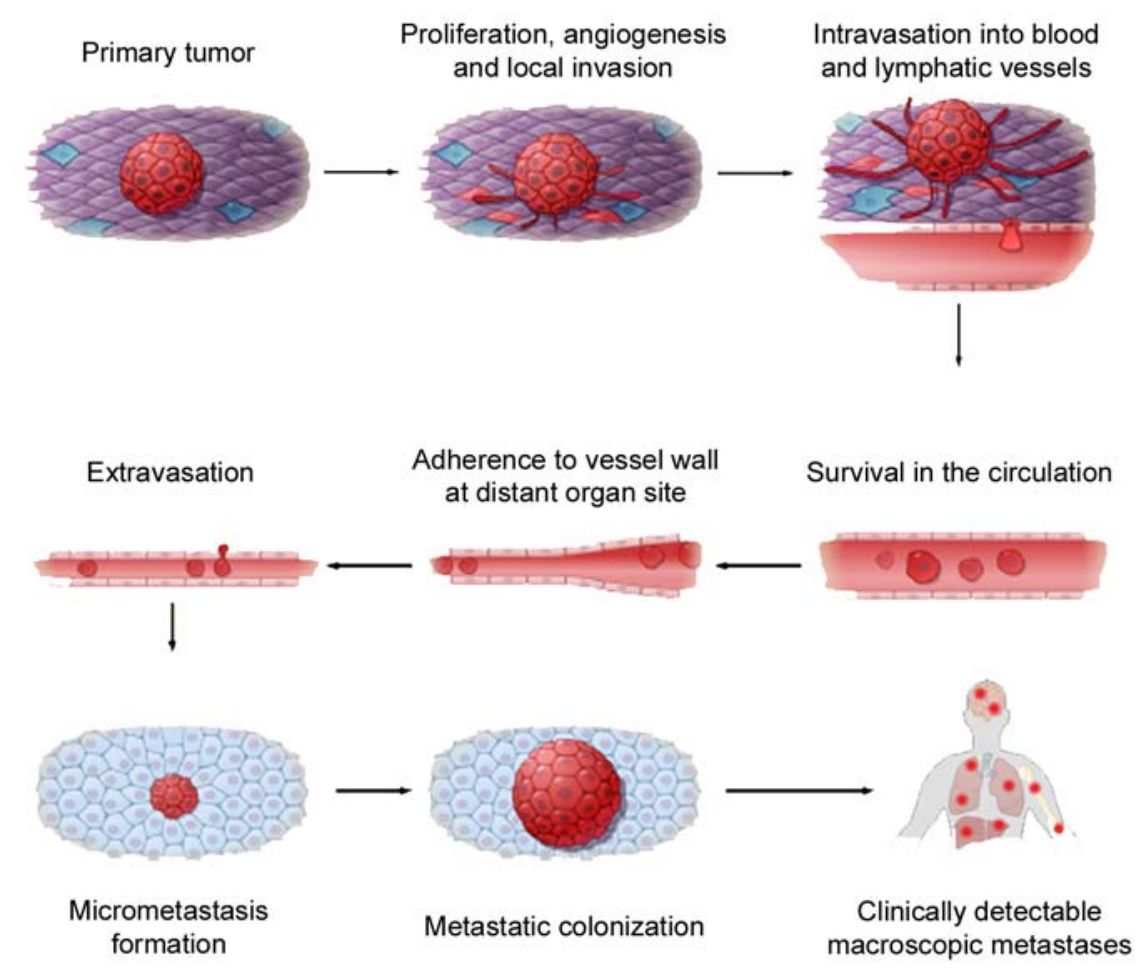

Figure 1.1: Metastasis cascade

The invasion-metastasis cascade describes the exit of tumor cells from the primary site by local invasion and intravasation, the systemic translocation requiring survival in the circulation, adaption to distant tissues by extravasation and metastases formation [Valastyan and Weinberg, 2011], modified.

The metastasis cascade covers some very complex and insufficient processes. Local invasion of single tumor cells requires the ability to defy the barriers of normal epithelium of the surrounding tissue. Different kinds of cellular invasion are possible (see chapter 1.2.3). Intravasation depends on the structural properties of the vessels. The tumor itself releases diverse growth factors to extend its vascular supply. This process is called neoangiogenesis. The formed vessels show differences compared to physiological vasculature. They are more permeable and leaky and allow an easier intravasation. When the cells reach the circulation, they have to survive the transport. 
Normally, the adhesion to the ECM is essential for epithelial cells and loss of this interaction leads physiologically to apoptosis. In addition, the circulating tumor cells (CTCs) are exposed to hemodynamic shear forces and cells of the immune system. The CTCs have to overcome these conditions, for example by passing the blood stream only for a short time or by masking themselves by interaction with blood platelets in order to arrive at distant organs. The location of settlement of the CTCs is not arbitrary. Arrest at distant sites can be influenced by physical conditions as limited diameters of the vessels or by interaction of liberated molecules of the target tissue with the CTCs and directed "homing" (see chapter 1.2.4). Extravasation of the disseminated tumor cells (DTCs) is characterized by the properties of the cells and the site of metastasis. Tissue as bone or liver are more permeable for penetrating, whereas extravasation into the brain requires overcoming the blood-brain barrier. Thus, the tumor cells need different mechanisms to enter distant organs. After extravasation, the tumor cells have to adapt to the foreign microenvironment to survive and form micrometastases. The microenvironment of the distant organ itself can be changed by factors released by the primary tumor to optimize the settlement of the DTCs. For further proliferation and colonization of the metastases, the target tissue and the tissue of the primary tumor are important. Macrometastases can only develop, if the interaction of host and occupying cells facilitates the active growth of metastases. Most of the DTCs remain dormant by either attrition or a continuous proliferation which does not exceed the apoptotic rate of the micrometastases [Valastyan and Weinberg, 2011].

The metastasis cascade is are very inefficient process and it needs continuous changes of physiological cellular behavior to generate clinically detectable macrometastases.

\subsubsection{Progression of metastasis}

The dissemination process of tumor cells leading to a systemic disease is mainly described by two models: the linear progression model and the parallel progression model. The major difference between these models is the temporal development and the beginning of metastasis and dissemination.

The linear progression model describes a late dissemination of tumor cells at distant organs. Development of aggressive tumor cells takes place within the primary tumor of advanced cancers by mutation and selection processes. When these tumor cell clones reach an autonomous growth as a small fraction of fully malignant cells, they are able to leave the primary tumor and colonize at distant sites [Hunter et al., 2008]. Evidence for this model is given by a correlation between tumor size and frequency of metastasis as basis of the TNM classification system (see chapter 1.1.2). The death rates of patients with $\mathrm{T} 2$ tumors is higher than of patients with $\mathrm{T} 1$ tumors [Klein, 2009].

The parallel progression model defines an early dissemination of tumor cells. Premalignant cells 
disseminate to distant sites before and during the primary tumor develops. All of the cells expand genetic and epigenetic changes independently and in parallel. They show discrepancies in their gene expression profiles. Diagnosis of metastases in early stage cancers and cancer diseases of unknown primary sites are evident for this progression model [Pantel and Brakenhoff, 2004, Klein, 2009].

Progression of metastasis shows various clinical events which can not be described by one single model. The individual aspects of metastasis can be explained by either the linear or the parallel progression model but not for cancer metastasis in general.

\subsubsection{Motility mechanisms of cells}

One initial step of the metastasis cascade (see chapter 1.2.1) is dissemination of tumor cells. Therefore, changes of the cellular plasticity have to be performed to gain motile cells which can invade into the circulating system. Different kinds of motility mechanisms are known: migration of single cells via mesenchymal-like or amoeboid-like migration and collective cell migration. The choice of migration mode is depending on present cell-cell and cell-matrix adhesions, ability of ECM remodeling and the constitution of the surrounding ECM.

Mesenchymal and amoeboid migration of individual cells is characterized by a loss of cell-cell adhesions. Mesenchymal-like cells have a polarized cell structure and a spindle-shaped and fibroblastlike morphology. They are able to form cell-matrix interactions by integrins and cytoskeletal protrusions. These cells move by pulling of an ECM substrate via generation of traction forces. Thereby, they interact with focal adhesions in the front of the cell and contractions of the retraction fibres in the cell tail. Invasion through the ECM is afforded by proteolysis with matrix metalloproteinases (MMPs) (see figure 1.2). In contrast, cell migration in an amoeboid-like manner lacks focal adhesions and stress fibres. The rounded cells move without cell-matrix adhesions by cytoskeletal contractions in a "push-and-squeeze" type. Cell propulsions allow a forward pushing of the cell body through gaps in the ECM without its proteolytic degradation (see figure 1.2). Collective migration is characterized by migrating cell clusters with active cell-cell adhesions. These clusters move by membrane protrusions, integrin-mediated focal adhesions and contractions via the actin-myosin apparatus. The cells use pulling forces on their neighboring cells and keep connection by adherent junctions (see figure 1.2). The collectively migrating cells degrade the ECM for invasion by proteolysis. All kinds of motility mechanisms are described for tumor

cells. Single-cell migration is important for metastasis to distant organs whereas collective cell migration is observed during cancer progression by local invasion [Friedl and Wolf, 2010, Yilmaz and Christofori, 2010, Parri and Chiarugi, 2010, van Zijl et al., 2011].

The change from one motility mechanism to another is reversible and dependent on the structure of the ECM, the availability of proteases for remodeling the surrounding tissue and the existence 
of cell-cell adhesion. These factors can alter during cell movement. In figure 1.2, the different conversions are graphed. Collectively migrating cells can change their motility mode to individual cell migration by epithelial to mesenchymal transition (EMT) to become mesenchymal-like cells or by collective to amoeboid transition (CAT) to become amoeboid-like cells. The reverse processes are named MET for mesenchymal to epithelial transition respectively amoeboid to collective transition (ACT). The conversion of mesenchymal-like cells to amoeboid-like cells is described as mesenchymal to amoeboid transition (MAT) respectively AMT for amoeboid to mesenchymal transition for the reverse process.

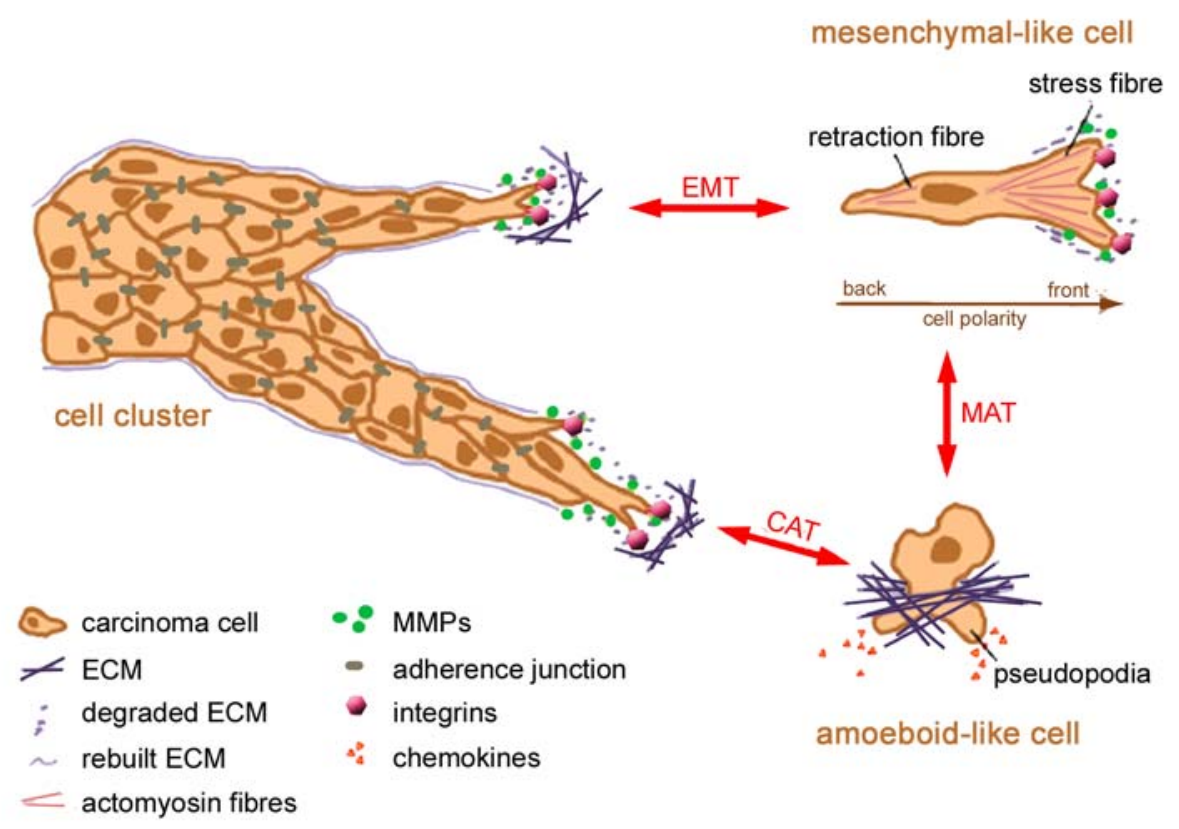

Figure 1.2: Mechanisms of cell invasion

Plasticity of invading cells is illustrated for cell clusters, mesenchymal-like and amoeboid-like cells. The conversions from one mode to another are reversible. EMT, epithelial-mesenchymal-transition; MAT, mesenchymal-amoeboidtransition; CAT, collective-amoeboid-transition; ECM, extracellular matrix; MMPs, matrix metalloproteinases [van Zijl et al., 2011], modified.

EMT is characterized by a loss of epithelial markers important for cell-cell adhesions as epithelial cadherin ( $\mathrm{CDH} 1)$ and an increase in mesenchymal markers as neuronal cadherin ( $\mathrm{CDH} 2)$, the so-called "cadherin-switch", and vimentin (VIM), an intermediate filament. EMT occurs physiologically during implantation, embryogenesis and organ development (type 1 EMT). In addition, EMT is associated with tissue regeneration and organ fibrosis (type 2 EMT). Evidence for a type 3 EMT involved in cancer progression and metastasis is given [Kalluri and Weinberg, 2009].

During MAT and CAT, mesenchymal respectively collective migrating cells become amoeboid-like. These cells show less cell-ECM interactions and invade by squeezing through gaps in the ECM without use of proteases and ECM degradation. CAT is characterized by a loss CDH1-mediated adhesions and develops mainly after integrin inhibition [van Zijl et al., 2011]. MAT is often 
induced by reduced Ras-related C3 botulinum toxin substrate (Rac) activity and an increased Ras homolog gene family - Rho-associated protein kinase (Rho/ROCK) signaling. The Rac pathway is responsible for extensions of the leading edge of the cells, for focal adhesions by integrins as basis for mesenchymal-like migration and for an elongated cell morphology. The Rho/ROCK pathway controls actomyosin retractions of the trailing cell edge leading to a nonadhesive amoeboid-like migration and a rounded cell morphology. These two pathway are counterbalancing each other [Friedl and Wolf, 2010, Parri and Chiarugi, 2010, van Zijl et al., 2011].

The kind of cell motility differs depending on the molecular genetics of the tumor cells and the constitution of the surrounding tissue. Thus, changes of the motility mode happen constantly and reversibly.

\subsubsection{Microenvironmental dependency of metastatic settlement}

With regard to Paget's "seed and soil" hypothesis and clinical observations, evidence is given for a dependency of metastasis to specific organs by certain tumor types. Typical sites of metastases are lung, liver, bone and brain. Some sites of metastatic settlement can be explained by physical and physiological forces as mechanical lodgement of CTCs and high vascularization rates characteristic for liver. But in contrast, the spleen is rarely affected by metastases, while it is also highly vascularized. Additionally, there are millions of tumor cells circulating the blood and less than $0.01 \%$ of the CTCs are successfully forming metastases [Paget, 1889, Fidler, 2003, Mathot and Stenninger, 2012]. Beside these "passive" criteria of metastatic formation, molecular processes as homing in preferential sites and a favorable environment for growth are required for an "active" and selective metastasis. The distant organ site itself and the primary tumor are considered as activators of the conditioning of the so-called "premetastatic/metastatic niche". On the one hand, proinflammatory proteins and chemotactic factors are released of the host organ for promoting the attachment and homing of the CTCs. On the other hand, the primary tumor changes the host microenvironment by signaling factors and CTCs. The CTCs, which were previously arrested at the distant site, can modify the microenvironment for a favorable establishment of metastases by later CTCs [Bidard et al., 2008].

There are some homing mechanisms respectively factors known for metastasizing tumor cells to lung, liver, bone and brain. In lung metastasis, organ specific upregulation of vascular cell adhesion protein 1 (VCAM-1) is described to support metastases growth. Upregulation of cyclooxygenase 2 (COX-2) in the liver leads to a resistance of the immune response of the CTCs and allows a better settlement for liver metastases. An important mechanism in bone metastasis is the chemokine (C-X-C motif) receptor 4/chemokine (C-X-C motif) ligand 12 (CXCR4/CXCL12) interaction. It is characterized by a receptor expression in the tumor cells and a release of CXCL12 at target organs leading to a directed metastasis. Furthermore, the tumor cells are able to release parathy- 
roid hormone-related protein (PTHrP), which interacts with the bone formation and resorption processes, leading to better angiogenesis and immune suppression via transforming growth factor $\beta$ (TGF $\beta$ ). In metastasis of the brain, the vascular endothelial growth factor (VEGF) seems to be involved in angiogenesis and growth [Mathot and Stenninger, 2012].

\subsection{KISS1}

The KISS1 gene and its peptides are involved in physiological and pathophysiological mechanisms as neuroendocrine regulation of reproduction, pregnancy and tumor metastasis. The focus of the following work lies in the antimetastatic properties of the gene.

\subsubsection{KISS1 and its receptor GPR54}

The KISS1 gene was discovered as metastasis suppressor gene. The antimetastatic effect was first identified in vivo. Mice showed less metastases after injection of melanoma and breast cancer cell lines, if cells were transfected with KISS1 [Welch et al., 1994, Lee et al., 1996, Miele et al., 1996, Lee and Welch, 1997a,b]. The KISS1 gene encodes a peptide of 145 amino acids, which is cleaved proteolytically in shorter peptides, the kisspeptins (KP). KP-54, KP-14, KP-13 and KP-10 are characterized by their common amidated $\mathrm{C}$-terminal. The kisspeptins are agonists binding to the KISS1 receptor GPR54. KP-10 shows the highest potency upon the kisspeptins in receptor activation [Kotani et al., 2001, Muir et al., 2001, Ohtaki et al., 2001].

GPR54 is a $G_{q / 11}$-protein coupled receptor responsible for phospholipase $C$ (PLC) activation and phosphatidylinositol 4,5-bisphosphate $\left(\mathrm{PIP}_{2}\right)$ hydrolysis, $\mathrm{Ca}^{2+}$ mobilization, arachidonic acid release, extracellular signal regulated kinases 1 and 2 (ERK1/2) and p38 mitogen activated protein (MAP) kinase phosphorylation and stress fibre formation [Kotani et al., 2001, Stafford et al., 2002, Navenot et al., 2005]. The receptor is involved in cell cycle arrest and apoptosis [Becker et al., 2005]. Its signaling is regulated by GPR serine/threonine kinase 2 (GRK2) and $\beta$-arrestin [Pampillo et al., 2009, Szereszewski et al., 2010].

The role of KISS1 and GPR54 expression in cancer tissue is not yet clear. In healthy tissue, KISS1 is expressed predominantly in the placenta, testis, pancreas and liver, whereas the receptor is detected mainly in brain, pancreas and placenta [Muir et al., 2001, Ohtaki et al., 2001]. In clinical samples, loss of KISS1 and GPR54 expression correlated with lymph node metastasis in esophageal squamous carcinoma [lkeguchi et al., 2004]. In ovarian carcinomas, KISS1 and GPR54 expression was associated with a good overall survival [Prentice et al., 2007]. Related results were observed in patients with pancreatic cancer [Nagai et al., 2009] and endometrial cancer [Kang et al., 2011]. Reduced KISS1 mRNA was detected in breast cancer tissue compared to normal 
tissue [Mooez et al., 2011]. In contrast, upregulated levels of KISS1 and GPR54 expression led to shorter relapse free survival in breast cancer patients [Martin et al., 2005, Marot et al., 2007]. High GPR54 expression was also found in breast, ovarian, small intestine and colon cancer compared to normal tissue [Ohtaki et al., 2001] and overexpression of KISS1 and GPR54 correlated with hepatocellular carcinoma progression [lkeguchi et al., 2003]. In summary, there is conflicting data comparing the expression of KISS1 and GPR54 with cancer progression.

\subsubsection{Cell motility and metastasis affected by kisspeptins}

Several studies in literature show an involvement of the KISS1/GPR54 system in cell motility mechanisms. KISS1 transfection led to reduced invasion and migration in ovarian and gastric carcinoma cells [Jiang et al., 2005, Li et al., 2012]. Treatment with KP-10 showed similar effects in trophoblasts and cancer cell lines as renal, pancreatic, breast and endometrial carcinoma cells [Bilban et al., 2004, Masui et al., 2004, Shoji et al., 2009, Olbrich et al., 2010b, Chen et al., 2011, Kang et al., 2011, Roseweir et al., 2012]. Additionally, invasion of cells transfected to overexpress GPR54 was also inhibited by KP-10 [Hori et al., 2001].

The KISS1/GPR54 system seems to be participating in chemokinetic and chemotactic processes. The activation of GPR54 by KP-10 affects the Rho-ROCK axis leading to changes in the actin cytoskeleton and alterations of the cell shape [Takino et al., 2003, Navenot et al., 2009, Shoji et al., 2009]. Evidence is given for a regulation of focal adhesions by an ERK $1 / 2$ - glycogen synthase kinase $3 \beta$ (GSK3 $\beta$ ) - focal adhesion kinase (FAK) feedback loop via EGFR transactivation in trophoblasts by KP-10 and GPR54 [Roseweir et al., 2012]. Chemotactic responses of the CXCR4/CXCL12 system are inhibited by KP-10 treatment in cells transfected with GPR54 and in breast cancer cells with an endogenous GPR54 expression [Navenot et al., 2005, Olbrich et al., 2010b]. Another aspect for the reduced invasion by the KISS1/GPR54 system is a negative regulation of MMP expression [Yan et al., 2001, Yoshioka et al., 2008, Li et al., 2012] (see chapter 1.2.3).

The first experiments on KISS1 identified the gene as metastasis suppressor in xenografts with melanoma cells transfected to overexpress KISS1 [Lee et al., 1996, Lee and Welch, 1997a]. Continuing studies verified the effect in in vivo experiments with KISS1 transfected breast, ovarian and pancreatic cancer cells [Lee and Welch, 1997b, Jiang et al., 2005, McNally et al., 2010]. Peripherally administered KP-10 also reduced metastasis in xenografts with melanoma, prostate and endometrial cancer cells [Ohtaki et al., 2001, Cho et al., 2009b, Kang et al., 2011]. 


\subsubsection{Kisspeptin analogs}

The kisspeptins are related to the RF-amide family. These peptides share a common Arg-Pheamide C-terminal and prolactin-releasing peptide and neuropeptide FF belong to this group. The most potent kisspeptin is KP-10. Its amino acid structure is Tyr-Asn-Trp-Asn-Ser-Phe-Gly-LeuArg-Phe- $\mathrm{NH}_{2}$. The C-terminal Arg-Phe is critical for its biologic activity at GPR54 [Clements et al., 2001, Kotani et al., 2001, Orsini et al., 2007]. Several kisspeptin analogs were developed. One aim was to enhance the low stability of the kisspeptins and another, to increase the activation potency or antagonize their effects.

Kisspeptins have a very short lifetime especially in serum [Tomita et al., 2008]. This is due to a rapid degradation by MMPs, which inactivate the peptides by cleavage of the Gly-Leu peptide bond in the C-terminal region [Takino et al., 2003]. Gly-Leu isosteres were developed showing a resistance against MMP mediated digestion and a prolonged half-life [Tomita et al., 2008]. Enantiomer changes from L-amino acids to D-amino acids are another possibility to stabilize secondary structures [Curtis et al., 2009].

Receptor activation is mainly based on the five amino acids at the C-terminal [Niida et al., 2006, Gutierrez-Pascual et al., 2009, Roseweir et al., 2009]. A higher potency is reached by replacement of Phe at position ten by Trp. This peptide is known as the murine KP-10 [Clements et al., 2001]. The aromatic group at position six seems to be important for agonistic activity [Tomita et al., 2007, 2006]. A correlation between membrane binding and agonist activity of the peptides was shown [Lee et al., 2009]. Experiments on different structures than analogs of kisspeptin revealed agonistic and antagonistic substances with an peptidic form and also small molecules [Kobayashi et al., 2010, Kuohung et al., 2010].

\subsection{Outline of the thesis}

One aim of this project was to characterize human breast cancer cell lines according to their behavior in coculture. The experimental setting was established in the laboratory by a former member. Breast cancer cells were cocultivated with osteoblast-like osteosarcoma cells to study a possible interaction of both cell kinds. Coculture conditions led to changes of breast cancer cell invasion properties [von Alten et al., 2006]. In continuing experiments, this effect should be further investigated. Therefore, different breast cancer cell lines were studied in monoculture and coculture for their invasion and migration. Cells were classified morphologically and on molecular levels to identify a possible cell motility mechanisms. The main focus was set upon EMT and additional experiments were done with TGF $\beta_{1}$, which is known as important activator of EMT processes. 
In earlier works, it was shown, that the increased invasion during cocultivation was inhibited by kisspeptin-10, a peptide derived from the metastasis suppressor gene KISS1 [Olbrich et al., 2010b, Olbrich, 2010a]. The coculture system was developed originally as an in vitro model to mimic a bone metastasis microenvironment based on the preferred metastasis to bone in breast cancer patients. During this project, it should be checked, if a comparable effect of kisspeptin-10 could be detected in in vivo experiments. Studies in literature investigated the antimetastatic effect mainly in xenografts with cancer cells transfected with the KISS1 gene (see chapter 1.3.2). A peripheral administration of kisspeptin was only investigated for melanoma [Ohtaki et al., 2001], prostate [Cho et al., 2009b] and endometrial cancer cells [Kang et al., 2011] and up to date not for a breast cancer xenograft. Therefore, different breast cancer cell lines were used in mouse xenografts. Spontaneous metastasis was studied under treatment with kisspeptin-10 after intracardiac respectively orthotopic tumor cell injection. An effect of kisspeptin-10 on tumor growth was not expected, but observed during the experiments. Thus, further studies on proliferation in vitro were carried out to verify an antiproliferative effect of KP-10.

KISS1 and its peptides are an interesting aim of research and the kisspeptins may be a potent tool in treatment of metastatic diseases especially in patients suffering from breast cancer. Further studies on kisspeptin are required with regard to more potent and more stable analogs. Therefore, first experiments with a kisspeptin-10 analog were done in vitro under coculture conditions. 


\section{Material and methods}

\subsection{Material}

\subsubsection{Biological substances, chemicals, commercial reagents and kits}

The used biological substances, chemicals, commercial reagents and kits are shown in the following tables.

\begin{tabular}{|l|l|}
\hline Biological substances & Manufacturer \\
\hline \hline Bovine serum albumin (BSA) & Sigma, Steinheim, Germany \\
\hline $\begin{array}{l}\text { DK6-Kisspeptin-10; peptide } \\
\text { Tyr-Asn-Trp-Asn-Ser-dLys-Gly-Leu-Arg-Phe }\end{array}$ & PSL, Heidelberg, Germany \\
\hline Fetal calf serum (FCS) & Biochrom, Berlin, Germany \\
\hline Fetal calf serum (FCS), charcoal treated & PAN Biotech, Aidenbach, Germany \\
\hline Instant skimmed milk powder, spray-dried & Saliter, Obergünzburg, Germany \\
\hline Insulin, Insuman ${ }^{\circledR}$ rapid ${ }^{\circledR}$ & Sanofi-Aventis, Frankfurt, Germany \\
\hline $\begin{array}{l}\text { Kisspeptin-10; peptide } \\
\text { Tyr-Asn-Trp-Asn-Ser-Phe-Gly-Leu-Arg-Phe }\end{array}$ & PSL, Heidelberg, Germany \\
\hline L-Glutamine & Biochrom, Berlin, Germany \\
\hline Matrigel ${ }^{T M}$ Basement Membrane Matrix & BD Biosciences, Bedford, MA, USA \\
\hline Pen Strep, Gibco ${ }^{\circledR}$ & Life Technologies, Darmstadt, Germany \\
\hline TGF $\beta 1$ & Sigma-Aldrich, Saint Louis, MO, USA \\
\hline Trypsin-EDTA $(1 x)$ & PAA, Pasching, Austria \\
\hline
\end{tabular}

Table 2.1: Biological substances 


\begin{tabular}{|l|l|}
\hline Chemicals & Manufacturer \\
\hline \hline Agarose, peqGOLD universal & Peqlab, Erlangen, Germany \\
\hline 6-Aminohexanoic acid & Roth, Karlsruhe, Germany \\
\hline Ammonium persulfate (APS) & AppliChem, Darmstadt, Germany \\
\hline Boric acid & Roth, Karlsruhe, Germany \\
\hline Bio-Rad Protein Assay & BIO-RAD, München, Germany \\
\hline Chloroform & Merck, Darmstadt, Germany \\
\hline Dimethyl sulfoxide (DMSO) & Merck, Darmstadt, Germany \\
\hline Ethylenediaminetetraacetic acid (EDTA) & Sigma, Steinheim, Germany \\
\hline Ethanol (EtOH) & Merck, Darmstadt, Germany \\
\hline Ethidium bromide & Roth, Karlsruhe, Germany \\
\hline G418 sulfate & PAA, Pasching, Austria \\
\hline Glycine & USB, Cleveland, OH, USA \\
\hline Haematoxylin & Merck, Darmstadt, Germany \\
\hline holo-Transferrin human & Sigma, Steinheim, Germany \\
\hline Hydrochloric acid $(\mathrm{HCl}), 1 \mathrm{M}$ & Merck, Darmstadt, Germany \\
\hline Hydrogen peroxide $\left(\mathrm{H}_{2} \mathrm{O}_{2}\right)$ & Merck, Darmstadt, Germany \\
\hline Isopropyl alcohol & Th.Geyer, Renningen, Germany \\
\hline 2-Mercaptoethanol & Sigma, Steinheim, Germany \\
\hline Methanol (MeOH) & Th.Geyer, Renningen, Germany \\
\hline Sodium hydroxide $(\mathrm{NaOH}), 1 \mathrm{M}$ & Merck, Darmstadt, Germany \\
\hline Paraformaldehyde & Roth, Karlsruhe, Germany \\
\hline Potassium chloride $(\mathrm{KCl)}$ & Roth, Karlsruhe, Germany \\
\hline Sodium chloride $(\mathrm{NaCl)}$ & USB, Cleveland, OH, USA \\
\hline Sodium dodecyl sulfate (SDS) & Merck-Schuchardt, Hohenbrunn, Germany \\
\hline Tetramethylethylenediamine (TEMED) & BIO-RAD, München, Germany \\
\hline Tris & USB, Cleveland, OH, USA \\
\hline Tween & Sigma, Steinheim, Germany \\
\hline & \\
\hline
\end{tabular}

Table 2.2: Chemicals 


\begin{tabular}{|l|l|}
\hline Reagent or kit & Manufacturer \\
\hline \hline AEC substrate chromogen & Dako, Carpinteria, CA, USA \\
\hline alamarBlue ${ }^{\circledR}$ & AbD Serotec, Oxford, UK \\
\hline Aquatex ${ }^{\circledR}$ & Merck, Darmstadt, Germany \\
\hline CelLytic $^{\text {TM }}$ buffer & Sigma, St. Lois, MO, USA \\
\hline Chromatography paper 3MM Chr & Whatman, GE Healthcare, Freiburg, Germany \\
\hline DNA Ladder, 100 bp & Life Technologies, Darmstadt, Germany \\
\hline dNTPs & Roche Diagnostics, Mannheim, Germany \\
\hline ECL Immobilon ${ }^{\circledR}$ Western & Millipore, Schwalbach \\
\hline Histostain ${ }^{\circledR}$ Bulk Kit & Life Technologies, Darmstadt, Germany \\
\hline KAPA2GTM Fast, $2 x$ ReadyMix with Dye & Peqlab, Erlangen, Germany \\
\hline NucleoSpin ${ }^{\circledR}$ Tissue Kit and Filters & Macherey-Nagel, Düren, Germany \\
\hline NuPAGE ${ }^{\circledR} 4 x$ LDS sample buffer & Life Technologies, Darmstadt, Germany \\
\hline NuPAGE ${ }^{\circledR} 10 x$ sample reducing agent & Life Technologies, Darmstadt, Germany \\
\hline p(dT) 15 primer & Roche Diagnostics, Mannheim, Germany \\
\hline ProSieve ${ }^{\circledR}$ 50 Gel solution & Lonza, Rockland, ME, USA \\
\hline Protease Inhibitor & Sigma, St. Lois, MO, USA \\
\hline Protein Assay Reagent & BIO-RAD, München, Germany \\
\hline Protein-Marker I and V & Peqlab, Erlangen, Germany \\
\hline PVDF membranes & Millipore, Billerica, MA, USA \\
\hline RNase inhibitor, RNasin ${ }^{\circledR}$ & Promega, Madison, WI, USA \\
\hline RNeasy ${ }^{\circledR}$ Mini Kit & Qiagen, Hilden, Germany \\
\hline SensiFAST SYBR No-ROX One-Step Kit & Bioline, Luckenwalde, Germany \\
\hline $18 S$ rRNA Control Kit & Eurogentec, Köln, Germany \\
\hline TaqMan ${ }^{\circledR}$ Universal PCR Master Mix & Life Technologies, Darmstadt, Germany \\
\hline TriFast ${ }^{\text {TM }}$, peqGOLD & Peqlab, Erlangen, Germany \\
\hline
\end{tabular}

Table 2.3: Commercial reagents and kits 


\subsubsection{Buffers, solutions and media}

Commonly used buffers, solutions and media are shown in the following tables.

\begin{tabular}{|c|c|}
\hline Liquid & Receipt or manufacturer \\
\hline Agarose $(1.5 \% ; 2 \%)$ & $1.50 \% / 2 \%(w / v)$ Agarose in $\frac{1}{2} \times$ TBE \\
\hline Ammonium persulfate (APS) $(10 \%)$ & $10 \%(\mathrm{w} / \mathrm{v})$ APS in $\mathrm{H}_{2} \mathrm{O}$ \\
\hline Anode buffer & $30 \mathrm{mM}$ Tris, $20 \% \mathrm{MeOH}$ in $\mathrm{H}_{2} \mathrm{O}$; pH 10.4 \\
\hline Anode buffer (conc.) & $300 \mathrm{mM}$ Tris, $20 \% \mathrm{MeOH}$ in $\mathrm{H}_{2} \mathrm{O} ; \mathrm{pH} 10.4$ \\
\hline Blocking solution & $5 \%(\mathrm{w} / \mathrm{v})$ instant skimmed milk powder in TBST \\
\hline Cathode buffer & $\begin{array}{l}40 \mathrm{mM} \text { 6-aminohexanoic acid, } 25 \mathrm{mM} \text { Tris, } 20 \% \mathrm{MeOH} \text { in } \\
\mathrm{H}_{2} \mathrm{O} ; \mathrm{pH} 9.4\end{array}$ \\
\hline Ethanol $(70 \%)$ & $70 \%(v / v) \mathrm{EtOH}$ in $\mathrm{H}_{2} \mathrm{O}$ \\
\hline Ethidium bromide staining bath & $1,72 \mu \mathrm{M}$ ethidium bromide in $\mathrm{H}_{2} \mathrm{O}$ \\
\hline Hydrogen peroxide $(3 \%)$ & $3 \%(\mathrm{v} / \mathrm{v}) \mathrm{H}_{2} \mathrm{O}_{2}$ in $\mathrm{H}_{2} \mathrm{O}$ \\
\hline Ketamine, Ketavet ${ }^{\circledR}$ & Bayer Animal Health GmbH, Leverkusen, Germany \\
\hline Kisspeptin-10 solutions & $\begin{array}{l}\text { KP-10 (initially dissolved in DMSO) was diluted in water for } \\
\text { injection to get the used concentrations for in vitro } \\
\text { experiments; KP-10 (initially dissolved in DMSO) was diluted } \\
\text { in PBS with HAc [10 mM] to get the solutions for in vivo } \\
\text { experiments }\end{array}$ \\
\hline Paraformaldehyde (4\%) & $4 \%(\mathrm{v} / \mathrm{v})$ paraformaldehyde in $\mathrm{H}_{2} \mathrm{O}$ \\
\hline Phosphate buffered saline (DPBS) & PAN Biotech, Aidenbach, Germany \\
\hline SDS electrophoresis buffer & $25 \mathrm{mM}$ Tris, $192 \mathrm{mM}$ Glycin, $0.1 \%(\mathrm{w} / \mathrm{v}) \mathrm{SDS}$ in $\mathrm{H}_{2} \mathrm{O}$ \\
\hline Sodium chloride $(\mathrm{NaCl}), 0.9 \%$ & B. Braun Melsungen AG, Melsungen, Germany \\
\hline Sol B & 1.5 M Tris in $\mathrm{H}_{2} \mathrm{O} ; \mathrm{pH} 8,8(\mathrm{HCl})$ \\
\hline Sol C & $10 \%(w / v) S D S$ in $\mathrm{H}_{2} \mathrm{O}$ \\
\hline Sol D & 0,5 M Tris in $\mathrm{H}_{2} \mathrm{O}$; pH 6,8 $(\mathrm{HCl})$ \\
\hline TBE & $8.4 \mathrm{mM}$ Tris, $90 \mathrm{mM}$ boric acid, $2.8 \mathrm{mM}$ EDTA in $\mathrm{H}_{2} \mathrm{O}$ \\
\hline TBS & $137 \mathrm{mM} \mathrm{NaCl}, 2.7 \mathrm{mM} \mathrm{KCl}, 24.8 \mathrm{mM}$ Tris in $\mathrm{H}_{2} \mathrm{O}$; $\mathrm{pH} 7.4$ \\
\hline TBST & $0.1 \%$ Tween in TBS \\
\hline Transfer buffer & $25 \mathrm{mM}$ Tris, $192 \mathrm{mM}$ Glycin, $20 \%$ (v/v) $\mathrm{MeOH}$ in $\mathrm{H}_{2} \mathrm{O}$ \\
\hline Xylazine, Rompun ${ }^{\circledR}$ & Bayer Animal Health GmbH, Leverkusen, Germany \\
\hline
\end{tabular}

Table 2.4: Buffers and solutions 


\begin{tabular}{|c|c|}
\hline Medium & Receipt and manufacturers \\
\hline $\begin{array}{l}\text { Cell culture medium } \\
\text { for breast cancer } \\
\text { cell lines }\end{array}$ & $\begin{array}{l}\text { MEM with stable glutamine, phenol red (Biochrom, Berlin, Germany) } \\
10 \%(\mathrm{v} / \mathrm{v}) \mathrm{FCS} \\
100 \mathrm{U} / \mathrm{ml} \text { penicillin and } 100 \mu \mathrm{g} / \mathrm{ml} \text { streptomycin } \\
0.05 \mathrm{IU} / \mathrm{ml} \text { insulin } \\
1 \mu \mathrm{g} / \mathrm{ml} \text { transferrin }\end{array}$ \\
\hline $\begin{array}{l}\text { Cell culture medium } \\
\text { for osteosarcoma } \\
\text { cell line }\end{array}$ & $\begin{array}{l}\text { DMEM with stable glutamine, phenol red }\left(\text { Gibco }^{\circledR} \text {, Life Technologies, }\right. \\
\text { Darmstadt, Germany) } \\
10 \%(\mathrm{v} / \mathrm{v}) \mathrm{FCS} \\
100 \mathrm{U} / \mathrm{ml} \text { penicillin and } 100 \mu \mathrm{g} / \mathrm{ml} \text { streptomycin }\end{array}$ \\
\hline $\begin{array}{l}\text { Cell culture medium } \\
\text { for transfected B35 } \\
\text { cell clones }\end{array}$ & $\begin{array}{l}\text { DMEM with stable glutamine, phenol red }\left(\text { Gibco }^{\circledR} \text {, Life Technologies, }\right. \\
\text { Darmstadt, Germany) } \\
10 \%(\mathrm{v} / \mathrm{v}) \text { FCS } \\
1 \mathrm{mg} / \mathrm{ml} \mathrm{G418} \mathrm{sulfate}\end{array}$ \\
\hline $\begin{array}{l}\text { Experimental } \\
\text { medium } \\
\text { for breast cancer } \\
\text { and osteosarcoma } \\
\text { cell lines }\end{array}$ & $\begin{array}{l}\text { DMEM, without phenol red (Gibco }{ }^{\circledR}, \text { Life Technologies, Darmstadt, Germany) } \\
10 \%(\mathrm{v} / \mathrm{v}) \text { charcoal treated FCS } \\
100 \mathrm{U} / \mathrm{ml} \text { penicillin and } 100 \mu \mathrm{g} / \mathrm{ml} \text { streptomycin } \\
2 \mu \mathrm{mol} / \mathrm{ml} \text { L-glutamine }\end{array}$ \\
\hline $\begin{array}{l}\text { Experimental } \\
\text { medium } \\
\text { for transfected B35 } \\
\text { cell clones }\end{array}$ & $\begin{array}{l}\text { DMEM, without phenol red (Gibco }{ }^{\circledR} \text {, Life Technologies, Darmstadt, Germany) } \\
10 \%(\mathrm{v} / \mathrm{v}) \text { charcoal treated } \mathrm{FCS} \\
100 \mathrm{U} / \mathrm{ml} \text { penicillin and } 100 \mu \mathrm{g} / \mathrm{ml} \text { streptomycin } \\
2 \mu \mathrm{mol} / \mathrm{ml} \text { L-glutamine }\end{array}$ \\
\hline Freezing medium & Cell culture medium containing $5 \%$ (v/v) DMSO \\
\hline
\end{tabular}

Table 2.5: Media

\subsubsection{Enzymes}

\begin{tabular}{|l|l|}
\hline Enzyme & Manufacturer \\
\hline \hline DNase I recombinant, RNase-free & Roche Diagnostics, Mannheim, Germany \\
\hline RNase A & Qiagen, Hilden, Germany \\
\hline SuperScript ${ }^{T M}$ II Reverse Transcriptase & Life Technologies, Darmstadt, Germany \\
\hline Trypsin-EDTA $(0,05 \% / 0,02 \%$ in DPBS $)$ & PAA, Pasching, Austria \\
\hline
\end{tabular}

Table 2.6: Enzymes 


\subsubsection{Oligonucleotides}

\begin{tabular}{|c|c|c|c|}
\hline Gene & Sequence & $\begin{array}{l}\text { Product } \\
\text { length }\end{array}$ & Use \\
\hline ALU & $\begin{array}{l}\text { sense 5' CAT GGT GAA ACC CCG TCT CTA 3' } \\
\text { antisense 5' CC TCA GCC TCC CGA GTA 3' }\end{array}$ & - & In vivo studies, qPCR \\
\hline ALU probe & $5^{\prime}$ [YY]-ATT AGC CGG GCG TGG TGG CG-[BHQ-1] 3' & - & In vivo studies, qPCR \\
\hline $\mathrm{CDH} 1$ & $\begin{array}{l}\text { sense 5' ACA TTT CCC AAC TCC TCT CC 3' } \\
\text { antisense 5' CAG CCA TCC TGT TTC TCT TTC 3' }\end{array}$ & $222 \mathrm{bp}$ & $\begin{array}{l}\text { EMT marker, standard } \\
\text { PCR }\end{array}$ \\
\hline CXCL12 & $\begin{array}{l}\text { sense 5' CCG CGC TCT GCC TCA GCG ACG GGA 3' } \\
\text { antisense 5' CTT GTT TAA AGC TTT CTC CAG GTA 3' }\end{array}$ & $227 \mathrm{bp}$ & qPCR \\
\hline FN1 & $\begin{array}{l}\text { sense 5' TGA AGA GGG GCA CAT GCT GAA C 3' } \\
\text { antisense 5' AAT GCC ACG GCC ATA GCA GTA G 3' }\end{array}$ & $178 \mathrm{bp}$ & $\mathrm{qPCR}$ \\
\hline GPR54 & $\begin{array}{l}\text { sense 5' CGA CTT CAT GTG CAA GTT CGT C 3' } \\
\text { antisense 5' CAC ACT CAT GGC GGT CAG AG 3' }\end{array}$ & $82 \mathrm{bp}$ & $\begin{array}{l}\text { Breast cancer cell lines, } \\
\text { standard PCR }\end{array}$ \\
\hline GPR54 & $\begin{array}{l}\text { sense 5' TGA CCG CCA TGA GTG TGG AC 3' } \\
\text { antisense 5' GCG GAG TGG CTG TAG GAC AT 3' }\end{array}$ & $553 \mathrm{bp}$ & $\begin{array}{l}\text { GPR54 transfected cell } \\
\text { clones, standard PCR }\end{array}$ \\
\hline L7 & $\begin{array}{l}\text { sense 5' AGA TGT ACA GAA CTG AAA TTC 3' } \\
\text { antisense 5' ATT TAC CAA GAG ATC GAG CAA 3' }\end{array}$ & $357 \mathrm{bp}$ & $\begin{array}{l}\text { Housekeeping gene, } \\
\text { standard PCR }\end{array}$ \\
\hline MMP2 & $\begin{array}{l}\text { sense 5' CTC CTG ACA TTG ACC TTG GCA C 3' } \\
\text { antisense 5' TCA CAG TCC GCC AAA TGA ACC 3' }\end{array}$ & $150 \mathrm{bp}$ & qPCR \\
\hline PARP9 & $\begin{array}{l}\text { sense 5' CAG CAA CAA AAA ACC CAA GAC 3' } \\
\text { antisense 5' GAA AGG CAG CCA TAA GGA C 3' }\end{array}$ & $166 \mathrm{bp}$ & $\mathrm{qPCR}$ \\
\hline S100A4 & $\begin{array}{l}\text { sense 5' TCT CTC CTC AGC GCT TCT TC 3' } \\
\text { antisense 5' GCT GTC CAA GTT GCT CAT CA 3' }\end{array}$ & $\begin{array}{l}238 \mathrm{bp} / \\
239 \mathrm{bp}\end{array}$ & $\begin{array}{l}\text { EMT marker, standard } \\
\text { PCR }\end{array}$ \\
\hline SPARC & $\begin{array}{l}\text { sense 5' TGC TTC GGC ATC AAG CAG AAG G 3' } \\
\text { antisense 5' ACA TTG GGG GAA ACA CGA AGG G3' }\end{array}$ & $111 \mathrm{bp}$ & $\mathrm{qPCR}$ \\
\hline TACC1 & $\begin{array}{l}\text { sense 5' CTC AGC GAA TCA GAC AAG AC 3' } \\
\text { antisense 5' TCA TCT CCA AAA CTT CTT GCC 3' }\end{array}$ & $124 \mathrm{bp}$ & qPCR \\
\hline TCF21 & $\begin{array}{l}\text { sense 5' CCT TCT CCA GAC TCA AGA CCA C 3' } \\
\text { antisense 5' TGG TTC CAC ATA AGC GGC TC 3' }\end{array}$ & $237 \mathrm{bp}$ & qPCR \\
\hline TJP1 & $\begin{array}{l}\text { sense 5' TTC AAA AAC TCC CAC TTC TCC 3' } \\
\text { antisense 5' CCA TTG CTG TTA AAT ATG CCT C 3' }\end{array}$ & $240 \mathrm{bp}$ & $\begin{array}{l}\text { EMT marker, standard } \\
\text { PCR }\end{array}$ \\
\hline VIM & $\begin{array}{l}\text { sense 5' GCT GCT AAC TAC CAA GAC AC 3' } \\
\text { antisense 5' TCA GGT TCA GGG AGG AAA AG 3' }\end{array}$ & $208 \mathrm{bp}$ & $\begin{array}{l}\text { EMT marker, standard } \\
\text { PCR and } q P C R\end{array}$ \\
\hline YWHAZ & $\begin{array}{l}\text { sense 5' CTG GTG ATG ACA AGA AAG GG 3' } \\
\text { antisense 5' GAA CAC AGA GAA GTT AAG GGC 3' }\end{array}$ & $131 \mathrm{bp}$ & $\begin{array}{l}\text { Housekeeping gene, } \\
\text { qPCR }\end{array}$ \\
\hline
\end{tabular}

Table 2.7: Oligonucleotides 


\subsubsection{Antibodies}

\begin{tabular}{|l|l|l|}
\hline Description & Use & Manufacturer \\
\hline \hline ECL Anti-rabbit IgG, & Western blot analysis, & GE Healthcare, Freiburg, \\
horseradish peroxidase-linked & secondary antibody; dilution & Germany \\
species-specific whole antibody & $1: 33000$ & \\
\hline Monoclonal rabbit actin antibody & Western blot analysis; & Epitomics, Burlingame, CA, \\
& dilution 1:1000 & USA \\
\hline Polyclonal rabbit anti-GPR54 & Western blot analysis; & Alomone Labs, Jerusalem, \\
(AKR-001) & dilution 1:1000 & Israel \\
\hline $\begin{array}{l}\text { Polyclonal rabbit anti-human } \\
\text { GPR54 (SP4238P) }\end{array}$ & Immunocytochemical staining; & Acris, Herford, Germany \\
\hline
\end{tabular}

Table 2.8: Antibodies

\subsubsection{Biological material}

Cell lines were obtained from the American Type Culture Collection (ATCC; Manassas, VA, USA) and the German Collection of Microorganisms and Cell Cultures (DSMZ; Braunschweig, Germany).

\begin{tabular}{|l|l|l|l|}
\hline Cell line & Source & Histology & Reference \\
\hline \hline HCC 70 & Primary tumor & Primary ductal carcinoma & Gazdar et al. 1998 \\
\hline HCC 1806 & Primary tumor & $\begin{array}{l}\text { Primary acantholytic squamous cell } \\
\text { carcinoma }\end{array}$ & Gazdar et al. 1998 \\
\hline HCC 1937 & Primary tumor & Primary ductal carcinoma & Gazdar et al. 1998 \\
\hline MCF-7 & Pleural effusion & Adenocarcinoma & Soule et al. 1973 \\
\hline MDA-MB-231 & Pleural effusion & Adenocarcinoma & Cailleau et al. 1974 \\
\hline MDA-MB-435s & Pleural effusion & Ductal carcinoma & Cailleau et al. 1978 \\
\hline MDA-MB-453 & Pericardial effusion & Metastatic carcinoma & Cailleau et al. 1978 \\
\hline T47D & Pleural effusion & Ductal carcinoma & Keydar et al. 1979 \\
\hline ZR75-1 & Ascites & Ductal carcinoma & Engel et al. 1978 \\
\hline
\end{tabular}

Table 2.9: Human breast cancer cell lines

The cell line MDA-MB-435 was originally established as human breast cancer cell line. Over the last years several studies suggested a gene expression profile most compatible with a melanoma origin [Rae et al., 2004, Ross et al., 2000]. Others showed evidence for MDA-MB-435 original derived from breast cancer [Chambers, 2009, Sellappan et al., 2004]. According to this, the MDA-MB-435 cell line was used as breast cancer cell line.

\begin{tabular}{|l|l|l|l|}
\hline Cell line & Origin & Histology & Reference \\
\hline \hline MG63 & Bone & Osteosarcoma & Billiau et al. 1977 \\
\hline
\end{tabular}

Table 2.10: Osteosarcoma cell lines 
Transfected cell clones were kindly provided by Prof. Millar, Edinburgh, UK.

\begin{tabular}{|l|l|l|l|l|l|}
\hline Cell line & Origin & Histology & Reference & Transfection & Clones \\
\hline \hline B35 & Central nervous system & Neuroblastoma & Schubert et al. 1974 & mGPR54 & $1,2,3,4,5,6$ \\
\hline
\end{tabular}

Table 2.11: Transfected cell clones

\subsubsection{Animals}

Animals used in in vivo experiments were obtained from Charles River, Wilmington, MA, USA.

\begin{tabular}{|l|l|}
\hline Strain & Genotype \\
\hline \hline CD1 nude mouse & Crl:CD1-Foxn1 \\
\hline
\end{tabular}

Table 2.12: Animals

\subsubsection{Consumable supplies}

Consumable supplies were obtained from BD (Franklin Lakes, NJ, USA), Biozym (Hessisch Oldenburg, Germany), Eppendorf (Hamburg, Germany), Greiner Bio-One (Frickenhausen, Germany), Merck Millipore (Billerica, MA, USA), Sarstedt (Nümbrecht, Germany), Starlab (Hamburg, Germany) and Thermo Fisher Scientific, Nunc (Langenselbold, Germany).

\subsubsection{Equipment}

Standard laboratory equipment was used. Special instruments are listed below. They were run with the recommended software for each instrument.

\begin{tabular}{|l|l|}
\hline Instrument & Manufacturer \\
\hline \hline Applied Biosystems 7500 Real Time PCR System & Life Technologies, Darmstadt, Germany \\
\hline BioPhotometer & Eppendorf, Hamburg, Germany \\
\hline IX51, inverted microscope & Olympus, Hamburg, Germany \\
\hline Mikro-Dismembrator S & Sartorius, Göttingen, USA \\
\hline Mini-PROTEAN System, electrophoresis & BIO-RAD, München, Germany \\
\hline qTower, Real Time PCR System & Analytik Jena, Jena, Germany \\
\hline Semi-dry electroblotter & Peqlab, Erlangen, Germany \\
\hline Synergy HT, plate reader & BIO-TEK, Bad Friedrichshall, Germany \\
\hline Thermocycler T3000 & Biometra, Göttingen, Germany \\
\hline UV-Transilluminator & Biometra, Göttingen, Germany \\
\hline X-Ray processor, SRS-101A & Konica Minolta, Langenhagen, Germany \\
\hline
\end{tabular}

Table 2.13: Equipment 


\subsection{Methods}

\subsubsection{Cell biology}

Cell culture works were carried out under aseptic conditions in a laminar air flow work station.

\subsubsection{Cell cultivation}

Cells were grown in medium (see table 2.5) in culture flasks in a $5 \% \mathrm{CO}_{2}$ atmosphere saturated with $\mathrm{H}_{2} \mathrm{O}$ at $37^{\circ} \mathrm{C}$. Reaching a confluency up to $90 \%$, cells were passaged. Thus, medium was removed and cells were washed with PBS. Trypsin was added and cells were incubated at $37^{\circ} \mathrm{C}$ for detachment. Culture medium was added to inactivate trypsin. Cell suspension was centrifuged and resuspended in experimental medium for experiments or in cell culture medium in dilutions for further cultivation (see table 2.5).

\subsubsection{Freezing and thawing of cells}

After harvesting, cells were resuspended in freezing medium (see table 2.5) and aliquoted in cryo vials. Vials were put in a freezing device containing isopropyl alcohol for slow cooling and freezing at $-80^{\circ} \mathrm{C}$. Thereafter, vials were stored in liquid nitrogen.

Starting with a new stock of cells, a cryo vial was taken from liquid nitrogen. Cells were thawn by resuspension in warm culture medium (see table 2.5). Freezing medium was removed by centrifugation. Cells were resuspended in culture medium and transferred to culture flasks for cultivation.

\subsubsection{Determination of cell density}

Cell density was measured by a Neubauer counting chamber. $10 \mu$ of cell suspension was added and cells were counted. Cell number was given by the following equation:

$$
\frac{\text { number of cells }}{\text { number of squares }} \times 10^{4}=\frac{\text { cells }}{\mathrm{ml}}
$$

\subsubsection{Cocultivation}

For studying bone metastasis in vitro, cell culture models for bone-directed invasion and migration were used. 


\subsubsection{Invasion assay}

As in vitro model for bone directed invasion, breast cancer cells were cocultivated with osteoblastlike osteosarcoma cells without direct cell-cell contact in modified Boyden chambers (see figure 2.1). Matrigel ${ }^{T M}$ was used to represent the extracellular matrix [von Alten et al., 2006].

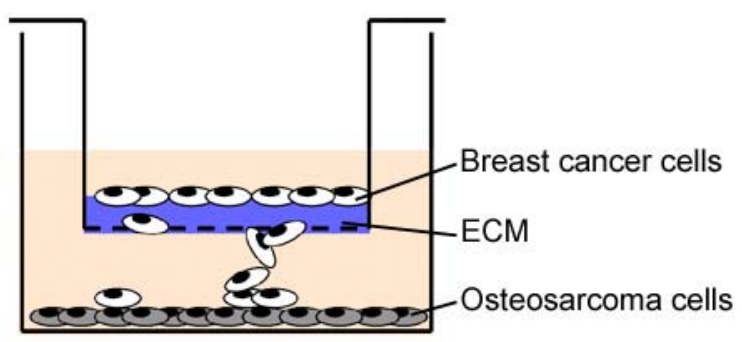

Figure 2.1: In vitro model for invasion studies under coculture conditions

Invasion was studied in a modified Boyden chamber assay. In the bottom well, osteosarcoma cells were grown. On top, breast cancer cells were seeded on an insert with a Matrigel ${ }^{T M}$ coated filter membrane. Both cell kinds were cultivated without direct cell-cell contact. An interaction was only possible by exchange of substrates through medium. ECM, extracellular matrix.

Cell culture inserts $(\varnothing 8 \mu \mathrm{m})$ were coated with Matrige ${ }^{T M}(1: 1$ solution in DMEM) on ice. For hardening, inserts were incubated at $37^{\circ} \mathrm{C}$ for $30 \mathrm{~min}$. Thereafter, breast cancer cell suspension was placed on top. Osteosarcoma cells were seeded separately in well plates. All of the cells were cultured in experimental medium (see table 2.5) at $37^{\circ} \mathrm{C}$ over night. The next day, inserts with breast cancer cells were set on top the osteosarcoma cells in well plates. Cells were cocultured up to $96 \mathrm{~h}$ at $37^{\circ} \mathrm{C}$.

Experiments were done in 6-well plates for generating RNA samples and in 12-well plates for invasion studies. Every experimental setting included monoculture controls, where the insert was put on top medium without osteosarcoma cells. For TGF $\beta_{1}$ studies, inserts were incubated with $2 \mu \mathrm{MTGF} \beta_{1}$. The enzyme was added at coculture begin. For KP-10 studies, inserts were incubated with different concentrations twice daily starting at coculture begin.

RNA samples were taken of the cells in the upper well. Therefore, Matrigel ${ }^{T M}$ together with the cells was collected and RNA was isolated (see chapter 2.2.3.1.1).

For invasion studies, inserts were washed with PBS and $\mathrm{H}_{2} \mathrm{O}$. Cells were fixed with $\mathrm{MeOH}$ and stained with haematoxylin. Matrigel ${ }^{T M}$ and cells on top the filter membrane were removed. Filters with the remaining cells at the bottom were cut of the inserts and placed on a microscope slide. Cells were counted. 


\subsubsection{Wound healing assay}

Migration was studied under coculture conditions. Therefore, petri dishes with a center well were used (see figure 2.2).

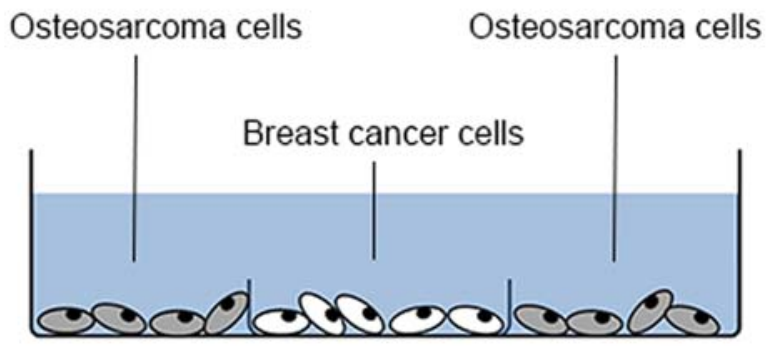

Figure 2.2: In vitro model for migration studies under coculture conditions

Migration was studied in a petri dish with a center well. In the outer circle, osteosarcoma cells were grown. In the middle, breast cancer cells were seeded. Both cell kinds were cultivated without direct cell-cell contact. An interaction was only possible by exchange of substrates through the medium.

Osteosarcoma cells were grown in the outer circle and breast cancer cells in the inner one. Cell suspension was filled in, so that it did not reached the inner wall. All of the cells were cultured in experimental medium (see table 2.5). Cells were incubated without contact at $37^{\circ} \mathrm{C}$ until the breast cancer cell layer was confluent. Then, a scratch was done by the bottom end of a pipette tip. Pictures of the scratch were taken directly after the scratch and $8 \mathrm{~h}, 24 \mathrm{~h}$ and $48 \mathrm{~h}$ later. The width of the scratch was measured. Monoculture controls were prepared comparably without osteosarcoma cells in the outer circle.

\subsubsection{Molecular biology}

\subsubsection{Isolation of ribonucleic acids (RNA)}

RNA was isolated by two different methods according to the requirements of further applications. A spin-column based method was used for isolation of mRNA for PCR analysis. The TriFast ${ }^{\text {TM }}$ reagent (PeqLab, Erlangen, Germany) was used for isolation of mRNA for gene array analysis.

\subsection{Spin-column method}

Total RNA was prepared by the RNeasy Mini Kit protocol according to the manufacturer's description (Qiagen, Hilden, Germany).

Cell culture samples and cell samples in Matrigel ${ }^{T M}$ were lysed and cleaned up in columns and RNA was purified by selective binding to silica membranes. After several washing steps, RNA was eluted from the membrane. 
The concentration of RNA in each sample was determined by photospectroscopy. Protein contamination were detected by the ratio of the optical densities of nucleic acids $\left(\mathrm{OD}_{260}\right)$ and of proteins $\left(\mathrm{OD}_{280}\right)$. Samples were further used, when the value was between 1.8 and 2.0.

RNA samples were stored at $-80^{\circ} \mathrm{C}$.

\subsection{TriFast $^{\mathrm{TM}}$ reagent}

For genearray analysis, isolation of RNA from invasion experiments was handled by a phenolchloroform based extraction [Chomczynski and Sacchi, 1987] with TriFast ${ }^{\text {TM }}$ reagent.

Cells below the filter membrane (see chapter 2.2.2.1) were washed with PBS after removal of medium and Matrige ${ }^{T M}$. Membranes were cut out of the cell culture inserts and put in TriFast ${ }^{\mathrm{TM}}$ reagent. After a few minutes, membranes were removed and the samples were directly delivered to the Transkriptomanalyselabor (TAL) in Göttingen for microarray analysis.

After return, RNA samples were stored at $-80^{\circ} \mathrm{C}$.

\subsubsection{Reverse transcription}

The RNA samples were treated with DNase I for digestion of DNA. For further analysis, RNA was reverse transcribed in complementary DNA ( $D$ DNA).

During synthesis of cDNA, a poly-T oligonucleotide primer hybridizes with a single strand RNA molecule. The enzyme reverse transcriptase synthesizes a complementary DNA strand depending on the RNA template [Verma et al., 1974].

DNA digestion was carried out by incubation of $1 \mu \mathrm{g}$ total RNA with $10 \mathrm{U}$ DNase I and $40 \mathrm{U}$ RNase inhibitor at $37^{\circ} \mathrm{C}$ for $30 \mathrm{~min}$. After $3 \mathrm{~min}$ at $70^{\circ} \mathrm{C}$, a reaction mixture was added to a final volume of $40 \mu$ containing $90 \mathrm{pmol}$ Primer $\mathrm{p}(\mathrm{dT}) 15,40 \mathrm{nmol}$ dNTPs and $400 \mathrm{U}$ Superscript ${ }^{\mathrm{TM}}$ ॥ reverse

transcriptase, 5x First-Strand buffer, $400 \mathrm{nmol}$ DTT. The preparation was heated up to $37^{\circ} \mathrm{C}$ for $60 \mathrm{~min}$ for elongation and transcription followed by $95^{\circ} \mathrm{C}$ for $5 \mathrm{~min}$ for enzyme inactivation. Negative controls were carried along with the samples.

cDNA samples was stored at $-20^{\circ} \mathrm{C}$.

\subsubsection{Polymerase chain reaction (PCR)}

PCR is a method for amplifying a certain gene product [Mullis et al., 1986]. It is organized in three steps: denaturation, annealing and elongation. During denaturation, double stranded DNA is separated in single strands by high temperature. Lower temperatures allows sequencespecific oligonucleotide primers to anneal to the certain gene product (template). Elongation of 
the primer-DNA structure is carried out by a polymerase by heating and a double strand DNA is generated. One PCR cycle is run through and the next begins again with denaturation. According to this, the amount of PCR product doubles with each cycle.

cDNA templates were amplified in a $15 \mu$ l reaction volume containing $0.3 \mathrm{U}$ KAPA2GTM Fast $(2 \mathrm{x}$

ReadyMix with Dye) and $0.5 \mu \mathrm{M}$ of the appropriate primers in a thermal cycler (T3000, Biometra). The initial denaturation step was carried out for $3 \mathrm{~min}$ at $95^{\circ} \mathrm{C}$. The cycles were performed at $95{ }^{\circ} \mathrm{C}$ for $15 \mathrm{~s}, 15 \mathrm{~s}$ at the primer-specific temperature and $5 \mathrm{~s}$ at $72{ }^{\circ} \mathrm{C}$. The number of cycles was adjusted to the amount of PCR product. End of the program was performed for 2 min at $72{ }^{\circ} \mathrm{C}$. No template controls were carried along with the samples.

For semi quantitative analysis, each sample was analyzed for the amount of L7. L7 was used as ribosomal housekeeping gene for standardization.

\subsubsection{Agarose gel electrophoresis}

For separation of DNA fragments, agarose gel electrophoresis was used.

DNA molecules are sorted by molecule based size and charge. By applying an electric field, molecules move through the gel in a proper buffer system. Smaller ones move faster than larger DNA fragments. The separated bands can be visualized by ethidium bromide staining an UV radiation, which intercalates into double stranded DNA.

The density of the agarose gel was adapted to the expected product length and varied between $1.5 \%$ and $2 \%\left(\mathrm{w} / \mathrm{v}\right.$ ) in $\frac{1}{2} \times$ TBS buffer (see table 2.4 ). Gels were casted $30 \mathrm{~min}$ for use. A proper DNA ladder was applied to the gels for length determination. Electrophoresis was performed in $\frac{1}{2} \times$ TBS buffer at $80-90 \mathrm{~V}$ for 30-75 min. PCR products were visualized by ethidium bromide staining and UV photometric detection. Bands were analyzed using the Biometra BioDoc Analyze System (Biometra, Göttingen, Germany).

\subsubsection{Microarray analysis}

Microarray analysis was performed by the Transkriptomanalyselabor (TAL) in Göttingen. Therefore, RNA samples were prepared (see chapter 2.2.3.1.2). Analysis were done by Affymetrix GeneChip ${ }^{\circledR}$ and raw data were prepared by $\mathrm{TAL}$ (see http://www.microarrays.med.uni-goettingen.de/; last page view on March 2013).

\subsubsection{Isolation of deoxyribonucleic acid (DNA)}

DNA was isolated by the NucleoSpin ${ }^{\circledR}$ Tissue Kit protocol according to the manufacturer's description (Macherey-Nagel, Dueren, Germany). 
For preparation of DNA from mouse tissue, bone samples were homogenized by a dismembrator. Lung and liver were cut by scalpel. Blood and cell samples were used directly. RNase was added. Lysates from bone, lung and liver were separated by NucleoSpin ${ }^{\circledR}$ Filters (Macherey-Nagel, Düren, Germany).

The concentration of DNA in each sample was measured by photospectroscopy and contamination were detected by the ratio of the optical densities of nucleic acids $\left(\mathrm{OD}_{260}\right)$ and of proteins $\left(\mathrm{OD}_{280}\right)$. A value between 1.8 and 2.0 was optimal. Because of a limited sample yield, all samples were used for further analysis. The results were interpreted based on the quality of the DNA samples.

DNA samples were stored at $-80^{\circ} \mathrm{C}$.

\subsubsection{Quantitative real-time PCR}

Quantitative real-time PCR is a method for amplifying DNA molecules according to the principles of a standard PCR (see chapter 2.2.3.3) and simultaneous quantification of the product. Compared to the standard PCR, where the product is detected in the end, real-time PCR enables detection as the reaction progresses. The accumulation of DNA product is measured by the increase of fluorescent dyes for every cycle. The dyes can be divided in groups with different detection methods. The intercalating dyes are non-specific fluorescent dyes, which intercalate with double stranded DNA [Higuchi et al., 1993]. The other group is characterized by sequence-specific probes that are labeled. Three methods are used. The TaqMan method uses a sequence-specific probe. The probe is labeled with a reporter dye and a quencher, which permits detection until the target is elongated. During elongation the reporter dye and the quencher are cut of and fluorescence can be detected [Livak et al., 1995]. Molecular beacon probes are characterized by complementary 5'- and 3'-ends, which form hairpin structures and offers low distances between reporter and quencher. A fluorescent signal is only detectable during hybridization of the molecular beacon with the target DNA [Tyagi and Kramer, 1996]. The method hybridization probes is based on two oligonucleotides, one having the acceptor at its 3'-end, the other having the donor at its 5'-end. Both oligonucleotides bind the same DNA strand with a maximum distance of five nucleotides. During hybridization, the fluorescent signal of the donor is detectable.

\subsection{SYBR green method}

For validation of the results of the micro array, the RNA samples were analyzed by real-time PCR and DNA products were quantified. Reverse transcription and real-time PCR were performed in one step.

$10 \mathrm{ng}$ RNA was added to $10 \mu \mathrm{l}$ 2x SensiFAST SYBR No-ROX One-Step Mix, $0.2 \mu$ reverse transcriptase, $0.4 \mu \mathrm{l}$ RNase inhibitor, $10 \mu \mathrm{M}$ sense and antisense primer respectively, in a final volume 
of $20 \mu \mathrm{l}$. The real-time PCR program was the following: $10 \mathrm{~min}$ at $45^{\circ} \mathrm{C}$ (reverse transcription), $2 \mathrm{~min}$ at $95^{\circ} \mathrm{C}$ (polymerase activation), followed by 40 cycles of $5 \mathrm{~s}$ at $95^{\circ} \mathrm{C}$ (denaturation) and $20 \mathrm{~s}$ at $59^{\circ} \mathrm{C}$ (annealing and elongation). The qTower (AnalytikJena) was used. One real-time PCR run was done in three replicates for each sample on 96-well plates. No template controls were performed for every primer.

Because of the limited RNA samples, no DNA digestion was performed. To avoid replication of DNA next to RNA, only primers with exon overlapping sequences were used.

The data were analyzed by qPCRsoft. Threshold and baseline were set automatically for each gene. Data were standardized to the expression of YWHAZ and normalized to the sample without treatment using the $\mathrm{ddCt}$ method. YWHAZ belongs to the 14-3-3 family and mediates signal transduction by binding to phosphoserine-containing proteins. It was used as housekeeping gene according to studies in literature showing stable expression in MCF-7 cells [Curtis et al., 2010, Chua et al., 2011, Ferreira and Cronjé, 2012].

\subsection{TaqMan method}

Samples of the in vivo studies were analyzed by real-time PCR using the TaqMan assay. The amount of human DNA in murine tissue was quantified by a duplex real-time PCR.

Target sequence was the ALU element, which is specific for primate genomes [Batzer and Deininger, 2002]. The ALU probe was labeled with VIC (Yakima Yellow) and quenched by a non-fluorescent dye, Black Hole Quencher 1 [Munoz et al., 2005]. As endogenous reference the 18S rRNA sequence was used for both, human and murine DNA. The $18 \mathrm{~S}$ rRNA probe was flagged with FAM (6-carboxyfluorescein) and TAMRA (carboxytetramethylrhodamine) as quencher.

Amplifications were carried out in a $25 \mu$ l reaction mixture containing $12.5 \mu \mathrm{l} 2 \mathrm{x}$ TaqMan ${ }^{\circledR}$ Universal PCR Master Mix, $22.5 \mathrm{pmol}$ of ALU sense and antisense primer respectively, $6.25 \mathrm{pmol}$ ALU probe, 15 pmol 18S rRNA primer pair, 3.125 pmol 18S rRNA probe and 50 ng DNA sample. Reactions were run on a thermal cycler (ABI 7500, Life Technologies) and conditions were set as $50^{\circ} \mathrm{C}$ for $2 \mathrm{~min}$ and $95^{\circ} \mathrm{C}$ for $10 \mathrm{~min}$ followed by 40 cycles of $95^{\circ} \mathrm{C}$ for $15 \mathrm{~s}$ and $60^{\circ} \mathrm{C}$ for $1 \mathrm{~min}$. One real-time PCR run was done in three replicates for each sample on 96-well plates and standard solutions for ALU and 18s rRNA were carried along. No template controls were performed.

The fluorescence intensity for VIC and FAM was aligned with ROX (6-carboxy-X-rhodamine) as passive reference. Data was analyzed by SDS software. Baseline and threshold were verified manually. The baseline was set a few cycles (2-3) before the cycle number where increasing fluorescence was detected. The threshold was placed in the exponential phase according to the fluorescent noise. Standard curves for ALU and 18s rRNA were calculated and the data were standardized. 


\subsubsection{Protein biochemistry}

\subsubsection{Immune cytochemical staining}

Cells were grown up to $70 \%$ confluency on two-well chamber slides in culture medium (see table 2.5). Before each treatment, cells were rinsed with PBS. Cells were fixed in $4 \%$ paraformaldehyde, treated with $3 \% \mathrm{H}_{2} \mathrm{O}_{2}$ and incubated with blocking solution (Histostain ${ }^{\circledR}$ Bulk Kit). Antibody dilution was added and cells were incubated at $4{ }^{\circ} \mathrm{C}$ overnight before they were again treated with Histostain ${ }^{\circledR}$ Bulk Kit according to the manufacturer's description. As detection reagent AEC substrate chromogen (Dako) was used. Controls were performed by omission of the primary antibody.

\subsubsection{Western blot analysis}

\subsection{Preparation of cell lysates}

Cell samples were collected from cultivated cells. Cell pellets were washed with PBS and resuspended in CelLytic ${ }^{\mathrm{TM}}$ buffer containing protease inhibitor. Samples were stored at $-80^{\circ} \mathrm{C}$.

For further preparation, lysates were thawn. Thereafter, samples were centrifuged at $4{ }^{\circ} \mathrm{C}$ for $5 \mathrm{~min}$ and supernatants were collected. Protein concentration was determined (see chapter 2.2.4.2.2). Equal amounts of protein per sample were diluted with $4 \times$ LDS sample buffer supplemented with $10 x$ sample reducing agent. Samples were denaturated at $95^{\circ} \mathrm{C}$ for $10 \mathrm{~min}$. Protein samples were directly applied to gel electrophoresis (see chapter 2.2 .4 .2 .3 ) or stored at $-20^{\circ} \mathrm{C}$.

\subsection{Protein determination}

The concentration of solubilized protein was determined with the Bio-Rad Protein Assay based on the Bradford method [Bradford, 1976]. Adding a specific dye to a protein solution changes the color by binding to the protein. Color shift occurs in response to the concentration of protein.

Bio-Rad Protein Assay reagent was diluted in $\mathrm{H}_{2} \mathrm{O}(1: 5)$ and filled in 96-well plates. On top, samples and BSA standards were added. The plates were measured by a microplate reader at $590 \mathrm{~nm}$. BSA standard curve was calculated and protein concentrations of the samples were detected. Experiments were done in three replicates for each sample and standard.

\subsection{Polyacrylamide gel electrophoresis}

Proteins were separated by sodium dodecyl sulfate polyacrylamide gel electrophoresis (SDS-PAGE) [Laemmli, 1970]. Proteins bind to SDS and the resulting complexes are negatively charged in a 
ratio of charge to mass. The complexes are sorted by size in the gel by applying an electric field in a proper buffer system. Smaller ones move faster than larger protein complexes.

Gels were separated in two areas: on top a gel for protein collection with $5 \%$ density and underneath, a gel for separation, which density was adapted to the expected protein mass $(10 \%, 12 \%$ or $14 \%$; see table 2.14 ).

\begin{tabular}{|l|l|l|l|l|}
\hline & Collection (5\%) & $\begin{array}{l}\text { Separation } \\
(10 \%)\end{array}$ & $\begin{array}{l}\text { Separation } \\
(12 \%)\end{array}$ & $\begin{array}{l}\text { Separation } \\
(14 \%)\end{array}$ \\
\hline \hline $\begin{array}{l}\text { ProSieve }{ }^{\circledR} 50 \text { Gel solution } \\
\text { (acrylamide) }\end{array}$ & $500 \mu \mathrm{l}$ & $2.0 \mathrm{ml}$ & $2.4 \mathrm{ml}$ & $2.8 \mathrm{ml}$ \\
\hline Sol B & - & $2.5 \mathrm{ml}$ & $2.5 \mathrm{ml}$ & $2.5 \mathrm{ml}$ \\
\hline Sol C & $50 \mu \mathrm{l}$ & $100 \mu \mathrm{l}$ & $100 \mu \mathrm{l}$ & $100 \mu \mathrm{l}$ \\
\hline Sol D & $1.3 \mathrm{ml}$ & - & - & - \\
\hline $\mathrm{H}_{2} \mathrm{O}$ & $3.1 \mathrm{ml}$ & $5.3 \mathrm{ml}$ & $4.9 \mathrm{ml}$ & $4.5 \mathrm{ml}$ \\
\hline TEMED & $5 \mu \mathrm{l}$ & $5 \mu \mathrm{l}$ & $5 \mu \mathrm{l}$ & $5 \mu \mathrm{l}$ \\
\hline APS $(10 \%)$ & $50 \mu \mathrm{l}$ & $50 \mu \mathrm{l}$ & $50 \mu \mathrm{l}$ & $50 \mu \mathrm{l}$ \\
\hline
\end{tabular}

Table 2.14: Composition for two gels

First, gel for separation was prepared and polymerized for $45 \mathrm{~min}$. Gel for collection was casted on top and polymerized for $30 \mathrm{~min}$. Gels were directly used for electrophoresis or stored in a humid atmosphere at $4{ }^{\circ} \mathrm{C}$ up to three days.

Samples in LDS sample buffer and protein markers were loaded into the gel slots. Electrophoresis was performed in SDS electrophoresis buffer for $30 \mathrm{~min}$ at $80 \mathrm{~V}$ for collection, followed by $45 \mathrm{~min}$ $130 \mathrm{~min}$ at $150 \mathrm{~V}$ for separation. Gels were equilibrated in transfer buffer.

\subsection{Blotting}

Proteins were transferred to a membrane after electrophoresis. A semi-dry electroblotter was used. Therefore, four chromatography papers were bathed with concentrated anode buffer. On top, four chromatography papers were placed and bathed with anode buffer. Gels were applied. PVDF membranes were activated in $\mathrm{MeOH}$, washed in $\mathrm{H}_{2} \mathrm{O}$, equilibrated in transfer buffer and then, put on top of the gels. Again, four chromatography papers were bathed in cathode buffer and placed above. Gels were blotted for $90 \mathrm{~min}-105 \mathrm{~min}$ at $100 \mathrm{~V}-110 \mathrm{~V}$ and $4{ }^{\circ} \mathrm{C}$. After blotting, membranes were equilibrated in TBST.

\subsection{Immunostaining}

Proteins were detected by immunostaining. Therefore, membranes were blocked for 60 min with blocking solution for saturation at all places, where no protein has been attached. Then, membranes were rinsed with TBST and incubated in a solution of the primary antibody over night at 
$4{ }^{\circ} \mathrm{C}$. The next day, membranes were washed with TBST and incubated in a solution of the secondary antibody for $1 \mathrm{~h}$ at room temperature. The secondary antibody was coupled to horseradish peroxidase allowing chemiluminescent detection. After TBST washing steps, membranes were exposed to the Immobilion ${ }^{\mathrm{TM}}$ Western detection solution. Light emission of the membranes was detected by X-ray film in a X-ray processor at the site of specific antibody binding. Bands were analyzed using the Biometra BioDoc Analyze System (Biometra, Göttingen, Germany).

\subsubsection{Proliferation assay}

Cells were harvested and sowed in 96 well-plates in experimental medium (see table 2.5 ) at $37^{\circ} \mathrm{C}$ overnight. Cell density varied between $1^{*} 10^{4}$ and $2 * 10^{4}$ cells per well depending on the metabolism of each cell line. KP-10 solutions and vehicle (control) were added once daily. alamarBlue ${ }^{\circledR}$ solution was added after $72 \mathrm{~h}$ and cells were incubated for $4 \mathrm{~h}$ respectively $7 \mathrm{~h}$. Then, the plates were measured by a microplate reader.

alamarBlue ${ }^{\circledR}$ (AbD Serotec, Oxford, UK) is an redox indicator. It is reduced by cellular metabolism processes and offers the possibility of an indirect measurement of viable cells. The colorimetric change from blue to violet is measured by the optical density of the reduced dye at $570 \mathrm{~nm}$ vs. $630 \mathrm{~nm}$ [Voytik-Harbin et al., 1998].

Experiments were done in six replicates for each sample. Changes in viability were used as marker for proliferation compared to control samples.

For transfected cell experiments, transfection stability was controlled by RT-PCR samples of cells growing in experimental or transfection media.

\subsubsection{Animal experiments}

Animal studies were approved and carried out according to the mandatory ethical guidelines and the German laws for protection of animals.

\subsubsection{Keeping of animals}

Six till eight weeks old female CD1 nude mice were kept in groups of three to five in cages. They were maintained in a $12 \mathrm{~h}$ light/dark schedule and were fed and watered ad libitum.

\subsubsection{Anesthesia}

Mice were anesthetized before tumor cell injection in the breast or intracardially. A mixture of ketamine [100 mg/kg BW], xylazine [4 mg/kg BW] in sodium chloride [0.9\%] was applied 
intraperitoneally in a final volume of $200 \mu$ per mouse.

\subsubsection{Tumor cell injection}

Different xenograft models were used. Therefore, breast cancer cells were injected intracardially ( $10^{5}$ cells per mouse) into the left ventrical or orthotopically at the second pair of mammary glands into the fatty tissue $\left(10^{6}\right.$ cells per gland).

Cells were prepared in MEM without additives. Depending on the cell line, Matrige ${ }^{T M}$ was added (1:1) for optimal growth conditions in vivo.

\subsubsection{Study design}

Mice of every in vivo study were divided into three groups: the first group without tumor cell injection, the second with tumor cell injection but without treatment, and the third group with tumor cell injection and treatment with $\mathrm{KP}-10$ [50 $\mathrm{\mu g} /$ mouse] daily starting the day after injection. Study end was determined by tumor volume.

\subsubsection{Tumor volume}

Tumor growth was measured as tumor volume. Equation 2.1 describes the volume of a spheroid with length, as longest dimension, and width, as the distance perpendicular to and in the same plane as the length [Richtig et al., 2004].

$$
\frac{1}{6} \pi \times \text { width }{ }^{2} \times \text { length }=\text { Tumorvolume }
$$

Tumors were measured two times a week.

\subsubsection{Section}

At the end of the study, mice were euthanized with $\mathrm{CO}_{2}$ and blood was collected by cardiocentesis. Lung, femurs and liver were taken.

For studies with no cardiocentesis, mice were euthanized with $\mathrm{CO}_{2}$ and cervical dislocation was done.

\subsubsection{Sample preparation}

Blood samples were added by EDTA $[4 \mathrm{mM}]$ and stored at $-20^{\circ} \mathrm{C}$. 
Collected murine tissue was washed with PBS. Bones were relieved of tissue. Samples were frosted in liquid nitrogen and stored at $-80^{\circ} \mathrm{C}$.

DNA was isolated (see chapter 2.2.3.6) and metastasis was measured (see chapter 2.2.3.7.2).

\subsubsection{Statistical analysis}

The statistical analysis was carried out by the GraphPad Prism ${ }^{\circledR}$ software 5.0. The results were graphed by mean \pm standard error of the mean (SEM). Significant differences were indicated by $*: \mathrm{p}<0.05, * *: \mathrm{p}<0.01$ and $* * *: \mathrm{p}<0.001$. The number of samples was specified as $\mathrm{n}$.

Cell culture experiments were done three times and more in independent preparations. In most of the studies, two different conditions respectively treatments were compared and the paired t-test was used for statistical analysis. Experimental settings covering more than two conditions respectively treatments, data was analyzed by a 1way ANOVA together with a multiple comparison test. Tukey's was used for comparison of all pairs of columns and Dunnett's was used, if every data column should be compared only with the control. Images were graphed in figures being representative for at least three sample preparations.

Data of the in vivo experiments was differently analyzed according to the compared groups. The Mann Whitney test was used for matching the control group versus the tumor group. In the control group, it was expected that no hDNA could be detected. Thus, a Gaussian distribution could not be estimated and the nonparametric test was used. In contrast, hDNA was expected to be found in the tumor group and in the treatment group. Here, the data was analyzed by the paired t-test. 


\section{Results}

\subsection{Morphology and motility marker expression of breast cancer cells}

In the following chapter, breast cancer cell lines (see table 2.9) were characterized by their morphology and their intracellular configuration of markers important for EMT.

\subsubsection{Morphological characteristics of breast cancer cells}

Breast cancer cell lines were studied for their morphology. Therefore, images of living cells were taken. The pictures in figure 3.1 are representative for each cell line. T47D, ZR75-1, MDA-MB-453, HCC 70, HCC 1806, HCC 1937 and MCF-7 showed growth in clusters with intercell connections like epithelial cells. MDA-MB-231 and MDA-MB-435s had a more spindle shaped appearance like mesenchymal cells. 
T47D

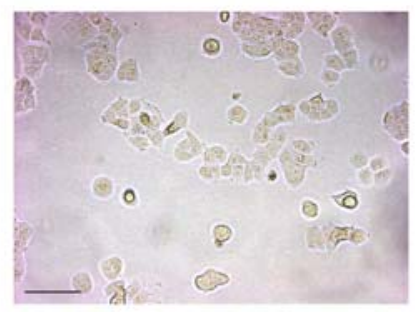

MDA-MB-231

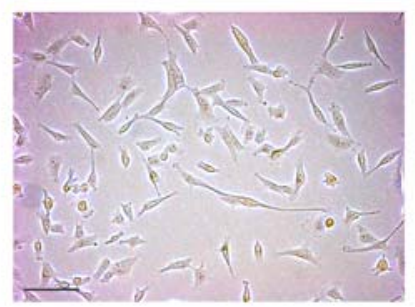

HCC 70

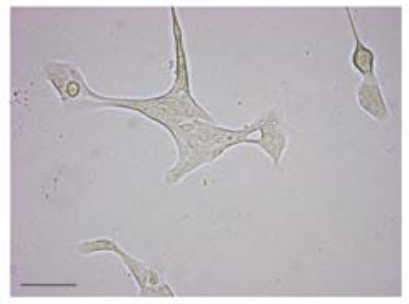

ZR75-1

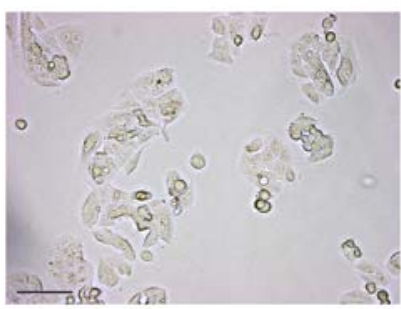

MDA-MB-435s

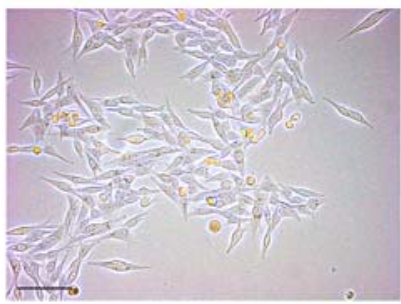

HCC 1806

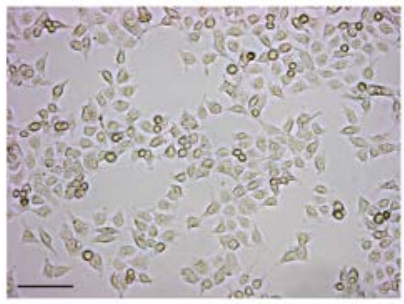

MCF-7

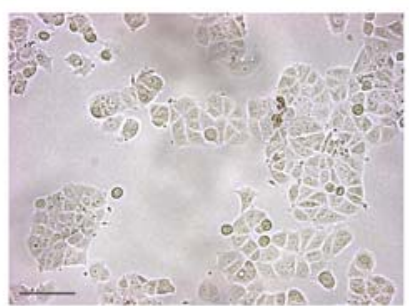

MDA-MB-453

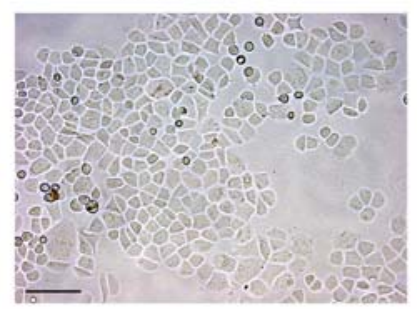

HCC 1937

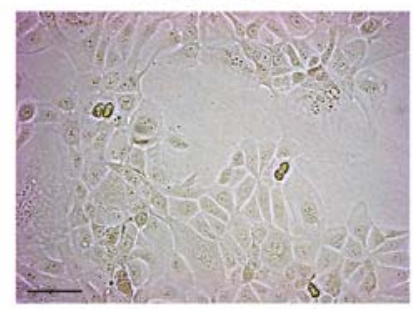

Figure 3.1: Morphology of breast cancer cells

Breast cancer cell lines T47D, ZR75-1, MCF-7, MDA-MB-231, MDA-MB-435s, MDA-MB-453, HCC 70, HCC 1806 and HCC 1937 were grown in culture flasks. Bright field images of living cells were taken (scale bar $=100 \mu \mathrm{m}$ ). Images represent the findings in at least three different passages of each cell line.

\subsubsection{EMT marker expression}

Breast cancer cell lines were further characterized for expression of genes involved in EMT by mRNA analysis (see chapters 2.2.3.1-2.2.3.4). As marker for the epithelial phenotype CDH1 and TJP1 were chosen. S100A4 and VIM were investigated as marker for the mesenchymal phenotype.

Figure 3.2 represents the expression levels of $\mathrm{CDH} 1$ and TJP1 (figure $3.2 \mathrm{a}$ ) and the expression levels of S100A4 and VIM (figure 3.2b). Epithelial marker TJP1 could be detected in all of the studied cell lines. CDH1 was expressed in almost all of the breast cancer cells. In MDA-MB-231, $\mathrm{CDH} 1$ was detected in a small amount. In MDA-MB-435s, no CDH1 was found. Mesenchymal markers were expressed differently in the studied cell lines. S100A4 was clearly identified in T47D, ZR75-1, MDA-MB-231, HCC 70, HCC 1806, HCC 1937 and MCF-7. MDA-MB-435s cells expressed only a small amount of S100A4. In MDA-MB-453, no S100A4 could be detected. VIM 
was found in MDA-MB-231, MDA-MB-435s, HCC 70, HCC 1937 and MCF-7, but not in T47D and ZR75-1. In HCC 1806 and MDA-MB-453 cells, VIM was expressed in a small amount.

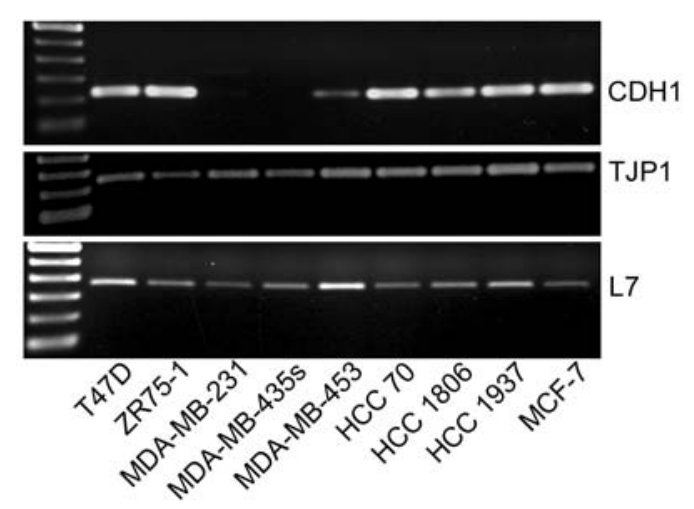

(a) Epithelial marker $\mathrm{CDH} 1$ and TJP1

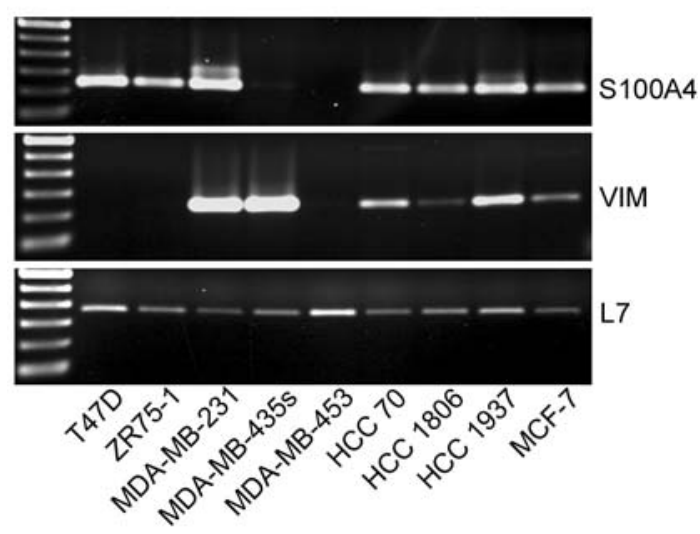

(b) Mesenchymal marker S100A4 and VIM

Figure 3.2: Expression of EMT marker in breast cancer cell lines

EMT marker distribution was investigated by gene expression analysis via RT-PCR. For reasons of comparison, mRNA controls were performed by proof of the housekeeping gene L7. (a) Levels of CDH1 and TJP1 were detected as marker for the epithelial phenotype. (b) S100A4 and VIM were picked as marker for the mesenchymal phenotype. Images represent the findings in at least three different passages of each cell line.

In summary, expression of epithelial marker CDH1 and TJP1 showed a different pattern within the cells lines. No differences were observed in TJP1 gene expression between the cell lines. All of the breast cancer cell lines showed a clear CDH1 expression except MDA-MB-231 and MDA-MB-435s cells. Mesenchymal marker S100A4 and VIM were expressed in HCC 70, HCC 1806, HCC 1937 and MCF-7 in detectable quantities. In T47D and ZR75-1, only S100A4 was verified. MDA-MB-231 cells had large amounts of S100A4 and VIM. MDA-MB-435s cells expressed clear amounts of VIM, but they had very low S100A4 expression. MDA-MB-453 cells showed very small quantities of VIM and no S100A4 expression.

\subsection{Invasion of breast cancer cells}

In this chapter, the breast cancer cell lines were analyzed for their invasion properties. Cell lines showing great differences in their invasive behavior were further analyzed.

\subsubsection{Invasive properties of breast cancer cells}

Invasive behavior of breast cancer cells was studied. Therefore, a modified Boyden chamber assay was chosen as model system (see chapter 2.2.2.1). Cells moved through a Matrigel ${ }^{T M}$ coated 
filter membrane towards medium in monoculture or towards osteosarcoma cells in coculture up to $96 \mathrm{~h}$.

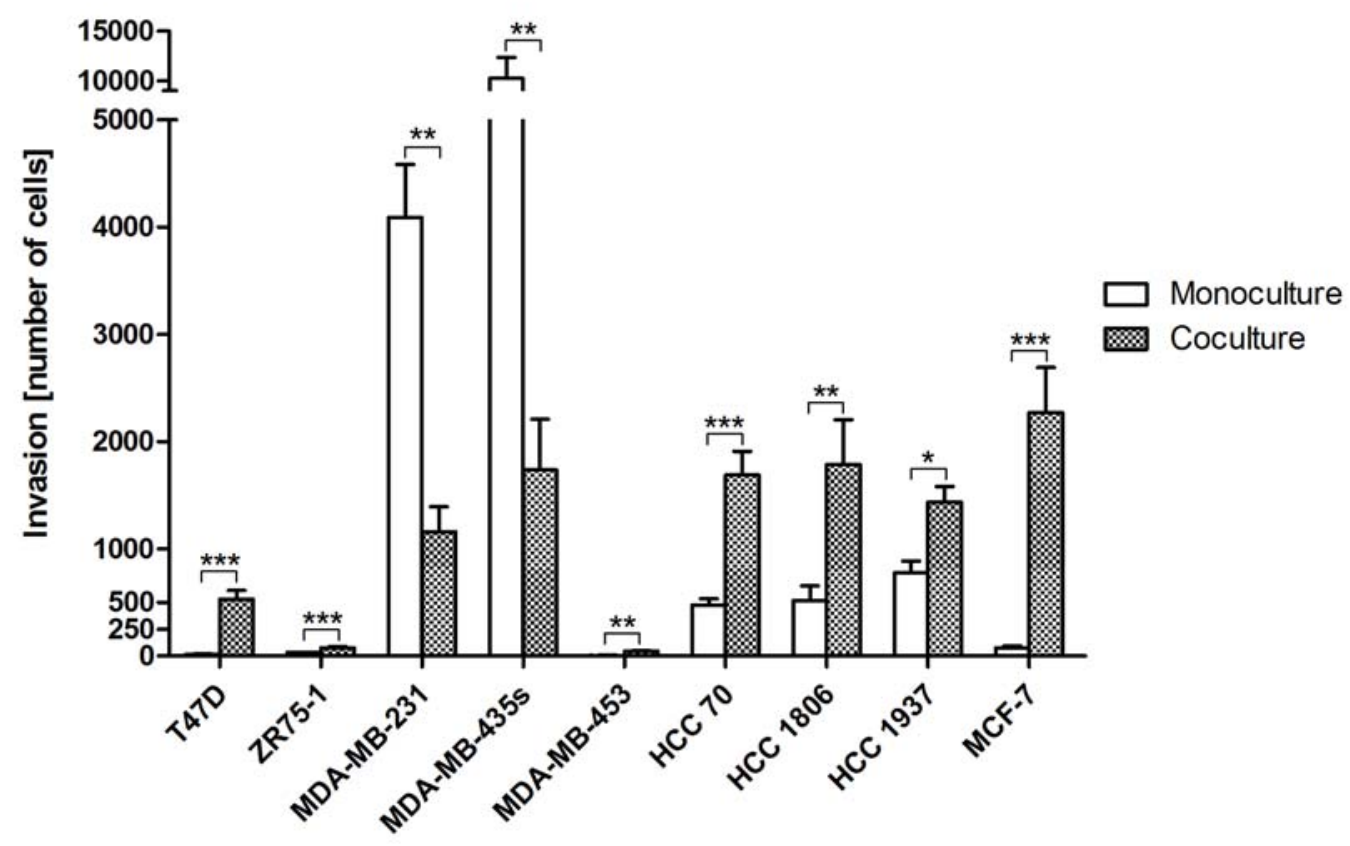

Figure 3.3: Invasive behavior of breast cancer cells

The breast cancer cell lines T47D, ZR75-1, MDA-MB-231, MDA-MB-435s, MDA-MB-453, HCC 70, HCC 1806, HCC 1937 and MCF-7 were studied on their invasive properties by a modified Boyden chamber assay. Numbers of invaded cells were collected in monoculture (white columns) and cocultivated with osteosarcoma cells (dotted columns) after $96 \mathrm{~h}$. Results were analyzed by paired t-test comparing data of monoculture and coculture conditions within one cell line (mean \pm SEM; *: $p<0.05 ; * *: p<0.01 ; * * *: p<0.001 ; n=6$ for MDA-MB-435s, HCC 1937; $\mathrm{n}=8$ for T47D, ZR75-1, MDA-MB-231, MDA-MB-453, HCC 70, HCC 1806; $\mathrm{n}=17$ for MCF-7).

In figure 3.3, the numbers of invaded cells are represented for all breast cancer cell lines under monoculture and coculture conditions.

In monoculture, MDA-MB-435s (10236 cells \pm 2125 cells SEM) and MDA-MB-231 (4093 cells \pm 493 cells SEM) showed strong invasion. T47D (16 cells \pm 4 cells SEM), ZR75-1 (30 cells \pm 5 cells SEM), MDA-MB-453 (5 cells \pm 2 cells SEM) and MCF-7 (73 cells \pm 21 cells SEM) rarely invaded. HCC 70 (473 cells \pm 58 cells SEM), HCC 1806 (518 cells \pm 136 cells SEM) and HCC 1937 (774 cells \pm 110 cells SEM) had intermediate invasive properties.

Under coculture conditions, a significant increase of invasion was observed for T47D (528 cells \pm 84 cells SEM), ZR75-1 (77 cells \pm 10 cells SEM), MDA-MB-453 (46 cells \pm 8 cells SEM), HCC 70 (1686 cells \pm 225 cells SEM), HCC 1806 (1784 cells \pm 420 cells SEM), HCC 1937 (1437 cells \pm 144 cells SEM) and MCF-7 (2268 cells \pm 421 cells SEM). MDA-MB-231 (1160 cells \pm 232 cells SEM) and MDA-MB-435s (1736 cells \pm 473 cells SEM) showed significantly reduced invasion in contrast to monoculture. 


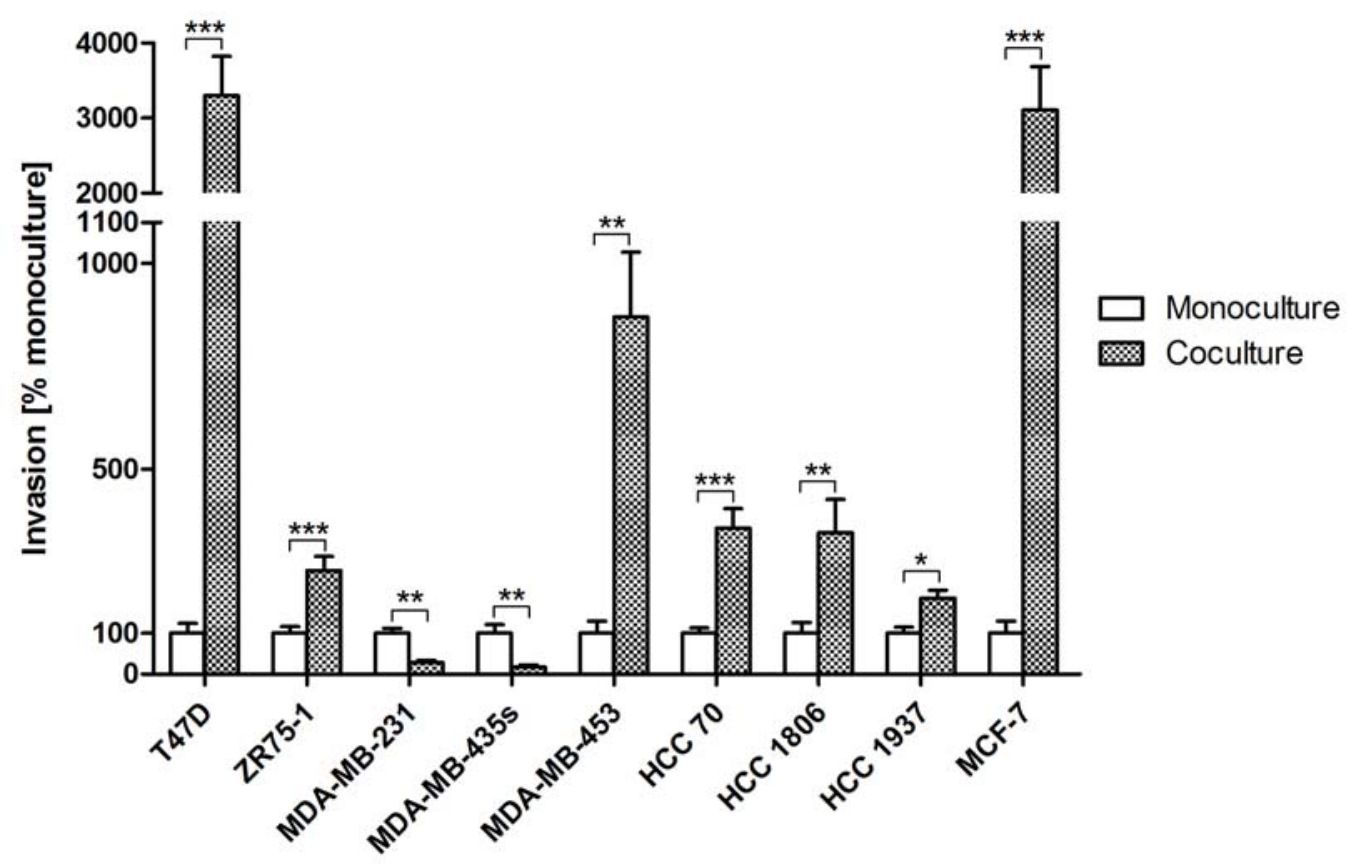

Figure 3.4: Invasion of breast cancer cells affected by cocultivation with osteosarcoma cells

The breast cancer cell lines T47D, ZR75-1, MDA-MB-231, MDA-MB-435s, MDA-MB-453, HCC 70, HCC 1806, HCC 1937 and MCF-7 were studied on their invasive properties by a modified Boyden chamber assay. Invasion of cells cocultivated with osteosarcoma cells (dotted columns) is graphed in relation to the invaded cells in monoculture (white columns) for each cell line after $96 \mathrm{~h}$. Results were analyzed by paired t-test comparing data of monoculture and coculture conditions within one cell line (mean $\pm \mathrm{SEM}$; $* \mathrm{p}<0.05 ; * *$ : $\mathrm{p}<0.01$; $* * *$ : $\mathrm{p}<0.001 ; \mathrm{n}=6$ for MDA-MB-435s, HCC 1937; $\mathrm{n}=8$ for T47D, ZR75-1, MDA-MB-231, MDA-MB-453, HCC 70, HCC 1806; $n=17$ for MCF-7).

For comparison of invasion within one cell line, figure 3.4 shows the extent of invasion in coculture related to the invasion in monoculture. MDA-MB-231 (28.3\% $\pm 5.7 \%$ SEM) and MDA-MB-435s $(17.0 \% \pm 4.6 \%$ SEM) were significantly inhibited in their invasion properties by cocultivation with osteosarcoma cells. T47D $(3297.7 \% \pm 523.8 \%$ SEM) and MCF-7 $(3108.8 \% \pm 577.0 \%$ SEM) showed the largest increase in invasion. ZR75-1 (252.3\% $\pm 34.5 \%$ SEM), MDA-MB-453 $(869.0 \% \pm 158.0 \%$ SEM), HCC $70(356.3 \% \pm 47.5 \%$ SEM), HCC $1806(344.5 \% \pm 81.1 \%$ SEM) and HCC $1937(185.8 \% \pm 18.6 \%$ SEM) also had a significant increase in invasion.

Taken together, the breast cancer cell lines showed different invasive behavior from hardly any invasion to a strong cell movement. Coculture conditions led to increased cell invasion especially in cell lines showing almost no invasion in monoculture as T47D and MCF-7 cells. The cell lines with the strongest invasion properties in monoculture, MDA-MB-231 and MDA-MB-435s, showed decreased cell movement in coculture. 


\subsubsection{Progression of bone-directed invasion}

Four different breast cancer cell lines were chosen for a detailed study of their invasion behavior. MDA-MD-231 and MDA-MB-435s cells were investigated as cell lines showing reduced invasion in coculture (see chapter 3.2.1). MCF-7 cells showed a strong increase in invasion by cocultivation with osteosarcoma cells. HCC 1806 cells had an intermediate increase in invasion. Invasion was studied for $24 \mathrm{~h}, 48 \mathrm{~h}, 72 \mathrm{~h}$ and $96 \mathrm{~h}$.

\subsubsection{Invasion of MDA-MD-231 and MDA-MB-435s}

Invasion of MDA-MB-231 cells is represented as number of invaded cells in figure 3.5. For up to $48 \mathrm{~h}$, cells in coculture showed significantly increased invasion compared to monocultured cells ( $24 \mathrm{~h}: 35$ cells \pm 11 cells SEM vs. 124 cells \pm 38 cells SEM; $48 \mathrm{~h}: 117$ cells \pm 26 cells SEM vs. 296 cells \pm 60 cells SEM). At $72 \mathrm{~h}$, no significant difference was observable between both conditions ( 620 cells \pm 155 cells SEM vs. 808 cells \pm 244 cells SEM). At $96 \mathrm{~h}$, cells in monoculture invaded stronger than cells in coculture (see chapter 3.2.1).

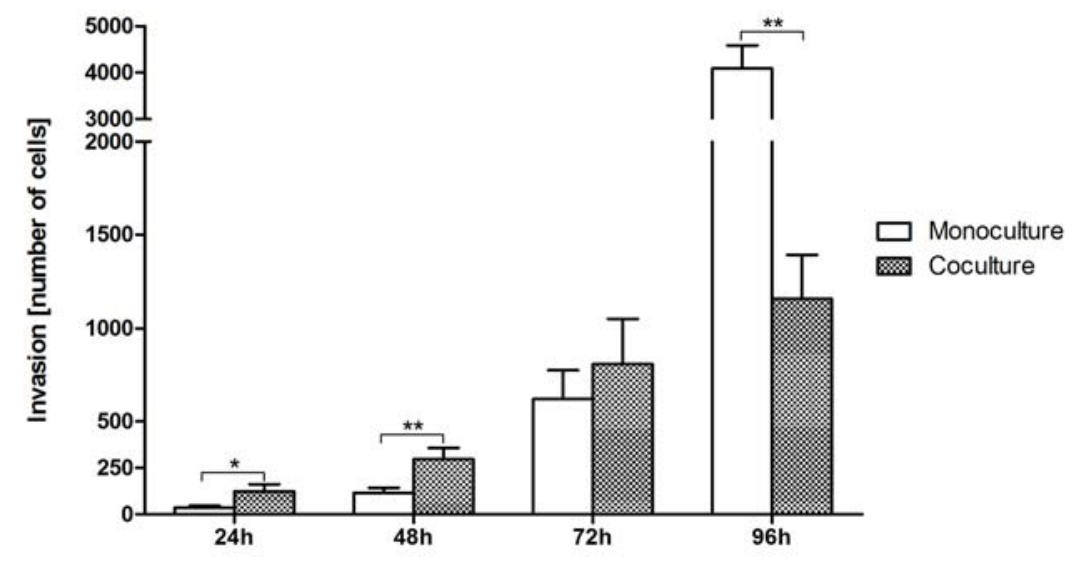

Figure 3.5: Invasive characteristics of MDA-MB-231 cells in coculture

Invasion of the breast cancer cell line MDA-MB-231 was studied by a modified Boyden chamber assay for cells in monoculture (white columns) and cells cocultivated with osteosarcoma cells (dotted columns). Numbers of invaded cells were counted after $24 \mathrm{~h}, 48 \mathrm{~h}, 72 \mathrm{~h}$ and $96 \mathrm{~h}$. Results were analyzed by paired t-test comparing data of monoculture and coculture conditions within one point in time (mean \pm SEM; *: $p<0.05 ; * *: p<0.01 ; n=8$ for $24 h, 48 h, 96 h ; n=10$ for $72 h$ ).

Invasion of MDA-MB-435s cells is graphed in figure 3.6. A significantly increased invasion was observed for up to $48 \mathrm{~h}$ ( $24 \mathrm{~h}: 6$ cells \pm 1 cells SEM vs. 27 cells \pm 4 cells SEM; $48 \mathrm{~h}$ : 27 cells \pm 7 cells SEM vs. 96 cells \pm 20 cells SEM). The later measurements showed less invasion in coculture than in monoculture ( $72 \mathrm{~h}: 725$ cells \pm 228 cells SEM vs. 501 cells \pm 172 cells SEM; $96 \mathrm{~h}: 10236$ cells \pm 2125 cells SEM vs. 1736 cells \pm 473 cells SEM). 


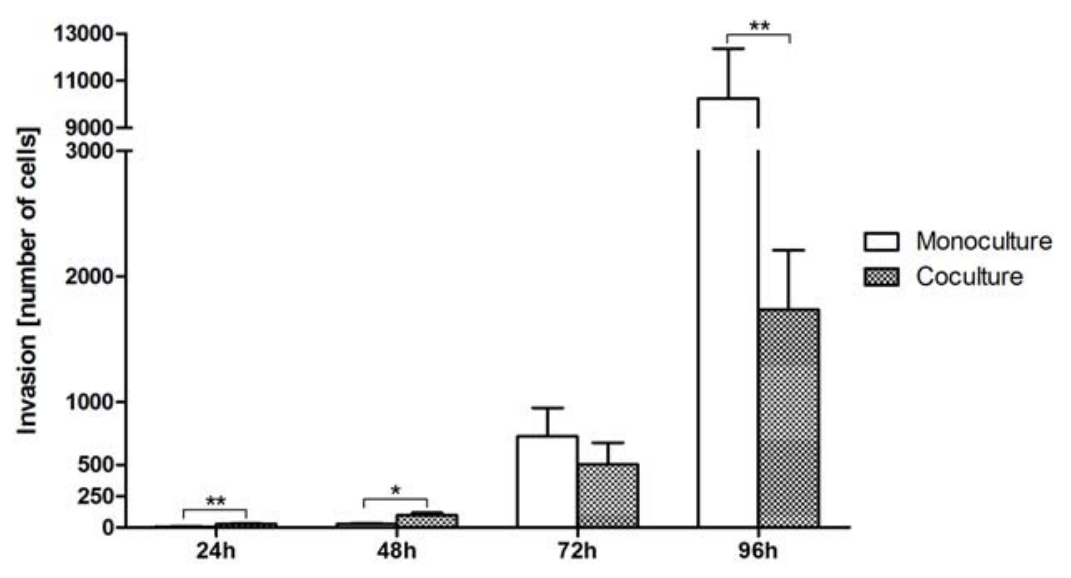

Figure 3.6: Invasive characteristics of MDA-MB-435s cells in coculture

Invasion of the breast cancer cell line MDA-MB-435s was studied by a modified Boyden chamber assay for cells in monoculture (white columns) and cells cocultivated with osteosarcoma cells (dotted columns). Numbers of invaded cells were counted after $24 \mathrm{~h}, 48 \mathrm{~h}, 72 \mathrm{~h}$ and $96 \mathrm{~h}$. Results were analyzed by paired t-test comparing data of monoculture and coculture conditions within one point in time (mean \pm SEM; $*: p<0.05 ; * *: p<0.01 ; n=8$ for $24 h ; n=7$ for $48 h ; n=11$ for $72 h ; n=6$ for $96 h$ ).

\subsubsection{Invasion of HCC 1806}

The cell line HCC 1806 showed a significantly increased invasion in coculture after $48 \mathrm{~h}, 72 \mathrm{~h}$ and $96 \mathrm{~h}$ graphed in figure 3.7 ( $24 \mathrm{~h}: 7$ cells \pm 1 cells SEM vs. 25 cells \pm 8 cells SEM; $48 \mathrm{~h}$ : 40 cells \pm 7 cells SEM vs. 265 cells \pm 50 cells SEM; $72 \mathrm{~h}$ : 429 cells \pm 89 cells SEM vs. 1413 cells \pm 323 cells SEM; $96 \mathrm{~h}$ : 518 cells \pm 136 cells SEM vs. 1784 cells \pm 420 cells SEM).

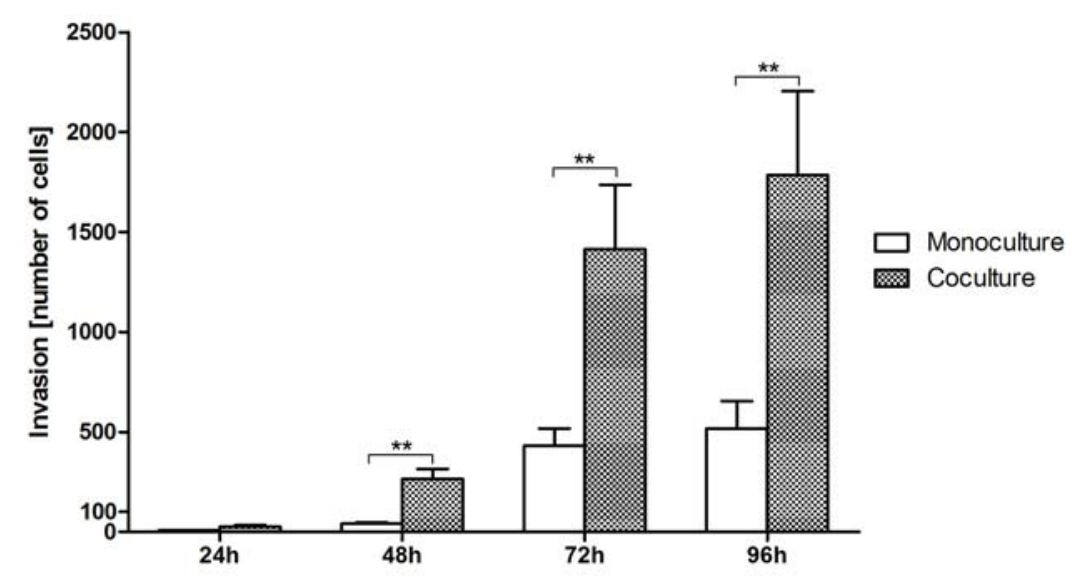

Figure 3.7: Invasive characteristics of HCC 1806 cells in coculture

Invasion of the breast cancer cell line HCC 1806 was studied by a modified Boyden chamber assay for cells in monoculture (white columns) and cells cocultivated with osteosarcoma cells (dotted columns). Numbers of invaded cells were counted after $24 \mathrm{~h}, 48 \mathrm{~h}, 72 \mathrm{~h}$ and $96 \mathrm{~h}$. Results were analyzed by paired t-test comparing data of monoculture and coculture conditions within one point in time (mean \pm SEM; **: $p<0.01 ; n=7$ for $24 \mathrm{~h}$; $\mathrm{n}=8$ for $48 \mathrm{~h}, 72 \mathrm{~h}, 96 \mathrm{~h}$ ). 


\subsubsection{Invasion and migration of MCF-7}

Invasion of MCF-7 is represented in figure 3.8. The cocultured cells invaded significantly stronger after $48 \mathrm{~h}, 72 \mathrm{~h}$ and $96 \mathrm{~h}(24 \mathrm{~h}: 15$ cells \pm 4 cells SEM vs. 28 cells \pm 10 cells SEM; $48 \mathrm{~h}: 21$ cells \pm 3 cells SEM vs. 74 cells \pm 6 cells SEM; $72 \mathrm{~h}$ : 69 cells \pm 35 cells SEM vs. 489 cells \pm 103 cells SEM; 96 h: 73 cells \pm 21 cells SEM vs. 2268 cells \pm 421 cells SEM).

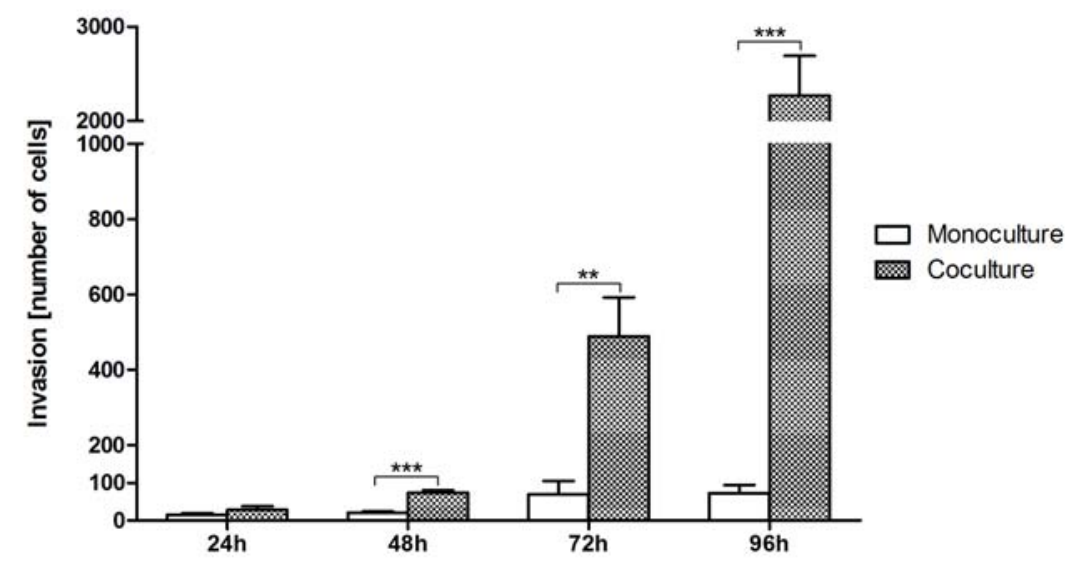

Figure 3.8: Invasive characteristics of MCF-7 cells in coculture

Invasion of the breast cancer cell line MCF-7 was studied by a modified Boyden chamber assay for cells in monoculture (white columns) and cells cocultivated with osteosarcoma cells (dotted columns). Numbers of invaded cells were counted after $24 \mathrm{~h}, 48 \mathrm{~h}, 72 \mathrm{~h}$ and $96 \mathrm{~h}$. Results were analyzed by paired t-test comparing data of monoculture and coculture conditions within one point in time (mean $\pm \mathrm{SEM} ; * * \mathrm{p}<0.01 ; * * * \mathrm{p}<0.001 ; \mathrm{n}=8$ for $24 h, 48 h, 72 h ; n=17$ for $96 h$ ).

MCF-7 cells were studied for their migration behavior in monoculture and coculture. Therefore, a wound healing assay was used (see chapter 2.2.2.2). The scratch was observed for up to $48 \mathrm{~h}$. In figure 3.9a pictures of the scratches in control and coculture samples are shown. Time point $0 \mathrm{~h}$ shows the scratches directly after the cell layer was wounded. The widths between the cell layers was measured after $8 \mathrm{~h}, 24 \mathrm{~h}$ and $48 \mathrm{~h}$ and the results are graphed in figure $3.9 \mathrm{~b}$. Cell migration is represented as decreasing width of the scratches. No difference in cell migration was seen in monocultured and cocultured cell layers. 


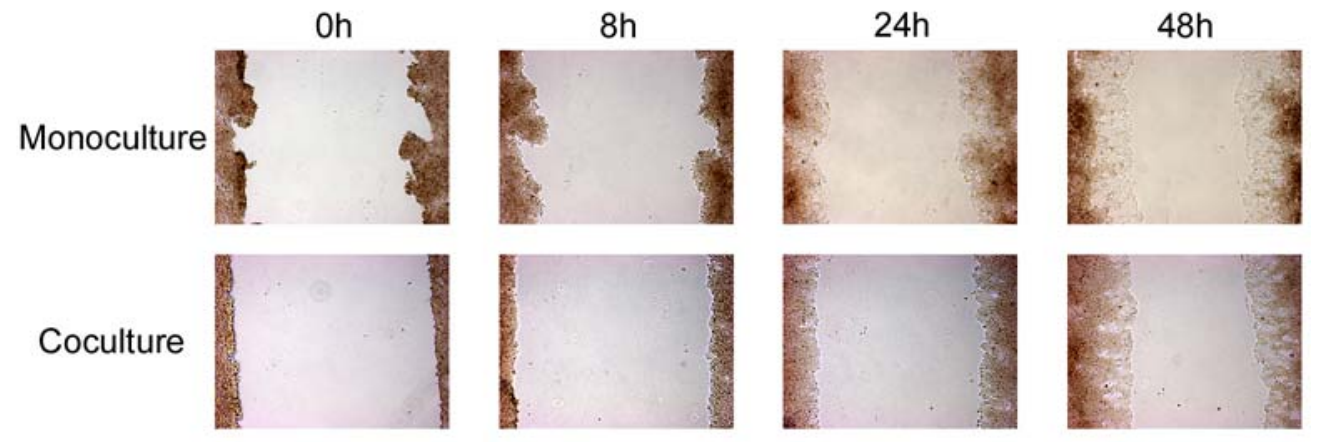

(a) Images of confluent MCF-7 cells after scratching

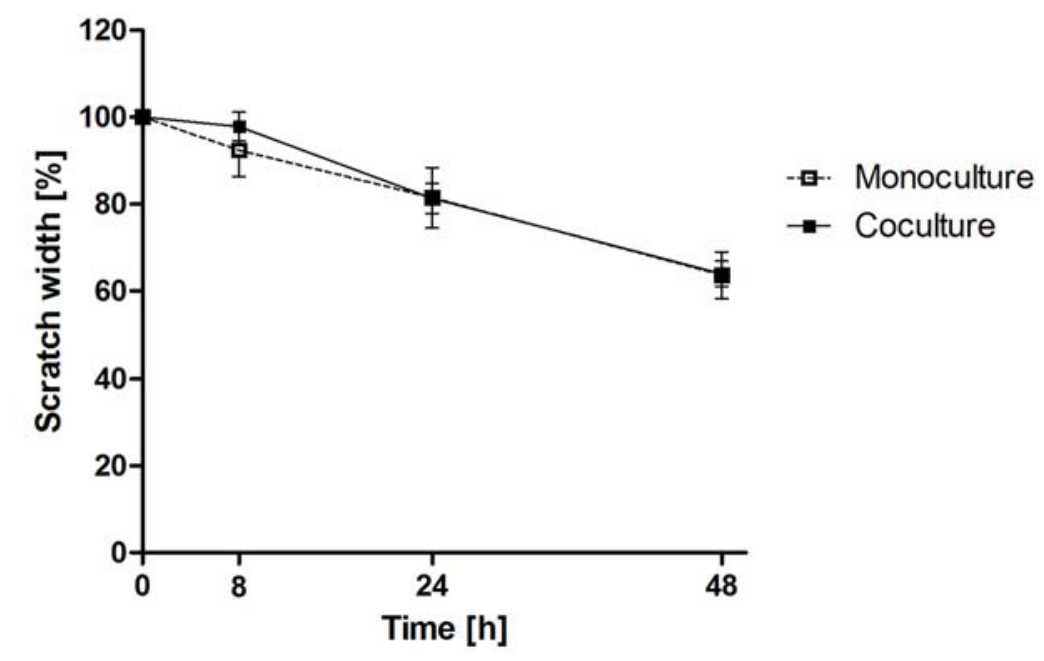

(b) Scratch width

Figure 3.9: Migratory characteristics of MCF-7 cells in coculture

Migration of the breast cancer cell line MCF-7 was studied by a wound healing assay for cells in monoculture and cells cocultivated with osteosarcoma cells. Scratching was performed after cell layers reached confluency. The scratches were studied directly after cell wounding $(0 \mathrm{~h})$ and after $8 \mathrm{~h}, 24 \mathrm{~h}$ and $48 \mathrm{~h}$. (a) Bright field images show the scratches of monocultured (upper line of pictures) and cocultured cells (lower line). (b) Scratch width was measured for monocultured (white rectangles with dotted line) and cocultured cells (black rectangles with continuous line). Data is graphed in relation to the first measurement at $0 \mathrm{~h}$. (White and black rectangles overlie each other in parts in the graph.) Results were analyzed by paired t-test comparing data of monoculture and coculture conditions within one point in time (mean \pm SEM; $n=5)$.

In sum, cocultivation of breast cancer cells with osteosarcoma cells led to different effects depending on the studied cell line. MDA-MB-231 and MDA-MB-435s cells showed an increased invasive behavior up to $48 \mathrm{~h}$. Then, both cell lines invaded stronger in monoculture. In HCC 1806 and MCF-7 cells, invasion in coculture increased continuously over $96 \mathrm{~h}$. In contrast, cell migration in MCF-7 cells was not changed by coculture conditions. 


\subsubsection{Influence of TGF $\beta_{1}$ treatment on breast cancer cell invasion}

$\mathrm{TGF} \beta_{1}$ as one mediator of EMT was studied on breast cancer cell invasion in monocultured and cocultured cells. Invasion was studied in MDA-MB-231, HCC 1806 and MCF-7 cells treated with $2 \mu \mathrm{M} \mathrm{TGF} \beta_{1}$ for $48 \mathrm{~h}$. Results are analyzed related to the invasion in monocultured cells without treatment.

In MDA-MB-231, a significantly increased invasion was observed in cocultured cells under control conditions $(100 \% \pm 15 \%$ SEM vs. $221 \% \pm 37 \%$ SEM; see figure 3.10). Treatment with TGF $\beta_{1}$ showed a trend towards more invasion in monoculture and coculture $(183 \% \pm 74 \%$ SEM vs. $298 \% \pm 42 \%$ SEM). Direct comparison of treated and untreated samples for monoculture respectively coculture showed no significant differences.

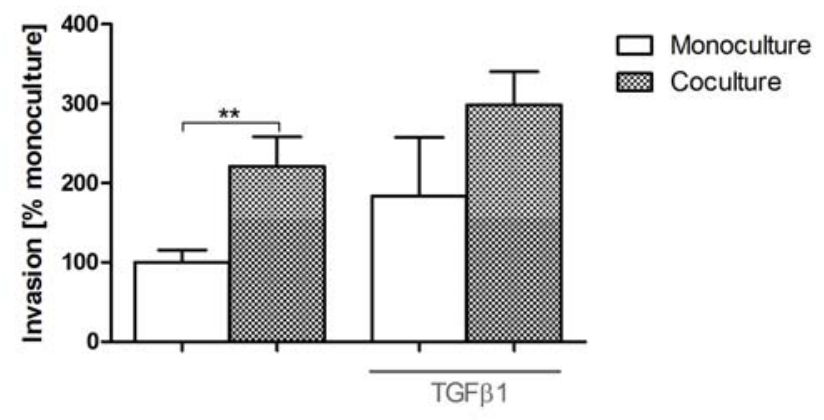

Figure 3.10: Invasion of MDA-MB-231 cells treated with TGF $\beta_{1}$

Invasion of the breast cancer cell line MDA-MB-231 was studied by a modified Boyden chamber assay for cells treated with $\mathrm{TGF} \beta_{1}[2 \mu \mathrm{M}]$ and controls without treatment in monoculture (white columns) and cocultivated with osteosarcoma cells (dotted columns). Invasion is graphed in relation to the invaded cells in monoculture without treatment after $48 \mathrm{~h}$. Results were analyzed by paired t-test comparing data constant in culture conditions respectively treatment (mean $\pm \mathrm{SEM} ; * *: \mathrm{p}<0.01 ; \mathrm{n}=6$ ).

In figure 3.11, HCC 1806 cells invaded significantly stronger in coculture compared to monoculture under control conditions ( $100 \% \pm 16 \%$ SEM vs. $847 \% \pm 252 \%$ SEM). Cells treated with TGF $\beta_{1}$ showed a stronger invasion (510\% $\pm 82 \%$ SEM vs. $910 \% \pm 391 \%$ SEM). The difference in invaded cells is significant for monocultured cells with and without exposition to TGF $\beta_{1}$. 


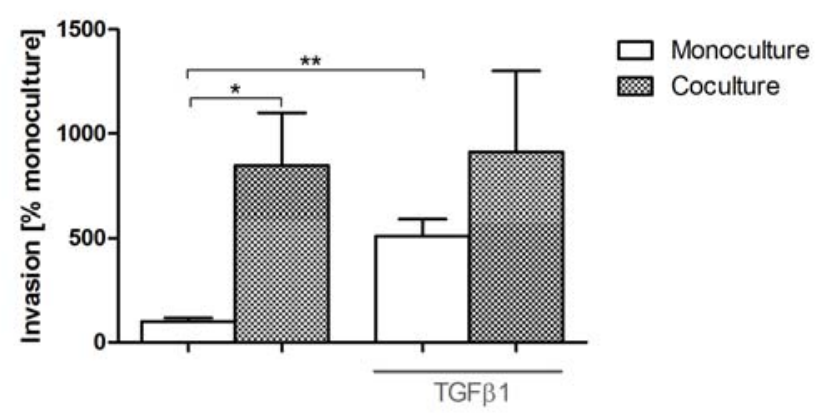

Figure 3.11: Invasion of HCC 1806 cells treated with TGF $\beta_{1}$

Invasion of the breast cancer cell line HCC 1806 was studied by a modified Boyden chamber assay for cells treated with $\mathrm{TGF} \beta_{1}[2 \mu \mathrm{M}]$ and controls without treatment in monoculture (white columns) and cocultivated with osteosarcoma cells (dotted columns). Invasion is graphed in relation to the invaded cells in monoculture without treatment after $48 \mathrm{~h}$. Results were analyzed by paired t-test comparing data constant in culture conditions respectively treatment (mean \pm SEM; *: $\mathrm{p}<0.05 ; * *: \mathrm{p}<0.01 ; \mathrm{n}=7$ ).

Results for MCF-7 cells are graphed in figure 3.12. Invasion of cocultured cells was significantly increased under control conditions (100\% $\pm 20 \%$ SEM vs. $724 \% \pm 188 \%$ SEM). Incubation with TGF $\beta_{1}$ showed little effect on monocultured cells $(76 \% \pm 8 \%$ SEM). Cocultured cells invaded stronger $(1973 \% \pm 693 \%$ SEM) with no significant difference between control conditions and treatment.

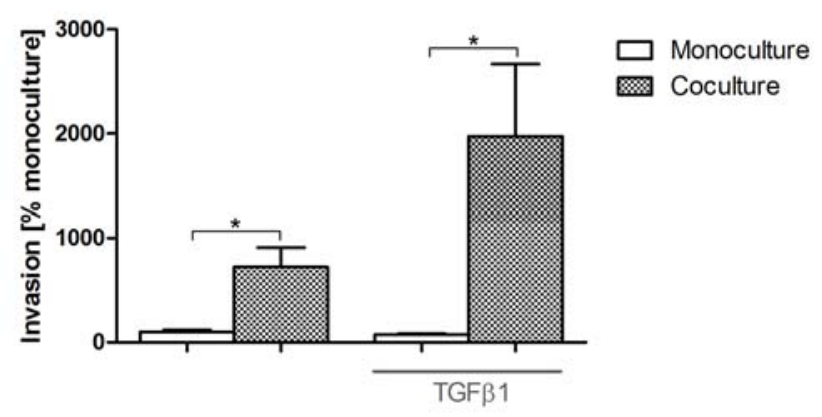

Figure 3.12: Invasion of MCF-7 cells treated with TGF $\beta_{1}$

Invasion of the breast cancer cell line MCF-7 was studied by a modified Boyden chamber assay for cells treated with $\operatorname{TGF} \beta_{1}[2 \mu \mathrm{M}]$ and controls without treatment in monoculture (white columns) and cocultivated with osteosarcoma cells (dotted columns). Invasion is graphed in relation to the invaded cells in monoculture without treatment after $48 \mathrm{~h}$. Results were analyzed by paired t-test comparing data constant in culture conditions respectively treatment (mean \pm SEM; *: $\mathrm{p}<0.05 ; \mathrm{n}=6)$.

Summing up, the effect of $\mathrm{TGF} \beta_{1}$ treatment on invasion was marginal. Only HCC 1806 cells showed stronger invasion by $\mathrm{TGF} \beta_{1}$ in monoculture. No significant difference was observed for the other cell lines for treated and untreated cells within monoculture and respectively coculture. 


\subsubsection{Motility marker expression affected by cocultivation and TGF $\beta_{1}$}

Investigations on motility marker expression were done by mRNA analysis (see chapters 2.2.3.12.2.3.4). The cell lines MDA-MB-231, HCC 1806 and MCF-7 were studied under monoculture or coculture conditions and treatment with $2 \mu \mathrm{M}$ TGF $\beta_{1}$ after $24 \mathrm{~h}$. The same marker were used as in chapter 3.2.4.

In figure 3.13, expression of the epithelial marker is graphed. The results for CDH1 are shown in figure 3.13(a). No difference was observed between monocultured and cocultured cells in all of the studied cell lines (MDA-MB-231: $100 \%$ vs. $94 \% \pm 10 \%$ SEM; HCC 1806: $100 \%$ vs. $101 \% \pm 3 \%$ SEM; MCF-7: $100 \%$ vs. $94 \% \pm 3 \%$ SEM). CDH1 expression was not changed significantly by TGF $\beta_{1}$ treatment in cocultured MDA-MB-231 cells (134\% $\pm 35 \%$ SEM) and HCC 1806 cells ( $95 \% \pm 6 \%$ SEM). Significantly lower expression levels of CDH1 were found in cocultured MCF-7 cells exposed to TGF $\beta_{1}(77 \% \pm 5 \%$ SEM).

Further studies on TJP1 showed different results (see figure 3.13(b)). Little changes in expression levels of TJP1 were observed by comparison of monoculture and coculture conditions (MDA-MB-231: $100 \%$ vs. $113 \% \pm 14 \%$ SEM; HCC 1806: $100 \%$ vs. $101 \% \pm 7 \%$ SEM; MCF-7: $100 \%$ vs. $96 \% \pm 2 \%$ SEM) with no significance. Cocultured cells showed increased expression by TGF $\beta_{1}$ treatment in MDA-MB-231 (145\% $\pm 13 \%$ SEM) and in HCC $1806(130 \% \pm 10 \%$ SEM) with a significant difference. In treated MCF-7 cells, TJP1 expression was significantly decreased $(85 \% \pm 3 \%$ SEM).

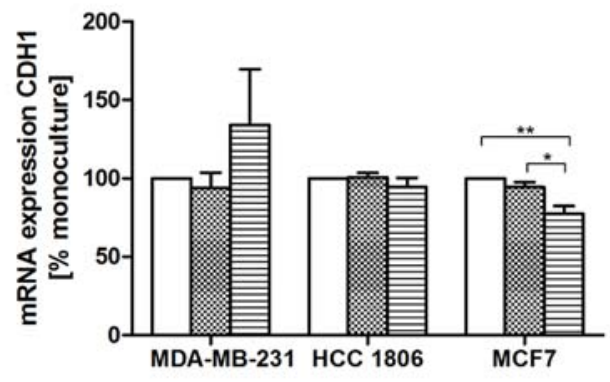

(a) $\mathrm{CDH} 1$

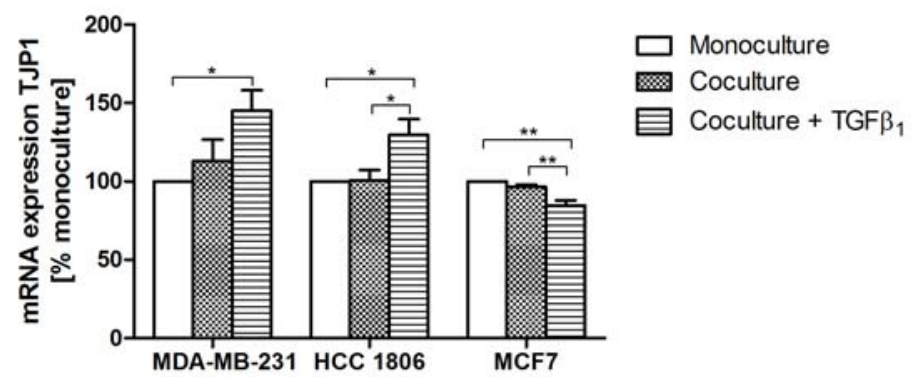

(b) TJP1

Figure 3.13: Expression of epithelial marker in breast cancer cells treated with TGF $\beta_{1}$

Epithelial marker distribution was investigated in the breast cancer cell lines MDA-MB-231, HCC 1806 and MCF-7 by gene expression analysis via RT-PCR. Expression was standardized to the expression of the housekeeping gene L7 for each condition and normalized to the expression in monoculture. Levels of CDH1 (a) and TJP1 (b) expression are graphed for monocultured cells (white columns), cocultured cells (dotted columns) and cocultured cells treated with TGF $\beta_{1}$ (lined columns). Results were analyzed by 1way ANOVA and Tukey's multiple comparison test within one cell line (mean \pm SEM; *: $p<0.05 ; * *: p<0.01 ; n=4$ ). 
Expression of the mesenchymal marker VIM and S100A4 is shown in figure 3.14(a) and (b). Comparing untreated monoculture and coculture gene expression for VIM, no significant changes in expression levels were observed in all of the cell lines (MDA-MB-231: $100 \%$ vs. $94 \% \pm$ $5 \%$ SEM; HCC 1806: $100 \%$ vs. $151 \% \pm 32 \%$ SEM; MCF-7: $100 \%$ vs. $90 \% \pm 6 \%$ SEM). Cocultured cells treated with TGF $\beta_{1}$ had significantly increased expression levels in MDA-MB-231 $(148 \% \pm 7 \%$ SEM) and HCC 1806 cells $(1059 \% \pm 56 \%$ SEM). In MCF-7 cells, VIM expression was significantly decreased $(69 \% \pm 4 \%$ SEM).

Expression analysis for S100A4 showed different changes in cocultured cells compared to monoculture. In MDA-MB-231 cells, S100A4 expression was not altered (100\% vs. $109 \% \pm 7 \%$ SEM). The cell line HCC 1806 had significantly decreased S100A4 expression (100\% vs. $94 \% \pm$ $2 \%$ SEM). MCF-7 cells showed a significantly increased expression by cocultivation (100\% vs. $114 \% \pm 3 \%$ SEM). Treatment with TGF $\beta_{1}$ led to significantly decreased gene expression in all of the cell lines (MDA-MB-231: $87 \% \pm 4 \%$ SEM; HCC 1806: $43 \% \pm 1 \%$ SEM; MCF-7: $88 \%$ $\pm 3 \%$ SEM).

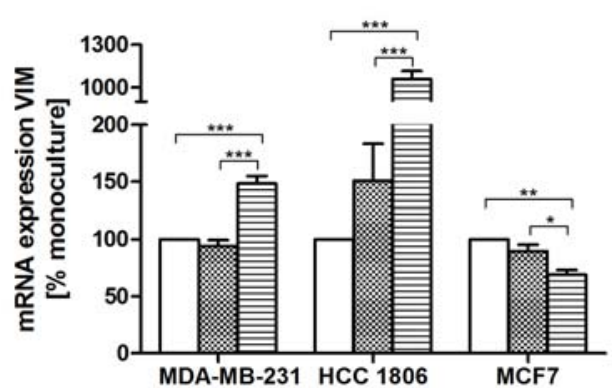

(a) VIM

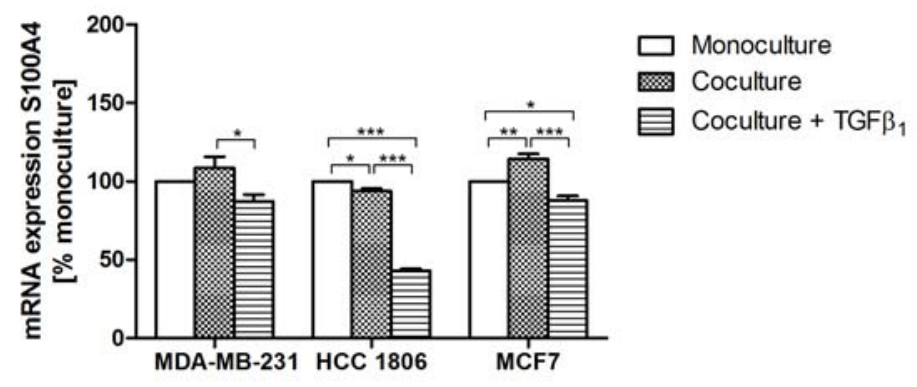

(b) S100A4

Figure 3.14: Expression of mesenchymal marker in breast cancer cells treated with TGF $\beta_{1}$ Mesenchymal marker distribution was investigated in the breast cancer cell lines MDA-MB-231, HCC 1806 and MCF-7 by gene expression analysis via RT-PCR. Expression was standardized to the expression of the housekeeping gene L7 for each condition and normalized to the expression in monoculture. Levels of VIM (a) and S100A4 (b) expression are graphed for monocultured cells (white columns), cocultured cells (dotted columns) and cocultured cells treated with TGF $\beta_{1}$ (lined columns). Results were analyzed by 1way ANOVA and Tukey's multiple comparison test within one cell line (mean \pm SEM; $*: p<0.05$; $* *: p<0.01 ; * *: p<0.001 ; \mathrm{VIM}: \mathrm{n}=4$ in MDA-MB-231, MCF-7; $n=3$ in HCC 1806; S100A4: $n=4)$.

Taken together, no great changes in epithelial and mesenchymal marker expression was observed in MDA-MB-231, HCC 1806 and MCF-7 cells by cocultivation with osteosarcoma cells. Treatment with TGF $\beta_{1}$ affected motility marker expression especially for TJP1, VIM and S100A4. A consistent gene regulation was only seen for S100A4 in all of the tested cell lines. 


\subsection{Gene expression in MCF-7 cells during cocultivation}

MCF-7 cells showed a different invasive behavior during cocultivation with osteosarcoma cells (see chapter 3.2.2.3). Expression analysis of genes involved in EMT revealed no connection between increased invasion and EMT processes (see chapter 3.2.4). A microarray analysis was carried out to get information on the gene pattern regulated by cocultivation.

\subsubsection{Microarray analysis}

mRNA samples of monocultured and cocultured MCF-7 cells were analyzed by a microarray technique (see chapter 2.2.3.1.2 and 2.2.3.5). The data was clustered for the six samples (three independent samples for each condition; see Appendix A1) and showed a correlation within each condition.

The results show that the expression of 325 genes was changed comparing monocultured and cocultured samples (see Appendix A2). Data was $\log _{2}$-transformed. Expression intensity was analyzed as fold change $(F C)$. A change was considered as meaningful, if it was at least two-fold upregulated $(\log F C$ treatment-control $>1$ ) respectively downregulated $(\log F C$ treatment-control $<-1)$. The heatmap in figure 3.15 shows the 50 genes which were regulated the most. The monocultured samples are represented as controls and the cocultured samples are named treatments. The expression of these genes was at least up to $2.14 \operatorname{logFC}$ respectively not exceeding $-2.17 \log F C$. A black area shows no difference, a blue area shows less expression and a yellow area shows upregulated expression of the treatment group compared to the control group. 44 genes (88\%) were upregulated and six genes $(12 \%)$ were downregulated. 


\section{Color Key}

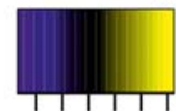

\section{Heatmap Treatment-Control}

$\begin{array}{lll}-1 & 0 & 1\end{array}$

Row Z-Score

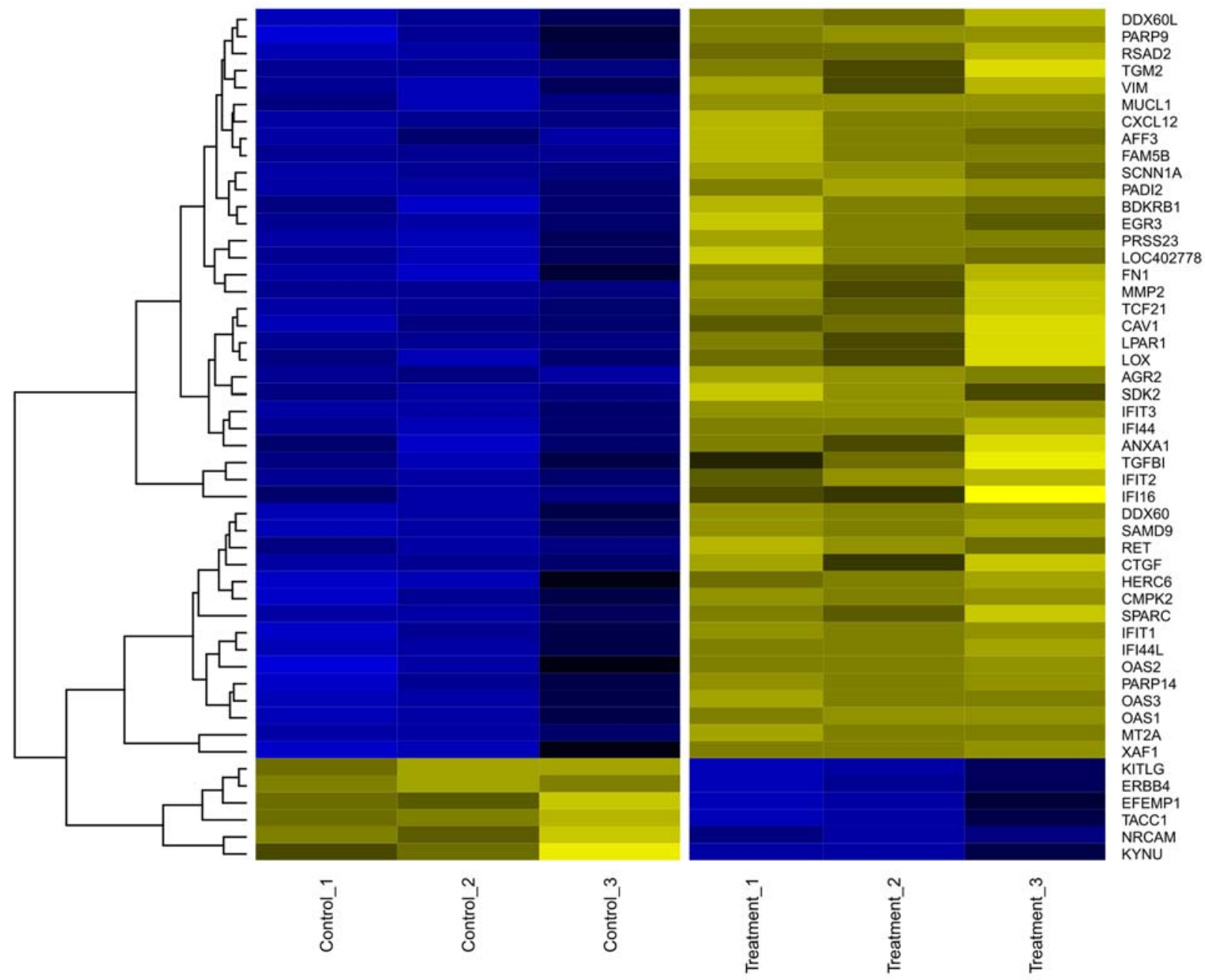

Figure 3.15: Heatmap of genes most affected by cocultivation

The breast cancer cell line MCF-7 was studied by microarray analysis for genes which were regulated during cocultivation with osteosarcoma cells. Three independent samples of monocultured cells (controls) and of cocultured cells (treatments) were investigated. The heatmap shows the 50 genes which were regulated the most (black area $=$ no difference between control and treatment; blue area $=$ downregulated expression; yellow area $=$ upregulated expression). Data was $\log _{2}$-transformed. Expression intensity is represented as fold change (FC).

The 50 genes were classified in five groups (see table 3.1). The first group represents genes involved in immune response activities including inflammation, viral infection and interferon dependent processes. The second group is characterized by mechanisms covering cell motility, adhesion, metastasis and cell morphology. Genes involved in cancer, oncogenes and genes affected by oncogenes are grouped in the third column. The fourth group of genes participate in proliferation 
processes. Genes with no reference to the mentioned groups are taken together as others in the last column.

\begin{tabular}{|c|c|c|c|c|}
\hline Immune response & Cell motility & Cancer & Proliferation & Others \\
\hline \hline IFIT2 & TGFBI & CTGF & KITLG & CMPK2 \\
\hline BDKRB1 & SDK2 & ERBB4 & ERBB4 & PARP14 \\
\hline MMP2 & MMP2 & AFF3 & MMP2 & PRSS23 \\
\hline MT2A & SAMD9 & MT2A & SAMD9 & DDX60L \\
\hline EGR3 & EGR3 & EGR3 & EGR3 & HERC6 \\
\hline IFI16 & MUCL1 & TGM2 & IFI16 & LOC402778 \\
\hline OAS1 & CAV1 & CAV1 & & PADI2 \\
\hline RSAD2 & AGR2 & XAF1 & & FAM5B \\
\hline DDX60 & LPAR1 & LPAR1 & & SCNN1A \\
\hline IFIT1 & EFEMP1 & EFEMP1 & & \\
\hline ANXA1 & RET & RET & & \\
\hline OAS3 & LOX & & & \\
\hline PARP9 & PARP9 & & & \\
\hline VIM & VIM & & & \\
\hline IFIT3 & FN1 & & & \\
\hline CXCL12 & CXCL12 & & & \\
\hline OAS2 & TCF21 & & & \\
\hline IFI44 & NRCAM & & & \\
\hline IFI44L & TACC1 & & & \\
\hline SPARC & SPARC & & & \\
\hline KYNU & & & & \\
\hline
\end{tabular}

Table 3.1: Classification of genes most affected by cocultivation

The 50 genes were classified with respect to their known cellular functions. Some genes were allocated to more than one group. Each column is representative for processes and mechanisms involved in the function mentioned in the headline (immune response: inflammation, viral infection, interferon dependent processes; cell motility: adhesion, metastasis, cell morphology; cancer: oncogenes, genes affected by oncogenes; proliferation: cell growth).

The relative distribution of the 50 genes was as follows: $42 \%$ of the genes are involved in immune response processes, $40 \%$ of the genes are active in cell motility processes, $22 \%$ of the genes participate in cancer processes, $12 \%$ of the genes are involved in cell proliferation and $18 \%$ of the genes show no relation to these four groups. Because of multiple entry of one gene to more than one group, the sum of the ratios exceeds $100 \%$.

\subsubsection{Expression of genes involved in cell motility processes}

The results of the microarray were validated by qPCR for selected genes (see chapter 2.2.3.7.1). Eight genes involved in cell motility processes were chosen. Transforming acidic coiled-coil containing protein 1 (TACC1) as one gene downregulated by coculture conditions and VIM, CXCL12, 
fibronectin 1 (FN1), poly (ADP-ribose) polymerase 9 (PARP9), MMP2, secreted protein acidic and rich in cysteine (SPARC) and transcription factor 21 (TCF21) as genes, which were upregulated by the treatment, were analyzed.

In figure 3.16, the results of the qPCR analysis are graphed. The observed downregulation of TACC1 was approved by significant data $(0.14 \% \pm 0.01 \%$ SEM). FN1 showed a significant higher expression in cocultured cells $(6.43 \% \pm 1.03 \%$ SEM). A trend towards an upregulated expression of VIM (55.14\% $\pm 44.92 \%$ SEM), CXCL12 (7.37\% $\pm 2.01 \%$ SEM), PARP9 (4.56 \% $\pm 1.00 \%$ SEM), MMP2 $(62.65 \% \pm 29.74 \%$ SEM $)$, SPARC $(61.59 \% \pm 16.10 \%$ SEM $)$ and TCF21 $(11.01 \% \pm 2.51 \%$ SEM) was observed with no significant results.

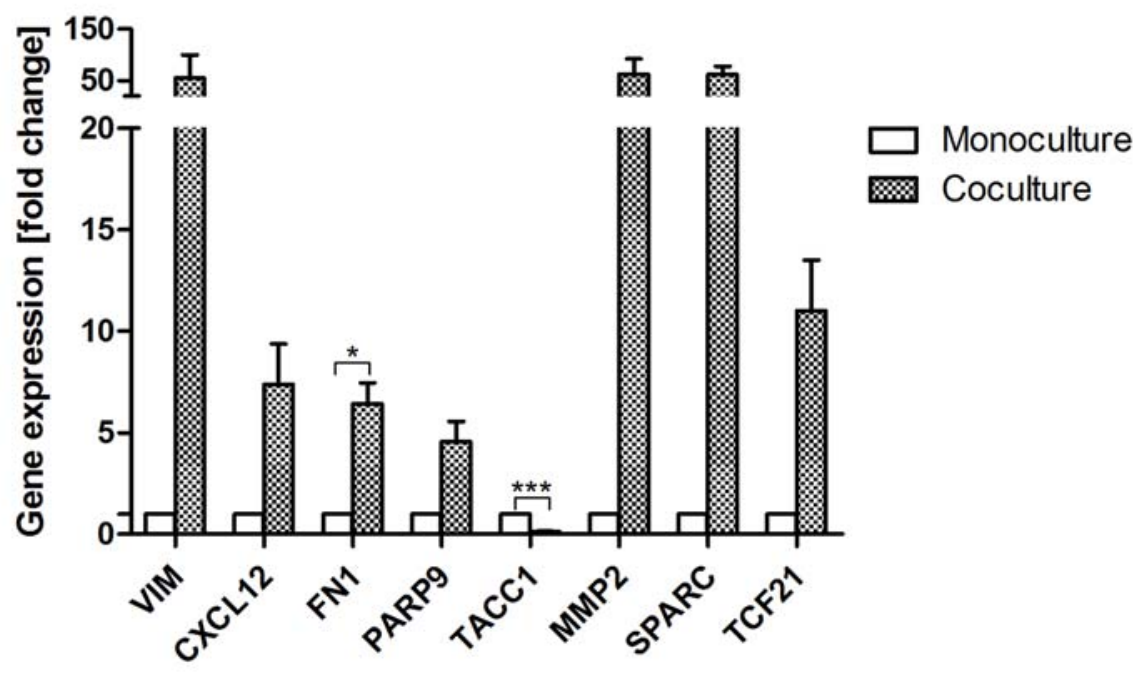

Figure 3.16: Gene expression in breast cancer cells affected by cocultivation

Gene expression of selected genes was investigated in the breast cancer cell line MCF-7 by qPCR. Expression was standardized to the expression of the housekeeping gene YWHAZ and normalized to the expression in monoculture. Levels of gene expression are graphed for monocultured cells (white columns) and cocultured cells (dotted columns). Results were analyzed by paired t-test comparing data of monoculture and coculture conditions for one gene (mean \pm SEM; *: $\mathrm{p}<0.05 ; * * *: \mathrm{p}<0.001 ; \mathrm{n}=3$ ).

In summary, microarray analysis of cocultivated MCF-7 cells showed changes in expression levels of genes involved mostly in immune response, cell motility, cancer and proliferation processes. Verification of the data by $\mathrm{qPCR}$ for selected genes validated the microarray data.

\subsection{Effects of kisspeptin-10 and analogs on bone-directed invasion}

The inhibitory effect of KP-10 was shown in the breast cancer cell lines MCF-7 and MDA-MB-231 [Olbrich, 2010a, Olbrich et al., 2010b]. KP-10 inhibited cell invasion within a concentration 
window. MCF-7 cells were inhibited significantly by $10^{-9} \mathrm{M}$ up to $10^{-11} \mathrm{M}$ of KP-10, whereas MDA-MB-231 cells showed significantly less invasion by $10^{-10} \mathrm{M}$ KP-10. The experiments were continued for the cell line HCC 1806 based on the experimental setting in chapter 2.2.2.1. Further invasion studies for the KP-10 analog DK6-kisspeptin-10 (DKP-10) were carried out. DKP-10 was revealed by substitution of Phe at position six with dLys.

\subsubsection{Invasion of breast cancer cells treated with kisspeptin-10}

Invasion of HCC 1806 cells was measured after $72 \mathrm{~h}$ and daily treatment with KP-10 in different concentrations.

Results of the coculture controls are represented in figure 3.17(a). Invasion of cells in coculture was significantly increased $(100 \% \pm 8 \%$ SEM vs. $2066 \% \pm 306 \%$ SEM). Treatment with KP-10 in concentrations of $10^{-14} \mathrm{M}$ to $10^{-8} \mathrm{M}$ showed no effect on monocultured cells (data not shown). No significant inhibition was observed in coculture by KP-10 (coculture: $100 \% \pm$ $5 \%$ SEM; $10^{-14} \mathrm{M}: 58 \% \pm 7 \%$ SEM; $10^{-13} \mathrm{M}: 90 \% \pm 18 \%$ SEM; $10^{-12} \mathrm{M}: 72 \% \pm 9 \%$ SEM; $10^{-11} \mathrm{M}: 102 \% \pm 22 \%$ SEM; $10^{-10} \mathrm{M}: 88 \% \pm 13 \%$ SEM; $10^{-9} \mathrm{M}: 63 \% \pm 7 \%$ SEM; $10^{-8} \mathrm{M}$ : $98 \% \pm 7 \%$ SEM; see figure $3.17(\mathrm{~b}))$.

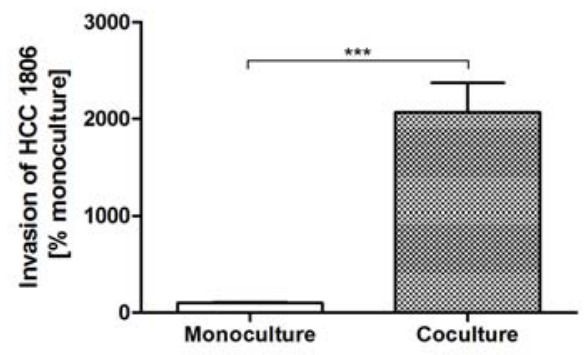

(a) Invasion of HCC 1806

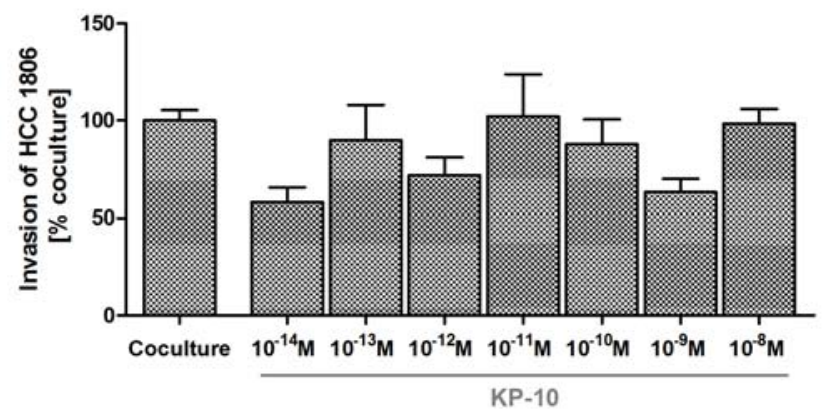

(b) Invasion of cocultured cells treated with KP-10

Figure 3.17: Invasion of HCC 1806 cells treated with KP-10

Invasion was studied by a modified Boyden chamber assay for the breast cancer cell line HCC 1806 treated with KP-10. (a) Invasion of HCC 1806 cells was measured without treatment in monoculture (white columns) and cocultivated with osteosarcoma cells (dotted columns). Invasion is graphed in relation to the invaded cells in monoculture after $72 \mathrm{~h}$. Results were analyzed by paired t-test (mean $\pm \mathrm{SEM} ; * * *: p<0.001 ; n=20$ ). (b) Invasion of cocultured HCC 1806 cells was analyzed under treatment with KP-10 daily in different concentrations. Invasion is graphed in relation to the invaded cells in coculture without treatment after $72 \mathrm{~h}$. Results were analyzed by 1way ANOVA and Dunnett's multiple comparison test (mean $\pm S E M ; n=20$ for coculture control; $n=8$ for $\mathrm{KP}-10\left[10^{-14} \mathrm{M}, 10^{-13} \mathrm{M}\right] ; \mathrm{n}=12$ for $\mathrm{KP}-10\left[10^{-12} \mathrm{M}\right.$ to $\left.\left.10^{-8} \mathrm{M}\right]\right)$. 


\subsubsection{Invasion of breast cancer cells treated with a kisspeptin-10 analog}

Invasion of HCC 1806 cells was measured after $72 \mathrm{~h}$ and daily treatment with DKP-10 in different concentrations.

Invasion in coculture was significantly increased without treatment (100\% $18 \%$ SEM vs. $2219 \%$ $\pm 386 \%$ SEM; see figure 3.18(a)). Treatment with DKP-10 in concentrations of $10^{-14} \mathrm{M}$ to $10^{-8} \mathrm{M}$ showed no effect on monocultured cells (data not shown). In figure 3.18(b), the effect on invasion by DKP-10 in coculture is graphed. In concentrations of $10^{-12} \mathrm{M}(48 \% \pm 10 \%$ SEM), $10^{-10} \mathrm{M}(61 \% \pm 10 \% \mathrm{SEM})$ and $10^{-9} \mathrm{M}(56 \% \pm 6 \% \mathrm{SEM})$, invasion was decreased significantly. Except the samples treated with $10^{-8} \mathrm{M}$ DKP-10, other concentrations tended to inhibit invasion of cells (coculture: $100 \% \pm 5 \%$ SEM; $10^{-14} \mathrm{M}: 74 \% \pm 11 \% \mathrm{SEM} ; 1^{-13} \mathrm{M}$ : $69 \% \pm 10 \%$ SEM; $10^{-11} \mathrm{M}: 70 \% \pm 17 \%$ SEM; $10^{-8} \mathrm{M}: 101 \% \pm 18 \%$ SEM).

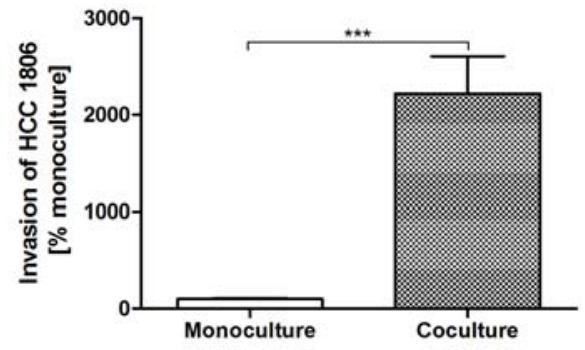

(a) Invasion of HCC 1806

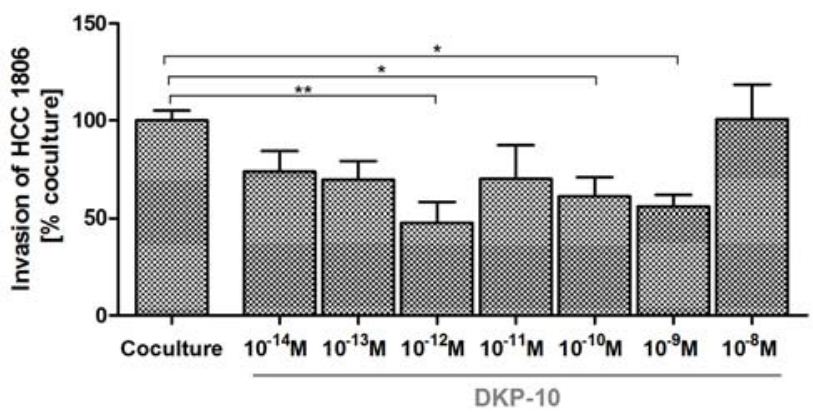

(b) Invasion of cocultured cells treated with DKP-10

Figure 3.18: Invasion of HCC 1806 cells treated with DKP-10

Invasion was studied by a modified Boyden chamber assay for the breast cancer cell line HCC 1806 treated with DKP-10. (a) Invasion of HCC 1806 cells was measured without treatment in monoculture (white columns) and cocultivated with osteosarcoma cells (dotted columns). Invasion is graphed in relation to the invaded cells in monoculture after $72 \mathrm{~h}$. Results were analyzed by paired t-test (mean $\pm S E M$; $* * *: p<0.001 ; n=14$ ). (b) Invasion of cocultured HCC 1806 cells was analyzed under treatment with DKP-10 daily in different concentrations. Invasion is graphed in relation to the invaded cells in coculture without treatment after $72 \mathrm{~h}$. Results were analyzed by 1 way ANOVA and Dunnett's multiple comparison test (mean $\pm \mathrm{SEM} ; *: \mathrm{p}<0.05 ; * *: \mathrm{p}<0.01 ; \mathrm{n}=14$ for coculture control; $n=8$ for KP-10 $\left[10^{-14} \mathrm{M}, 10^{-13} \mathrm{M}\right] ; \mathrm{n}=6$ for $\mathrm{KP}-10\left[10^{-12} \mathrm{M}\right.$ to $\left.10^{-8} \mathrm{M}\right]$ ).

Summing up, KP-10 did not affect HCC 1806 cells cocultivated with osteosarcoma cells significantly. In contrast, the KP-10 analog DKP-10 led to significantly reduced invasion. Both peptides showed no effect on monocultured cells. 


\subsection{Effects of kisspeptin-10 in vivo}

According to the inhibited invasion of breast cancer cells by KP-10 in vitro [Olbrich, 2010a, Olbrich et al., 2010b], the results of the following chapter describe the effect of kisspeptin-10 on metastasis and tumor growth in vivo. Different breast cancer xenograft models in mice were used (see chapter 2.2.6). Metastasis was analyzed in murine organs, as lung, bone and liver. Circulating tumor cells were identified in blood. Tumor growth was measured.

\subsubsection{MDA-MB-231 breast cancer xenograft}

Tumor cell metastasis was studied by intracardiacally injection of MDA-MB-231 cells. Metastasis of CTCs was investigated. Three experimental groups were kept: the control group without tumor cell injection and without treatment, the tumor group with tumor cell injection and without treatment and the treatment group with tumor cell injection and treatment with $\mathrm{KP}-10$ [50 $\mathrm{\mu g} / \mathrm{mouse}]$ daily starting the day after injection. The study lasted up to 34 days.

\subsubsection{Metastasis}

Analysis of the results showed no significant amounts of hDNA in murine organs, lung, liver and bone, and in blood for treated and untreated mice (data not shown).

\subsubsection{HCC 1806 breast cancer xenograft}

Metastasis was studied by injecting HCC 1806 cells orthotopically at the mammary glands of mice. Two tumors were set per mouse. Three experimental groups were kept: the control group without tumor cell injection and without treatment, the tumor group with tumor cell injection and without treatment and the treatment group with tumor cell injection and treatment with KP-10 [50 $\mathrm{\mu g} /$ mouse] daily starting the day after injection. On day 28 , the experiment was determined.

\subsubsection{Tumor growth}

All of the mice in the tumor group and in the treatment group developed tumors at the mammary glands.

The graph in figure 3.19 shows a constant tumor growth in both groups. The tumors in the treatment group grew significantly slower than in the tumor group (day 11: $47.2 \mathrm{~mm}^{3} \pm 13.9 \mathrm{~mm}^{3}$ SEM vs. $105.3 \mathrm{~mm}^{3} \pm 10.7 \mathrm{~mm}^{3} \mathrm{SEM}$; day $14: 79.2 \mathrm{~mm}^{3} \pm 16.5 \mathrm{~mm}^{3} \mathrm{SEM}$ vs. $133.2 \mathrm{~mm}^{3} \pm$ $13.1 \mathrm{~mm}^{3}$ SEM; day $17: 134.7 \mathrm{~mm}^{3} \pm 25.0 \mathrm{~mm}^{3}$ SEM vs. $239.4 \mathrm{~mm}^{3} \pm 22.5 \mathrm{~mm}^{3}$ SEM; day 20 : 
$209.1 \mathrm{~mm}^{3} \pm 40.9 \mathrm{~mm}^{3}$ SEM vs. $415.3 \mathrm{~mm}^{3} \pm 34.8 \mathrm{~mm}^{3} \mathrm{SEM}$; day $24: 296.8 \mathrm{~mm}^{3} \pm 45.4 \mathrm{~mm}^{3}$ SEM vs. $545.9 \mathrm{~mm}^{3} \pm 45.3 \mathrm{~mm}^{3}$ SEM; day $28: 446.2 \mathrm{~mm}^{3} \pm 58.6 \mathrm{~mm}^{3}$ SEM vs. $674.9 \mathrm{~mm}^{3} \pm$ $\left.71.3 \mathrm{~mm}^{3} \mathrm{SEM}\right)$.

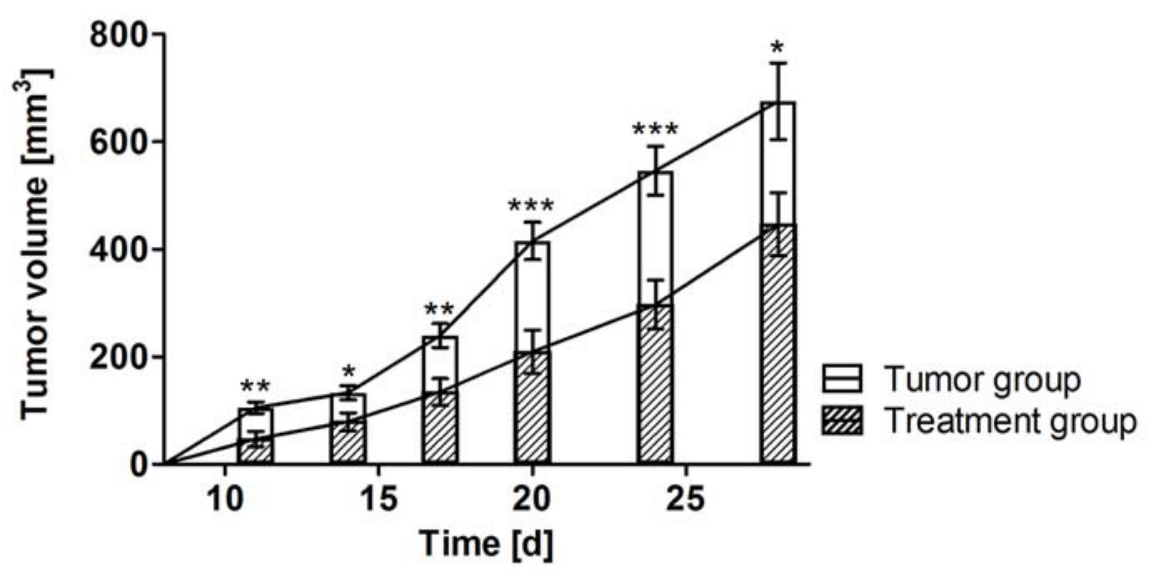

Figure 3.19: Tumor growth in xenografts with HCC 1806

Mice were injected orthotopically at the mammary glands with HCC 1806 breast cancer cells. Two tumors were set per mouse. In the tumor group (white columns), tumor growth was observed without treatment. In the treatment group (shaded columns), treatment with KP-10 [50 $\mathrm{gg} /$ mouse] daily started the day after injection. Tumor growth is represented as tumor volume. Results were analyzed by paired t-test comparing data of tumor and treatment group within one day of measurement (mean $\pm \mathrm{SEM}$; * $\mathrm{p}<0.05$; **: $\mathrm{p}<0.01 ; * *: \mathrm{p}<0.001 ; \mathrm{n}=20$ (ten mice with two tumors/mouse)).

\subsubsection{Metastasis}

hDNA was detected in lung. CTCs were measured in blood. No hDNA was found in bone and liver (data not shown).

In figure 3.20, the amount of hDNA in lung is represented. The results of the tumor group show a significant pattern of hDNA related to the control group (control group: $1.35^{*} 10^{-7} \%$ $\pm 1.35^{*} 10^{-7} \%$ SEM; tumor group: $1.26^{*} 10^{-4} \% \pm 3.63^{*} 10^{-5} \% \mathrm{SEM}$; see figure $\left.3.20 \mathrm{a}\right)$. In the tumor group respectively in the treatment group, eight of ten mice developed metastasis in lung. Comparing the ratio of hDNA in the tumor group and the treatment group, no significant difference was measured (treatment group: $1.80^{*} 10^{-4} \% \pm 1.18^{*} 10^{-4} \%$ SEM; see figure 3.20b). 


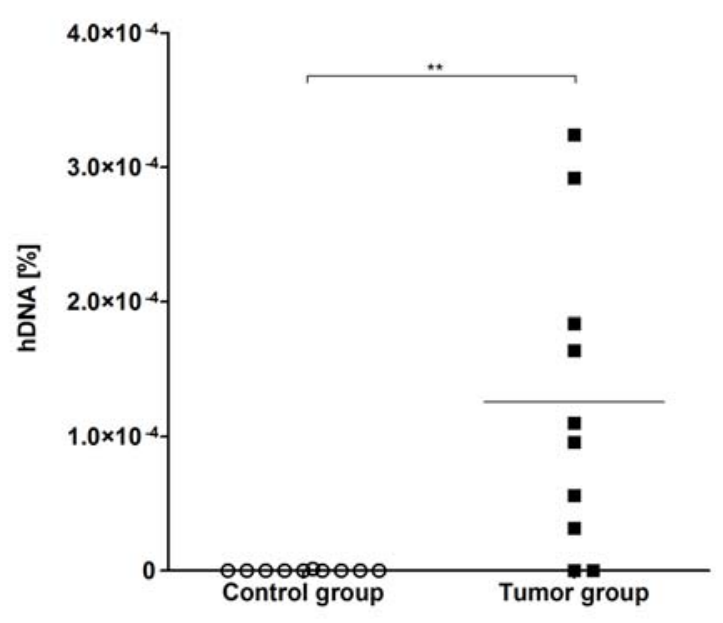

(a) Lung metastasis in untreated mice

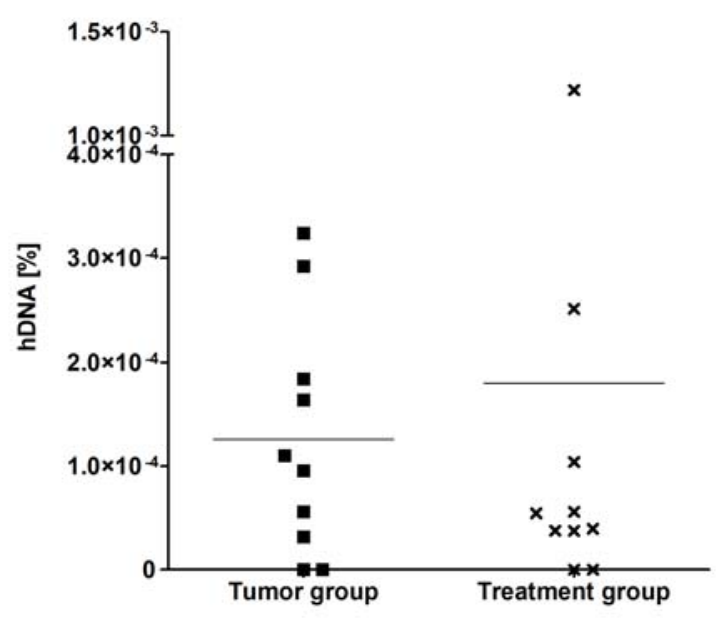

(b) Lung metastasis in mice treated with KP-10

Figure 3.20: Lung metastasis in xenografts with HCC 1806

Mice were injected orthotopically at the mammary glands with HCC 1806 breast cancer cells and treated with $\mathrm{KP}-10[50 \mu \mathrm{g} / \mathrm{mouse}]$ daily starting the day after injection. Lungs were collected at the end of the study and analyzed by DNA isolation and qPCR. Metastasis is represented as ratio of hDNA in mDNA. (a) For verification of the metastasis model, a control group was kept in parallel without tumors and treatment (white circles). In the tumor group (black rectangles), mice were untreated. Results were analyzed by Mann Whitney test (mean; **: $\mathrm{p}<0.01 ; \mathrm{n}=10$ ). (b) Metastasis in the tumor group (black rectangles) and in the treatment group (black crosses) was compared. Results were analyzed by paired t-test (mean; $n=10$ ).

The amount of hDNA detected in blood is graphed in figure 3.21. In seven of ten mice of the tumor group, CTCs were found. Significant more hDNA was measured in contrast to the control group (control group: $1.36^{*} 10^{-6} \% \pm 1.36^{*} 10^{-6} \% \mathrm{SEM}$; tumor group: $7.81^{*} 10^{-4} \% \pm$ $7.41^{*} 10^{-4} \%$ SEM; see figure 3.21a). hDNA was detected in one of the mice of the control group. In the treatment group, in eight of ten mice, tumor cells were circulating in blood. The analysis of hDNA of the treatment group showed comparable results as detected in the tumor group (treatment group: $6.86 * 10^{-4} \% \pm 2.44 * 10^{-4} \% \mathrm{SEM}$; see figure $3.21 \mathrm{~b}$ ). 


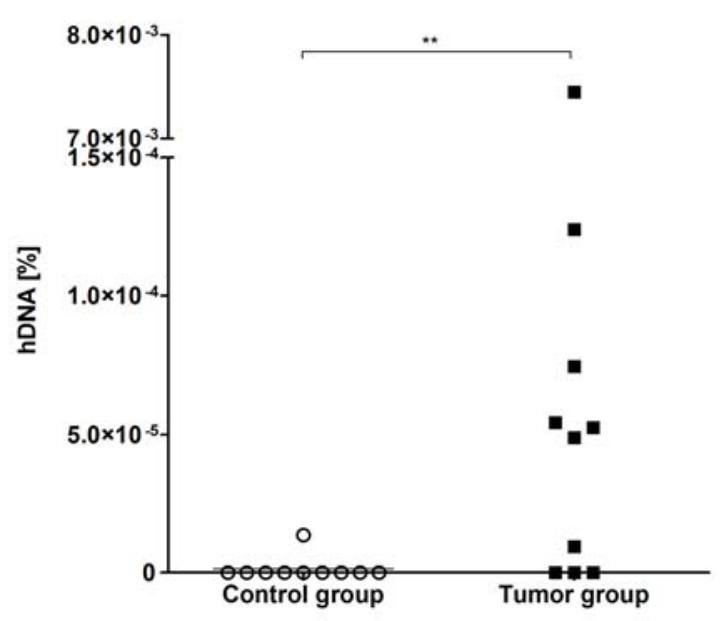

(a) Circulating tumor cells in untreated mice

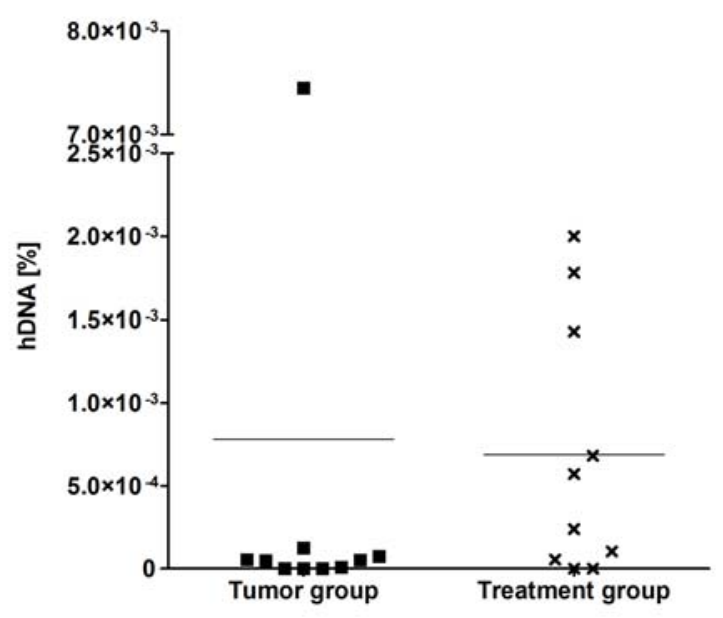

(b) Circulating tumor cells in mice treated with KP-10

Figure 3.21: Circulating tumor cells in xenografts with HCC 1806

Mice were injected orthotopically at the mammary glands with HCC 1806 breast cancer cells and treated with $\mathrm{KP}-10[50 \mu \mathrm{g} /$ mouse] daily starting the day after injection. Blood was collected at the end of the study and analyzed by DNA isolation and GPCR. CTCs are represented as ratio of hDNA in mDNA. (a) For verification of the experimental model, a control group was kept in parallel without tumors and treatment (white circles). In the tumor group (black rectangles), mice were untreated. Results were analyzed by Mann Whitney test (mean; **: $\mathrm{p}<0.01 ; \mathrm{n}=10$ ). (b) CTCs in the tumor group (black rectangles) and in the treatment group (black crosses) were compared. Results were analyzed by paired t-test (mean; $n=10$ ).

\subsubsection{MDA-MB-435s breast cancer xenograft}

MDA-MD-435s cells were injected orthotopically at the mammary glands of mice. Two tumors were set per mouse. Three experimental groups were kept: the control group without tumor cell injection and without treatment, the tumor group with tumor cell injection and without treatment and the treatment group with tumor cell injection and treatment with KP-10 [50 $\mu \mathrm{g} / \mathrm{mouse}]$ daily starting the day after injection. Study was determined on day 41 .

\subsubsection{Tumor growth}

In the tumor group and in the treatment group, $20 \%$ of the injection sites in mice developed no tumors.

In figure 3.22, tumor growth of the tumor group and the treatment group is graphed. Tumors of mice treated with KP-10 showed a trend towards a slower growth rate than untreated mice. The difference was not significant (day 15: $8.8 \mathrm{~mm}^{3} \pm 4.8 \mathrm{~mm}^{3} \mathrm{SEM}$ vs. $17.2 \mathrm{~mm}^{3} \pm 6.7 \mathrm{~mm}^{3}$ SEM; day 18: $22.7 \mathrm{~mm}^{3} \pm 10.4 \mathrm{~mm}^{3}$ SEM vs. $36.9 \mathrm{~mm}^{3} \pm 11.3 \mathrm{~mm}^{3} \mathrm{SEM}$; day 22: $42.2 \mathrm{~mm}^{3}$ $\pm 17.7 \mathrm{~mm}^{3}$ SEM vs. $88.4 \mathrm{~mm}^{3} \pm 27.3 \mathrm{~mm}^{3} \mathrm{SEM}$; day $25: 76.1 \mathrm{~mm}^{3} \pm 28.9 \mathrm{~mm}^{3} \mathrm{SEM}$ vs. 
$129.6 \mathrm{~mm}^{3} \pm 34.9 \mathrm{~mm}^{3}$ SEM; day 29: $112.2 \mathrm{~mm}^{3} \pm 41.7 \mathrm{~mm}^{3}$ SEM vs. $198.8 \mathrm{~mm}^{3} \pm 51.2 \mathrm{~mm}^{3}$ SEM; day 32: $197.3 \mathrm{~mm}^{3} \pm 69.0 \mathrm{~mm}^{3}$ SEM vs. $289.6 \mathrm{~mm}^{3} \pm 71.3 \mathrm{~mm}^{3} \mathrm{SEM}$; day $36: 281.6 \mathrm{~mm}^{3}$ $\pm 88.7 \mathrm{~mm}^{3}$ SEM vs. $407.6 \mathrm{~mm}^{3} \pm 93.3 \mathrm{~mm}^{3} \mathrm{SEM}$; day $39: 394.8 \mathrm{~mm}^{3} \pm 116.4 \mathrm{~mm}^{3} \mathrm{SEM}$ vs. $507.0 \mathrm{~mm}^{3} \pm 120.2 \mathrm{~mm}^{3}$ SEM; day $41: 459.0 \mathrm{~mm}^{3} \pm 135.7 \mathrm{~mm}^{3}$ SEM vs. $574.5 \mathrm{~mm}^{3} \pm$ $\left.134.6 \mathrm{~mm}^{3} \mathrm{SEM}\right)$.

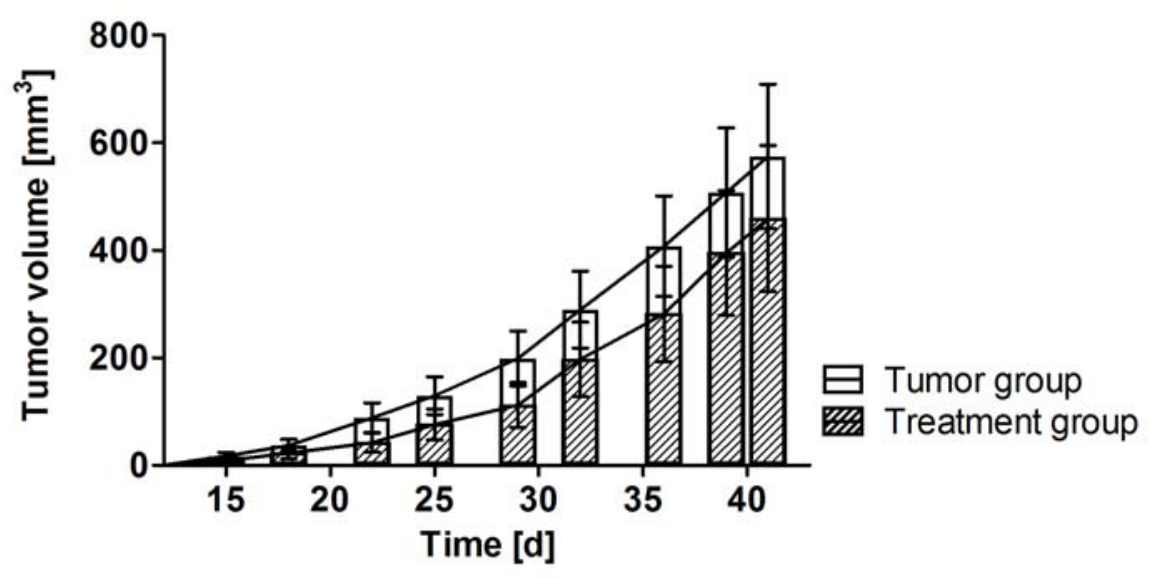

Figure 3.22: Tumor growth in xenografts with MDA-MB-435s

Mice were injected orthotopically at the mammary glands with MDA-MB-435s breast cancer cells. Two tumors were set per mouse. In the tumor group (white columns), tumor growth was observed without treatment. In the treatment group (shaded columns), treatment with KP-10 [50 $\mu \mathrm{g} /$ mouse] daily started the day after injection. Tumor growth is represented as tumor volume. Results were analyzed by paired t-test comparing data of tumor and treatment group within one day of measurement (mean $\pm \mathrm{SEM} ; \mathrm{n}=20$ (ten mice with two tumors/mouse)).

\subsubsection{Metastasis}

hDNA was detected in lung, but not in bone (data not shown).

The graph in figure 3.23 represents the ratio of hDNA in lung. In figure 3.23a, the results are graphed for the control group and the tumor group. In the tumor group, in eight of ten mice, the cells metastasized to lung. hDNA was found in one of the mice of the control group. The amount of hDNA was significantly different (control group: $3.40 * 10^{-5} \% \pm 3.29 * 10^{-5} \%$ SEM; tumor group: $1.08 * 10^{-3} \% \pm 9.08 * 10^{-4} \% \mathrm{SEM}$ ). Figure $3.23 \mathrm{~b}$ shows the ratio of hDNA for the tumor group and the treatment group, which showed metastasis in seven of ten mice. A trend towards lower hDNA amounts in the treated mice was observed with no significance (treatment group: $2.19 * 10^{-4} \% \pm 1.35 * 10^{-4} \% \mathrm{SEM}$ ). 


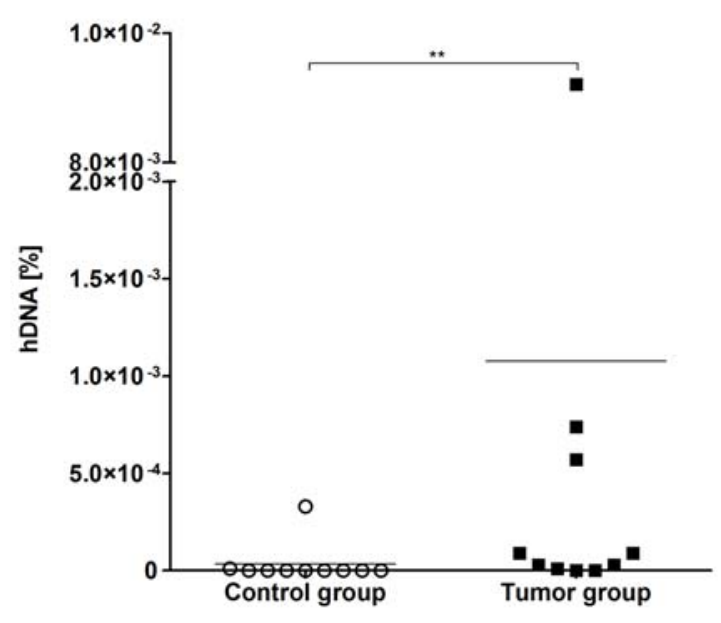

(a) Lung metastasis in untreated mice

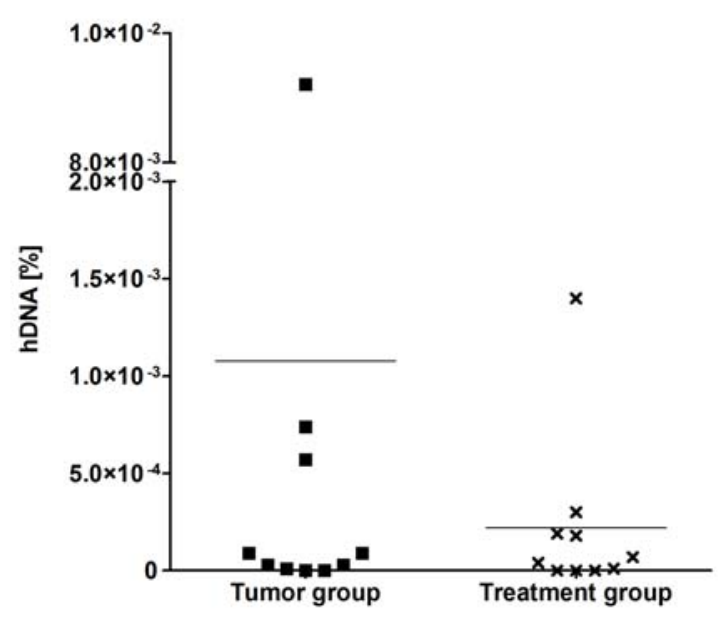

(b) Lung metastasis in mice treated with KP-10

Figure 3.23: Lung metastasis in xenografts with MDA-MB-435s

Mice were injected orthotopically at the mammary glands with MDA-MB-435s breast cancer cells and treated with $\mathrm{KP}-10$ [50 $\mu \mathrm{g} /$ mouse] daily starting the day after injection. Lungs were collected at the end of the study and analyzed by DNA isolation and GPCR. Metastasis is represented as ratio of hDNA in mDNA. (a) For verification of the metastasis model, a control group was kept in parallel without tumors and treatment (white circles). In the tumor group (black rectangles), mice were untreated. Results were analyzed by Mann Whitney test (mean; $* *: \mathrm{p}<0.01 ; \mathrm{n}=10$ ). (b) Metastasis in the tumor group (black rectangles) and in the treatment group (black crosses) was compared. Results were analyzed by paired t-test (mean; $n=10$ ).

In summary, two xenograft models were studied for metastasis. Intracardiac injection of MDA-MB-231 cells did not lead to detectable metastasis. After orthotopical injection of HCC 1806 and MDA-MB-435s cells, tumors developed. Sporadic metastasis was observed in lung and blood for some of the mice of the experimental groups. An effect of KP-10 treatment on metastasis in vivo could not be clarified by the used experimental setting.

\subsection{Effects of kisspeptin-10 on proliferation}

The results in chapter 3.5 showed a reduced tumor growth by kisspeptin-10 treatment in vivo. The experiments of the following chapter describe the effect of KP-10 on proliferation in vitro. Therefore, breast cancer cell lines were investigated for their endogenous GPR54 expression. Their proliferation was studied under KP-10 treatment. For reasons of comparison, further experiments were carried out with cells transfected to overexpress GPR54. 


\subsubsection{GPR54 expression in breast cancer cells and transfected cells}

GPR54 receptor expression was studied by immune cytochemistry, mRNA and protein analysis (see chapter 2.2.4.1, 2.2.3.1-2.2.3.4 and 2.2.4.2).

\subsubsection{Receptor detection in breast cancer cells}

Figure 3.24 shows the results of the immune cytochemical staining. All cell lines expressed GPR54, visualized by the red staining with GPR54 antibody.
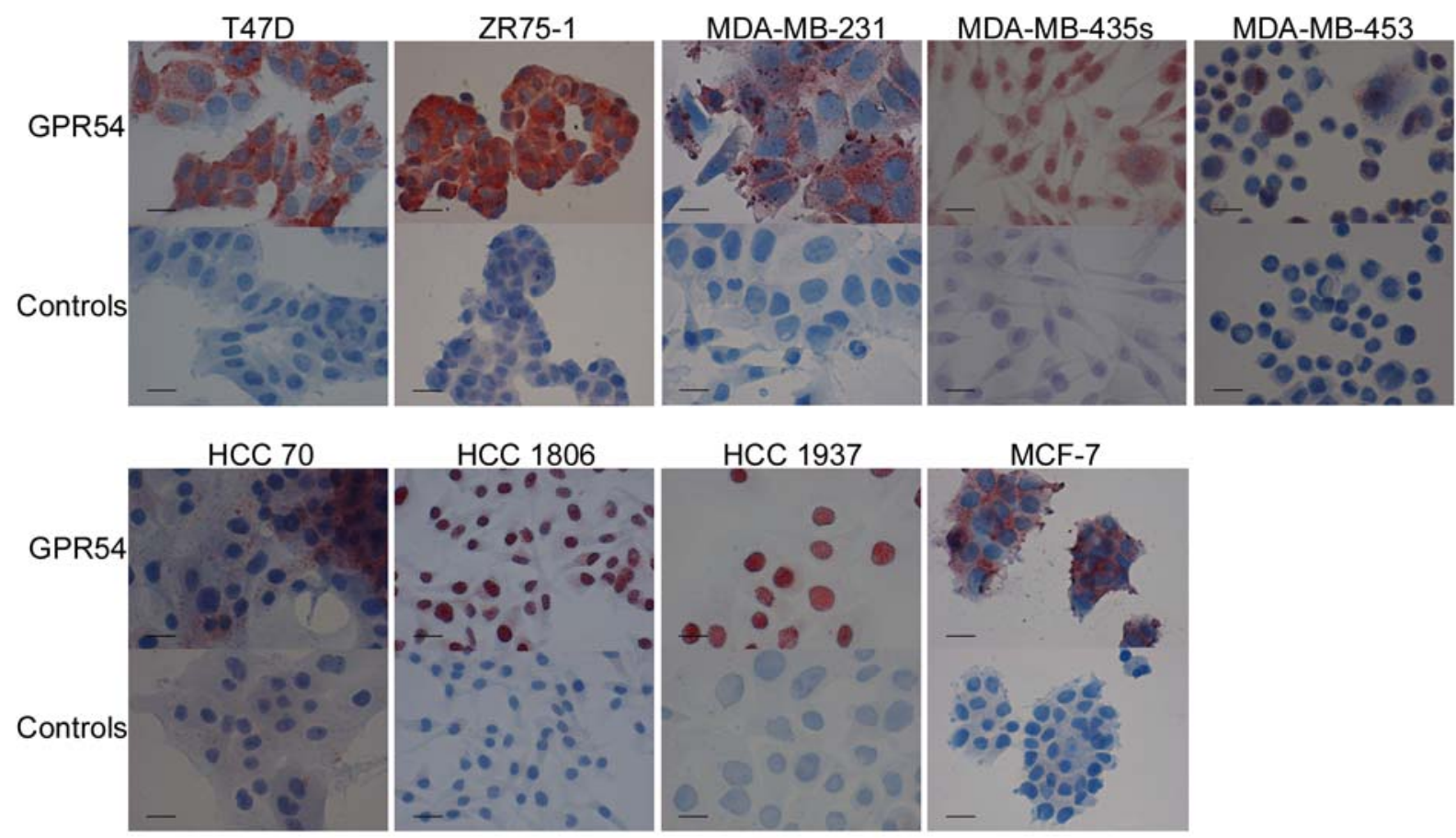

Figure 3.24: GPR54 expression in breast cancer cell lines

Breast cancer cell lines T47D, ZR75-1, MDA-MB-231, MDA-MB-435s, MDA-MB-453, HCC 70, HCC 1806, HCC 1937 and MCF-7 were grown on microscope chamber slides. GPR54 was detected immunocytochemically by red staining with the corresponding antibody (upper lines). Controls were performed by omission of the primary antibody (lower lines). Bright field images were taken (scale bar $=40 \mu \mathrm{m}$ ). Images represent the findings in at least three different passages of each cell line. This figure is published in Ziegler et al. 2013.

Further investigations on mRNA levels are represented in figure 3.25. T47D, ZR75-1 and MCF-7 showed receptor mRNA expression. In MDA-MB-231, MDA-MB-435s, MDA-MB-453, HCC 70, HCC 1806 and HCC 1937 no clear signal for GPR54 mRNA was found. In addition, figure 3.25 shows GPR54 expression of B35 clone 1 transfected to overexpress GPR54 (see chapter 3.6.1.2). The initial amount of cDNA of the clone used for mRNA analysis was 100 times smaller than the amount of cDNA used for the breast cancer cell lines. This was documented by the low signal 
of the housekeeping gene $\mathrm{L} 7$ in the clone samples compared to the breast cancer cell samples whereas the signal for GPR54 was clearly detectable.

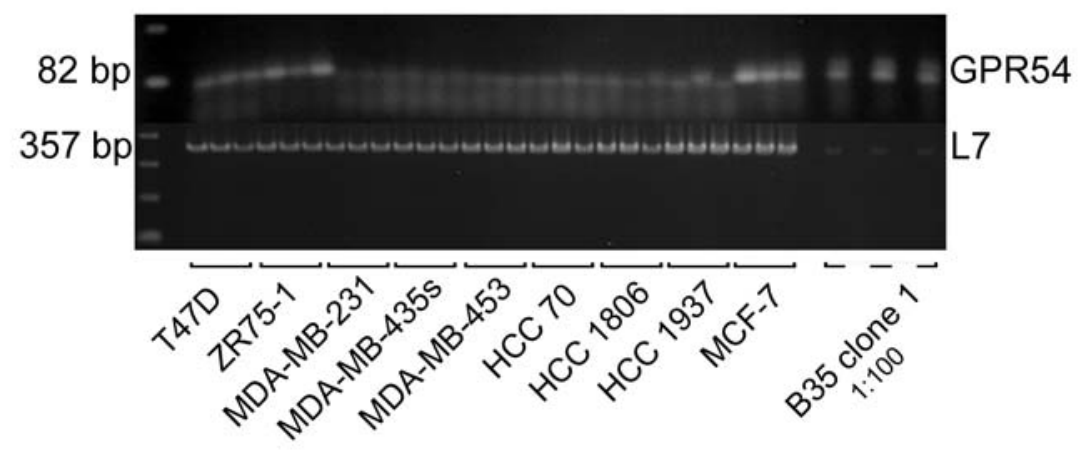

Figure 3.25: GPR54 mRNA expression

GPR54 mRNA expression was investigated by gene expression analysis via RT-PCR for breast cancer cell lines and B35 clone 1 transfected to overexpress GPR54 (cDNA dilution 1:100). For reasons of comparison, mRNA controls were performed by proof of the housekeeping gene L7. Images represent the findings in at least three different passages of each cell line. This figure is published in Ziegler et al. 2013.

Protein expression of GPR54 is imaged in figure 3.26. Protein levels were detected in every breast cancer cell line with different quantities. For reasons of comparison, GPR54 protein expression of B35 clone 1 was also detected.

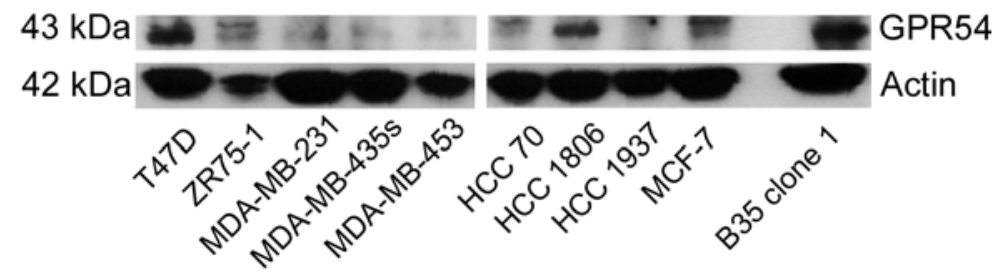

Figure 3.26: GPR54 protein expression

GPR54 protein expression was studied by Western blot analysis for breast cancer cell lines and B35 clone 1 (30 $\mu \mathrm{g}$ protein per sample). Controls were performed by proof of actin as protein standard. The image is representable for findings in at least three different passages of each cell line respectively clone. This figure is published in Ziegler et al. 2013.

\subsubsection{Receptor detection in cells transfected with GPR54}

B35 neuronal rat cells stable transfected with murine GPR54 were chosen as an artificial cell model overexpressing GPR54. Six clones of transfected B35 cells were tested for their GPR54 expression levels.

Results of the mRNA analysis are shown in figure 3.27. Clone $2(0.72 \pm 0.16$ SEM), clone 4 (1.36 \pm 0.61 SEM), clone $5(1.32 \pm 0.24$ SEM) and clone $6(0.75 \pm 0.16$ SEM) expressed GPR54 in 
a similar quantity compared to clone 1 . GPR54 expression level in clone 3 was significantly higher $(2.74 \pm 0.70 \mathrm{SEM})$ than in clone 1.

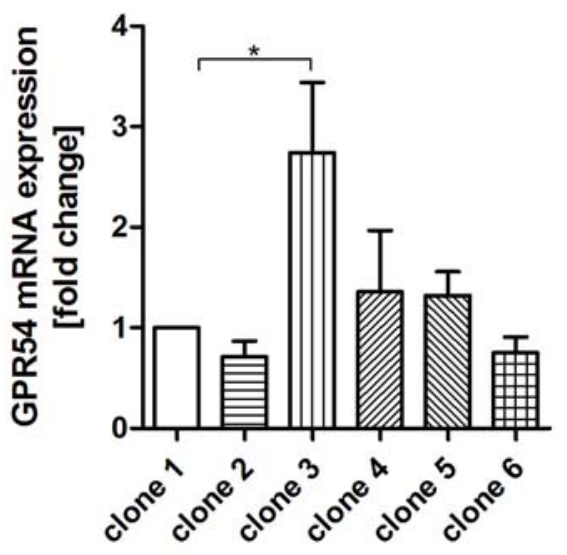

(a) Relative mRNA expression levels of GPR54

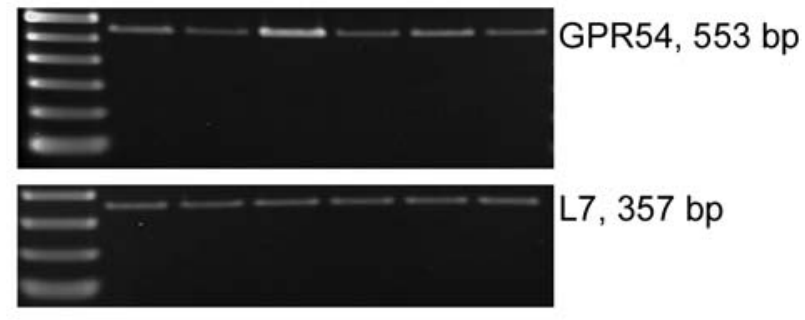

(b) Agarose gel of GPR54 expression

Figure 3.27: GPR54 mRNA expression in B35 mGPR54 clones

GPR54 distribution was investigated in six B35 mGPR54 clones by gene expression analysis via RT-PCR. (a) Expression was standardized to the expression of the housekeeping gene L7 and normalized to the expression of clone 1 (white column). Results were analyzed by 1way ANOVA and Dunnett's multiple comparison test (mean \pm SEM; *: $\mathrm{p}<0.05 ; \mathrm{n}=3$ ). (b) Images of agarose gels showing the PCR products of GPR54 and the housekeeping gene L7. Images represent the findings in at least three different passages of each clone. These figures are published in Ziegler et al. 2013.

On protein levels, no significant differences of GPR54 expression were observed in clone $2(0.70 \pm$ 0.20 SEM), clone $3(1.12 \pm 0.22$ SEM) clone 4 (1.47 \pm 0.40 SEM), clone $5(1.34 \pm 0.20$ SEM) and clone 6 (1.02 \pm 0.24 SEM; see figure 3.28) compared to clone 1. 


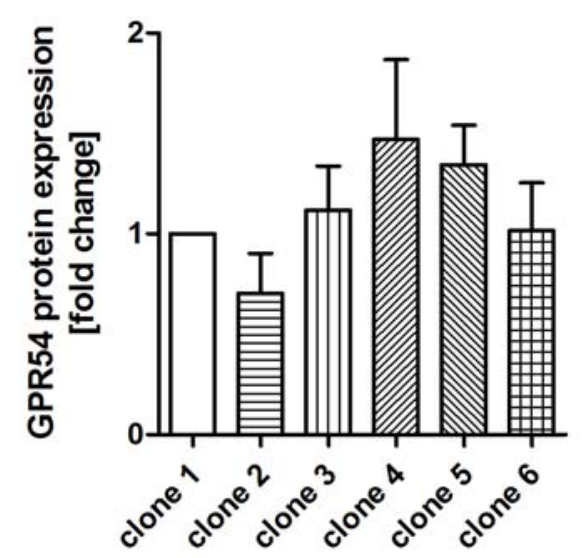

(a) Relative protein expression levels of GPR54

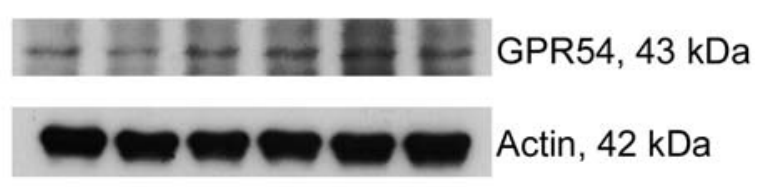

(b) Western blot of GPR54 expression

Figure 3.28: GPR54 protein expression in B35 mGPR54 clones

GPR54 protein expression was studied in six B35 mGPR54 clones by Western blot analysis. (a) Expression was standardized to the expression of actin and normalized to the expression of clone 1 (white column). Results were analyzed by 1way ANOVA and Dunnett's multiple comparison test (mean $\pm S E M ; n=3$ ). (b) Images of Western blots showing GPR54 and actin proteins. Images represent the findings in at least three different passages of each clone. These figures are published in Ziegler et al. 2013.

\subsubsection{Cell growth under kisspeptin-10 treatment}

For proliferation studies, four breast cancer cell lines and three B35 cell clones transfected to overexpress GPR54 were chosen. Proliferation was measured after treatment with KP-10 in different concentrations. KP-10 solutions were added once daily. Proliferation assays were prepared as described in chapter 2.2 .5 after $72 \mathrm{~h}$.

\subsubsection{Proliferation of breast cancer cells treated with kisspeptin-10}

MDA-MB-231, MDA-MB-435s, HCC 1806 and MCF-7 showed different GPR54 expression levels according to the results on mRNA and protein levels (see chapter 3.6.1.1). Proliferation was measured in controls and treated samples. No significant differences were observed for each cell line (MDA-MB-231: $100.0 \% \pm 0.7 \%$ SEM; $10^{-11} \mathrm{M}: 100.1 \% \pm 0.8 \%$ SEM; $10^{-9} \mathrm{M}: 101.1 \%$ $\pm 1.0 \%$ SEM; $10^{-7} \mathrm{M}: 100.3 \% \pm 1.9 \%$ SEM; $10^{-5} \mathrm{M}: 96.4 \% \pm 2.6 \%$ SEM; MDA-MB-435s: $100.0 \% \pm 2.7 \%$ SEM; $10^{-11} \mathrm{M}: 99.3 \% \pm 3.2 \%$ SEM; $10^{-9} \mathrm{M}: 100.1 \% \pm 4.1 \% \mathrm{SEM} ; 10^{-7} \mathrm{M}$ : $100.2 \% \pm 4.6 \%$ SEM; $10^{-5} \mathrm{M}: 101.5 \% \pm 7.0 \%$ SEM; HCC 1806: $100.0 \% \pm 1.2 \%$ SEM; $10^{-11} \mathrm{M}: 100.5 \% \pm 1.6 \% \mathrm{SEM} ; 10^{-9} \mathrm{M}: 96.6 \% \pm 2.6 \% \mathrm{SEM} ; 10^{-7} \mathrm{M}: 99.1 \% \pm 1.9 \% \mathrm{SEM}$; $10^{-5} \mathrm{M}: 99.0 \% \pm 3.9 \%$ SEM; MCF-7: $100.0 \% \pm 0.7 \%$ SEM; $10^{-11} \mathrm{M}: 99.7 \% \pm 2.4 \%$ SEM; $10^{-9} \mathrm{M}: 95.5 \% \pm 2.1 \%$ SEM; $10^{-7} \mathrm{M}: 99.5 \% \pm 4.9 \%$ SEM; $10^{-5} \mathrm{M}: 105.8 \% \pm 3.9 \%$ SEM; see figure 3.29 ). 


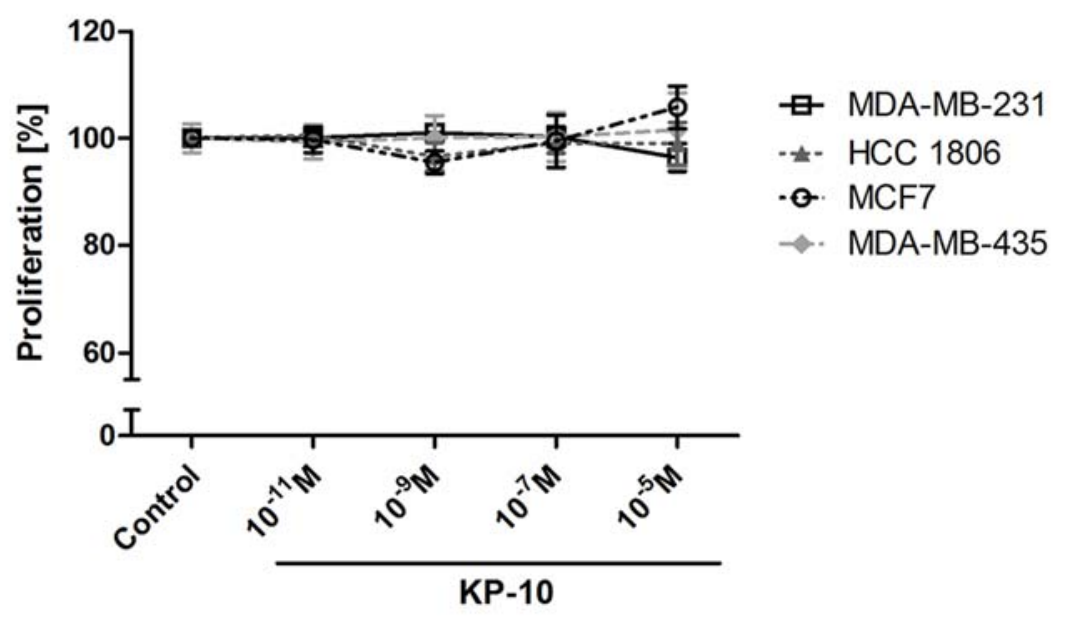

Figure 3.29: Proliferation of breast cancer cells treated with KP-10

The breast cancer cell lines MDA-MB-231, MDA-MB-435s, HCC 1806 and MCF-7 were grown in 96 well-plates and treated with KP-10 daily in different concentrations for $72 \mathrm{~h}$. Proliferation was normalized to the growth rate of the untreated controls. Results were analyzed by 1way ANOVA and Dunnett's multiple comparison test within each cell line (mean $\pm S E M ; n=6 / 8$ ). This figure is published in Ziegler et al. 2013.

\subsubsection{Proliferation of cells overexpressing GPR54 treated with kisspeptin-10}

Proliferation of cells transfected to overexpress GPR54 was measured in B35 clone 1, clone 3 and clone 4. Clone 3 showed the highest mRNA levels of GPR54 and the largest protein amount was detected in clone 4 (see chapter 3.6.1.2).

Proliferation is graphed in figure 3.30. Proliferation of clone 1 was marginal affected by treatment with high concentrations of KP-10 compared to controls (control: $100.0 \% \pm 1.2 \% \mathrm{SEM} ; 10^{-11} \mathrm{M}$ : $100.9 \% \pm 5.0 \%$ SEM; $10^{-9} \mathrm{M}: 101.7 \% \pm 4.0 \%$ SEM; $10^{-7} \mathrm{M}: 95.1 \% \pm 3.6 \% \mathrm{SEM} ; 10^{-5} \mathrm{M}$ : $91.3 \% \pm 1.8 \% \mathrm{SEM})$. The proliferation of clone 3 and clone 4 was significantly inhibited by KP-10 concentrations of $10^{-7} \mathrm{M}$ and $10^{-5} \mathrm{M}$ (clone 3: control: $100.0 \% \pm 1.8 \% \mathrm{SEM} ; 10^{-11} \mathrm{M}: 101.3 \%$ $\pm 1.4 \%$ SEM; $10^{-9} \mathrm{M}: 99.3 \% \pm 2.7 \%$ SEM; $10^{-7} \mathrm{M}: 83.5 \% \pm 1.6 \% \mathrm{SEM} ; 10^{-5} \mathrm{M}: 80.0 \% \pm$ $1.8 \%$ SEM; clone 4: control: $100.0 \% \pm 1.5 \%$ SEM; $10^{-11} \mathrm{M}: 98.2 \% \pm 2.8 \% \mathrm{SEM} ; 10^{-9} \mathrm{M}$ : $96.9 \% \pm 2.1 \%$ SEM; $10^{-7} \mathrm{M}: 87.5 \% \pm 4.1 \%$ SEM; $10^{-5} \mathrm{M}: 86.4 \% \pm 2.1 \%$ SEM). 


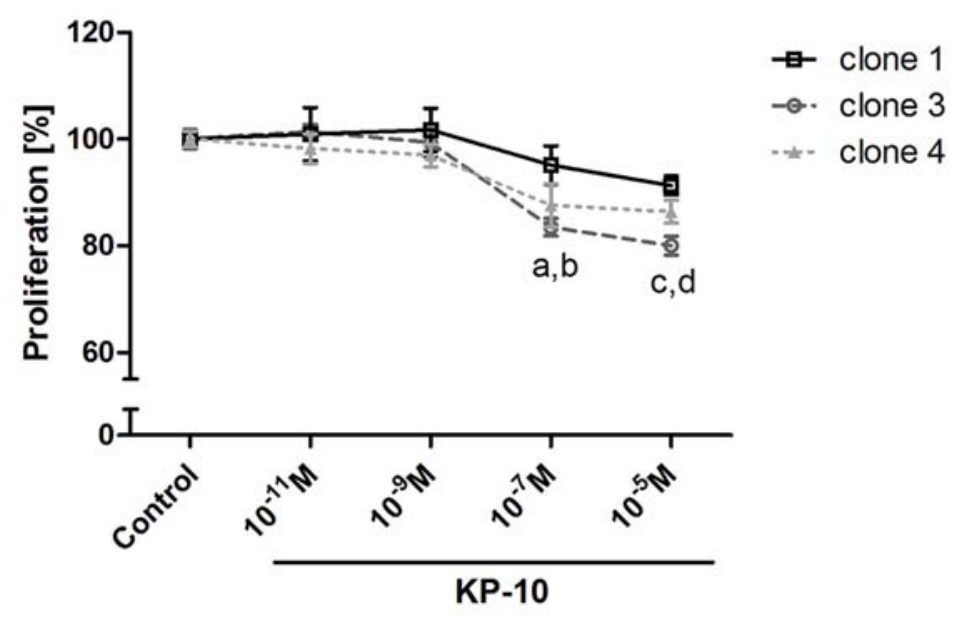

Figure 3.30: Proliferation of B35 mGPR54 clones treated with KP-10

The B35 clones 1, 3 and 4 were grown in 96 well-plates and treated with KP-10 daily in different concentrations for $72 \mathrm{~h}$. Proliferation was normalized to the growth rate of the untreated controls. Results were analyzed by 1 way ANOVA and Dunnett's multiple comparison test within each clone (mean \pm SEM; $a, c: p<0.001$ for clone 3 vs. control; $b: p<0.01$ for clone 4 vs. control; $d: p<0.001$ for clone 4 vs. control; $n=6 / 8$ ). This figure is published in Ziegler et al. 2013.

Taken together, GPR54 was expressed in all of the studied breast cancer cell lines. Results of different analytical methods vary in the amount of cellular receptor expression and a direct comparison of GPR54 levels was not possible. It seemed that GPR54 expression in the breast cancer cell lines was lower than in the transfected clones. Proliferation studies showed inhibited cell growth by KP-10 in B35 clone 3 and clone 4, but not in MDA-MD-231, MDA-MB-435s, HCC 1806 and MCF-7 cells. 


\section{Discussion}

\subsection{Coculture effects on breast cancer cells}

The interaction of breast cancer and osteoblast-like osteosarcoma cells led to increased invasion properties of non-invasive cells in an established coculture system. One important system, the CXCL12/CXCR4 system, was already identified for the interaction between CXCR4 expressing breast cancer cells and CXCL12 secreting osteosarcoma cells [von Alten et al., 2006]. Further work with CXCL12 antibodies reduced the invasion partially, but it was not able to abolish it [Olbrich, 2010a]. Thus, the altered invasive characteristics should be further investigated for a better understanding of other factors involved.

An increased cell motility is often accompanied by morphological alterations and a modified gene expression. One important process, the EMT, is often associated with cancer progression leading to loss of cell-cell adhesions and an enhanced invasiveness [Christiansen and Rajasekaran, 2006]. Thus, analysis of motility marker involved in EMT was carried out.

\subsubsection{Classification of breast cancer cell lines}

The primary attempt was to classify breast cancer cell lines in epithelial or mesenchymal phenotypes to estimate their invasive character. Cells can be differentiated by morphology, physical characteristics, intracellular junctions, molecular marker expression and cytoskeletal organization. Two aspects were chosen. Breast cancer cell lines were characterized for their morphological appearance and the expression of epithelial and mesenchymal marker. It was expected, that welldifferentiated cells growing in clusters with strong cell-cell contacts would express more epithelial marker. On the contrary, cells with a more spindle-like morphology and less differentiation would express mainly mesenchymal marker. The marker were chosen based on gene expression analysis in literature correlating CDH1 and TJP1 with an epithelial phenotype and VIM and S100A4 with a mesenchymal phenotype [Wu and Zhou, 2008, Kalluri and Weinberg, 2009]. CDH1 is a transmembrane protein involved in cell-cell adhesions by adherents junctions. Loss of that protein is 
highly associated with an invasive phenotype. TJP1 forms interconnections between single cells as permeability barriers for a restricted lateral diffusion. Its function is important for membrane polarity. The intermediate filament VIM facilitates cytoskeletal remodeling and is linked to an increased cell invasiveness. S100A4 belongs to the S100 family, which is responsible for motility, invasion and tubulin polymerization [Christiansen and Rajasekaran, 2006]. All of the studied marker vary in their cellular functions and were selected to check the expression from different starting points.

Seven of the nine tested breast cancer cell lines, T47D, ZR75-1, MDA-MB-453, HCC 70, HCC 1806, HCC 1937 and MCF-7, showed a more epithelial-like morphology, whereas MDA-MB-231 and MDA-MB-435s had a more mesenchymal-like appearance (see chapter 3.1.1). The presence of the two epithelial marker were analyzed in all of the cell lines. CDH1 expression correlated well with the morphological findings showing a strong expression in the first seven cell lines and very low respectively no amounts in MDA-MB-231 and MDA-MB-435s cells. The detection of TJP1 was not meaningful in this experimental setting. It was expressed in all of the cell lines without any observable difference. TJP1 was not a suitable marker in this context. Mesenchymal marker expression showed a correlation for VIM with the morphological appearance of the cells. Its strongest amount was detected in MDA-MB-231 and MDA-MB-435s cells. The other cell lines showed less or no expression. S100A4 showed different expression patterns in the cell lines. No direct link could be seen between its presence and the morphological phenotype of the cells (see chapter 3.1.2).

Based on these results, the breast cancer cell lines T47D, ZR75-1, MDA-MB-453, HCC 70, HCC 1806, HCC 1937 and MCF-7 were classified as epithelial-like cell lines. The results of MDA-MB-231 and MDA-MB-435s cells demonstrated these cell lines as mesenchymal-like. These findings are comparable with diverse studies in literature. Based on morphological analysis, T47D, ZR75-1 and MCF-7 cells showed a fused appearance in Matrige ${ }^{T M}$, whereas the cell lines MDA-MB-231 and MDA-MB-435s had a stellate morphology [Sommers et al., 1994, Zajchowski et al., 2001]. Gene expression profiling led to a clustering of breast cancer cells in luminal and basal cell lines. The luminal part was more differentiated and connected by tight cell-cell junctions identified for T47D, ZR75-1, MDA-MB-453 and MCF-7 cells. The basal phenotype was characterized by less differentiation and a mesenchymal-like appearance detected in MDA-MB-231 [CharafeJauffret et al., 2006, Neve et al., 2006]. In addition, gene analysis in T47D, ZR75-1 and MCF-7 cells showed expression of $C D H 1$, but no expression of VIM. The expression pattern of these two genes was found in the cell lines MDA-MB-231 and MDA-MB-435s vice versa [Sommers et al., 1994, Zajchowski et al., 2001]. This is consistent with the observations in the present work. In MDA-MB-453 cells, no CDH1 and no VIM was expressed [Sommers et al., 1994, Zajchowski et al., 2001], whereas the present results showed low expression rates of these genes in MDA-MB-453 cells. One study characterized the cell line HCC 1937 as basal respectively mesenchymal-like based 
on gene expression analysis. HCC 1937 cells expressed high amounts of VIM, MMP2, FN1 and S100A2, another member of the S100 protein family. All of the genes were related to the less differentiated phenotype [Charafe-Jauffret et al., 2006]. In the present work, HCC 1937 cells were classified as a more epithelial-like cell line adapted from the high expression rate of CDH1 and its morphology showing growth in clusters with direct cell-cell contacts. However, a clear expression of VIM was also observed and an absolute definition of this cell line can not be offered.

Although breast cancer cell lines were classified by several studies in literature, an analysis of the used cell lines was necessary due to possible changes caused by cultivation and growing. The media used in the different laboratories and the culture conditions varied. Furthermore, genetical changes over years of cultivation were possible and could have led to different behavior and phenotypes. Therefore, the cell lines were used for experiments in early passages (except for MDA-MB-453) to maintain their characteristics. Comparison with the results available in literature showed a good analogy for most of the cell lines used in the present work.

\subsubsection{Invasive and migratory behavior}

In addition to the morphological and gene expression analysis of the nine breast cancer cell lines, they were studied for their invasive properties. The morphological phenotype and the invasive behavior of cells were shown to be interdependent. A more mesenchymal-like respectively basal appearance was associated with high invasiveness and a more epithelial-like respectively luminal morphology was connected with low or no invasion [Sommers et al., 1994, Zajchowski et al., 2001, Neve et al., 2006, Cheng et al., 2007]. For the epithelial-like classified cell lines, T47D, ZR75-1, MDA-MB-453, HCC 70, HCC 1806, HCC 1937 and MCF-7, less invasion was estimated. Whereas the more mesenchymal-like cell lines MDA-MD-231 and MDA-MB-435s were expected to show strong invasion.

During the first experiments, the individual invasion was investigated of each cell line (see chapter 3.2.1). The strongest cell movement was identified for the cell lines MDA-MB-435s and MDA-MB-231. An intermediate invasion was detected in HCC 70, HCC 1806 and HCC 1937 and almost no invasion was observed in T47D, ZR75-1, MDA-MB-453 and MCF-7 cells. These findings correlated well with the observed phenotypical characteristics and EMT marker expression in the present study. Studies in literature showed consistent results classifying the cell lines T47D, ZR75-1, MDA-MB-453 and MCF-7 as less or poorly invasive and MDA-MB-231 and MDA-MB-435s as moderately respectively highly invasive [Neve et al., 2006, Kokkinos et al., 2007].

Further experiments of the present work focused on the invasion in coculture. Therefore, breast cancer cells were grown with an osteoblast-like osteosarcoma cell line without direct cell-cell contact. Invasion increased in all of the cell lines except for MDA-MB-231 and MDA-MB435s. In 
comparison with their individual invasion, T47D and MCF-7 showed the largest increase in their invasive properties with an raise of more than 300 times. Based on these observations, invasion of four different behaving cell lines, MCF-7, HCC 1806, MDA-MB-231 and MDA-MB435s, was studied in detail (see chapter 3.2.2). The non-invasive cell line MCF-7 and the intermediate invasive cell line HCC 1806 showed an increased invasion already after $48 \mathrm{~h}$ under coculture conditions. MDA-MB-231 and MDA-MB435s were decreased in their invasion by cocultivation after $96 \mathrm{~h}$. But the detailed analysis showed an increase in the invasive behavior for the first $48 \mathrm{~h}$ of coculture. After $72 \mathrm{~h}$, no differences in invasion could be observed between both conditions and after $96 \mathrm{~h}$, the invasion in monoculture was higher. In literature, related experimental settings were described focusing an interaction of breast cancer cell lines with other cells respectively tissues. Cancer-associated fibroblasts (CAFs) are cells arising from tumor stroma. They can interact with the tumor cells by release of soluble factors as TGF $\beta$, hepatocyte growth factor (HGF) and insulin-like growth factors (IGFs). Thereby, CAFs were shown to promote cancer cell proliferation and invasion. Coculture studies with CAFs led to increased migration of PMC42-LA, MCF-7 and MDA-MB-231 breast cancer cells [Lebret et al., 2007, Angelucci et al., 2012]. Other experiments with conditioned media from NIH3T3 murine fibroblasts showed only low invasion and migration of T47D, ZR75-1, MDA-MB-453 and MCF-7 cells, whereas the invasion of MDA-MB-231 and MDA-MB435s was high respectively intermediate [Sommers et al., 1994, Zajchowski et al., 2001]. The influence on invasion seems to be dependent on the kind of interacting cells respectively tissues showing different changes. An explanation for the "switch" of the invasive behavior of MDA-MB-231 and MDA-MB-435s cells during cocultivation may be, that one of the factors released by the osteosarcoma cells leads to a receptor internalization caused by an enhanced stimulation over time. Further analysis of factors triggering invasion in coculture is discussed in chapter 4.1.3.

Migration analysis of MCF-7 and MDA-MB-231 cells cocultivated with CAFs showed a more directed cell movement [Angelucci et al., 2012]. According to this, experiments with MCF-7 cells showed no influence on migratory behavior by cocultivation with osteosarcoma cells in the present work (see chapter 3.2.2.3). Thus, the interaction of breast cancer cells with osteosarcoma cells seems to be important in three-dimensional cell movement leading to a "bone"directed invasion in the studied coculture system. Studies on bone-directed invasion identified the CXCL12/CXCR4 system as a potent regulating system. Earlier works revealed an involvement of the CXCL12/CXCR4 system in the coculture system [von Alten et al., 2006]. In addition, CXCL12 was identified in bone marrow and its protein extract respectively CXCL12 treatment led to increased invasion and migration of CXCR4-expressing MDA-MB-231 cells [Müller et al., 2001]. CXCL12 treatment was also shown to change morphology of oral squamous carcinoma cells to a mesenchymal-like phenotype and to increase chemotaxis [Uchida et al., 2003, Onoue et al., 2006]. An increase in chemotactic migration by CXCL12 was observed in CXCR4-expressing 
ovarian carcinoma cells as well [Scotton et al., 2001]. Further experiments of the present work on gene expression of cocultured breast cancer cells are discussed in chapter 4.1.3.

Another experimental setting of the present work was carried out according to evidence in literature on EMT triggered by TGF $\beta$. TGF $\beta$ is known as tumor suppressor in early tumor development, but later on, it is promoting cancer progression. Invasion is increased by induction of EMT via TGF $\beta$ [Zavadil and Bottinger, 2005, Iwatsuki et al., 2010, Taylor et al., 2010, Drabsch and Dijke, 2011]. Rat hepatoma cells were treated permanently with TGF $\beta$ leading to a morphological conversion from epithelial-like cells to a more mesenchymal-like phenotype. Their invasion and migration was increased against conditioned media from fibroblasts and osteoclasts [Bertran et al., 2009]. Similar effects were observed in squamous carcinoma cells treated with TGF $\beta$. Their cellular morphology was changed and their migratory properties were increased [Taki et al., 2008]. In the present work, breast cancer cells were treated with $\mathrm{TGF} \beta_{1}$ under monoculture and coculture conditions to test whether the invasion rate could be increased. This was important for further experiments on gene expression analysis upon an involvement of EMT in invasion processes during cocultivation (see chapter 4.1.3). TGF $\beta_{1}$ affected invasion was studied in the cell lines MDA-MB-231, HCC 1806 and MCF-7. In monoculture, an increased invasion was shown for HCC 1806 cells, but no changes were observed for MDA-MB-231 and MCF-7. No significant effects on invasion in coculture were identified for all of the three cell lines. Thus, $\operatorname{TGF} \beta_{1}$ regulation was not able to enhance invasion in the used experimental setting.

\subsubsection{Gene expression}

Gene expression analysis was carried out for a better understanding of the mechanisms leading to increased invasion of breast cancer cells cocultivated with osteoblast-like osteosarcoma cells. The focus was put on EMT as an important process of cell motility. In literature, several studies indicated activators of EMT. TGF $\beta$ was shown to induce morphological changes from an epitheliallike to a mesenchymal-like phenotype. It increased invasive and migratory properties of cells and changed the expression of genes involved in EMT as downregulation of $\mathrm{CDH} 1$ and upregulation of VIM [Sommers et al., 1994, Bertran et al., 2009]. The CXCL12/CXCR4 system seems to be also involved in EMT. Treatment with CXCL12 resulted in conversion of cell morphology, increased invasion and $\mathrm{CDH} 1$ downregulation respectively VIM upregulation [Uchida et al., 2003, Onoue et al., 2006]. In addition, coculture interactions of CAFs with cancer cells caused increased migration, downregulated $\mathrm{CDH} 1$ and upregulated VIM [Angelucci et al., 2012]. In the present work, another interaction should be studied in relation to EMT: the cocultivation of breast cancer and osteosarcoma cells leading to increased invasive properties.

Epithelial marker CDH1 and TJP1 and mesenchymal marker VIM and S100A4 were analyzed in MDA-MB-231, HCC 1806 and MCF-7 cells in monoculture and coculture. An additional treat- 
ment of cocultivated cells with $\mathrm{TGF} \beta_{1}$ was performed to induce changes of marker expression according to altered expression patterns generated by EMT (see chapter 3.2.3). These experiments should serve as positive controls for EMT. It was expected, that increased invasion was correlated with downregulation of epithelial marker and upregulation of mesenchymal marker. In the present work, CDH1, TJP1, VIM and S100A4 expression were not affected by cocultivation in MDA-MB-231 cells. Treatment with TGF $\beta_{1}$ did not influence expression of CDH1 and S100A4, but it upregulated TJP1 and VIM expression in this cell line. In HCC 1806 cells, coculture conditions led to no differences in CDH1, TJP1 and VIM expression, but expression of S100A4 was decreased. Exposure to TGF $\beta_{1}$ did not changed $\mathrm{CDH} 1$ expression, but upregulated TJP1 and VIM expression and downregulated expression of S100A4. Cocultivation of MCF-7 cells showed no effect on CDH1, TJP1 and VIM expression levels. It increased expression of S100A4. TGF $\beta_{1}$ treatment decreased CDH1, TJP1, VIM and S100A4 expression. These results indicated no influence on gene expression of CDH1, TJP1 and VIM by cocultivation in all of the cell lines. Only S100A4 was changed differently in HCC 1806 and MCF-7. These findings lead to the assumption, that the increased invasion of breast cancer cells seems not to be induced by mechanisms involved in EMT in the used coculture system. But the gene expression analyzed under TGF $\beta_{1}$ treatment also showed no homogeneous expression pattern for epithelial marker respectively mesenchymal marker, neither for all of the cell lines nor within one cell line. Expression was changed, but in every possible direction. One explanation may be, that $\mathrm{TGF} \beta_{1}$ is not a reliable inducer of EMT. In literature, there are studies on motility marker expression showing no correlation of expression changes by TGF $\beta$ for several cell lines. Invasion was not investigated [Brown et al., 2004, Chai et al., 2010]. But a lot of studies in literature support the opinion, that $\operatorname{TGF} \beta$ increases invasion by induction of EMT [Zavadil and Bottinger, 2005, Bertran et al., 2009, Iwatsuki et al., 2010, Taylor et al., 2010, Drabsch and Dijke, 2011]. In agreement with the high evidence of TGF $\beta$ induced EMT, another factor may be responsible for the inconsistent results in the experimental setting of the present work. On the one hand, the choice of motility marker may be not suitable for the analysis. As discussed in chapter 4.1.1, TJP1 and S100A4 did not correlate with the morphological phenotype of the cells, but $\mathrm{CDH} 1$ and VIM were acceptable. CDH1 and VIM were also studied well in literature showing good associations with the epithelial respectively mesenchymal phenotype and invasive properties [Sommers et al., 1994, Zajchowski et al., 2001]. Thus, their detection is maintainable in this context. On the other hand, the sample preparation may be insufficient. The coculture system consisted of two chambers. The bottom well was used for osteosarcoma cell cultivation. As upper well, a cell culture insert was used with a Matrigel ${ }^{T M}$ coated filter membrane. This membrane allowed medium exchange and an invasion of the breast cancer cells towards the bottom well. For RNA isolation, all of the breast cancer cells of the upper well were collected. This included cells, which invaded through the gel and other subpopulations ontop of the gel, which were not influenced by these conditions. But cells were not included in the sample, which invaded through the filter. It is possible, that the results of the gene expression 
analysis were overlaid by RNA of cell subpopulations which were not influenced by coculture conditions. This would explain, why the findings of the present work did not show an EMT induction by TGF $\beta_{1}$. According to this, it may be possible, that invasion in the coculture system influences expression of the motility marker as well.

In further experiments, the setting and RNA collection was optimized. Therefore, only cells at the bottom of the filter were collected, which were invaded through Matrige ${ }^{T M}$ and membrane. The cell line MCF-7 was used and the samples of monocultured and cocultured cells were investigated by microarray analysis (see chapter 3.3). 50 genes, which were regulated the most ( $88 \%$ were upregulated and $12 \%$ were downregulated), were classified into groups for their cellular functions. The main part of the genes belonged to immune response mechanisms as inflammation, viral infection and interferon dependent processes, and cell motility processes, adhesion and metastasis. The microarray data was validated for VIM, FN1, SPARC, MMP2, TCF21, CXCL12, PARP9 and TACC1 by qPCR. VIM and FN1 represented marker of EMT. FN1 belongs to the ECM proteins and its expression correlated with the mesenchymal-like phenotype [Wu and Zhou, 2008, Kalluri and Weinberg, 2009, Yilmaz and Christofori, 2010]. Another ECM protein is SPARC, also known as osteonectin (ON). It promoted EMT-like changes as decreased CDH1 and enhanced fibronectin and MMP expression together with an increased invasiveness and metastasis [Chen et al., 2012, Fenouille et al., 2012]. MMP2 degrades the ECM and is often secreted by invasive and mesenchymal-like cells [Kalluri and Weinberg, 2009, Yilmaz and Christofori, 2010, van Zijl et al., 2011]. TCF21 is a marker for mesenchymal cells. During tissue development, its expression was shown to be located at mesenchymal sites of EMT, but not in epithelial compartments [Quaggin et al., 1998]. The results of the microarray showed many regulated genes which were involved in immune response. According to this, PARP9 was selected. It is induced by inflammatory mediators like interferons. Expression of PARP9 led to upregulation of other interferon induced proteins as interferon induced protein with tetratricopeptide repeats (IFITs), 2'-5'-oligoadenylate synthetase (OASs) and signal transducer and activator of transcription 1 (STAT1) [Juszczynski et al., 2006]. PARP9 levels were enlarged in high-risk lymphomas and its overexpression enhanced migration of lymphoma cells independent of the CXCL12/CXCR4 system [Aguiar et al., 2000]. CXCL12 was chosen based on the high evidence in literature on an interaction of the CXCL12/CXCR4 system with invasion especially to bone tissue as protein extracts, cells or conditioned medium [Müller et al., 2001, Wang et al., 2006, Olbrich, 2010a]. TACC1, one of the genes which was downregulated by coculture conditions, represents a centrosomal protein. It interacts with microtubule organization [Gergely et al., 2000] and was less expressed in tumor tissue compared to normal tissue in breast, lung, ovarian and uterine cancer [Conte et al., 2002].

The present microarray data correlated well with the findings in literature for VIM, FN1, SPARC and MMP2. Thus, the cocultured cells showed a change to a more mesenchymal-like behavior. This may be one explanation for the increased invasive capabilities of the MCF-7 cells in the 
coculture system. In case of EMT, the results of the present work offer no link to a downregulation of epithelial marker as CDH1. In contrast to the supported opinion in literature that CDH1 downregulation is a "hallmark" in EMT [Kalluri and Weinberg, 2009, Yilmaz and Christofori, 2010, van Zijl et al., 2011], different studies indicated that loss of $\mathrm{CDH} 1$ was not necessarily required for invasion and migratory processes [Christiansen and Rajasekaran, 2006, Chen et al., 2007, Chai et al., 2010]. A conditioning of MCF-7 cells by repeated invasion cycles showed an increase in invasive properties compared to the parental cells. This was accompanied by an unchanged expression of $\mathrm{CDH} 1$. Comparison of gene expression profiles of the selected invasive MCF-7 cells with MDA-MB-231 cells revealed genes associated with a more invasive phenotype as CXCL12 and CD44 [Uchino et al., 2010]. These genes were also upregulated by coculture conditions in the present work. Evidence for a phenotypical conversion of the MCF-7 cells by cocultivation is also given by other factors regulated in the microarray data. TCF21 was upregulated, which is mainly expressed in mesenchymal cells. TACC1 was downregulated offering hints for a different microtubule organization. Therefore, a change to a more mesenchymal character of the MCF-7 cells seems to be performed by cocultivation with osteosarcoma cells without an alteration of epithelial properties. Thus, hints for an EMT were partially observed. In literature, an "incomplete" EMT is discussed. Evidence for a well-differentiated epithelial morphology in invasive and metastatic cancers is given. Thus, loss of epithelial characteristics seems not to be necessary for invasiveness [Christiansen and Rajasekaran, 2006]. An incomplete EMT had possibly occurred in the present work.

Another system involved in invasion is the CXCL12/CXCR4 system. In literature, there is great evidence showing high expression of CXCR4 in tumor cells which were attracted by secreted CXCL12 of the microenvironmental tissues and cells [Mantovani et al., 2010]. At the site of primary tumor in breast cancer, studies showed that CXCR4 was expressed in great amounts [Wang et al., 2006]. Whereas CXCL12 expression was at high levels in tissue as lymph nodes, lung, liver and bone marrow representing the most common sites of breast cancer metastasis. Migration and invasion of CXCR4 expressing MDA-MB-231 cells was increased against CXCL12 respectively protein extracts of lymph nodes, lung, liver and bone marrow. In vivo, treatment with CXCR4 antibodies decreased metastasis rates of MDA-MB-231 in mice [Müller et al., 2001]. The present data showed an increase in CXCL12 expression in the cocultured MCF-7 cells, but CXCR4 expression was not changed. Thus, there is no link to the common assumption. Further studies in literature revealed an interaction of endogenous CXCL12 with invasiveness. In breast cancer, CXCL12 levels correlated with prognosis, disease-free survival and overall survival of patients [Kang et al., 2005b, Mirisola et al., 2009]. Poor prognosis was also connected with CXCL12 positive cases in oral squamous cell carcinoma [Uchida et al., 2007]. Transfection of cells with CXCL12, which showed endogenous CXCR4 expression, led to increased invasion and migration in breast cancer, oral squamous carcinoma and non-small cell lung carcinoma cells [Kang et al., 2005b, 
Uchida et al., 2007, Dai et al., 2013]. Vice versa, knockdown of CXCL12 was accompanied by a reduced invasive and migratory behavior [Kang et al., 2005a]. CXCL12 expression was also shown to upregulate MMP expression [Singh et al., 2004, Shen et al., 2009, Dai et al., 2013]. In addition, an upregulation of MMP2 was observed in the cocultured MCF-7 cells in the present work. This represents an important mechanism of enhanced invasive properties by degradation of the ECM. The autocrine interaction of CXCL12 with its receptor leading to increased invasiveness was also detected in vivo and discussed as necessary for distant metastasis [Uchida et al., 2007]. The MCF-7 cells used in the present work expressed CXCR4 endogenously, which was shown in earlier works [von Alten et al., 2006, Olbrich et al., 2010b]. This leads to the assumption that an autocrine interaction of CXCL12 with CXCR4 is triggered by cocultivation of breast cancer cells with osteosarcoma cells. Thereby, the osteosarcoma cells affect the breast cancer cells to increase their CXCL12 expression. This seems to be one important aspect in the enhancement of invasiveness. The detailed mechanism and the involved factors are still not known.

A great part of the genes regulated by coculture conditions is involved in immune response processes as inflammation and infection. Evidence for a connection between these mechanisms and cancer is offered in literature. Chronic exposure to pathogens can lead to repeated injuries which facilitate acute and chronic inflammation. Hepatitis virus $B$ and $C$ infections were identified as risk factors for the development of hepatocellular carcinomas. Oncogenic papilloma virus was related to invasive cervical cancer. Factors interacting with interleukin receptors, toll-like receptors and the tumor necrosis factor (TNF) receptor family were associated with these processes [Karin et al., 2006]. Recruitment of bone marrow derived cells as tumor associated macrophages (TAMs) was also shown to enhance invasiveness and migration of tumor cells by secretion of epidermal growth factor (EGF). In a paracrine loop, the tumor cells responded by expressing colony stimulating factor 1 (CSF1), which attracted TAMs [Joyce and Pollard, 2009]. The microarray data of the present work revealed a slight upregulation of CSF1 in the cocultured breast cancer cells. According to this, EGF may represent one factor which triggers the enhanced invasiveness. Another gene involved in inflammatory processes, PARP9, was upregulated by cocultivation. It is one of the 50 genes showing the strongest regulations. Its expression led to upregulation of other interferon induced proteins as IFITs, OASs and STAT1 [Juszczynski et al., 2006]. These genes were also upregulated in the present microarray data. Interferon induced proteins were shown to increase migration in vitro together with enhanced MMPs activity by transfection of IFITM1 in colorectal cancer cells. In vivo, tumor volumes and weights were also enlarged [He et al., 2012]. In clinical samples of patients with metastatic carcinoma, the interferon regulated OAS were upregulated compared to healthy persons [Merritt et al., 1985]. STAT1 expression was induced by interferon leading to increased migration and invasion in breast cancer cells [Greenwood et al., 2012]. Interferon seems to be interacting in processes important for cellular invasion, migration and metastasis. Thus, it may be an interesting factor of investigation in the coculture studies of 
the present work.

Further research in literature on culture effects on invasive properties showed similar effects as observed in the present work. In breast cancer cells, an increased migration concomitant with an upregulated VIM expression was induced by cocultivation with CAFs [Lebret et al., 2007]. Cocultivation of MCF-7 cells with macrophages led to increased invasion under treatment with endothelin 1 (ET1) and upregulated expression of MMP2 and MMP9. Expression of ETs was higher in tumor tissue at the invasive front [Grimshaw et al., 2004]. Enlarged ET1 and MMP2 expression were also detected in the present work. Gene expression analysis of cocultured mammary adenocarcinoma cells with pre-osteoblastic cells resulted in upregulation of genes involved in wound healing processes, cell migration and adhesion processes [Glait-Santar et al., 2012]. The genes VIM, serpin peptidase inhibitor, clade E, member 2 (SerpinE2) and CD44 were also affected in the coculture system of the present work. SerpinE2 was shown to be involved in MMP expression and metastasis in breast cancer [Fayard et al., 2009]. CD44 expression as cell-surface glycoprotein was correlated with high invasiveness and accompanied by enhanced expression rates of CXCR4, MMP1 and connective tissue growth factor (CTGF) in breast cancer cells [Sheridan et al., 2006]. CD44 transfected cells showed increased migration and invasion in vitro and higher mortality rates and metastasis in vivo. Upregulation of CD44 is associated with increased production of FN1 and collagens [Rutnam and Yang, 2012]. Additionally, the present microarray demonstrated an upregulation of CD44 and CTGF by cocultivation. CTGF, MMP1, CXCR4 and others were also shown to be responsible for increased bone metastasis of breast cancer cells [Kang et al., 2003]. Thus, cellular mechanisms including CD44 and CTGF seem to play a role in the coculture system used in the present work.

\subsection{Kisspeptin-10 and breast cancer}

Kisspeptin-10, a peptide derived from the metastasis suppressor gene KISS1, was shown to inhibit invasion in the coculture system of breast cancer cells and osteoblast-like osteosarcoma cells [Olbrich et al., 2010b, Olbrich, 2010a]. Further studies on KP-10 in vivo were carried out to investigate an antimetastatic effect on breast cancer xenografts by peripheral administration.

\subsubsection{In vitro effects on invasion}

Several studies in literature reported decreasing effects of KP-10 on invasion in different cell lines and different invasion respectively migration assays. In trophoblasts, inhibited invasion was observed in a transwell invasion assay [Bilban et al., 2004]. Invasion of renal cell carcinoma cells was reduced by KP-10 in an invasion assay against FCS [Chen et al., 2011]. In Chinese 
hamster ovary $\mathrm{CHO}$ cells transfected with hGPR54, KP-10 inhibited migration via interaction with its receptor [Hori et al., 2001]. In addition, in cells expressing GPR54 endogenously invasion respectively migration was decreased by KP-10 [Shoji et al., 2009, Kang et al., 2011, Roseweir et al., 2012]. An in vitro effect of KP-10 on invasion of the breast cancer cell lines MCF-7 and MDA-MB-231 was shown in earlier works [Olbrich et al., 2010b, Olbrich, 2010a]. Another breast cancer cell line, HCC 1806, should be also used in vivo. Therefore, an effect of KP-10 in cocultivated HCC 1806 cells was investigated in preliminary works. The results did not show a significant inhibition of invasion in this breast cancer cell line by KP-10 (see chapter 3.4.1).

In parallel works, an analog of KP-10 was studied in the coculture system. The Phe at position six of the peptide was substituted by dLys in order to create an effective analog with a possible longer half life. The half life of KP-10 was shown to be very short especially in serum. This is mainly caused by degradation by MMPs [Tomita et al., 2008]. Studies in literature demonstrated a more potent KP-10 analog by the exchange of enantiomers from L-amino to D-amino. The replacement of Tyr against $\mathrm{dTy}$ at position one resulted in an extended effect compared to the original peptide in vivo [Curtis et al., 2009]. During the development of analogs, it was important to keep the C-terminal end of the peptide, because especially Arg and Phe at positions nine respectively ten were shown to be critical for receptor activity [Clements et al., 2001, Kotani et al., 2001, Orsini et al., 2007]. The KP-10 analog DKP-10 inhibited invasion of HCC 1806 cells significantly in the coculture system (see chapter 3.4.2). The effect was related to the observed concentration window in the cell line MCF-7 [Olbrich et al., 2010b]. Thus, effectiveness of DKP-10 was proved in vitro. The effect was even stronger compared to KP-10 in HCC 1806 cells. The original peptide did not show a significant inhibition of invasion, only a trend was observed in that cell line.

\subsubsection{In vivo effects on tumor growth and metastasis}

In literature, an antimetastatic effect of KP-10 respectively KISS1 in vivo was shown by transfection of the gene in melanoma [Lee et al., 1996, Lee and Welch, 1997a] and breast [Lee and Welch, 1997b], ovarian [Jiang et al., 2005] and pancreatic cancer cells [McNally et al., 2010]. Reduced metastasis by peripherally administered KP-10 was studied in xenografts with melanoma [Ohtaki et al., 2001], prostate [Cho et al., 2009b] and endometrial cancer cells [Kang et al., 2011]. In the present work, the effect of a peripheral treatment with KP-10 should be investigated in breast cancer xenografts. Evidence for a possible role of KP-10 as therapeutic option in the treatment of metastatic breast cancer should be studied. As breast cancer is not a homogeneous disease, the experimental setting covered different breast cancer xenografts with varying cell lines and two kinds of tumor cell injections. Metastasis should be studied in bone, lung and liver representing the most common sites of tumor cell settlement in breast cancer patients [Disibio and French, 2008]. The metastatic lesions in murine organs were analyzed by quantitative real-time PCR after DNA 
isolation. Target sequence was the ALU element, which is specific for primate genomes [Batzer and Deininger, 2002]. Thus, only human DNA of the breast cancer cells, which were injected in mice, was detected by this method. Metastasis was determined as ratio of human DNA in murine DNA. In contrast to histological analysis of metastasis, this sensitive assay enables detection of micrometastases by differentiation of species specific DNA and is not limited to a minimum extent of the metastases [McKenzie et al., 1991, Shoemaker et al., 1992, Schneider et al., 2002].

One of the xenograft models was performed by intracardiac injection. This assay was chosen as metastasis model for the later steps during the metastasis cascade. The tumor cells were injected directly into the bloodstream to study organ specific metastasis without primary tumor development. The breast cancer cell line MDA-MB-231 was used, which was shown to metastasize preferentially to bone and liver in nude mice by intracardiac injection [Sasaki et al., 1995, Yoneda, 2000, Richert et al., 2009]. This cell line was also affected by KP-10 in vitro [Olbrich, 2010a]. In the present work, no metastasis were detected in lung, liver and bone and no CTCs were found. As animal models and cellular systems are very sensible experimental settings, it is possible, that this kind of assay do not work anytime and anywhere. The mice would have reacted unlikely with the tumor cell injections. The cells of the used passage would have been unable to settle down at distant organ sites. And, maybe, the cell preparations respectively the injection technique were not sufficient. The analytical assay worked based on detection of positive control samples. According to this, the intracardiac injection of MDA-MB-231 cells in mice did not worked for studying metastasis and the effect of KP-10 in the present work.

Another xenograft model was carried out by orthotopic tumor cell injection into the mammary glands of the mice. According to this, the whole metastasis cascade could be passed through from primary tumor growth until metastatic colonization at distant sites. Two breast cancer cell lines were used, MDA-MB-435s and HCC 1806. MDA-MB-435s cells were shown to develop tumors and metastasis to bone, lung and liver after orthotopic injection [Price and Zhang, 1990, Castillo-Pichardo et al., 2009, Schubert et al., 2011]. This cell line was discussed controversially in literature for being not a breast cancer but a melanoma cell line by contamination or mistake. Gene expression analysis revealed an expression pattern more likely for a melanoma origin [Ellison et al., 2002, Rae et al., 2004]. But others reported, that the melanoma cell line, which should have been responsible for the contamination, was of male origin. Analysis of the MDA-MB-435 cell line revealed a female origin. Thus, the melanoma cell line seemed to be contaminated by the breast cancer cell line [Chambers, 2009]. According to this, MDA-MB-435s cells were used as breast cancer cells within this work.

The cell line HCC 1806 was also proved for tumor growth and metastasis to lung and liver [Föst et al., 2011, Volk-Draper et al., 2012]. An influence of KP-10 on HCC 1806 cells was investigated showing a trend towards lower invasion (see chapter 3.4.1). Because of the different invasive behavior of cocultured MDA-MB-435s (see chapter 3.2.1), these cells were not studied for an 
effect of KP-10 in vitro. The results of the present work demonstrated a significantly inhibited tumor growth of HCC 1806 cells in mice by KP-10 treatment. Metastases were detected in lung and CTCs were found in blood. No difference in treated and untreated mice were determined for both sites. In bone and liver, no hDNA was found (see chapter 3.5.2). In xenografts with MDA-MB-435s cells, tumor growth in the treatment group was slightly, but not significantly reduced. Metastasis was only detected in lung showing no effect by treatment with KP-10 (see chapter 3.5.3). Analysis was also carried out for mice without tumor cell injections and without treatment. Data of these control groups revealed low hDNA in CTCs respectively lung in one of the mice. This may be due to contaminations during the multi-step sample preparations including organ collection at study end, purification of each organ, shredding, DNA isolation and PCR analysis. Contaminations should be avoided by sterile working, but they still occurred. The potentially contaminated samples were not excluded from analysis to keep the analytical fault the same for all of the study groups.

Based on the results on tumor growth, KP-10 seems to be involved in antitumorigenic processes in vivo. This effect is discussed controversially in literature. In xenografts with prostate cancer cells, treatment with KP-10 led to reduced tumor volume [Cho et al., 2009b]. Whereas KP-10 decreased metastasis, but not tumor size in mouse models with melanoma [Ohtaki et al., 2001] or endometrial cancer cells [Kang et al., 2011]. In the present work, the reduced tumor growth was not expected. According to this, further investigations were carried out on antiproliferative properties of KP-10 in vitro. Results are discussed in chapter 4.2.3. An antimetastatic effect by KP-10 treatment was not observed for both breast cancer xenografts in the present work. Due to the results, this effect can not be excluded either. The metastatic spread was very inhomogeneous within the animal groups. Metastasis did not occur in any mouse and the extent of hDNA was diverse. To investigate the effect of a treatment with KP-10 on breast cancer metastasis, the used xenograft models are not valid enough. Enlargement of the study population or development of a more reproducible animal model may be two options to optimize the experimental setting. But the use of larger groups of animals is not arguable based on ethical reasons. The present results do not offer considerations on a possible use of KP-10 in therapy of the breast cancer disease.

\subsubsection{In vitro effects on proliferation}

The effect of KP-10 on tumor growth in vivo revealed lower proliferation rates in treated mice in the present work (see chapters 3.5.2 and 3.5.3). Research in literature demonstrated controversial results. A decreased effect on tumor proliferation was shown in xenografts with prostate cancer cells by treatment with KP-10 [Cho et al., 2009b]. Whereas KP-10 treatment in mouse models with melanoma [Ohtaki et al., 2001] respectively endometrial cancer cells led to decreased metastasis, but tumor growth was unaffected [Kang et al., 2011]. In vitro studies revealed no 
consistent data as well. In Chinese hamster ovary CHO cells transfected to overexpress GPR54, an antiproliferative effect was detected by treatment with kisspeptins [Hori et al., 2001, Kotani et al., 2001]. In addition, the same result was observed in murine fibroblasts NIH3T3 cells [Stafford et al., 2002] and MDA-MB-435s cells [Becker et al., 2005], which were overexpressing GPR54. No effect on cell proliferation was shown in carcinoma cell lines of pancreatic cancer [Masui et al., 2004] and renal cell cancer [Shoji et al., 2009], and in trophoblasts [Bilban et al., 2004]. All of these cells expressed GPR54 endogenously. In human umbilical vein endothelial cells HUVECs, a reduced proliferation was detected by KP-10 on the one hand [Ramaesh et al., 2010] and, on the other hand, no influence on proliferation was stated as well in these cells [Cho et al., 2009b]. Experiments with breast cancer cell lines revealed no influence on proliferation by treatment with KP-10, but expression of GPR54 was not studied [Cho et al., 2009a]. Taken together, cells were not affected in proliferation by KP-10, which showed endogenous GPR54 expression. Whereas an antiproliferative action of KP-10 was observed in cell models with artificial GPR54 expression. The aim of this part of the present work was to study the relationship of an antiproliferative effect of KP-10 in dependence of the nature of GPR54 expression. Conclusions for the inhibited tumor growth observed in vivo could be offered possibly by this analysis. Therefore, breast cancer cell lines were investigated in their GPR54 expression and proliferation under treatment with KP-10. Cells showing GPR54 overexpression were analyzed as well.

GPR54 expression levels were detected by immunocytochemistry, on mRNA and on protein levels. Based on controversial results in literature, three independent analytical assays were carried out. Immunocytochemical staining showed receptor expression in all of the nine breast cancer cell lines. GPR54 mRNA was found in T47D, ZR75-1 and MCF-7 cells. In MDA-MB-231, MDA-MB-435s, MDA-MB-453, HCC 70, HCC 1806 and HCC 1937 cells, no definite signal was received. All of the cell lines showed GPR54 protein expression in different amounts (see chapter 3.6.1.1). In literature, the cell lines T47D, ZR75-1 and MCF-7 were detected as GPR54-positive by mRNA analysis, whereas no receptor expression was found in MDA-MB-231 and MDA-MB-435 [Marot et al., 2007]. In contrast, GPR54 expression was shown in MDA-MB-231 cells by mRNA analysis [Martin et al., 2005, Pampillo et al., 2009]. In addition, mRNA analysis revealed no GPR54 in MDA-MB-435 cells [Becker et al., 2005, Nash et al., 2007]. According to the results of the present work showing GPR54 expression in all of the tested cell lines by immunocytochemical and protein expression analysis, the cell lines were regarded as receptor positive.

Proliferation was investigated in MDA-MB-231, MDA-MB-435s, HCC 1806 and MCF-7. These cell lines were chosen based on different GPR54 protein amounts. The results of the present work indicated no effect on proliferation in cells endogenously expressing GPR54 by KP-10 treatment. For proliferation studies in cells transfected to overexpress GPR54, B35 neuronal rat cells with stable mGPR54 expression were used. The amino acid structure of human GPR54 and murine GPR54 was shown to be homologous up to $82 \%$ [Kirby et al., 2010]. In addition, the gene 
products of human KISS1 and murine KISS1 were analyzed as related structures with a strong conservation within the active short forms [Stafford et al., 2002]. Human respectively murine receptor and peptides were used interchangeable in literature showing similar effects [Kotani et al., 2001, Mikkelsen et al., 2009]. Thus, it is acceptable to compare the results of breast cancer cells with endogenous hGPR54 expression with a mGPR54 transfected cell system.

Different B35 mGPR54 clones were used. They showed variable GPR54 expression levels by mRNA and protein analysis (see chapter 3.6.1.2). Proliferation was significantly inhibited by KP-10 in two of the clones. These results support the assumption, that KP-10 affects proliferation only in cells with artificial GPR54 expression. This may be due to the cellular GPR54 amount. On mRNA levels, comparison of receptor expression in the breast cancer and B35 cells revealed more mRNA within the transfected cells. Further experiments on protein levels could not confirm this observation. An explanation may be, that the antigen structure recognized by the used antibody was not represented comparably in hGPR54 respectively mGPR54. When experiments were run, an antibody was not available for a parallel detection of human and murine receptor. Nevertheless, the results of the present work match well with data in literature, that the breast cancer cell line MDA-MB-435s was not affected on proliferation by KP-10, but its GPR54 transfected clone was inhibited [Becker et al., 2005]. Evidence is given, that an antiproliferative effect of KP-10 is GPR54 mediated and only detectable in GPR54 overexpressing cell systems and not in cells with endogenous receptor expression.

Comparing the in vitro results of KP-10 on cell proliferation with the in vivo results on tumor growth, KP-10 seemed to reduce tumor size not directly on the cellular level. Another possible role of KP-10 in decreasing in vivo tumor growth may be by inhibition of angiogenetic mechanisms. In literature, studies with HUVECs demonstrated reduced tumor angiogenesis by KP-10 mediated suppression of VEGF expression [Cho et al., 2009b]. KP-10 was shown to decrease new vessel sprouting as important process in angiogenesis [Ramaesh et al., 2010]. Thus, further experiments are necessary, which investigate an interaction of KP-10 within angiogenesis in breast cancer xenografts. 


\section{Summary and conclusions}

The aim of the present work was to study cocultivated human breast cancer cells on invasive behavior and expression of genes involved in cell motility processes. Kisspeptin-10, a peptide derived from the metastasis suppressor gene KISS1, was shown to interfere within this cell system by inhibition of invasion. An influence of KP-10 in vivo should be further investigated to evaluate KP-10 as a possible therapeutic option in the metastatic breast cancer disease.

Breast cancer cell lines were classified phenotypically for their epithelial respectively mesenchymal properties by morphology and gene expression of motility markers. The individual invasion rates correlated well with the phenotypic characteristics demonstrating low cell movement for epithelial-like cells and high invasion for mesenchymal-like cells. Cocultivation with osteoblastlike osteosarcoma cells led to increased invasiveness in epithelial-like cells. The "bone"-directed cell movement was proved for invasion processes in three-dimensional settings. For migration, no influence of the cocultivation was observed. Microarray gene expression analysis indicated an involvement of EMT processes in the increased cellular invasiveness. This was based on enhanced mesenchymal properties, but without a change in the epithelial phenotype. Thus, a kind of "incomplete" EMT could have happened. In addition, the microarray data documented another important factor, CXCL12. Its upregulation could have triggered the coculture effect by an autocrine CXCL12/CXCR4 loop. Detection of inflammatory processes during coculture invasion revealed factors as EGF and interferons, which could have mediated the interaction of osteosarcoma cells and breast cancer cells. The present analysis indicated several starting points for further studies on the processes happening in the coculture system.

KP-10 was shown to decrease invasion in vitro by interaction with its receptor GPR54 and it reduced metastasis in vivo in different cancer cell systems. In a breast cancer xenograft, treatment with the peptide inhibited tumor growth. Further studies on cell proliferation revealed no antiproliferative effect in cells expressing GPR54 endogenously. Thus, KP-10 may be involved in angiogenetic processes in vivo, which represent an interesting assumption for future investigations. A conclusion could not be offered on an antimetastatic effect of KP-10 as possible therapeutic option in breast cancer based on the poor metastasis model in mice. 


\section{Bibliography}

R. C. Aguiar, Y. Yakushijin, S. Kharbanda, R. Salgia, J. A. Fletcher, and M. A. Shipp. Bal is a novel risk-related gene in diffuse large b-cell lymphomas that enhances cellular migration. Blood, 96(13): 4328-4334, Dec 2000.

C. Angelucci, G. Maulucci, G. Lama, G. Proietti, A. Colabianchi, M. Papi, A. Maiorana, M. D. Spirito, A. Micera, O. B. Balzamino, A. D. Leone, R. Masetti, and G. Sica. Epithelial-stromal interactions in human breast cancer: effects on adhesion, plasma membrane fluidity and migration speed and directness. PLoS One, 7(12):e50804, 2012.

M. A. Batzer and P. L. Deininger. Alu repeats and human genomic diversity. Nat Rev Genet, 3(5):370-9, 2002.

J. A. Becker, J. F. Mirjolet, J. Bernard, E. Burgeon, M. J. Simons, G. Vassart, M. Parmentier, and F. Libert. Activation of gpr54 promotes cell cycle arrest and apoptosis of human tumor cells through a specific transcriptional program not shared by other gq-coupled receptors. Biochem Biophys Res Commun, 326(3):677-86, 2005.

E. Bertran, L. Caja, E. Navarro, P. Sancho, J. Mainez, M. M. Murillo, A. Vinyals, A. Fabra, and I. Fabregat. Role of cxcr4/sdf-1 alpha in the migratory phenotype of hepatoma cells that have undergone epithelialmesenchymal transition in response to the transforming growth factor-beta. Cell Signal, 21(11):15951606, Nov 2009.

F.-C. Bidard, J.-Y. Pierga, A. Vincent-Salomon, and M.-F. Poupon. A "class action" against the microenvironment: do cancer cells cooperate in metastasis? Cancer Metastasis Rev, 27(1):5-10, Mar 2008.

M. Bilban, N. Ghaffari-Tabrizi, E. Hintermann, S. Bauer, S. Molzer, C. Zoratti, R. Malli, A. Sharabi, U. Hiden, W. Graier, M. Knofler, F. Andreae, O. Wagner, V. Quaranta, and G. Desoye. Kisspeptin-10, a kiss-1/metastin-derived decapeptide, is a physiological invasion inhibitor of primary human trophoblasts. J Cell Sci, 117(Pt 8):1319-28, 2004.

A. Billiau, V. G. Edy, H. Heremans, J. V. Damme, J. Desmyter, J. A. Georgiades, and P. D. Somer. Human interferon: mass production in a newly established cell line, mg-63. Antimicrob Agents Chemother, 12 (1):11-15, Jul 1977.

M. M. Bradford. A rapid and sensitive method for the quantitation of microgram quantities of protein utilizing the principle of protein-dye binding. Anal Biochem, 72:248-254, May 1976.

K. A. Brown, M. E. Aakre, A. E. Gorska, J. O. Price, S. E. Eltom, J. A. Pietenpol, and H. L. Moses. Induction by transforming growth factor-beta1 of epithelial to mesenchymal transition is a rare event in vitro. Breast Cancer Res, 6(3):R215-R231, 2004.

R. Cailleau, R. Young, M. Olivé, and W. J. Reeves. Breast tumor cell lines from pleural effusions. J Natl Cancer Inst, 53(3):661-674, Sep 1974.

R. Cailleau, M. Olivé, and Q. V. Cruciger. Long-term human breast carcinoma cell lines of metastatic origin: preliminary characterization. In Vitro, 14(11):911-915, Nov 1978. 
L. Castillo-Pichardo, M. M. Martínez-Montemayor, J. E. Martínez, K. M. Wall, L. A. Cubano, and S. Dharmawardhane. Inhibition of mammary tumor growth and metastases to bone and liver by dietary grape polyphenols. Clin Exp Metastasis, 26(6):505-516, 2009.

J. Y. Chai, C. Modak, W. Mouazzen, R. Narvaez, and J. Pham. Epithelial or mesenchymal: Where to draw the line? Biosci Trends, 4(3):130-142, Jun 2010.

A. F. Chambers. Mda-mb-435 and m14 cell lines: identical but not m14 melanoma? Cancer Res, 69 (13):5292-5293, Jul 2009.

E. Charafe-Jauffret, C. Ginestier, F. Monville, P. Finetti, J. Adélä̈de, N. Cervera, S. Fekairi, L. Xerri, J. Jacquemier, D. Birnbaum, and F. Bertucci. Gene expression profiling of breast cell lines identifies potential new basal markers. Oncogene, 25(15):2273-2284, Apr 2006.

J. Chen, D. Shi, X. Liu, S. Fang, J. Zhang, and Y. Zhao. Targeting sparc by lentivirus-mediated rna interference inhibits cervical cancer cell growth and metastasis. BMC Cancer, 12:464, 2012.

P.-S. Chen, M.-Y. Wang, S.-N. Wu, J.-L. Su, C.-C. Hong, S.-E. Chuang, M.-W. Chen, K.-T. Hua, Y.-L. Wu, S.-T. Cha, M. S. Babu, C.-N. Chen, P.-H. Lee, K.-J. Chang, and M.-L. Kuo. Ctgf enhances the motility of breast cancer cells via an integrin-alphavbeta3-erk1/2-dependent s100a4-upregulated pathway. J Cell Sci, 120(Pt 12):2053-2065, Jun 2007.

Y. Chen, M. V. Yusenko, and G. Kovacs. Lack of kiss1r expression is associated with rapid progression of conventional renal cell carcinomas. J Pathol, 223(1):46-53, 2011.

G. Z. Cheng, J. Chan, Q. Wang, W. Zhang, C. D. Sun, and L.-H. Wang. Twist transcriptionally upregulates akt2 in breast cancer cells leading to increased migration, invasion, and resistance to paclitaxel. Cancer Res, 67(5):1979-1987, Mar 2007.

S. G. Cho, D. Li, L. J. Stafford, J. Luo, M. Rodriguez-Villanueva, Y. Wang, and M. Liu. Kiss1 suppresses tnfalpha-induced breast cancer cell invasion via an inhibition of rhoa-mediated nf-kappab activation. $J$ Cell Biochem, 107(6):1139-1149, 2009a.

S. G. Cho, Z. Yi, X. Pang, T. Yi, Y. Wang, J. Luo, Z. Wu, D. Li, and M. Liu. Kisspeptin-10, a kiss1-derived decapeptide, inhibits tumor angiogenesis by suppressing sp1-mediated vegf expression and fak/rho gtpase activation. Cancer Res, 69(17):7062-70, 2009b.

P. Chomczynski and N. Sacchi. Single-step method of rna isolation by acid guanidinium thiocyanatephenol-chloroform extraction. Anal Biochem, 162(1):156-159, Apr 1987.

J. J. Christiansen and A. K. Rajasekaran. Reassessing epithelial to mesenchymal transition as a prerequisite for carcinoma invasion and metastasis. Cancer Res, 66(17):8319-8326, Sep 2006.

S. L. Chua, W. C. S. Too, B. Y. Khoo, and L. L. Few. Ubc and ywhaz as suitable reference genes for accurate normalisation of gene expression using mcf7, hct116 and hepg2 cell lines. Cytotechnology, 63 (6):645-654, Dec 2011.

M. K. Clements, T. P. McDonald, R. Wang, G. Xie, B. F. O’Dowd, S. R. George, C. P. Austin, and Q. Liu. Fmrfamide-related neuropeptides are agonists of the orphan g-protein-coupled receptor gpr54. Biochem Biophys Res Commun, 284(5):1189-93, 2001.

N. Conte, E. Charafe-Jauffret, B. Delaval, J. Adélaïde, C. Ginestier, J. Geneix, D. Isnardon, J. Jacquemier, and D. Birnbaum. Carcinogenesis and translational controls: Tacc1 is down-regulated in human cancers and associates with mrna regulators. Oncogene, 21(36):5619-5630, Aug 2002.

A. E. Curtis, J. H. Cooke, J. E. Baxter, J. R. Parkinson, A. S. Bataveljic, M. A. Ghatei, S. R. Bloom, and K. G. Murphy. A kisspeptin-10 analogue with greater in vivo bioactivity than kisspeptin-10. Am J Physiol Endocrinol Metab, 2009. 
K. M. Curtis, L. A. Gomez, C. Rios, E. Garbayo, A. P. Raval, M. A. Perez-Pinzon, and P. C. Schiller. Ef1alpha and rpl13a represent normalization genes suitable for $r$-qper analysis of bone marrow derived mesenchymal stem cells. BMC Mol Biol, 11:61, 2010.

X. Dai, Z. Mao, J. Huang, S. Xie, and H. Zhang. The cxcl12/cxcr4 autocrine loop increases the metastatic potential of non-small cell lung cancer in vitro. Oncol Lett, 5(1):277-282, Jan 2013.

G. Disibio and S. W. French. Metastatic patterns of cancers: results from a large autopsy study. Arch Pathol Lab Med, 132(6):931-939, Jun 2008.

DKG. Deutsche Krebsgesellschaft. DGGG. Deutsche Gesellschaft für Gynäkologische und Geburtshilfe. Leitlinienprogramm Onkologie. Interdisziplinäre S3-Leitlinie für die Diagnostik, Therpaie und Nachsorge des Mammakarzinoms. Berlin, 2012.

Y. Drabsch and P. T. Dijke. Tgf-beta signaling in breast cancer cell invasion and bone metastasis. J Mammary Gland Biol Neoplasia, 16(2):97-108, Jun 2011.

G. Ellison, T. Klinowska, R. F. Westwood, E. Docter, T. French, and J. C. Fox. Further evidence to support the melanocytic origin of mda-mb-435. Mol Pathol, 55(5):294-9, 2002.

L. W. Engel, N. A. Young, T. S. Tralka, M. E. Lippman, S. J. O’Brien, and M. J. Joyce. Establishment and characterization of three new continuous cell lines derived from human breast carcinomas. Cancer Res, 38(10):3352-3364, Oct 1978.

B. Fayard, F. Bianchi, J. Dey, E. Moreno, S. Djaffer, N. E. Hynes, and D. Monard. The serine protease inhibitor protease nexin-1 controls mammary cancer metastasis through Irp-1-mediated mmp-9 expression. Cancer Res, 69(14):5690-5698, Jul 2009.

N. Fenouille, M. Tichet, M. Dufies, A. Pottier, A. Mogha, J. K. Soo, S. Rocchi, A. Mallavialle, M.-D. Galibert, A. Khammari, J.-P. Lacour, R. Ballotti, M. Deckert, and S. Tartare-Deckert. The epithelialmesenchymal transition (emt) regulatory factor slug (snai2) is a downstream target of sparc and akt in promoting melanoma cell invasion. PLoS One, 7(7):e40378, 2012.

J. Ferlay, H.-R. Shin, F. Bray, D. Forman, C. Mathers, and D. M. Parkin. Estimates of worldwide burden of cancer in 2008: Globocan 2008. Int J Cancer, 127(12):2893-2917, Dec 2010.

E. Ferreira and M. J. Cronjé. Selection of suitable reference genes for quantitative real-time pcr in apoptosis-induced mcf-7 breast cancer cells. Mol Biotechnol, 50(2):121-128, Feb 2012.

I. J. Fidler. The pathogenesis of cancer metastasis: the 'seed and soil' hypothesis revisited. Nat Rev Cancer, 3(6):453-8, 2003.

P. Friedl and K. Wolf. Plasticity of cell migration: a multiscale tuning model. J Cell Biol, 188(1):11-19, Jan 2010.

C. Föst, F. Duwe, M. Hellriegel, S. Schweyer, G. Emons, and C. Gründker. Targeted chemotherapy for triple-negative breast cancers via Ihrh receptor. Oncol Rep, 25(5):1481-1487, May 2011.

A. F. Gazdar, V. Kurvari, A. Virmani, L. Gollahon, M. Sakaguchi, M. Westerfield, D. Kodagoda, V. Stasny, H. T. Cunningham, I. I. Wistuba, G. Tomlinson, V. Tonk, R. Ashfaq, A. M. Leitch, J. D. Minna, and J. W. Shay. Characterization of paired tumor and non-tumor cell lines established from patients with breast cancer. Int J Cancer, 78(6):766-774, Dec 1998.

F. Gergely, C. Karlsson, I. Still, J. Cowell, J. Kilmartin, and J. W. Raff. The tacc domain identifies a family of centrosomal proteins that can interact with microtubules. Proc Natl Acad Sci U S A, 97(26): 14352-14357, Dec 2000.

C. Glait-Santar, M. Pasmanik-Chor, V. Oron-Karni, and D. Benayahu. Molecular profiling of functional interactions between pre-osteoblastic and breast carcinoma cells. Genes Cells, 17(4):302-315, Apr 2012. 
A. Goldhirsch, W. C. Wood, A. S. Coates, R. D. Gelber, B. Thürlimann, H.-J. Senn, and P. members. Strategies for subtypes-dealing with the diversity of breast cancer: highlights of the st. gallen international expert consensus on the primary therapy of early breast cancer 2011. Ann Oncol, 22(8): 1736-1747, Aug 2011.

C. Greenwood, G. Metodieva, K. Al-Janabi, B. Lausen, L. Alldridge, L. Leng, R. Bucala, N. Fernandez, and M. V. Metodiev. Stat1 and cd74 overexpression is co-dependent and linked to increased invasion and lymph node metastasis in triple-negative breast cancer. J Proteomics, 75(10):3031-3040, Jun 2012.

M. J. Grimshaw, T. Hagemann, A. Ayhan, C. E. Gillett, C. Binder, and F. R. Balkwill. A role for endothelin-2 and its receptors in breast tumor cell invasion. Cancer Res, 64(7):2461-2468, Apr 2004.

E. Gutierrez-Pascual, J. Leprince, A. J. Martinez-Fuentes, I. Segalas-Milazzo, R. Pineda, J. Roa, M. Duran-Prado, L. Guilhaudis, E. Desperrois, A. Lebreton, L. Pinilla, M. C. Tonon, M. M. Malagon, H. Vaudry, M. Tena-Sempere, and J. P. Castano. In vivo and in vitro structure-activity relationships and structural conformation of kisspeptin-10-related peptides. Mol Pharmacol, 76(1):58-67, 2009.

J.-D. He, H.-L. Luo, J. Li, W.-T. Feng, and L.-B. Chen. Influences of the interferon induced transmembrane protein 1 on the proliferation, invasion, and metastasis of the colorectal cancer sw480 cell lines. Chin Med J (Engl), 125(3):517-522, Feb 2012.

R. Higuchi, C. Fockler, G. Dollinger, and R. Watson. Kinetic pcr analysis: real-time monitoring of dna amplification reactions. Biotechnology (N Y), 11(9):1026-1030, Sep 1993.

A. Hori, S. Honda, M. Asada, T. Ohtaki, K. Oda, T. Watanabe, Y. Shintani, T. Yamada, M. Suenaga, C. Kitada, H. Onda, T. Kurokawa, O. Nishimura, and M. Fujino. Metastin suppresses the motility and growth of cho cells transfected with its receptor. Biochem Biophys Res Commun, 286(5):958-63, 2001.

K. W. Hunter, N. P. S. Crawford, and J. Alsarraj. Mechanisms of metastasis. Breast Cancer Res, 10 Suppl 1:S2, 2008.

M. Ikeguchi, Y. Hirooka, and N. Kaibara. Quantitative reverse transcriptase polymerase chain reaction analysis for kiss-1 and orphan g-protein-coupled receptor (hot7t175) gene expression in hepatocellular carcinoma. J Cancer Res Clin Oncol, 129(9):531-5, 2003.

M. Ikeguchi, K. Yamaguchi, and N. Kaibara. Clinical significance of the loss of kiss-1 and orphan g-proteincoupled receptor (hot7t175) gene expression in esophageal squamous cell carcinoma. Clin Cancer Res, 10(4):1379-83, 2004.

M. Iwatsuki, K. Mimori, T. Yokobori, H. Ishi, T. Beppu, S. Nakamori, H. Baba, and M. Mori. Epithelialmesenchymal transition in cancer development and its clinical significance. Cancer Sci, 101(2):293-299, Feb 2010.

Y. Jiang, M. Berk, L. S. Singh, H. Tan, L. Yin, C. T. Powell, and Y. Xu. Kiss1 suppresses metastasis in human ovarian cancer via inhibition of protein kinase c alpha. Clin Exp Metastasis, 22(5):369-76, 2005.

J. A. Joyce and J. W. Pollard. Microenvironmental regulation of metastasis. Nat Rev Cancer, 9(4): 239-252, Apr 2009.

P. Juszczynski, J. L. Kutok, C. Li, J. Mitra, R. C. T. Aguiar, and M. A. Shipp. Bal1 and bbap are regulated by a gamma interferon-responsive bidirectional promoter and are overexpressed in diffuse large b-cell lymphomas with a prominent inflammatory infiltrate. Mol Cell Biol, 26(14):5348-5359, Jul 2006.

R. Kalluri and R. A. Weinberg. The basics of epithelial-mesenchymal transition. J Clin Invest, 119(6): 1420-8, 2009. 
H. Kang, R. E. Mansel, and W. G. Jiang. Genetic manipulation of stromal cell-derived factor-1 attests the pivotal role of the autocrine sdf-1-cxcr4 pathway in the aggressiveness of breast cancer cells. Int $J$ Oncol, 26(5):1429-1434, May 2005a.

H. Kang, G. Watkins, C. Parr, A. Douglas-Jones, R. E. Mansel, and W. G. Jiang. Stromal cell derived factor-1: its influence on invasiveness and migration of breast cancer cells in vitro, and its association with prognosis and survival in human breast cancer. Breast Cancer Res, 7(4):R402-R410, 2005b.

H. S. Kang, T. Baba, M. Mandai, N. Matsumura, J. Hamanishi, B. Kharma, E. Kondoh, Y. Yoshioka, S. Oishi, N. Fujii, S. K. Murphy, and I. Konishi. Gpr54 is a target for suppression of metastasis in endometrial cancer. Mol Cancer Ther, 10(4):580-590, Apr 2011.

Y. Kang, P. M. Siegel, W. Shu, M. Drobnjak, S. M. Kakonen, C. Cordón-Cardo, T. A. Guise, and J. Massagué. A multigenic program mediating breast cancer metastasis to bone. Cancer Cell, 3(6): 537-549, Jun 2003.

M. Karin, T. Lawrence, and V. Nizet. Innate immunity gone awry: linking microbial infections to chronic inflammation and cancer. Cell, 124(4):823-835, Feb 2006.

I. Keydar, L. Chen, S. Karby, F. R. Weiss, J. Delarea, M. Radu, S. Chaitcik, and H. J. Brenner. Establishment and characterization of a cell line of human breast carcinoma origin. Eur $J$ Cancer, 15(5): 659-670, May 1979.

H. R. Kirby, J. J. Maguire, W. H. Colledge, and A. P. Davenport. International union of basic and clinical pharmacology. Ixxvii. kisspeptin receptor nomenclature, distribution, and function. Pharmacol Rev, 62 (4):565-78, 2010.

C. A. Klein. Parallel progression of primary tumours and metastases. Nat Rev Cancer, 9(4):302-312, Apr 2009.

T. Kobayashi, S. Sasaki, N. Tomita, S. Fukui, N. Kuroda, M. Nakayama, A. Kiba, Y. Takatsu, T. Ohtaki, F. Itoh, and A. Baba. Synthesis and structure-activity relationships of 2-acylamino-4,6-diphenylpyridine derivatives as novel antagonists of gpr54. Bioorg Med Chem, 18(11):3841-3859, Jun 2010.

M. I. Kokkinos, R. Wafai, M. K. Wong, D. F. Newgreen, E. W. Thompson, and M. Waltham. Vimentin and epithelial-mesenchymal transition in human breast cancer-observations in vitro and in vivo. Cells Tissues Organs, 185(1-3):191-203, 2007.

M. Kotani, M. Detheux, A. Vandenbogaerde, D. Communi, J. M. Vanderwinden, E. Le Poul, S. Brezillon, R. Tyldesley, N. Suarez-Huerta, F. Vandeput, C. Blanpain, S. N. Schiffmann, G. Vassart, and M. Parmentier. The metastasis suppressor gene kiss-1 encodes kisspeptins, the natural ligands of the orphan g protein-coupled receptor gpr54. J Biol Chem, 276(37):34631-6, 2001.

W. Kuohung, M. Burnett, D. Mukhtyar, E. Schuman, J. Ni, W. F. Crowley, M. A. Glicksman, and U. B. Kaiser. A high-throughput small-molecule ligand screen targeted to agonists and antagonists of the g-protein-coupled receptor gpr54. J Biomol Screen, 15(5):508-517, Jun 2010.

U. K. Laemmli. Cleavage of structural proteins during the assembly of the head of bacteriophage $t 4$. Nature, 227(5259):680-5, 1970.

S. C. Lebret, D. F. Newgreen, E. W. Thompson, and M. L. Ackland. Induction of epithelial to mesenchymal transition in pmc42-la human breast carcinoma cells by carcinoma-associated fibroblast secreted factors. Breast Cancer Res, 9(1):R19, 2007.

J. H. Lee and D. R. Welch. Identification of highly expressed genes in metastasis-suppressed chromosome $6 /$ human malignant melanoma hybrid cells using subtractive hybridization and differential display. Int J Cancer, 71(6):1035-44, 1997a. 
J. H. Lee and D. R. Welch. Suppression of metastasis in human breast carcinoma mda-mb-435 cells after transfection with the metastasis suppressor gene, kiss-1. Cancer Res, 57(12):2384-7, 1997b.

J. H. Lee, M. E. Miele, D. J. Hicks, K. K. Phillips, J. M. Trent, B. E. Weissman, and D. R. Welch. Kiss-1, a novel human malignant melanoma metastasis-suppressor gene. J Natl Cancer Inst, 88(23):1731-7, 1996.

J. Y. Lee, J. S. Moon, Y. J. Eu, C. W. Lee, S. T. Yang, S. K. Lee, H. H. Jung, H. H. Kim, H. Rhim, J. Y. Seong, and J. I. Kim. Molecular interaction between kisspeptin decapeptide analogs and a lipid membrane. Arch Biochem Biophys, 485(2):109-14, 2009.

N. Li, H.-X. Wang, J. Zhang, Y.-P. Ye, and G.-Y. He. Kiss-1 inhibits the proliferation and invasion of gastric carcinoma cells. World J Gastroenterol, 18(15):1827-1833, Apr 2012.

K. J. Livak, S. J. Flood, J. Marmaro, W. Giusti, and K. Deetz. Oligonucleotides with fluorescent dyes at opposite ends provide a quenched probe system useful for detecting pcr product and nucleic acid hybridization. PCR Methods Appl, 4(6):357-362, Jun 1995.

A. Mantovani, B. Savino, M. Locati, L. Zammataro, P. Allavena, and R. Bonecchi. The chemokine system in cancer biology and therapy. Cytokine Growth Factor Rev, 21(1):27-39, Feb 2010.

D. Marot, I. Bieche, C. Aumas, S. Esselin, C. Bouquet, S. Vacher, G. Lazennec, M. Perricaudet, F. Kuttenn, R. Lidereau, and N. de Roux. High tumoral levels of kiss1 and g-protein-coupled receptor 54 expression are correlated with poor prognosis of estrogen receptor-positive breast tumors. Endocr Relat Cancer, 14(3):691-702, 2007.

T. A. Martin, G. Watkins, and W. G. Jiang. Kiss-1 expression in human breast cancer. Clin Exp Metastasis, 22(6):503-11, 2005.

T. Masui, R. Doi, T. Mori, E. Toyoda, M. Koizumi, K. Kami, D. Ito, S. C. Peiper, J. R. Broach, S. Oishi, A. Niida, N. Fujii, and M. Imamura. Metastin and its variant forms suppress migration of pancreatic cancer cells. Biochem Biophys Res Commun, 315(1):85-92, 2004.

L. Mathot and J. Stenninger. Behavior of seeds and soil in the mechanism of metastasis: a deeper understanding. Cancer Sci, 103(4):626-631, Apr 2012.

B. A. McKenzie, A. Barrieux, and N. M. Varki. A novel detection system for submicroscopic human metastases in athymic mice. Cancer Commun, 3(1):15-19, Jan 1991.

L. R. McNally, D. R. Welch, B. H. Beck, L. J. Stafford, J. W. Long, J. C. Sellers, Z. Q. Huang, W. E. Grizzle, C. R. Stockard, K. T. Nash, and D. J. Buchsbaum. Kiss1 over-expression suppresses metastasis of pancreatic adenocarcinoma in a xenograft mouse model. Clin Exp Metastasis, 27(8):591-600, Dec 2010.

J. A. Merritt, D. M. Meltzer, L. A. Ball, and E. C. Borden. 2-5a synthetase activity in patients with metastatic carcinoma and its response to interferon treatment. Prog Clin Biol Res, 202:423-430, 1985.

M. E. Miele, G. Robertson, J. H. Lee, A. Coleman, C. T. McGary, P. B. Fisher, T. G. Lugo, and D. R. Welch. Metastasis suppressed, but tumorigenicity and local invasiveness unaffected, in the human melanoma cell line meljuso after introduction of human chromosomes 1 or 6 . Mol Carcinog, 15(4): 284-99, 1996.

J. D. Mikkelsen, A. H. Bentsen, L. Ansel, V. Simonneaux, and A. Juul. Comparison of the effects of peripherally administered kisspeptins. Regul Pept, 152(1-3):95-100, 2009.

V. Mirisola, A. Zuccarino, B. E. Bachmeier, M. P. Sormani, J. Falter, A. Nerlich, and U. Pfeffer. Cxcl12/sdf1 expression by breast cancers is an independent prognostic marker of disease-free and overall survival. Eur J Cancer, 45(14):2579-2587, Sep 2009. 
A. Müller, B. Homey, H. Soto, N. Ge, D. Catron, M. E. Buchanan, T. McClanahan, E. Murphy, W. Yuan, S. N. Wagner, J. L. Barrera, A. Mohar, E. Verástegui, and A. Zlotnik. Involvement of chemokine receptors in breast cancer metastasis. Nature, 410(6824):50-56, Mar 2001.

S. Mooez, F. A. Malik, M. A. Kayani, R. Rashid, A. Zahid, and A. Khan. Expressional alterations and transcript isoforms of metastasis suppressor genes (kai1 and kiss1) in breast cancer patients. Asian Pac J Cancer Prev, 12(10):2785-2791, 2011.

A. I. Muir, L. Chamberlain, N. A. Elshourbagy, D. Michalovich, D. J. Moore, A. Calamari, P. G. Szekeres, H. M. Sarau, J. K. Chambers, P. Murdock, K. Steplewski, U. Shabon, J. E. Miller, S. E. Middleton, J. G. Darker, C. G. Larminie, S. Wilson, D. J. Bergsma, P. Emson, R. Faull, K. L. Philpott, and D. C. Harrison. Axor12, a novel human g protein-coupled receptor, activated by the peptide kiss-1. J Biol Chem, 276(31):28969-75, 2001.

K. Mullis, F. Faloona, S. Scharf, R. Saiki, G. Horn, and H. Erlich. Specific enzymatic amplification of dna in vitro: the polymerase chain reaction. Cold Spring Harb Symp Quant Biol, 51 Pt 1:263-273, 1986.

J. R. Munoz, B. R. Stoutenger, A. P. Robinson, J. L. Spees, and D. J. Prockop. Human stem/progenitor cells from bone marrow promote neurogenesis of endogenous neural stem cells in the hippocampus of mice. Proc Natl Acad Sci U S A, 102(50):18171-18176, Dec 2005.

K. Nagai, R. Doi, F. Katagiri, T. Ito, A. Kida, M. Koizumi, T. Masui, Y. Kawaguchi, K. Tomita, S. Oishi, N. Fujii, and S. Uemoto. Prognostic value of metastin expression in human pancreatic cancer. J Exp Clin Cancer Res, 28:9, 2009.

K. T. Nash, P. A. Phadke, J. M. Navenot, D. R. Hurst, M. A. Accavitti-Loper, E. Sztul, K. S. Vaidya, A. R. Frost, J. C. Kappes, S. C. Peiper, and D. R. Welch. Requirement of kiss 1 secretion for multiple organ metastasis suppression and maintenance of tumor dormancy. J Natl Cancer Inst, 99(4):309-21, 2007.

J. M. Navenot, Z. Wang, M. Chopin, N. Fujii, and S. C. Peiper. Kisspeptin-10-induced signaling of gpr54 negatively regulates chemotactic responses mediated by cxcr4: a potential mechanism for the metastasis suppressor activity of kisspeptins. Cancer Res, 65(22):10450-6, 2005.

J. M. Navenot, N. Fujii, and S. C. Peiper. Activation of rho and rho-associated kinase by gpr54 and kiss1 metastasis suppressor gene product induces changes of cell morphology and contributes to apoptosis. Mol Pharmacol, 75(6):1300-6, 2009.

R. M. Neve, K. Chin, J. Fridlyand, J. Yeh, F. L. Baehner, T. Fevr, L. Clark, N. Bayani, J. P. Coppe, F. Tong, T. Speed, P. T. Spellman, S. DeVries, A. Lapuk, N. J. Wang, W. L. Kuo, J. L. Stilwell, D. Pinkel, D. G. Albertson, F. M. Waldman, F. McCormick, R. B. Dickson, M. D. Johnson, M. Lippman, S. Ethier, A. Gazdar, and J. W. Gray. A collection of breast cancer cell lines for the study of functionally distinct cancer subtypes. Cancer Cell, 10(6):515-27, 2006.

A. Niida, Z. Wang, K. Tomita, S. Oishi, H. Tamamura, A. Otaka, J. M. Navenot, J. R. Broach, S. C. Peiper, and N. Fujii. Design and synthesis of downsized metastin (45-54) analogs with maintenance of high gpr54 agonistic activity. Bioorg Med Chem Lett, 16(1):134-7, 2006.

T. Ohtaki, Y. Shintani, S. Honda, H. Matsumoto, A. Hori, K. Kanehashi, Y. Terao, S. Kumano, Y. Takatsu, Y. Masuda, Y. Ishibashi, T. Watanabe, M. Asada, T. Yamada, M. Suenaga, C. Kitada, S. Usuki, T. Kurokawa, H. Onda, O. Nishimura, and M. Fujino. Metastasis suppressor gene kiss-1 encodes peptide ligand of a g-protein-coupled receptor. Nature, 411(6837):613-7, 2001.

T. Olbrich. Einfluss von Kisspeptin-10 auf die knochengerichtete Migration und Invasion von Mammakarzinomzellen. Dissertation, 2010a.

T. Olbrich, E. Ziegler, G. Türk, A. Schubert, G. Emons, and C. Gründker. Kisspeptin-10 inhibits bonedirected migration of gpr54-positive breast cancer cells: Evidence for a dose-window effect. Gynecol Oncol, 119(3):571-578, Dec 2010b. 
T. Onoue, D. Uchida, N. M. Begum, Y. Tomizuka, H. Yoshida, and M. Sato. Epithelial-mesenchymal transition induced by the stromal cell-derived factor-1/cxcr4 system in oral squamous cell carcinoma cells. Int J Oncol, 29(5):1133-8, 2006.

M. J. Orsini, M. A. Klein, M. P. Beavers, P. J. Connolly, S. A. Middleton, and K. H. Mayo. Metastin (kiss-1) mimetics identified from peptide structure-activity relationship-derived pharmacophores and directed small molecule database screening. J Med Chem, 50(3):462-71, 2007.

S. Paget. The distribution of secondary growths in cancer of the breast. 1889. Cancer Metastasis Rev, 8 (2):98-101, Aug 1889.

M. Pampillo, N. Camuso, J. E. Taylor, J. M. Szereszewski, M. R. Ahow, M. Zajac, R. P. Millar, M. Bhattacharya, and A. V. Babwah. Regulation of gpr54 signaling by grk2 and beta-arrestin. Mol Endocrinol, 23(12):2060-74, 2009.

K. Pantel and R. H. Brakenhoff. Dissecting the metastatic cascade. Nat Rev Cancer, 4(6):448-456, Jun 2004.

M. Parri and P. Chiarugi. Rac and rho gtpases in cancer cell motility control. Cell Commun Signal, 8:23, 2010.

L. M. Prentice, C. Klausen, S. Kalloger, M. Köbel, S. McKinney, J. L. Santos, C. Kenney, E. Mehl, C. B. Gilks, P. Leung, K. Swenerton, D. G. Huntsman, and S. A. J. Aparicio. Kisspeptin and gpr54 immunoreactivity in a cohort of 518 patients defines favourable prognosis and clear cell subtype in ovarian carcinoma. BMC Med, 5:33, 2007.

J. E. Price and R. D. Zhang. Studies of human breast cancer metastasis using nude mice. Cancer Metastasis Rev, 8(4):285-297, Feb 1990.

S. E. Quaggin, G. B. V. Heuvel, and P. Igarashi. Pod-1, a mesoderm-specific basic-helix-loop-helix protein expressed in mesenchymal and glomerular epithelial cells in the developing kidney. Mech Dev, 71(1-2): 37-48, Feb 1998.

J. M. Rae, S. J. Ramus, M. Waltham, J. E. Armes, I. G. Campbell, R. Clarke, R. J. Barndt, M. D. Johnson, and E. W. Thompson. Common origins of mda-mb-435 cells from various sources with those shown to have melanoma properties. Clin Exp Metastasis, 21(6):543-552, 2004.

T. Ramaesh, J. J. Logie, A. K. Roseweir, R. P. Millar, B. R. Walker, P. W. F. Hadoke, and R. M. Reynolds. Kisspeptin-10 inhibits angiogenesis in human placental vessels ex vivo and endothelial cells in vitro. Endocrinology, 151(12):5927-5934, Dec 2010.

M. M. Richert, K. S. Vaidya, C. N. Mills, D. Wong, W. Korz, D. R. Hurst, and D. R. Welch. Inhibition of cxcr4 by ctce-9908 inhibits breast cancer metastasis to lung and bone. Oncol Rep, 21(3):761-767, Mar 2009.

E. Richtig, G. Langmann, K. Mullner, G. Richtig, and J. Smolle. Calculated tumour volume as a prognostic parameter for survival in choroidal melanomas. Eye (Lond), 18(6):619-23, 2004.

RKI. Robert Koch-Institut. Krebs in Deutschland 2007/2008. Berlin, 8th edition, 2012.

A. K. Roseweir, A. S. Kauffman, J. T. Smith, K. A. Guerriero, K. Morgan, J. Pielecka-Fortuna, R. Pineda, M. L. Gottsch, M. Tena-Sempere, S. M. Moenter, E. Terasawa, I. J. Clarke, R. A. Steiner, and R. P. Millar. Discovery of potent kisspeptin antagonists delineate physiological mechanisms of gonadotropin regulation. J Neurosci, 29(12):3920-9, 2009.

A. K. Roseweir, A. A. Katz, and R. P. Millar. Kisspeptin-10 inhibits cell migration in vitro via a receptorgsk3 beta-fak feedback loop in htr8svneo cells. Placenta, 33(5):408-415, May 2012.

D. D. Ross, J. E. Karp, T. T. Chen, and L. A. Doyle. Expression of breast cancer resistance protein in blast cells from patients with acute leukemia. Blood, 96(1):365-368, Jul 2000. 
Z. J. Rutnam and B. B. Yang. The non-coding 3' utr of cd44 induces metastasis by regulating extracellular matrix functions. J Cell Sci, 125(Pt 8):2075-2085, Apr 2012.

A. Sasaki, B. F. Boyce, B. Story, K. R. Wright, M. Chapman, R. Boyce, G. R. Mundy, and T. Yoneda. Bisphosphonate risedronate reduces metastatic human breast cancer burden in bone in nude mice. Cancer Res, 55(16):3551-3557, Aug 1995.

T. Schneider, F. Osl, T. Friess, H. Stockinger, and W. V. Scheuer. Quantification of human alu sequences by real-time pcr-an improved method to measure therapeutic efficacy of anti-metastatic drugs in human xenotransplants. Clin Exp Metastasis, 19(7):571-582, 2002.

A. Schubert, T. Hawighorst, G. Emons, and C. Gründker. Agonists and antagonists of gnrh-i and -ii reduce metastasis formation by triple-negative human breast cancer cells in vivo. Breast Cancer Res Treat, 130(3):783-790, Dec 2011.

D. Schubert, S. Heinemann, W. Carlisle, H. Tarikas, B. Kimes, J. Patrick, J. H. Steinbach, W. Culp, and B. L. Brandt. Clonal cell lines from the rat central nervous system. Nature, 249(454):224-227, May 1974.

C. J. Scotton, J. L. Wilson, D. Milliken, G. Stamp, and F. R. Balkwill. Epithelial cancer cell migration: a role for chemokine receptors? Cancer Res, 61(13):4961-4965, Jul 2001.

S. Sellappan, R. Grijalva, X. Zhou, W. Yang, M. B. Eli, G. B. Mills, and D. Yu. Lineage infidelity of mda-mb-435 cells: expression of melanocyte proteins in a breast cancer cell line. Cancer Res, 64(10): 3479-3485, May 2004.

X. Shen, S. Wang, H. Wang, M. Liang, L. Xiao, and Z. Wang. The role of sdf-1/cxcr4 axis in ovarian cancer metastasis. J Huazhong Univ Sci Technolog Med Sci, 29(3):363-367, Jun 2009.

C. Sheridan, H. Kishimoto, R. K. Fuchs, S. Mehrotra, P. Bhat-Nakshatri, C. H. Turner, J. Goulet, R., S. Badve, and H. Nakshatri. Cd44+/cd24- breast cancer cells exhibit enhanced invasive properties: an early step necessary for metastasis. Breast Cancer Res, 8(5):R59, 2006.

R. H. Shoemaker, A. M. Smythe, L. Wu, M. S. Balaschak, and M. R. Boyd. Evaluation of metastatic human tumor burden and response to therapy in a nude mouse xenograft model using a molecular probe for repetitive human dna sequences. Cancer Res, 52(10):2791-2796, May 1992.

S. Shoji, X. Y. Tang, S. Umemura, J. Itoh, S. Takekoshi, M. Shima, Y. Usui, Y. Nagata, T. Uchida, R. Y. Osamura, and T. Terachi. Metastin inhibits migration and invasion of renal cell carcinoma with overexpression of metastin receptor. Eur Urol, 55(2):441-9, 2009.

S. Singh, U. P. Singh, W. E. Grizzle, and J. W. Lillard. Cxcl12-cxcr4 interactions modulate prostate cancer cell migration, metalloproteinase expression and invasion. Lab Invest, 84(12):1666-1676, Dec 2004.

C. L. Sommers, S. W. Byers, E. W. Thompson, J. A. Torri, and E. P. Gelmann. Differentiation state and invasiveness of human breast cancer cell lines. Breast Cancer Res Treat, 31(2-3):325-35, 1994.

H. D. Soule, J. Vazguez, A. Long, S. Albert, and M. Brennan. A human cell line from a pleural effusion derived from a breast carcinoma. J Natl Cancer Inst, 51(5):1409-1416, Nov 1973.

L. J. Stafford, C. Xia, W. Ma, Y. Cai, and M. Liu. Identification and characterization of mouse metastasissuppressor kiss1 and its g-protein-coupled receptor. Cancer Res, 62(19):5399-404, 2002.

J. M. Szereszewski, M. Pampillo, M. R. Ahow, S. Offermanns, M. Bhattacharya, and A. V. Babwah. Gpr54 regulates erk1/2 activity and hypothalamic gene expression in a $\mathrm{g}$ ?(q/11) and ?-arrestin-dependent manner. PLoS One, 5(9):e12964, 2010. 
M. Taki, K. Higashikawa, S. Yoneda, S. Ono, H. Shigeishi, M. Nagayama, and N. Kamata. Up-regulation of stromal cell-derived factor-1alpha and its receptor cxcr4 expression accompanied with epithelialmesenchymal transition in human oral squamous cell carcinoma. Oncol Rep, 19(4):993-8, 2008.

T. Takino, N. Koshikawa, H. Miyamori, M. Tanaka, T. Sasaki, Y. Okada, M. Seiki, and H. Sato. Cleavage of metastasis suppressor gene product kiss-1 protein/metastin by matrix metalloproteinases. Oncogene, 22(30):4617-26, 2003.

M. A. Taylor, J. G. Parvani, and W. P. Schiemann. The pathophysiology of epithelial-mesenchymal transition induced by transforming growth factor-beta in normal and malignant mammary epithelial cells. J Mammary Gland Biol Neoplasia, 15(2):169-190, Jun 2010.

K. Tomita, A. Niida, S. Oishi, H. Ohno, J. Cluzeau, J. M. Navenot, Z. X. Wang, S. C. Peiper, and N. Fujii. Structure-activity relationship study on small peptidic gpr54 agonists. Bioorg Med Chem, 14 (22):7595-603, 2006.

K. Tomita, T. Narumi, A. Niida, S. Oishi, H. Ohno, and N. Fujii. Fmoc-based solid-phase synthesis of gpr54-agonistic pentapeptide derivatives containing alkene- and fluoroalkene-dipeptide isosteres. Biopolymers, 88(2):272-8, 2007.

K. Tomita, S. Oishi, H. Ohno, S. C. Peiper, and N. Fujii. Development of novel g-protein-coupled receptor 54 agonists with resistance to degradation by matrix metalloproteinase. J Med Chem, 51(23):7645-9, 2008.

S. Tyagi and F. R. Kramer. Molecular beacons: probes that fluoresce upon hybridization. Nat Biotechnol, 14(3):303-308, Mar 1996.

D. Uchida, N. M. Begum, A. Almofti, K. ichi Nakashiro, H. Kawamata, Y. Tateishi, H. Hamakawa, H. Yoshida, and M. Sato. Possible role of stromal-cell-derived factor-1/cxcr4 signaling on lymph node metastasis of oral squamous cell carcinoma. Exp Cell Res, 290(2):289-302, Nov 2003.

D. Uchida, T. Onoue, Y. Tomizuka, N. M. Begum, Y. Miwa, H. Yoshida, and M. Sato. Involvement of an autocrine stromal cell derived factor-1/cxcr4 system on the distant metastasis of human oral squamous cell carcinoma. Mol Cancer Res, 5(7):685-694, Jul 2007.

M. Uchino, H. Kojima, K. Wada, M. Imada, F. Onoda, H. Satofuka, T. Utsugi, and Y. Murakami. Nuclear beta-catenin and cd44 upregulation characterize invasive cell populations in non-aggressive mcf-7 breast cancer cells. BMC Cancer, 10:414, 2010.

S. Valastyan and R. A. Weinberg. Tumor metastasis: molecular insights and evolving paradigms. Cell, 147(2):275-292, Oct 2011.

F. van Zijl, G. Krupitza, and W. Mikulits. Initial steps of metastasis: cell invasion and endothelial transmigration. Mutat Res, 728(1-2):23-34, 2011.

I. M. Verma, R. A. Firtel, H. F. Lodish, and D. Baltimore. Synthesis of dna complementary to cellular slime mold messenger rna by reverse transcriptase. Biochemistry, 13(19):3917-3922, Sep 1974.

U. Veronesi, P. Boyle, A. Goldhirsch, R. Orecchia, and G. Viale. Breast cancer. Lancet, 365(9472): 1727-1741, 2005.

L. D. Volk-Draper, S. Rajput, K. L. Hall, A. Wilber, and S. Ran. Novel model for basaloid triple-negative breast cancer: behavior in vivo and response to therapy. Neoplasia, 14(10):926-942, Oct 2012.

J. von Alten, S. Fister, H. Schulz, V. Viereck, K.-H. Frosch, G. Emons, and C. Gründker. Gnrh analogs reduce invasiveness of human breast cancer cells. Breast Cancer Res Treat, 100(1):13-21, Nov 2006.

S. L. Voytik-Harbin, A. O. Brightman, B. Waisner, C. H. Lamar, and S. F. Badylak. Application and evaluation of the alamarblue assay for cell growth and survival of fibroblasts. In Vitro Cell Dev Biol Anim, 34(3):239-46, 1998. 
J. Wang, R. Loberg, and R. S. Taichman. The pivotal role of cxcl12 (sdf-1)/cxcr4 axis in bone metastasis. Cancer Metastasis Rev, 25(4):573-587, Dec 2006.

D. R. Welch, P. Chen, M. E. Miele, C. T. McGary, J. M. Bower, E. J. Stanbridge, and B. E. Weissman. Microcell-mediated transfer of chromosome 6 into metastatic human c8161 melanoma cells suppresses metastasis but does not inhibit tumorigenicity. Oncogene, 9(1):255-62, 1994.

WHO. World Health Organization. IARC Handbooks of Cancer Prevention. Breast Cancer Screening., volume 7. IARC Press, Lyon, 2002.

WHO. World Health Organization. Classification of Tumours. Pathology and Genetics of Tumours of the Breast and Female Genital Organs. IARC Press, Lyon, 2003.

Y. Wu and B. P. Zhou. New insights of epithelial-mesenchymal transition in cancer metastasis. Acta Biochim Biophys Sin (Shanghai), 40(7):643-50, 2008.

C. Yan, H. Wang, and D. D. Boyd. Kiss-1 represses 92-kda type iv collagenase expression by downregulating $n f-k a p p a b$ binding to the promoter as a consequence of ikappa balpha -induced block of p65/p50 nuclear translocation. J Biol Chem, 276(2):1164-72, 2001.

M. Yilmaz and G. Christofori. Mechanisms of motility in metastasizing cells. Mol Cancer Res, 8(5): 629-642, May 2010.

T. Yoneda. Cellular and molecular basis of preferential metastasis of breast cancer to bone. J Orthop Sci, 5(1):75-81, 2000.

K. Yoshioka, Y. Ohno, Y. Horiguchi, C. Ozu, K. Namiki, and M. Tachibana. Effects of a kiss-1 peptide, a metastasis suppressor gene, on the invasive ability of renal cell carcinoma cells through a modulation of a matrix metalloproteinase 2 expression. Life Sci, 83(9-10):332-338, Aug 2008.

D. A. Zajchowski, M. F. Bartholdi, Y. Gong, L. Webster, H. L. Liu, A. Munishkin, C. Beauheim, S. Harvey, S. P. Ethier, and P. H. Johnson. Identification of gene expression profiles that predict the aggressive behavior of breast cancer cells. Cancer Res, 61(13):5168-5178, Jul 2001.

J. Zavadil and E. P. Bottinger. Tgf-beta and epithelial-to-mesenchymal transitions. Oncogene, 24(37): 5764-74, 2005.

E. Ziegler, T. Olbrich, G. Emons, and C. Gründker. Antiproliferative effects of kisspeptin-10 depend on artificial gpr54 (kiss1r) expression levels. Oncol Rep, 29(2):549-554, Feb 2013. 
Appendix 
A1 Sample heatmap of the microarray data

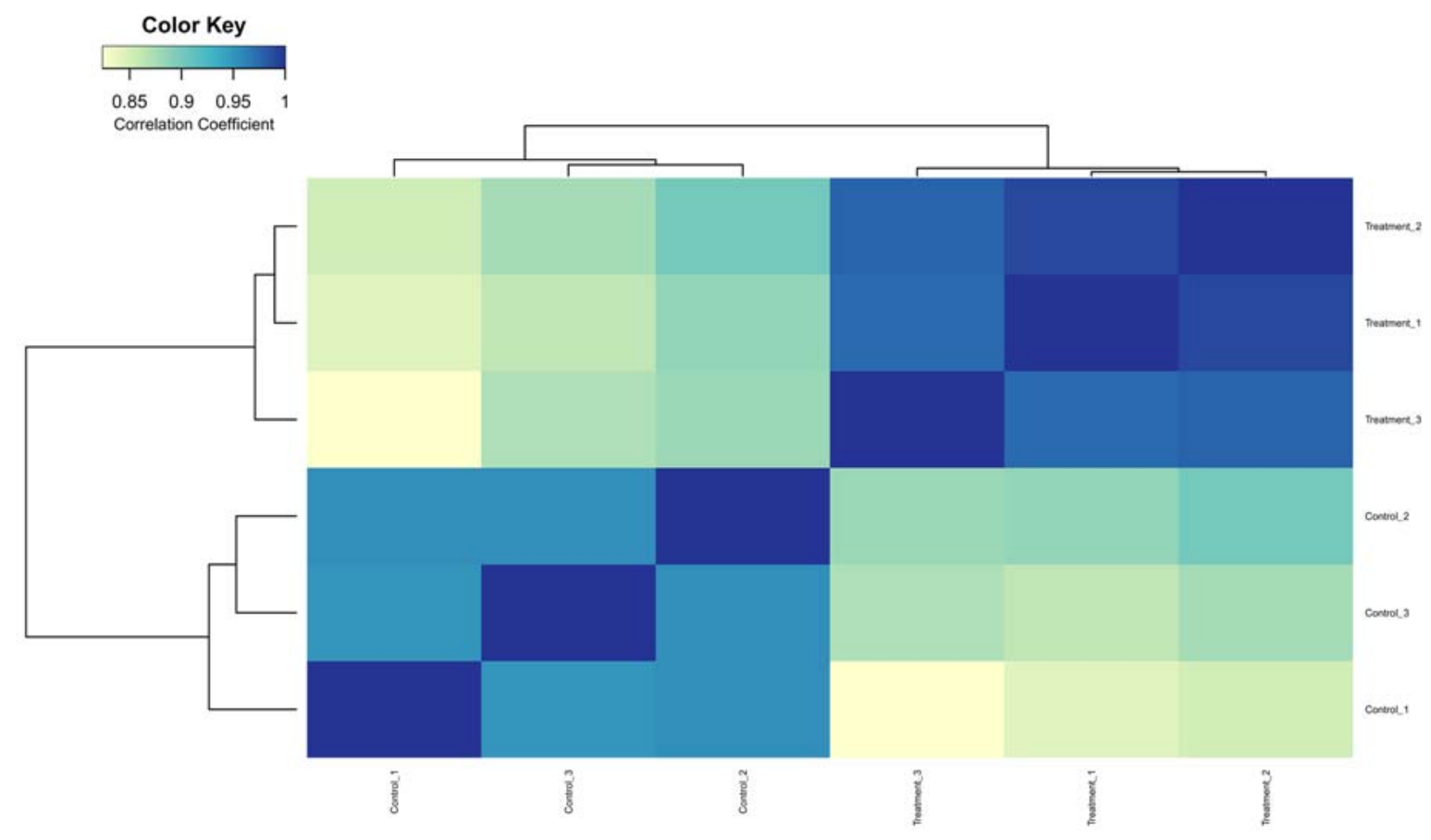




\section{A2 Candidates list of the microarray data}

\begin{tabular}{|c|c|c|c|c|c|c|c|}
\hline Symbol & Description & Probe & Gene & $\begin{array}{l}\text { logFC } \\
\text { Treatment- } \\
\text { Control }\end{array}$ & $\begin{array}{l}\text { AveExpr } \\
\text { Treatment- } \\
\text { Control }\end{array}$ & $\begin{array}{l}\text { P.Value } \\
\text { Treatment- } \\
\text { Control }\end{array}$ & $\begin{array}{l}\text { FDR } \\
\text { Treatment- } \\
\text { Control }\end{array}$ \\
\hline KYNU & kynureninase & 8942 at & 8942 & $-2,85$ & 7,406266074 & $1,16 \mathrm{E}-04$ & $0,68 \%$ \\
\hline KITLG & KIT ligand & 4254_at & 4254 & $-2,72$ & 8,475037516 & $2,88 \mathrm{E}-06$ & $0,10 \%$ \\
\hline ERBB4 & $\begin{array}{l}\text { v-erb-a erythroblastic } \\
\text { leukemia viral oncogene } \\
\text { homolog } 4 \text { (avian) }\end{array}$ & 2066_at & 2066 & $-2,56$ & 8,447631597 & $1,36 \mathrm{E}-06$ & $0,08 \%$ \\
\hline NRCAM & $\begin{array}{l}\text { neuronal cell adhesion } \\
\text { molecule }\end{array}$ & 4897_at & 4897 & $-2,48$ & 7,828567861 & $4,50 \mathrm{E}-06$ & $0,12 \%$ \\
\hline EFEMP1 & $\begin{array}{l}\text { EGF containing fibulin-like } \\
\text { extracellular matrix protein } 1\end{array}$ & 2202_at & 2202 & $-2,38$ & 8,919480102 & $5,98 \mathrm{E}-05$ & $0,47 \%$ \\
\hline TACC1 & $\begin{array}{l}\text { transforming, acidic } \\
\text { coiled-coil containing protein } \\
1\end{array}$ & 6867_at & 6867 & $-2,17$ & 9,548784414 & $1,68 \mathrm{E}-05$ & $0,22 \%$ \\
\hline NPNT & nephronectin & 255743_at & 255743 & $-2,13$ & 10,44608789 & 9,73E-07 & $0,07 \%$ \\
\hline LIN7A & lin-7 homolog A (C. elegans) & 8825 at & 8825 & $-2,10$ & 7,696356695 & $8,90 \mathrm{E}-06$ & $0,16 \%$ \\
\hline UPK1A & uroplakin $1 \mathrm{~A}$ & 11045 at & 11045 & $-2,04$ & 7,583522905 & $1,33 \mathrm{E}-03$ & $2,71 \%$ \\
\hline SALL4 & sal-like 4 (Drosophila) & 57167 at & 57167 & $-2,02$ & 8,174255164 & $1,25 \mathrm{E}-04$ & $0,71 \%$ \\
\hline TRAV13-2 & $\begin{array}{l}\text { T cell receptor al pha variable } \\
13-2\end{array}$ & 28670 at & 28670 & $-1,98$ & 6,28037951 & $2,35 \mathrm{E}-03$ & $3,63 \%$ \\
\hline ST8SIA4 & $\begin{array}{l}\text { ST8 } \\
\text { alpha-N-acetyl-neuraminide } \\
\text { alpha-2,8-sialyltransferase } 4\end{array}$ & 7903 at & 7903 & $-1,89$ & 7,204850359 & $1,40 \mathrm{E}-06$ & $0,08 \%$ \\
\hline PMP22 & peripheral myelin protein 22 & 5376 at & 5376 & $-1,88$ & 8,679818574 & $1,70 \mathrm{E}-04$ & $0,86 \%$ \\
\hline OR4K2 & $\begin{array}{l}\text { olfactory receptor, family } 4 \text {, } \\
\text { subfamily } K, \text { member } 2\end{array}$ & 390431_at & 390431 & $-1,87$ & 7,144559138 & $1,91 \mathrm{E}-03$ & $3,20 \%$ \\
\hline IGFBP5 & $\begin{array}{l}\text { insulin-like growth factor } \\
\text { binding protein } 5\end{array}$ & 3488 at & 3488 & $-1,83$ & 7,380510353 & $2,60 \mathrm{E}-03$ & $3,82 \%$ \\
\hline GDPD3 & $\begin{array}{l}\text { glycerophosphodiester } \\
\text { phosphodiesterase domain } \\
\text { containing } 3\end{array}$ & 79153 at & 79153 & $-1,81$ & 9,116905382 & $8,95 \mathrm{E}-04$ & $2,16 \%$ \\
\hline FAM83B & $\begin{array}{l}\text { family with sequence } \\
\text { similarity } 83, \text { member } B\end{array}$ & 222584 at & 222584 & $-1,78$ & 10,07170838 & $4,60 \mathrm{E}-07$ & $0,04 \%$ \\
\hline MIR181B1 & microRNA 181b-1 & 406955_at & 406955 & $-1,77$ & 8,874114332 & $6,18 \mathrm{E}-07$ & $0,05 \%$ \\
\hline CMYA5 & cardiomyopathy associated 5 & 202333_at & 202333 & $-1,74$ & 6,911259143 & $4,65 \mathrm{E}-04$ & $1,49 \%$ \\
\hline TRAV14DV4 & $\begin{array}{l}T \text { cell receptor al pha variable } \\
14 / \text { delta variable } 4\end{array}$ & 28669 at & 28669 & $-1,72$ & 4,545292137 & 3,93E-06 & $0,11 \%$ \\
\hline NELL2 & NEL-like 2 (chicken) & 4753_at & 4753 & $-1,70$ & 5,592997192 & $3,73 \mathrm{E}-05$ & $0,36 \%$ \\
\hline PGM5 & phosphoglucomutase 5 & 5239_at & 5239 & $-1,67$ & 6,092060241 & $5,91 \mathrm{E}-05$ & $0,47 \%$ \\
\hline SLC26A2 & $\begin{array}{l}\text { solute carrier family } 26 \\
\text { (sulfate transporter), } \\
\text { member } 2\end{array}$ & 1836 at & 1836 & $-1,64$ & 8,090917908 & $2,16 \mathrm{E}-06$ & $0,09 \%$ \\
\hline GSTM3 & $\begin{array}{l}\text { glutathione S-transferase mu } \\
3 \text { (brain) }\end{array}$ & 2947_at & 2947 & $-1,61$ & 8,180511204 & $1,02 \mathrm{E}-05$ & $0,17 \%$ \\
\hline LIFR & $\begin{array}{l}\text { leukemia inhibitory factor } \\
\text { receptor alpha }\end{array}$ & 3977_at & 3977 & $-1,59$ & 6,879524731 & $1,29 \mathrm{E}-05$ & $0,20 \%$ \\
\hline MATN2 & matrilin 2 & 4147_at & 4147 & $-1,54$ & 8,065115075 & $6,60 \mathrm{E}-05$ & $0,50 \%$ \\
\hline MKRN9P & $\begin{array}{l}\text { makorin ring finger protein } \\
9, \text { pseudogene }\end{array}$ & 400058_at & 400058 & $-1,51$ & 6,777580298 & $2,22 \mathrm{E}-03$ & $3,49 \%$ \\
\hline ABCG2 & $\begin{array}{l}\text { ATP-binding cassette, } \\
\text { sub-family G (WHITE), } \\
\text { member } 2\end{array}$ & 9429_at & 9429 & $-1,49$ & 5,943967309 & $2,76 \mathrm{E}-04$ & $1,14 \%$ \\
\hline NEDD4L & $\begin{array}{l}\text { neural precursor cell } \\
\text { expressed, developmentally } \\
\text { down-regulated 4-like }\end{array}$ & 23327 at & 23327 & $-1,48$ & 8,679391458 & 1,67E-05 & $0,22 \%$ \\
\hline OR51A4 & $\begin{array}{l}\text { olfactory receptor, family } 51 \text {, } \\
\text { subfamily A, member } 4\end{array}$ & 401666_at & 401666 & $-1,48$ & 5,622793421 & $2,15 \mathrm{E}-03$ & $3,44 \%$ \\
\hline SLC6A14 & $\begin{array}{l}\text { solute carrier family } 6 \\
\text { (amino acid transporter), } \\
\text { member } 14\end{array}$ & 11254 at & 11254 & $-1,48$ & 7,454409733 & $1,21 \mathrm{E}-04$ & $0,70 \%$ \\
\hline
\end{tabular}




\begin{tabular}{|c|c|c|c|c|c|c|c|}
\hline Symbol & Description & Probe & Gene & $\begin{array}{l}\log \mathrm{FC} \\
\text { Treatment- } \\
\text { Control }\end{array}$ & $\begin{array}{l}\text { AveExpr } \\
\text { Treatment- } \\
\text { Control }\end{array}$ & $\begin{array}{l}\text { P.Value } \\
\text { Treatment- } \\
\text { Control }\end{array}$ & $\begin{array}{l}\text { FDR } \\
\text { Treatment- } \\
\text { Control }\end{array}$ \\
\hline C18orf1 & $\begin{array}{l}\text { chromosome } 18 \text { open } \\
\text { reading frame } 1\end{array}$ & 753 at & 753 & $-1,47$ & 7,543478928 & $2,77 E-06$ & $0,10 \%$ \\
\hline OR2M5 & $\begin{array}{l}\text { olfactory receptor, family } 2 \text {, } \\
\text { subfamily } M, \text { member } 5\end{array}$ & 127059_at & 127059 & $-1,46$ & 6,199568116 & $3,94 \mathrm{E}-03$ & $4,80 \%$ \\
\hline ANXA3 & annexin $A 3$ & 306 at & 306 & $-1,42$ & 9,192114952 & $2,97 \mathrm{E}-04$ & $1,19 \%$ \\
\hline EFNA1 & ephrin-A1 & 1942 at & 1942 & $-1,39$ & 8,554657267 & $6,01 \mathrm{E}-06$ & $0,13 \%$ \\
\hline IL1R1 & interleukin 1 receptor, type I & 3554_at & 3554 & $-1,39$ & 5,553848659 & $5,46 \mathrm{E}-05$ & $0,44 \%$ \\
\hline FAM190A & $\begin{array}{l}\text { family with sequence } \\
\text { similarity } 190, \text { member A }\end{array}$ & 401145 at & 401145 & $-1,38$ & 6,560566784 & $3,46 \mathrm{E}-06$ & $0,10 \%$ \\
\hline MMP16 & $\begin{array}{l}\text { matrix metallopeptidase } 16 \\
\text { (membrane-inserted) }\end{array}$ & 4325 at & 4325 & $-1,37$ & 8,496755098 & $1,05 \mathrm{E}-06$ & $0,07 \%$ \\
\hline GPR115 & $\begin{array}{l}\text { G protein-coupled receptor } \\
115\end{array}$ & 221393_at & 221393 & $-1,37$ & 5,070890942 & $1,68 \mathrm{E}-03$ & $2,98 \%$ \\
\hline ODZ3 & $\begin{array}{l}\text { odz, odd } \mathrm{Oz} / \text { ten-m homolog } \\
3 \text { (Drosophila) }\end{array}$ & 55714_at & 55714 & $-1,36$ & 7,544591964 & $2,38 \mathrm{E}-04$ & $1,04 \%$ \\
\hline YPEL3 & yippee-like 3 (Drosophila) & 83719 at & 83719 & $-1,35$ & 7,598959105 & $2,62 \mathrm{E}-05$ & $0,30 \%$ \\
\hline PMEPA1 & $\begin{array}{l}\text { prostate transmembrane } \\
\text { protein, androgen induced } 1\end{array}$ & 56937_at & 56937 & $-1,34$ & 7,604917876 & $5,89 \mathrm{E}-04$ & $1,73 \%$ \\
\hline DCDC2 & $\begin{array}{l}\text { doublecortin domain } \\
\text { containing } 2\end{array}$ & 51473_at & 51473 & $-1,34$ & 8,290499171 & $1,87 \mathrm{E}-03$ & $3,17 \%$ \\
\hline OR2M3 & $\begin{array}{l}\text { olfactory receptor, family } 2 \text {, } \\
\text { subfamily } M, \text { member } 3\end{array}$ & 127062 at & 127062 & $-1,33$ & 5,071130007 & $5,83 \mathrm{E}-04$ & $1,73 \%$ \\
\hline BMF & Bcl2 modifying factor & 90427 at & 90427 & $-1,32$ & 7,006350766 & $2,00 \mathrm{E}-05$ & $0,25 \%$ \\
\hline HEBP1 & heme binding protein 1 & 50865_at & 50865 & $-1,32$ & 9,182310955 & $2,68 \mathrm{E}-04$ & $1,12 \%$ \\
\hline CRIM1 & $\begin{array}{l}\text { cysteine rich transmembrane } \\
\text { BMP regulator } 1 \\
\text { (chordin-like) }\end{array}$ & 51232 at & 51232 & $-1,32$ & 8,467742946 & $2,13 \mathrm{E}-03$ & $3,43 \%$ \\
\hline OR2M2 & $\begin{array}{l}\text { olfactory receptor, family } 2 \text {, } \\
\text { subfamily } M, \text { member } 2\end{array}$ & 391194 at & 391194 & $-1,31$ & 4,761045367 & $1,92 \mathrm{E}-03$ & $3,21 \%$ \\
\hline OR4K5 & $\begin{array}{l}\text { olfactory receptor, family } 4 \text {, } \\
\text { subfamily } \mathrm{K}, \text { member } 5\end{array}$ & 79317 at & 79317 & $-1,31$ & 5,914293006 & $4,25 \mathrm{E}-03$ & $4,99 \%$ \\
\hline UTRN & utrophin & 7402 at & 7402 & $-1,30$ & 7,433529373 & $1,10 \mathrm{E}-04$ & $0,67 \%$ \\
\hline YPEL2 & yippee-like 2 (Drosophila) & 388403 at & 388403 & $-1,30$ & 7,918533368 & $1,61 \mathrm{E}-04$ & $0,84 \%$ \\
\hline SMYD2 & $\begin{array}{l}\text { SET and MYND domain } \\
\text { containing } 2\end{array}$ & 56950_at & 56950 & $-1,29$ & 8,162206929 & $7,30 \mathrm{E}-04$ & $1,96 \%$ \\
\hline KLHL24 & kelch-like 24 (Drosophila) & 54800_at & 54800 & $-1,29$ & 7,967952863 & $1,24 \mathrm{E}-03$ & $2,59 \%$ \\
\hline MIR181A1 & microRNA 181a-1 & 406995_at & 406995 & $-1,28$ & 5,032751235 & $6,19 \mathrm{E}-06$ & $0,13 \%$ \\
\hline SLC25A30 & $\begin{array}{l}\text { solute carrier family } 25 \text {, } \\
\text { member } 30\end{array}$ & 253512 at & 253512 & $-1,27$ & 7,698576039 & $1,11 \mathrm{E}-04$ & $0,67 \%$ \\
\hline WWC2 & $\begin{array}{l}\text { WW and C2 domain } \\
\text { containing } 2\end{array}$ & 80014_at & 80014 & $-1,26$ & 7,690531912 & $5,53 \mathrm{E}-05$ & $0,44 \%$ \\
\hline IRF6 & interferon regulatory factor 6 & 3664_at & 3664 & $-1,26$ & 8,214889991 & $7,49 \mathrm{E}-04$ & $1,99 \%$ \\
\hline OR4M1 & $\begin{array}{l}\text { olfactory receptor, family } 4 \text {, } \\
\text { subfamily } M, \text { member } 1\end{array}$ & 441670 at & 441670 & $-1,25$ & 5,249881964 & $1,95 \mathrm{E}-03$ & $3,24 \%$ \\
\hline OR14J1 & $\begin{array}{l}\text { olfactory receptor, family } 14 \text {, } \\
\text { subfamily } \mathrm{J}, \text { member } 1\end{array}$ & 442191_at & 442191 & $-1,24$ & 5,535000971 & $2,76 \mathrm{E}-03$ & $3,94 \%$ \\
\hline PLXDC2 & plexin domain containing 2 & 84898_at & 84898 & $-1,24$ & 7,894429179 & $4,89 \mathrm{E}-05$ & $0,42 \%$ \\
\hline $\begin{array}{l}\text { STON1- } \\
\text { GTF2A1L }\end{array}$ & $\begin{array}{l}\text { STON1-GTF2A1L } \\
\text { readth rough }\end{array}$ & 286749 at & 286749 & $-1,23$ & 4,40048753 & $4,50 \mathrm{E}-04$ & $1,46 \%$ \\
\hline RAB31 & $\begin{array}{l}\text { RAB31, member RAS } \\
\text { oncogene family }\end{array}$ & 11031_at & 11031 & $-1,22$ & 8,94421261 & $3,16 \mathrm{E}-05$ & $0,33 \%$ \\
\hline MIR27B & microRNA 27b & 407019 at & 407019 & $-1,22$ & 5,640110131 & $3,37 \mathrm{E}-04$ & $1,27 \%$ \\
\hline TM4SF18 & $\begin{array}{l}\text { transmembrane } 4 \mathrm{~L} \text { six } \\
\text { family member } 18\end{array}$ & 116441_at & 116441 & $-1,21$ & 6,112117913 & $3,45 \mathrm{E}-03$ & $4,46 \%$ \\
\hline BLNK & B-cell linker & 29760 at & 29760 & $-1,20$ & 6,937868872 & $3,36 \mathrm{E}-04$ & $1,27 \%$ \\
\hline MIR15A & microRNA 15a & 406948 at & 406948 & $-1,18$ & 7,240130056 & $6,59 \mathrm{E}-05$ & $0,50 \%$ \\
\hline C3orf79 & $\begin{array}{l}\text { chromosome } 3 \text { open reading } \\
\text { frame } 79\end{array}$ & 152118 at & 152118 & $-1,18$ & 5,177150289 & $2,16 \mathrm{E}-04$ & $0,99 \%$ \\
\hline PMEL & premelanosome protein & $6490 \_$at & 6490 & $-1,17$ & 6,273951385 & $9,37 \mathrm{E}-05$ & $0,60 \%$ \\
\hline SECTM1 & $\begin{array}{l}\text { secreted and transmembrane } \\
1\end{array}$ & 6398 at & 6398 & $-1,17$ & 7,756533126 & $8,27 E-04$ & $2,11 \%$ \\
\hline
\end{tabular}




\begin{tabular}{|c|c|c|c|c|c|c|c|}
\hline Symbol & Description & Probe & Gene & $\begin{array}{l}\text { logFC } \\
\text { Treatment- } \\
\text { Control }\end{array}$ & $\begin{array}{l}\text { AveExpr } \\
\text { Treatment- } \\
\text { Control }\end{array}$ & $\begin{array}{l}\text { P.Value } \\
\text { Treatment- } \\
\text { Control }\end{array}$ & $\begin{array}{l}\text { FDR } \\
\text { Treatment- } \\
\text { Control }\end{array}$ \\
\hline EPB41L5 & $\begin{array}{l}\text { erythrocyte membrane } \\
\text { protein band } 4.1 \text { like } 5\end{array}$ & 57669 at & 57669 & $-1,17$ & 9,742491922 & $1,23 E-05$ & $0,19 \%$ \\
\hline DCAF4 & $\begin{array}{l}\text { DDB1 and CUL4 associated } \\
\text { factor } 4\end{array}$ & 26094 at & 26094 & $-1,17$ & 9,413860834 & $5,16 \mathrm{E}-04$ & $1,61 \%$ \\
\hline CCNG2 & cyclin G2 & 901 at & 901 & $-1,17$ & 10,37886412 & $1,27 \mathrm{E}-03$ & $2,63 \%$ \\
\hline MXRA5 & $\begin{array}{l}\text { matrix-remodelling } \\
\text { associated } 5\end{array}$ & 25878 at & 25878 & $-1,17$ & 8,265112689 & $2,36 \mathrm{E}-04$ & $1,04 \%$ \\
\hline MIR30C2 & microRNA 30c-2 & 407032 at & 407032 & $-1,15$ & 6,130575775 & $2,35 \mathrm{E}-03$ & $3,63 \%$ \\
\hline ATP7B & $\begin{array}{l}\text { ATPase, } \mathrm{Cu}++ \text { transporting, } \\
\text { beta polypeptide }\end{array}$ & 540 at & 540 & $-1,14$ & 7,25354421 & $8,56 \mathrm{E}-05$ & $0,57 \%$ \\
\hline MAGI1 & $\begin{array}{l}\text { membrane associated } \\
\text { guanylate kinase, WW and } \\
\text { PDZ domain containing } 1\end{array}$ & 9223_at & 9223 & $-1,14$ & 9,028643182 & $9,12 \mathrm{E}-05$ & $0,59 \%$ \\
\hline C7orf41 & $\begin{array}{l}\text { chromosome } 7 \text { open reading } \\
\text { frame } 41\end{array}$ & 222166 at & 222166 & $-1,13$ & 6,596552875 & $2,70 \mathrm{E}-06$ & $0,10 \%$ \\
\hline CYP4B1 & $\begin{array}{l}\text { cytochrome P450, family } 4 \text {, } \\
\text { subfamily B, polypeptide } 1\end{array}$ & 1580_at & 1580 & $-1,13$ & 7,096721096 & $1,59 \mathrm{E}-03$ & $2,91 \%$ \\
\hline DIO1 & $\begin{array}{l}\text { deiodinase, iodothyronine, } \\
\text { type I }\end{array}$ & 1733_at & 1733 & $-1,13$ & 6,012958646 & $1,18 \mathrm{E}-04$ & $0,69 \%$ \\
\hline ZNF217 & zinc finger protein 217 & 7764_at & 7764 & $-1,13$ & 11,18543811 & $3,80 \mathrm{E}-05$ & $0,36 \%$ \\
\hline IL20 & interleukin 20 & 50604_at & 50604 & $-1,13$ & 7,996277607 & $1,52 \mathrm{E}-04$ & $0,82 \%$ \\
\hline SPRR2A & small proline-rich protein $2 \mathrm{~A}$ & 6700 at & 6700 & $-1,09$ & 5,619491707 & $1,57 \mathrm{E}-03$ & $2,91 \%$ \\
\hline ARHGEF37 & $\begin{array}{l}\text { Rho guanine nucleotide } \\
\text { exchange factor (GEF) } 37\end{array}$ & 389337_at & 389337 & $-1,09$ & 6,560581069 & $1,45 \mathrm{E}-05$ & $0,21 \%$ \\
\hline PLLP & plasmolipin & 51090 at & 51090 & $-1,09$ & 8,209628928 & $1,71 \mathrm{E}-05$ & $0,23 \%$ \\
\hline EDIL3 & $\begin{array}{l}\text { EGF-like repeats and } \\
\text { discoidin I-like domains } 3\end{array}$ & 10085 at & 10085 & $-1,07$ & 4,995220205 & $1,23 \mathrm{E}-03$ & $2,59 \%$ \\
\hline TPK1 & thiamin pyrophosphokinase 1 & $27010_{\text {at }}$ & 27010 & $-1,07$ & 6,051792774 & $2,15 \mathrm{E}-06$ & $0,09 \%$ \\
\hline SMPDL3A & $\begin{array}{l}\text { sphingomyelin } \\
\text { phosphodiesterase, acid-like } \\
\text { 3A }\end{array}$ & 10924_at & 10924 & $-1,07$ & 7,943882155 & $7,21 \mathrm{E}-05$ & $0,52 \%$ \\
\hline ATP8A1 & $\begin{array}{l}\text { ATPase, aminophospholipid } \\
\text { transporter (APLT), class I, } \\
\text { type } 8 \mathrm{~A} \text {, member } 1\end{array}$ & 10396_at & 10396 & $-1,07$ & 6,310351626 & $4,35 \mathrm{E}-05$ & $0,39 \%$ \\
\hline CTNND2 & $\begin{array}{l}\text { catenin (cadherin-associated } \\
\text { protein), delta } 2 \text { (neural } \\
\text { plakophilin-related } \\
\text { arm-repeat protein) }\end{array}$ & 1501_at & 1501 & $-1,06$ & 7,867247363 & $5,43 \mathrm{E}-04$ & $1,66 \%$ \\
\hline C6orf155 & $\begin{array}{l}\text { chromosome } 6 \text { open reading } \\
\text { frame } 155\end{array}$ & 79940 at & 79940 & $-1,05$ & 8,183079237 & $2,19 \mathrm{E}-05$ & $0,27 \%$ \\
\hline OR52N5 & $\begin{array}{l}\text { olfactory receptor, family } 52 \text {, } \\
\text { subfamily } \mathrm{N} \text {, member } 5\end{array}$ & 390075 at & 390075 & $-1,05$ & 5,538167564 & $3,04 \mathrm{E}-03$ & $4,16 \%$ \\
\hline PVALB & parvalbumin & 5816_at & 5816 & $-1,04$ & 7,644177408 & $3,78 \mathrm{E}-03$ & $4,67 \%$ \\
\hline HDGFRP3 & $\begin{array}{l}\text { hepatoma-derived growth } \\
\text { factor, related protein } 3\end{array}$ & 50810_at & 50810 & $-1,04$ & 9,414705288 & $5,51 \mathrm{E}-05$ & $0,44 \%$ \\
\hline INHBB & inhibin, beta B & 3625 at & 3625 & $-1,03$ & 8,947079417 & $1,50 \mathrm{E}-03$ & $2,84 \%$ \\
\hline AMIGO2 & $\begin{array}{l}\text { adhesion molecule with } \\
\text { lg-like domain } 2\end{array}$ & 347902_at & 347902 & $-1,03$ & 6,704993012 & $1,72 \mathrm{E}-04$ & $0,86 \%$ \\
\hline OR6N2 & $\begin{array}{l}\text { olfactory receptor, family } 6 \text {, } \\
\text { subfamily } N \text {, member } 2\end{array}$ & 81442 at & 81442 & $-1,02$ & 6,824105065 & $2,55 \mathrm{E}-03$ & $3,79 \%$ \\
\hline TACSTD2 & $\begin{array}{l}\text { tumor-associated calcium } \\
\text { signal transducer } 2\end{array}$ & 4070_at & 4070 & $-1,01$ & 10,51765811 & $9,73 \mathrm{E}-04$ & $2,28 \%$ \\
\hline C3orf52 & $\begin{array}{l}\text { chromosome } 3 \text { open reading } \\
\text { frame } 52\end{array}$ & 79669 at & 79669 & $-1,01$ & 6,850900692 & $1,34 \mathrm{E}-04$ & $0,75 \%$ \\
\hline FBP1 & $\begin{array}{l}\text { fructose-1,6-bisphosphatase } \\
1\end{array}$ & 2203_at & 2203 & $-1,01$ & 11,42804045 & $1,90 \mathrm{E}-06$ & $0,09 \%$ \\
\hline OR52N4 & $\begin{array}{l}\text { olfactory receptor, family } 52 \text {, } \\
\text { subfamily } N \text {, member } 4\end{array}$ & 390072_at & 390072 & $-1,01$ & 4,328490978 & $2,43 \mathrm{E}-03$ & $3,67 \%$ \\
\hline OR8K5 & $\begin{array}{l}\text { olfactory receptor, family } 8 \text {, } \\
\text { subfamily } K \text {, member } 5\end{array}$ & 219453_at & 219453 & $-1,00$ & 4,630598018 & $1,70 \mathrm{E}-03$ & $3,00 \%$ \\
\hline CNBD1 & $\begin{array}{l}\text { cyclic nucleotide binding } \\
\text { domain containing } 1\end{array}$ & 168975_at & 168975 & $-1,00$ & 5,312125451 & $1,63 \mathrm{E}-03$ & $2,94 \%$ \\
\hline
\end{tabular}




\begin{tabular}{|c|c|c|c|c|c|c|c|}
\hline Symbol & Description & Probe & Gene & $\begin{array}{l}\log \mathrm{FC} \\
\text { Treatment- } \\
\text { Control }\end{array}$ & $\begin{array}{l}\text { AveExpr } \\
\text { Treatment- } \\
\text { Control }\end{array}$ & $\begin{array}{l}\text { P.Value } \\
\text { Treatment- } \\
\text { Control }\end{array}$ & $\begin{array}{l}\text { FDR } \\
\text { Treatment- } \\
\text { Control }\end{array}$ \\
\hline SNTB1 & $\begin{array}{l}\text { syntrophin, beta } 1 \\
\text { (dystrophin-associated } \\
\text { protein } \mathrm{A} 1,59 \mathrm{kDa} \text {, basic } \\
\text { component } 1 \text { ) }\end{array}$ & 6641_at & 6641 & 1,00 & 7,187488838 & $1,63 \mathrm{E}-03$ & $2,94 \%$ \\
\hline PTGS1 & $\begin{array}{l}\text { prostaglandin-endoperoxide } \\
\text { synthase } 1 \text { (prostaglandin } \\
\mathrm{G} / \mathrm{H} \text { synthase and } \\
\text { cyclooxygenase) }\end{array}$ & 5742 at & 5742 & 1,00 & 6,369589517 & $1,99 \mathrm{E}-06$ & $0,09 \%$ \\
\hline FBXO39 & F-box protein 39 & 162517_at & 162517 & 1,00 & 7,054042868 & $8,14 \mathrm{E}-06$ & $0,15 \%$ \\
\hline EPAS1 & $\begin{array}{l}\text { endothelial PAS domain } \\
\text { protein } 1\end{array}$ & 2034_at & 2034 & 1,00 & 6,389630304 & $4,87 E-05$ & $0,42 \%$ \\
\hline EIF2AK2 & $\begin{array}{l}\text { eukaryotic translation } \\
\text { initiation factor } 2 \text {-alpha } \\
\text { kinase } 2\end{array}$ & 5610_at & 5610 & 1,01 & 11,20446581 & $7,30 \mathrm{E}-06$ & $0,14 \%$ \\
\hline AFF1 & $\begin{array}{l}\text { AF4/FMR2 family, member } \\
1\end{array}$ & 4299 at & 4299 & 1,02 & 9,062796128 & $8,43 E-06$ & $0,15 \%$ \\
\hline DOCK8 & dedicator of cytokinesis 8 & 81704_at & 81704 & 1,02 & 7,299326429 & $6,06 \mathrm{E}-06$ & $0,13 \%$ \\
\hline $\mathrm{HSH} 2 \mathrm{D}$ & $\begin{array}{l}\text { hematopoietic } \mathrm{SH} 2 \text { domain } \\
\text { containing }\end{array}$ & 84941 at & 84941 & 1,02 & 7,014823175 & $3,44 \mathrm{E}-05$ & $0,34 \%$ \\
\hline ME1 & $\begin{array}{l}\text { malic enzyme } 1, \\
\text { NADP }(+) \text {-dependent, } \\
\text { cytosolic }\end{array}$ & 4199 at & 4199 & 1,02 & 9,108477168 & $1,32 \mathrm{E}-05$ & $0,20 \%$ \\
\hline PRPS1 & $\begin{array}{l}\text { phosphoribosyl } \\
\text { pyrophosphate synthetase } 1\end{array}$ & 5631_at & 5631 & 1,02 & 8,017260516 & $4,02 \mathrm{E}-06$ & $0,11 \%$ \\
\hline OASL & $\begin{array}{l}\text { 2'-5'-oligoad enylate } \\
\text { synthetase-like }\end{array}$ & 8638_at & 8638 & 1,02 & 7,322763345 & $1,14 \mathrm{E}-04$ & $0,68 \%$ \\
\hline ELOVL6 & $\begin{array}{l}\text { ELOVL family member } 6 \text {, } \\
\text { elongation of long chain } \\
\text { fatty acids (FEN1/Elo2, } \\
\text { SUR4/Elo3-like, yeast) }\end{array}$ & 79071 at & 79071 & 1,03 & 8,69090685 & $3,48 \mathrm{E}-04$ & $1,28 \%$ \\
\hline IRS1 & insulin receptor substrate 1 & 3667 at & 3667 & 1,03 & 7,027577498 & $6,56 \mathrm{E}-06$ & $0,13 \%$ \\
\hline PDCD4 & $\begin{array}{l}\text { programmed cell death } 4 \\
\text { (neoplastic transformation } \\
\text { inhibitor) }\end{array}$ & 27250 at & 27250 & 1,03 & 9,715621762 & $7,00 \mathrm{E}-05$ & $0,51 \%$ \\
\hline ETS2 & $\begin{array}{l}\text { v-ets erythroblastosis virus } \\
\text { E26 oncogene homolog } 2 \\
\text { (avian) }\end{array}$ & 2114 at & 2114 & 1,03 & 8,139213327 & $1,70 \mathrm{E}-04$ & $0,86 \%$ \\
\hline FMN1 & formin 1 & 342184_at & 342184 & 1,03 & 7,097489435 & $2,41 \mathrm{E}-03$ & $3,66 \%$ \\
\hline IER3 & immediate early response 3 & $8870 \_$at & 8870 & 1,03 & 11,27718155 & $1,23 \mathrm{E}-04$ & $0,71 \%$ \\
\hline SERPINE2 & $\begin{array}{l}\text { serpin peptidase inhibitor, } \\
\text { clade } E \text { (nexin, plasminogen } \\
\text { activator inhibitor type } 1 \text { ), } \\
\text { member } 2\end{array}$ & $5270 \_$at & 5270 & 1,06 & 6,341013515 & $4,05 E-03$ & $4,88 \%$ \\
\hline LOC10028863 & hypothetical LOC100288637 & 100288637 & atl 00288637 & 1,06 & 6,249132666 & $1,18 \mathrm{E}-03$ & $2,55 \%$ \\
\hline GSTP1 & $\begin{array}{l}\text { glutathione S-transferase pi } \\
1\end{array}$ & 2950 at & 2950 & 1,06 & 7,120146409 & $7,18 \mathrm{E}-04$ & $1,95 \%$ \\
\hline ACSL4 & $\begin{array}{l}\text { acyl-CoA synthetase } \\
\text { long-chain family member } 4\end{array}$ & 2182 at & 2182 & 1,07 & 6,12492246 & $5,34 \mathrm{E}-05$ & $0,44 \%$ \\
\hline ZMIZ1 & $\begin{array}{l}\text { zinc finger, MIZ-type } \\
\text { containing } 1\end{array}$ & 57178_at & 57178 & 1,08 & 8,953868014 & $2,45 \mathrm{E}-04$ & $1,06 \%$ \\
\hline SLC10A5 & $\begin{array}{l}\text { solute carrier family } 10 \\
\text { (sodium/bile acid } \\
\text { cotransporter family), } \\
\text { member } 5\end{array}$ & 347051_at & 347051 & 1,08 & 6,224612778 & $9,23 \mathrm{E}-06$ & $0,16 \%$ \\
\hline CREB3L1 & $\begin{array}{l}\text { cAMP responsive element } \\
\text { binding protein } 3 \text {-like } 1\end{array}$ & 90993_at & 90993 & 1,08 & 7,108265808 & $2,69 E-06$ & $0,10 \%$ \\
\hline SYT11 & synaptotagmin XI & 23208 at & 23208 & 1,09 & 5,341932141 & $3,37 \mathrm{E}-04$ & $1,27 \%$ \\
\hline PCDHB13 & protocadherin beta 13 & 56123_at & 56123 & 1,09 & 6,382831539 & $3,27 \mathrm{E}-04$ & $1,25 \%$ \\
\hline TMBIM1 & $\begin{array}{l}\text { transmembrane BAX } \\
\text { inhibitor motif containing } 1\end{array}$ & 64114_at & 64114 & 1,09 & 6,899232446 & $1,73 E-03$ & $3,04 \%$ \\
\hline TRIM21 & tripartite motif containing 21 & 6737 at & 6737 & 1,10 & 8,320874252 & $1,26 \mathrm{E}-05$ & $0,19 \%$ \\
\hline PCDHB14 & protocadherin beta 14 & 56122 at & 56122 & 1,10 & 7,605161509 & $5,13 \mathrm{E}-05$ & $0,43 \%$ \\
\hline
\end{tabular}




\begin{tabular}{|c|c|c|c|c|c|c|c|}
\hline Symbol & Description & Probe & Gene & $\begin{array}{l}\text { logFC } \\
\text { Treatment- } \\
\text { Control }\end{array}$ & $\begin{array}{l}\text { AveExpr } \\
\text { Treatment- } \\
\text { Control }\end{array}$ & $\begin{array}{l}\text { P.Value } \\
\text { Treatment- } \\
\text { Control }\end{array}$ & $\begin{array}{l}\text { FDR } \\
\text { Treatment- } \\
\text { Control }\end{array}$ \\
\hline RBMS2 & $\begin{array}{l}\text { RNA binding motif, single } \\
\text { stranded interacting protein } \\
2\end{array}$ & 5939_at & 5939 & 1,10 & 7,532805283 & $2,22 \mathrm{E}-05$ & $0,27 \%$ \\
\hline CSF1 & $\begin{array}{l}\text { colony stimulating factor } 1 \\
\text { (macrophage) }\end{array}$ & 1435 at & 1435 & 1,10 & 7,232557251 & $6,01 \mathrm{E}-05$ & $0,47 \%$ \\
\hline HLA-C & $\begin{array}{l}\text { major histocompatibility } \\
\text { complex, class I, C }\end{array}$ & 3107_at & 3107 & 1,11 & 9,400715757 & $2,78 \mathrm{E}-05$ & $0,31 \%$ \\
\hline NFIA & nuclear factor $\mathrm{I} / \mathrm{A}$ & 4774_at & 4774 & 1,11 & 8,244579714 & $4,49 \mathrm{E}-04$ & $1,46 \%$ \\
\hline MYO10 & myosin X & 4651_at & 4651 & 1,12 & 7,362417639 & $8,09 \mathrm{E}-06$ & $0,15 \%$ \\
\hline MAP3K8 & $\begin{array}{l}\text { mitogen-activated protein } \\
\text { kinase kinase kinase } 8\end{array}$ & 1326 at & 1326 & 1,12 & 6,921510496 & $2,40 \mathrm{E}-05$ & $0,28 \%$ \\
\hline ZFP36L1 & $\begin{array}{l}\text { zinc finger protein } 36, \mathrm{C} 3 \mathrm{H} \\
\text { type-like } 1\end{array}$ & $677_{\text {_at }}$ & 677 & 1,13 & 10,11393638 & $1,23 \mathrm{E}-04$ & $0,71 \%$ \\
\hline PCDHB4 & protocadherin beta 4 & 56131 at & 56131 & 1,13 & 5,279374502 & $2,38 \mathrm{E}-04$ & $1,04 \%$ \\
\hline IGF2BP3 & $\begin{array}{l}\text { insulin-like growth factor } 2 \\
\text { mRNA binding protein } 3\end{array}$ & 10643 at & 10643 & 1,13 & 5,989384071 & $3,53 \mathrm{E}-03$ & $4,50 \%$ \\
\hline KRT15 & keratin 15 & 3866_at & 3866 & 1,14 & 6,439305018 & $3,30 \mathrm{E}-04$ & $1,25 \%$ \\
\hline PRSS12 & $\begin{array}{l}\text { protease, serine, } 12 \\
\text { (neurotrypsin, motopsin) }\end{array}$ & 8492 at & 8492 & 1,15 & 6,950500375 & $1,01 \mathrm{E}-03$ & $2,32 \%$ \\
\hline SEC24D & $\begin{array}{l}\text { SEC24 family, member D (S. } \\
\text { cerevisiae) }\end{array}$ & 9871 at & 9871 & 1,15 & 8,22444607 & $2,73 \mathrm{E}-05$ & $0,30 \%$ \\
\hline MICAL2 & $\begin{array}{l}\text { microtubule associated } \\
\text { monoxygenase, calponin and } \\
\text { LIM domain containing } 2\end{array}$ & 9645 at & 9645 & 1,15 & 6,141711945 & $3,24 \mathrm{E}-05$ & $0,33 \%$ \\
\hline BTN3A1 & $\begin{array}{l}\text { butyrophilin, subfamily } 3 \text {, } \\
\text { member A1 }\end{array}$ & 11119 at & 11119 & 1,16 & 5,190296349 & $1,22 \mathrm{E}-05$ & $0,19 \%$ \\
\hline RERG & $\begin{array}{l}\text { RAS-like, } \\
\text { estrogen-regulated, growth } \\
\text { in hibitor }\end{array}$ & 85004 at & 85004 & 1,17 & 9,365657718 & $2,17 \mathrm{E}-03$ & $3,46 \%$ \\
\hline RECK & $\begin{array}{l}\text { reversion-inducing-cysteine- } \\
\text { rich protein with kazal } \\
\text { motifs }\end{array}$ & 8434 at & 8434 & 1,18 & 6,895746662 & $1,19 \mathrm{E}-03$ & $2,55 \%$ \\
\hline CPNE4 & copine IV & 131034_at & 131034 & 1,18 & 7,21890412 & $8,60 \mathrm{E}-06$ & $0,15 \%$ \\
\hline PKIB & $\begin{array}{l}\text { protein kinase } \\
\text { (cAMP-dependent, } \\
\text { catalytic) inhibitor beta }\end{array}$ & 5570_at & 5570 & 1,19 & 7,804843382 & $1,38 \mathrm{E}-03$ & $2,75 \%$ \\
\hline MUC1 & $\begin{array}{l}\text { mucin } 1 \text {, cell surface } \\
\text { associated }\end{array}$ & 4582 at & 4582 & 1,19 & 10,08713053 & $4,41 \mathrm{E}-04$ & $1,45 \%$ \\
\hline C14orf132 & $\begin{array}{l}\text { chromosome } 14 \text { open } \\
\text { reading frame } 132\end{array}$ & 56967_at & 56967 & 1,20 & 7,746849978 & $1,23 \mathrm{E}-03$ & $2,59 \%$ \\
\hline SYTL5 & synaptotagmin-like 5 & 94122 at & 94122 & 1,20 & 8,141087654 & $2,00 \mathrm{E}-03$ & $3,29 \%$ \\
\hline EDN1 & end othelin 1 & 1906_at & 1906 & 1,21 & 6,815974591 & $4,25 \mathrm{E}-06$ & $0,11 \%$ \\
\hline LRP1 & $\begin{array}{l}\text { low density lipoprotein } \\
\text { receptor-related protein } 1\end{array}$ & 4035 at & 4035 & 1,22 & 7,428282893 & $4,94 \mathrm{E}-06$ & $0,12 \%$ \\
\hline GBP3 & guanylate binding protein 3 & 2635 at & 2635 & 1,22 & 5,602847219 & $3,75 \mathrm{E}-06$ & $0,11 \%$ \\
\hline FAM46C & $\begin{array}{l}\text { family with sequence } \\
\text { similarity } 46, \text { member } C\end{array}$ & 54855 at & 54855 & 1,22 & 7,666389632 & $4,60 \mathrm{E}-05$ & $0,40 \%$ \\
\hline SLC4A8 & $\begin{array}{l}\text { solute carrier family } 4, \\
\text { sodium bicarbonate } \\
\text { cotransporter, member } 8\end{array}$ & 9498 at & 9498 & 1,22 & 7,508882342 & $1,20 \mathrm{E}-04$ & $0,70 \%$ \\
\hline TNS1 & tensin 1 & 7145 at & 7145 & 1,22 & 8,050285528 & $8,54 \mathrm{E}-06$ & $0,15 \%$ \\
\hline $\mathrm{ABCD} 3$ & $\begin{array}{l}\text { ATP-binding cassette, } \\
\text { sub-family D (ALD), } \\
\text { member } 3\end{array}$ & 5825_at & 5825 & 1,23 & 8,335110877 & $3,81 \mathrm{E}-06$ & $0,11 \%$ \\
\hline DKK3 & $\begin{array}{l}\text { dickkopf homolog } 3 \\
\text { (Xenopus laevis) }\end{array}$ & 27122 at & 27122 & 1,24 & 7,431843058 & $9,10 \mathrm{E}-04$ & $2,18 \%$ \\
\hline FASN & fatty acid synthase & 2194_at & 2194 & 1,27 & 10,69221411 & $2,50 \mathrm{E}-04$ & $1,07 \%$ \\
\hline FRK & fyn-related kinase & 2444_at & 2444 & 1,28 & 7,295885411 & $4,19 \mathrm{E}-04$ & $1,40 \%$ \\
\hline SEPT9 & septin 9 & 10801 at & 10801 & 1,28 & 8,86983729 & $6,12 \mathrm{E}-05$ & $0,47 \%$ \\
\hline COL3A1 & collagen, type III, alpha 1 & 1281_at & 1281 & 1,30 & 7,573377467 & $4,91 \mathrm{E}-04$ & $1,55 \%$ \\
\hline RUNX2 & $\begin{array}{l}\text { runt-related transcription } \\
\text { factor } 2\end{array}$ & $860 \_$at & 860 & 1,30 & 7,431456704 & $2,23 \mathrm{E}-05$ & $0,27 \%$ \\
\hline
\end{tabular}




\begin{tabular}{|c|c|c|c|c|c|c|c|}
\hline Symbol & Description & Probe & Gene & $\begin{array}{l}\text { logFC } \\
\text { Treatment- } \\
\text { Control }\end{array}$ & $\begin{array}{l}\text { AveExpr } \\
\text { Treatment- } \\
\text { Control }\end{array}$ & $\begin{array}{l}\text { P.Value } \\
\text { Treatment- } \\
\text { Control }\end{array}$ & $\begin{array}{l}\text { FDR } \\
\text { Treatment- } \\
\text { Control }\end{array}$ \\
\hline B2M & beta-2-microglobulin & 567_at & 567 & 1,30 & 9,556534906 & $9,60 \mathrm{E}-07$ & $0,07 \%$ \\
\hline LGALS3BP & $\begin{array}{l}\text { lectin, galactoside-binding, } \\
\text { soluble, } 3 \text { binding protein }\end{array}$ & 3959 at & 3959 & 1,31 & 9,949297705 & $3,19 \mathrm{E}-05$ & $0,33 \%$ \\
\hline PDE4D & $\begin{array}{l}\text { phosphodiesterase 4D, } \\
\text { cAMP-specific }\end{array}$ & 5144_at & 5144 & 1,31 & 6,718386857 & $2,04 \mathrm{E}-06$ & $0,09 \%$ \\
\hline NEURL & $\begin{array}{l}\text { neuralized homolog } \\
\text { (Drosophila) }\end{array}$ & 9148 at & 9148 & 1,31 & 8,241856985 & $2,07 E-04$ & $0,96 \%$ \\
\hline SEMA3B & $\begin{array}{l}\text { sema domain, } \\
\text { immunoglobulin domain (lg), } \\
\text { short basic domain, secreted, } \\
\text { (semaphorin) 3B }\end{array}$ & 7869 at & 7869 & 1,31 & 6,255981287 & $5,30 \mathrm{E}-06$ & $0,12 \%$ \\
\hline STAT1 & $\begin{array}{l}\text { signal transducer and } \\
\text { activator of transcription } 1 \text {, } \\
91 \mathrm{kDa}\end{array}$ & 6772 at & 6772 & 1,31 & 10,86613724 & $2,01 \mathrm{E}-05$ & $0,25 \%$ \\
\hline SLC9A7 & $\begin{array}{l}\text { solute carrier family } 9 \\
\text { (sodium/hydrogen } \\
\text { exchanger), member } 7\end{array}$ & $84679_{\text {_at }}$ & 84679 & 1,31 & 7,883368851 & $2,28 \mathrm{E}-04$ & $1,02 \%$ \\
\hline CELSR2 & $\begin{array}{l}\text { cadherin, EGF LAG } \\
\text { seven-pass G-type receptor } 2 \\
\text { (flamingo homolog, } \\
\text { Drosophila) }\end{array}$ & 1952_at & 1952 & 1,33 & 9,838710785 & $2,33 \mathrm{E}-04$ & $1,04 \%$ \\
\hline IFITM3 & $\begin{array}{l}\text { interferon induced } \\
\text { transmembrane protein } 3 \\
(1-8 U)\end{array}$ & $10410_{\text {at }}$ & 10410 & 1,33 & 12,44956283 & $6,47 \mathrm{E}-06$ & $0,13 \%$ \\
\hline SPRED1 & $\begin{array}{l}\text { sprouty-related, EVH1 } \\
\text { domain containing } 1\end{array}$ & 161742 at & 161742 & 1,34 & 7,346541722 & $2,79 \mathrm{E}-05$ & $0,31 \%$ \\
\hline RPS4Y1 & $\begin{array}{l}\text { ribosomal protein S4, } \\
\text { Y-linked } 1\end{array}$ & 6192 at & 6192 & 1,35 & 5,552372194 & $2,44 \mathrm{E}-04$ & $1,06 \%$ \\
\hline C5orf 4 & $\begin{array}{l}\text { chromosome } 5 \text { open reading } \\
\text { frame } 4\end{array}$ & 10826_at & 10826 & 1,37 & 6,702335325 & $4,59 \mathrm{E}-06$ & $0,12 \%$ \\
\hline UBE2L6 & $\begin{array}{l}\text { ubiquitin-conjugating } \\
\text { enzyme E2L } 6\end{array}$ & 9246 at & 9246 & 1,37 & 9,119108197 & $9,02 \mathrm{E}-06$ & $0,16 \%$ \\
\hline MGP & matrix Gla protein & 4256_at & 4256 & 1,38 & 9,541935291 & $6,31 \mathrm{E}-04$ & $1,81 \%$ \\
\hline C19orf66 & $\begin{array}{l}\text { chromosome } 19 \text { open } \\
\text { reading frame } 66\end{array}$ & 55337 at & 55337 & 1,38 & 8,086477939 & $3,30 \mathrm{E}-06$ & $0,10 \%$ \\
\hline GMPR & $\begin{array}{l}\text { guanosine monophosphate } \\
\text { reductase }\end{array}$ & 2766 at & 2766 & 1,39 & 7,875015767 & $3,16 \mathrm{E}-05$ & $0,33 \%$ \\
\hline ARHGAP29 & $\begin{array}{l}\text { Rho GTPase activating } \\
\text { protein } 29\end{array}$ & 9411 at & 9411 & 1,39 & 7,421044507 & $6,63 \mathrm{E}-04$ & $1,86 \%$ \\
\hline$M \times 2$ & $\begin{array}{l}\text { myxovirus (influenza virus) } \\
\text { resistance } 2 \text { (mouse) }\end{array}$ & 4600 at & 4600 & 1,39 & 6,438006749 & $3,06 \mathrm{E}-04$ & $1,20 \%$ \\
\hline FAM38B & $\begin{array}{l}\text { family with sequence } \\
\text { similarity } 38 \text {, member B }\end{array}$ & 63895 at & 63895 & 1,39 & 5,928980827 & $2,15 \mathrm{E}-04$ & $0,98 \%$ \\
\hline PARP12 & $\begin{array}{l}\text { poly (ADP-ribose) } \\
\text { polymerase family, member } \\
12\end{array}$ & 64761 at & 64761 & 1,40 & 9,611994527 & $4,31 \mathrm{E}-05$ & $0,39 \%$ \\
\hline TMC5 & $\begin{array}{l}\text { transmembrane channel-like } \\
5\end{array}$ & 79838 at & 79838 & 1,40 & 7,313439219 & $3,79 \mathrm{E}-07$ & $0,04 \%$ \\
\hline DTX3L & deltex 3-like (Drosophila) & 151636_at & 151636 & 1,40 & 9,61943333 & $2,44 \mathrm{E}-05$ & $0,28 \%$ \\
\hline TAP1 & $\begin{array}{l}\text { transporter } 1 \text {, ATP-binding } \\
\text { cassette, sub-family B } \\
\text { (MDR/TAP) }\end{array}$ & 6890 at & 6890 & 1,41 & 9,007700765 & $7,38 \mathrm{E}-07$ & $0,06 \%$ \\
\hline TPM2 & tropomyosin 2 (beta) & 7169 at & 7169 & 1,41 & 6,315382133 & 3,17E-05 & $0,33 \%$ \\
\hline $\mathrm{HOXC13}$ & homeobox $\mathrm{C} 13$ & 3229_at & 3229 & 1,41 & 8,643856601 & $4,07 \mathrm{E}-05$ & $0,38 \%$ \\
\hline IFITM1 & $\begin{array}{l}\text { interferon induced } \\
\text { transmembrane protein } 1 \\
(9-27)\end{array}$ & 8519 at & 8519 & 1,41 & 11,72118703 & $2,46 \mathrm{E}-05$ & $0,28 \%$ \\
\hline MYO18A & myosin XVIIIA & 399687 at & 399687 & 1,42 & 7,825208346 & $1,18 \mathrm{E}-06$ & $0,07 \%$ \\
\hline PDGFRA & $\begin{array}{l}\text { platelet-derived growth } \\
\text { factor receptor, alpha } \\
\text { polypeptide }\end{array}$ & 5156_at & 5156 & 1,44 & 5,90701297 & $1,19 \mathrm{E}-03$ & $2,55 \%$ \\
\hline
\end{tabular}




\begin{tabular}{|c|c|c|c|c|c|c|c|}
\hline Symbol & Description & Probe & Gene & $\begin{array}{l}\log \mathrm{FC} \\
\text { Treatment- } \\
\text { Control }\end{array}$ & $\begin{array}{l}\text { AveExpr } \\
\text { Treatment- } \\
\text { Control }\end{array}$ & $\begin{array}{l}\text { P.Value } \\
\text { Treatment- } \\
\text { Control }\end{array}$ & $\begin{array}{l}\text { FDR } \\
\text { Treatment- } \\
\text { Control }\end{array}$ \\
\hline $\mathrm{MSI} 2$ & $\begin{array}{l}\text { musashi homolog } 2 \\
\text { (Drosophila) }\end{array}$ & 124540 at & 124540 & 1,44 & 8,991082904 & $1,08 \mathrm{E}-06$ & $0,07 \%$ \\
\hline TRIM14 & tripartite motif containing 14 & 9830_at & 9830 & 1,45 & 8,627528615 & $5,59 \mathrm{E}-06$ & $0,13 \%$ \\
\hline COL6A3 & collagen, type VI, alpha 3 & 1293 at & 1293 & 1,45 & 6,62163837 & $6,57 \mathrm{E}-04$ & $1,85 \%$ \\
\hline ETV 5 & ets variant 5 & 2119 at & 2119 & 1,45 & 5,559421664 & $7,28 \mathrm{E}-06$ & $0,14 \%$ \\
\hline TMPRSS4 & $\begin{array}{l}\text { transmembrane protease, } \\
\text { serine } 4\end{array}$ & 56649 at & 56649 & 1,46 & 6,98786033 & $1,14 \mathrm{E}-04$ & $0,68 \%$ \\
\hline ARHGEF6 & $\begin{array}{l}\text { Rac/Cdc42 guanine } \\
\text { nucleotide exchange factor } \\
\text { (GEF) } 6\end{array}$ & 9459_at & 9459 & 1,46 & 7,140665186 & $5,97 \mathrm{E}-06$ & $0,13 \%$ \\
\hline C6orf192 & $\begin{array}{l}\text { chromosome } 6 \text { open reading } \\
\text { frame } 192\end{array}$ & 116843_at & 116843 & 1,46 & 6,517304181 & $2,64 \mathrm{E}-05$ & $0,30 \%$ \\
\hline SAMHD1 & $\begin{array}{l}\text { SAM domain and HD } \\
\text { domain } 1\end{array}$ & 25939 at & 25939 & 1,47 & 8,866562844 & $3,51 \mathrm{E}-06$ & $0,10 \%$ \\
\hline LOH3CR2A & $\begin{array}{l}\text { loss of heterozygosity, } 3, \\
\text { chromosomal region } 2 \text {, gene } \\
\text { A }\end{array}$ & $29931_{\text {_at }}$ & 29931 & 1,47 & 6,54651044 & $8,13 E-05$ & $0,55 \%$ \\
\hline BGN & biglycan & 633 at & 633 & 1,49 & 7,775465713 & $5,12 \mathrm{E}-05$ & $0,43 \%$ \\
\hline GOLM1 & golgi membrane protein 1 & 51280 at & 51280 & 1,49 & 8,826160737 & $3,64 \mathrm{E}-07$ & $0,04 \%$ \\
\hline BNC2 & basonuclin 2 & 54796_at & 54796 & 1,51 & 5,806704432 & $2,95 \mathrm{E}-04$ & $1,19 \%$ \\
\hline CYP1B1 & $\begin{array}{l}\text { cytochrome } \mathrm{P} 450 \text {, family } 1 \text {, } \\
\text { subfamily } \mathrm{B} \text {, polypeptide } 1\end{array}$ & 1545 at & 1545 & 1,53 & 8,681359645 & $3,30 \mathrm{E}-06$ & $0,10 \%$ \\
\hline C10orf81 & $\begin{array}{l}\text { chromosome } 10 \text { open } \\
\text { reading frame } 81\end{array}$ & 79949 at & 79949 & 1,53 & 7,898296449 & $3,23 E-08$ & $0,02 \%$ \\
\hline MUC5B & $\begin{array}{l}\text { mucin } 5 \mathrm{~B} \text {, oligomeric } \\
\text { mucus/gel-forming }\end{array}$ & 727897 at & 727897 & 1,54 & 8,626094708 & $3,92 \mathrm{E}-05$ & $0,37 \%$ \\
\hline SLC7A5 & $\begin{array}{l}\text { solute carrier family } 7 \\
\text { (cationic amino acid } \\
\text { transporter, y+ system), } \\
\text { member } 5\end{array}$ & 8140 at & 8140 & 1,54 & 11,50081234 & 1,13E-05 & $0,18 \%$ \\
\hline MSN & moesin & 4478 at & 4478 & 1,54 & 6,403989318 & $7,51 \mathrm{E}-04$ & $2,00 \%$ \\
\hline FRMD3 & FERM domain containing 3 & 257019 at & 257019 & 1,54 & 7,540879962 & $1,48 \mathrm{E}-06$ & $0,08 \%$ \\
\hline SPRY 4 & $\begin{array}{l}\text { sprouty homolog } 4 \\
\text { (Drosophila) }\end{array}$ & 81848 at & 81848 & 1,54 & 6,33955723 & $4,57 \mathrm{E}-05$ & $0,40 \%$ \\
\hline BST2 & $\begin{array}{l}\text { bone marrow stromal cell } \\
\text { antigen } 2\end{array}$ & 684 at & 684 & 1,54 & 8,157384428 & $2,49 \mathrm{E}-03$ & $3,73 \%$ \\
\hline NMI & $\begin{array}{l}\mathrm{N} \text {-myc (and STAT) } \\
\text { interactor }\end{array}$ & 9111 at & 9111 & 1,56 & 6,479639869 & $1,03 E-05$ & $0,17 \%$ \\
\hline GSTM1 & $\begin{array}{l}\text { glutathione S-transferase mu } \\
1\end{array}$ & 2944 at & 2944 & 1,56 & 6,009259918 & $4,05 \mathrm{E}-04$ & $1,38 \%$ \\
\hline MIR21 & microRNA 21 & 406991 at & 406991 & 1,56 & 9,592869762 & $1,54 \mathrm{E}-04$ & $0,82 \%$ \\
\hline CRAT & carnitine $\mathrm{O}$-acetyltransferase & 1384 at & 1384 & 1,57 & 7,614550187 & $1,33 \mathrm{E}-07$ & $0,03 \%$ \\
\hline PEG10 & paternally expressed 10 & 23089 at & 23089 & 1,58 & 6,878569508 & $3,06 \mathrm{E}-07$ & $0,04 \%$ \\
\hline CCKAR & cholecystokinin A receptor & 886 at & 886 & 1,58 & 6,062904045 & $6,69 \mathrm{E}-04$ & $1,87 \%$ \\
\hline TFF1 & trefoil factor 1 & 7031 at & 7031 & 1,58 & 11,82069475 & $2,27 \mathrm{E}-05$ & $0,27 \%$ \\
\hline CNN3 & calponin 3 , acidic & 1266_at & 1266 & 1,59 & 5,533918747 & $2,55 \mathrm{E}-03$ & $3,79 \%$ \\
\hline HEG 1 & HEG homolog 1 (zebrafish) & 57493 at & 57493 & 1,59 & 6,262465196 & $8,46 \mathrm{E}-06$ & $0,15 \%$ \\
\hline MT1E & metallothionein $1 \mathrm{E}$ & 4493 at & 4493 & 1,60 & 7,826020391 & $2,32 \mathrm{E}-05$ & $0,28 \%$ \\
\hline RARRES3 & $\begin{array}{l}\text { retinoic acid receptor } \\
\text { responder (tazarotene } \\
\text { induced) } 3\end{array}$ & $5920 \_$at & 5920 & 1,60 & 7,050441904 & $8,21 \mathrm{E}-06$ & $0,15 \%$ \\
\hline LXN & latexin & 56925 at & 56925 & 1,61 & 7,197593556 & $1,25 \mathrm{E}-05$ & $0,19 \%$ \\
\hline CADPS2 & $\begin{array}{l}\text { Ca++-dependent secretion } \\
\text { activator } 2\end{array}$ & 93664_at & 93664 & 1,61 & 8,038317549 & $1,53 \mathrm{E}-05$ & $0,21 \%$ \\
\hline TAP2 & $\begin{array}{l}\text { transporter } 2 \text {, ATP-binding } \\
\text { cassette, sub-family B } \\
\text { (MDR/TAP) }\end{array}$ & 6891 at & 6891 & 1,62 & 7,759885972 & $4,56 \mathrm{E}-07$ & $0,04 \%$ \\
\hline USP18 & $\begin{array}{l}\text { ubiquitin specific peptidase } \\
18\end{array}$ & 11274_at & 11274 & 1,62 & 9,230878782 & $1,42 \mathrm{E}-05$ & $0,21 \%$ \\
\hline NRIP1 & $\begin{array}{l}\text { nuclear receptor interacting } \\
\text { protein } 1\end{array}$ & 8204 at & 8204 & 1,62 & 9,3695053 & $1,31 \mathrm{E}-05$ & $0,20 \%$ \\
\hline
\end{tabular}




\begin{tabular}{|c|c|c|c|c|c|c|c|}
\hline Symbol & Description & Probe & Gene & $\begin{array}{l}\log \mathrm{FC} \\
\text { Treatment- } \\
\text { Control }\end{array}$ & $\begin{array}{l}\text { AveExpr } \\
\text { Treatment- } \\
\text { Control }\end{array}$ & $\begin{array}{l}\text { P.Value } \\
\text { Treatment- } \\
\text { Control }\end{array}$ & $\begin{array}{l}\text { FDR } \\
\text { Treatment- } \\
\text { Control }\end{array}$ \\
\hline $\mathrm{ABCC} 3$ & $\begin{array}{l}\text { ATP-binding cassette, } \\
\text { sub-family C (CFTR/MRP), } \\
\text { member } 3\end{array}$ & 8714_at & 8714 & 1,63 & 7,961604802 & $2,22 \mathrm{E}-03$ & $3,49 \%$ \\
\hline IFIH1 & $\begin{array}{l}\text { interferon induced with } \\
\text { helicase } C \text { domain } 1\end{array}$ & 64135 at & 64135 & 1,63 & 8,092288478 & 1,74E-05 & $0,23 \%$ \\
\hline DDR2 & $\begin{array}{l}\text { discoidin domain receptor } \\
\text { tyrosine kinase } 2\end{array}$ & 4921_at & 4921 & 1,64 & 6,752625408 & $9,34 \mathrm{E}-04$ & $2,22 \%$ \\
\hline IFI35 & interferon-induced protein 35 & 3430_at & 3430 & 1,64 & 9,125504844 & 1,23E-05 & $0,19 \%$ \\
\hline TLR3 & toll-like receptor 3 & 7098 at & 7098 & 1,65 & 6,344474864 & $2,95 \mathrm{E}-06$ & $0,10 \%$ \\
\hline CD44 & $\begin{array}{l}\text { CD44 molecule (Indian } \\
\text { blood group) }\end{array}$ & $960 \_$at & 960 & 1,65 & 8,94898628 & $1,71 \mathrm{E}-04$ & $0,86 \%$ \\
\hline UCA1 & $\begin{array}{l}\text { urothelial cancer associated } \\
1 \text { (non-protein coding) }\end{array}$ & 652995_at & 652995 & 1,65 & 5,628423175 & $8,56 \mathrm{E}-06$ & $0,15 \%$ \\
\hline PCDHB8 & protocadherin beta 8 & 56128 at & 56128 & 1,66 & 5,446032138 & $3,67 \mathrm{E}-04$ & $1,31 \%$ \\
\hline NT5E & 5'-nucleotidase, ecto (CD73) & 4907_at & 4907 & 1,66 & 5,832109506 & $4,23 \mathrm{E}-03$ & $4,98 \%$ \\
\hline SVEP1 & $\begin{array}{l}\text { sushi, von Willebrand factor } \\
\text { type A, EGF and pentraxin } \\
\text { domain containing } 1\end{array}$ & 79987_at & 79987 & 1,66 & 6,251339017 & $3,49 \mathrm{E}-04$ & $1,28 \%$ \\
\hline NCRNA00052 & non-protein coding RNA 52 & 145978_at & 145978 & 1,67 & 5,929461106 & $1,08 \mathrm{E}-05$ & $0,18 \%$ \\
\hline TIAM1 & $\begin{array}{l}\text { T-cell lymphoma invasion } \\
\text { and metastasis } 1\end{array}$ & 7074_at & 7074 & 1,68 & 8,773042075 & $2,49 \mathrm{E}-06$ & $0,10 \%$ \\
\hline HLA-B & $\begin{array}{l}\text { major histocompatibility } \\
\text { complex, class I, B }\end{array}$ & 3106 at & 3106 & 1,69 & 9,07722762 & $4,94 \mathrm{E}-05$ & $0,42 \%$ \\
\hline BDKRB2 & bradykinin receptor B2 & 624 at & 624 & 1,71 & 7,174015988 & $2,90 \mathrm{E}-06$ & $0,10 \%$ \\
\hline LDHB & lactate dehydrogenase $B$ & 3945_at & 3945 & 1,71 & 6,733305254 & $6,21 \mathrm{E}-04$ & $1,79 \%$ \\
\hline EGR1 & early growth response 1 & 1958_at & 1958 & 1,71 & 9,564396843 & $3,65 \mathrm{E}-03$ & $4,58 \%$ \\
\hline SYT12 & synaptotagmin XII & 91683 at & 91683 & 1,71 & 8,072182319 & $4,98 \mathrm{E}-05$ & $0,42 \%$ \\
\hline GPX8 & $\begin{array}{l}\text { glutathione peroxidase } 8 \\
\text { (putative) }\end{array}$ & 493869_at & 493869 & 1,73 & 5,984429246 & $1,28 \mathrm{E}-03$ & $2,63 \%$ \\
\hline FSTL1 & follistatin-like 1 & 11167 at & 11167 & 1,74 & 6,881321453 & $1,63 \mathrm{E}-04$ & $0,84 \%$ \\
\hline TGFB3 & $\begin{array}{l}\text { transforming growth factor, } \\
\text { beta } 3\end{array}$ & 7043_at & 7043 & 1,74 & 8,461791881 & $5,04 \mathrm{E}-06$ & $0,12 \%$ \\
\hline SP110 & SP110 nuclear body protein & 3431_at & 3431 & 1,76 & 7,796298243 & $1,42 \mathrm{E}-05$ & $0,21 \%$ \\
\hline NAV1 & neuron navigator 1 & 89796_at & 89796 & 1,76 & 7,05274052 & $7,19 \mathrm{E}-08$ & $0,02 \%$ \\
\hline ALDOC & $\begin{array}{l}\text { aldolase C, } \\
\text { fructose-bisphosphate }\end{array}$ & $230 \_$at & 230 & 1,76 & 8,483623162 & $1,17 \mathrm{E}-03$ & $2,53 \%$ \\
\hline RGS16 & $\begin{array}{l}\text { regulator of G-protein } \\
\text { signaling } 16\end{array}$ & 6004_at & 6004 & 1,76 & 8,417586262 & $5,31 \mathrm{E}-07$ & $0,05 \%$ \\
\hline $\mathrm{MX1}$ & $\begin{array}{l}\text { myxovirus (influenza virus) } \\
\text { resistance } 1 \text {, } \\
\text { interferon-inducible protein } \\
\text { p78 (mouse) }\end{array}$ & 4599_at & 4599 & 1,77 & 6,449030253 & $8,44 \mathrm{E}-05$ & $0,56 \%$ \\
\hline SPATS2L & $\begin{array}{l}\text { spermatogenesis associated, } \\
\text { serine-rich 2-like }\end{array}$ & $26010_{\text {at }}$ at & 26010 & 1,78 & 7,178799967 & $3,46 \mathrm{E}-04$ & $1,28 \%$ \\
\hline TSKU & $\begin{array}{l}\text { tsukushi small leucine rich } \\
\text { proteoglycan homolog } \\
\text { (Xenopus laevis) }\end{array}$ & 25987 at & 25987 & 1,78 & 10,32739865 & $2,64 \mathrm{E}-07$ & $0,04 \%$ \\
\hline USP 41 & $\begin{array}{l}\text { ubiquitin specific peptidase } \\
41\end{array}$ & 373856_at & 373856 & 1,79 & 8,310724967 & $3,01 \mathrm{E}-06$ & $0,10 \%$ \\
\hline DDX58 & $\begin{array}{l}\text { DEAD (Asp-Glu-Ala-Asp) } \\
\text { box polypeptide } 58\end{array}$ & 23586 at & 23586 & 1,81 & 9,447755245 & $1,66 \mathrm{E}-05$ & $0,22 \%$ \\
\hline LHFP & $\begin{array}{l}\text { lipoma HMGIC fusion } \\
\text { partner }\end{array}$ & 10186 at & 10186 & 1,82 & 8,218518602 & $1,34 \mathrm{E}-03$ & $2,72 \%$ \\
\hline PLSCR1 & phospholipid scramblase 1 & 5359_at & 5359 & 1,83 & 9,442454124 & $4,88 \mathrm{E}-06$ & $0,12 \%$ \\
\hline FBN1 & fibrillin 1 & 2200_at & 2200 & 1,83 & 6,081068838 & $3,26 \mathrm{E}-04$ & $1,25 \%$ \\
\hline MBOAT1 & $\begin{array}{l}\text { membrane bound } \\
\text { O-acyltransferase domain } \\
\text { containing } 1\end{array}$ & 154141_at & 154141 & 1,85 & 9,042516241 & $1,50 \mathrm{E}-06$ & $0,08 \%$ \\
\hline CYBRD1 & cytochrome b reductase 1 & 79901 at & 79901 & 1,86 & 7,480524932 & $8,74 \mathrm{E}-04$ & $2,13 \%$ \\
\hline CTSD & cathepsin D & 1509_at & 1509 & 1,86 & 10,95111405 & $4,22 \mathrm{E}-05$ & $0,39 \%$ \\
\hline SP100 & SP100 nuclear antigen & 6672 at & 6672 & 1,86 & 8,253230328 & 3,07E-05 & $0,33 \%$ \\
\hline NRXN3 & neurexin 3 & 9369_at & 9369 & 1,89 & 7,018792506 & $2,73 \mathrm{E}-07$ & $0,04 \%$ \\
\hline
\end{tabular}




\begin{tabular}{|c|c|c|c|c|c|c|c|}
\hline Symbol & Description & Probe & Gene & $\begin{array}{l}\log \mathrm{FC} \\
\text { Treatment- } \\
\text { Control }\end{array}$ & $\begin{array}{l}\text { AveExpr } \\
\text { Treatment- } \\
\text { Control }\end{array}$ & $\begin{array}{l}\text { P.Value } \\
\text { Treatment- } \\
\text { Control }\end{array}$ & $\begin{array}{l}\text { FDR } \\
\text { Treatment- } \\
\text { Control }\end{array}$ \\
\hline PLK2 & polo-like kinase 2 & 10769_at & 10769 & 1,89 & 10,80404779 & $5,49 \mathrm{E}-06$ & $0,13 \%$ \\
\hline ANPEP & $\begin{array}{l}\text { alanyl (membrane) } \\
\text { aminopeptidase }\end{array}$ & $290 \_$at & 290 & 1,90 & 6,488611603 & $2,47 \mathrm{E}-06$ & $0,10 \%$ \\
\hline GBP1 & $\begin{array}{l}\text { guanylate binding protein } 1 \text {, } \\
\text { interferon-inducible }\end{array}$ & 2633_at & 2633 & 1,90 & 5,20424888 & $8,55 \mathrm{E}-04$ & $2,12 \%$ \\
\hline EHF & ets homologous factor & 26298 at & 26298 & 1,91 & 9,02228668 & $1,76 \mathrm{E}-06$ & $0,09 \%$ \\
\hline IFIT5 & $\begin{array}{l}\text { interferon-induced protein } \\
\text { with tetratricopeptide } \\
\text { repeats } 5\end{array}$ & 24138 at & 24138 & 1,91 & 8,682144239 & $9,93 \mathrm{E}-06$ & $0,17 \%$ \\
\hline SAMD9L & $\begin{array}{l}\text { sterile alpha motif domain } \\
\text { containing 9-like }\end{array}$ & 219285_at & 219285 & 1,92 & 6,009456877 & $6,70 \mathrm{E}-05$ & $0,50 \%$ \\
\hline PSMB9 & $\begin{array}{l}\text { proteasome (prosome, } \\
\text { macropain) subunit, beta } \\
\text { type, } 9 \text { (large } \\
\text { multifunctional peptidase 2) }\end{array}$ & 5698_at & 5698 & 1,93 & 7,776233841 & $4,02 \mathrm{E}-07$ & $0,04 \%$ \\
\hline SUSD3 & sushi domain containing 3 & 203328 at & 203328 & 1,93 & 7,909122887 & $3,00 \mathrm{E}-05$ & $0,32 \%$ \\
\hline $\mathrm{H} 19$ & $\begin{array}{l}\text { H19, imprinted maternally } \\
\text { expressed transcript } \\
\text { (non-protein coding) }\end{array}$ & 283120 at & 283120 & 1,95 & 9,255262101 & $3,01 E-06$ & $0,10 \%$ \\
\hline MPPED2 & $\begin{array}{l}\text { metallophosphoesterase } \\
\text { domain containing } 2\end{array}$ & 744 at & 744 & 1,95 & 8,348080119 & $2,83 \mathrm{E}-04$ & $1,16 \%$ \\
\hline IFI6 & $\begin{array}{l}\text { interferon, alpha-inducible } \\
\text { protein } 6\end{array}$ & 2537 at & 2537 & 1,96 & 11,51967212 & $7,92 \mathrm{E}-05$ & $0,54 \%$ \\
\hline SLC7A11 & $\begin{array}{l}\text { solute carrier family } 7, \\
\text { (cationic amino acid } \\
\text { transporter, y+ system) } \\
\text { member } 11\end{array}$ & 23657 at & 23657 & 2,01 & 7,294256647 & $7,65 \mathrm{E}-05$ & $0,53 \%$ \\
\hline EMP3 & $\begin{array}{l}\text { epithelial membrane protein } \\
3\end{array}$ & 2014_at & 2014 & 2,09 & 7,371324685 & $4,76 \mathrm{E}-06$ & $0,12 \%$ \\
\hline APOL6 & apolipoprotein L, 6 & $80830 \_$at & 80830 & 2,11 & 6,276044523 & $8,09 \mathrm{E}-07$ & $0,06 \%$ \\
\hline DUSP4 & $\begin{array}{l}\text { dual specificity phosphatase } \\
4\end{array}$ & 1846 at & 1846 & 2,12 & 6,673716905 & $2,51 \mathrm{E}-06$ & $0,10 \%$ \\
\hline IFIT2 & $\begin{array}{l}\text { interferon-induced protein } \\
\text { with tetratricopeptide } \\
\text { repeats } 2\end{array}$ & 3433_at & 3433 & 2,14 & 5,99411937 & $2,22 \mathrm{E}-06$ & $0,09 \%$ \\
\hline SDK2 & sidekick homolog 2 (chicken) & 54549_at & 54549 & 2,16 & 6,856323797 & $9,70 \mathrm{E}-06$ & $0,16 \%$ \\
\hline AFF3 & $\begin{array}{l}\text { AF4/FMR2 family, member } \\
3\end{array}$ & 3899 at & 3899 & 2,16 & 8,053518591 & $6,80 \mathrm{E}-07$ & $0,06 \%$ \\
\hline EGR3 & early growth response 3 & 1960_at & 1960 & 2,16 & 7,449399602 & $6,57 \mathrm{E}-06$ & $0,13 \%$ \\
\hline CMPK2 & $\begin{array}{l}\text { cytidine monophosphate } \\
\text { (UMP-CMP) kinase 2, } \\
\text { mitochondrial }\end{array}$ & 129607_at & 129607 & 2,21 & 8,542190577 & $5,68 \mathrm{E}-06$ & $0,13 \%$ \\
\hline TGFBI & $\begin{array}{l}\text { transforming growth factor, } \\
\text { beta-induced, } 68 \mathrm{kDa}\end{array}$ & 7045 at & 7045 & 2,21 & 6,406009873 & $5,39 \mathrm{E}-04$ & $1,65 \%$ \\
\hline PARP14 & $\begin{array}{l}\text { poly (ADP-ribose) } \\
\text { polymerase family, member } \\
14\end{array}$ & 54625 at & 54625 & 2,22 & 9,027314074 & $7,14 \mathrm{E}-06$ & $0,14 \%$ \\
\hline CAV1 & $\begin{array}{l}\text { caveolin } 1 \text {, caveolae protein, } \\
22 \mathrm{kDa}\end{array}$ & 857 at & 857 & 2,23 & 6,684728209 & $3,76 \mathrm{E}-05$ & $0,36 \%$ \\
\hline MT2A & metallothionein $2 \mathrm{~A}$ & 4502 at & 4502 & 2,23 & 11,38264217 & $3,99 \mathrm{E}-07$ & $0,04 \%$ \\
\hline BDKRB1 & bradykinin receptor $\mathrm{B} 1$ & 623 at & 623 & 2,26 & 7,595901607 & $4,00 \mathrm{E}-06$ & $0,11 \%$ \\
\hline FAM5B & $\begin{array}{l}\text { family with sequence } \\
\text { similarity } 5, \text { member B }\end{array}$ & 57795_at & 57795 & 2,33 & 7,990252174 & $2,16 \mathrm{E}-07$ & $0,04 \%$ \\
\hline TGM2 & $\begin{array}{l}\text { transglutaminase } 2 \text { (C } \\
\text { polypeptide, } \\
\text { protein-glutamine-gamma- } \\
\text { glutamyltransferase) }\end{array}$ & 7052 at & 7052 & 2,35 & 7,78116388 & $2,88 \mathrm{E}-05$ & $0,31 \%$ \\
\hline LPAR1 & $\begin{array}{l}\text { lysophosphatidic acid } \\
\text { receptor } 1\end{array}$ & 1902 at & 1902 & 2,37 & 6,575360477 & $2,56 \mathrm{E}-05$ & $0,29 \%$ \\
\hline HERC6 & hect domain and RLD 6 & 55008 at & 55008 & 2,39 & 8,716003657 & $1,64 \mathrm{E}-04$ & $0,84 \%$ \\
\hline AGR2 & $\begin{array}{l}\text { anterior gradient homolog } 2 \\
\text { (Xenopus laevis) }\end{array}$ & 10551 at & 10551 & 2,40 & 7,146446502 & $2,06 \mathrm{E}-08$ & $0,02 \%$ \\
\hline
\end{tabular}




\begin{tabular}{|c|c|c|c|c|c|c|c|}
\hline Symbol & Description & Probe & Gene & $\begin{array}{l}\text { logFC } \\
\text { Treatment- } \\
\text { Control }\end{array}$ & $\begin{array}{l}\text { AveExpr } \\
\text { Treatment- } \\
\text { Control }\end{array}$ & $\begin{array}{l}\text { P.Value } \\
\text { Treatment- } \\
\text { Control }\end{array}$ & $\begin{array}{l}\text { FDR } \\
\text { Treatment- } \\
\text { Control }\end{array}$ \\
\hline IFI16 & $\begin{array}{l}\text { interferon, gamma-inducible } \\
\text { protein } 16\end{array}$ & 3428 at & 3428 & 2,45 & 5,317007642 & $6,65 \mathrm{E}-04$ & $1,86 \%$ \\
\hline TCF21 & transcription factor 21 & 6943_at & 6943 & 2,47 & 6,808045795 & $5,20 \mathrm{E}-06$ & $0,12 \%$ \\
\hline MUCL1 & mucin-like 1 & 118430_at & 118430 & 2,48 & 8,156771675 & $2,39 \mathrm{E}-08$ & $0,02 \%$ \\
\hline LOX & lysyl oxidase & 4015 at & 4015 & 2,50 & 6,523605374 & $4,87 \mathrm{E}-05$ & $0,42 \%$ \\
\hline PADI2 & $\begin{array}{l}\text { peptidyl arginine deiminase, } \\
\text { type II }\end{array}$ & 11240 at & 11240 & 2,51 & 7,612124221 & $7,07 \mathrm{E}-08$ & $0,02 \%$ \\
\hline PARP9 & $\begin{array}{l}\text { poly (ADP-ribose) } \\
\text { polymerase family, member } 9\end{array}$ & 83666 at & 83666 & 2,52 & 8,043390009 & $1,92 \mathrm{E}-05$ & $0,24 \%$ \\
\hline VIM & vimentin & 7431 at & 7431 & 2,57 & 7,657125406 & 1,13E-05 & $0,18 \%$ \\
\hline CTGF & $\begin{array}{l}\text { connective tissue growth } \\
\text { factor }\end{array}$ & 1490_at & 1490 & 2,65 & 8,288413238 & 1,51E-05 & $0,21 \%$ \\
\hline XAF1 & XIAP associated factor 1 & 54739 at & 54739 & 2,66 & 10,23470051 & $1,57 \mathrm{E}-04$ & $0,82 \%$ \\
\hline RSAD2 & $\begin{array}{l}\text { radical S-adenosyl } \\
\text { methionine domain } \\
\text { containing } 2 \\
\end{array}$ & 91543 at & 91543 & 2,67 & 7,794188724 & $1,61 \mathrm{E}-05$ & $0,22 \%$ \\
\hline FN1 & fibronectin 1 & 2335 at & 2335 & 2,68 & 7,430931476 & $7,88 \mathrm{E}-05$ & $0,54 \%$ \\
\hline OAS1 & $\begin{array}{l}\text { 2',5'-oligoadenylate } \\
\text { synthetase } 1,40 / 46 \mathrm{kDa}\end{array}$ & 4938_at & 4938 & 2,70 & 9,344873561 & $5,70 \mathrm{E}-06$ & $0,13 \%$ \\
\hline DDX60L & $\begin{array}{l}\text { DEAD (Asp-Glu-Ala-Asp) } \\
\text { box polypeptide } 60 \text {-like }\end{array}$ & 91351 at & 91351 & 2,72 & 8,058742044 & $1,48 \mathrm{E}-06$ & $0,08 \%$ \\
\hline DDX60 & $\begin{array}{l}\text { DEAD (Asp-Glu-Ala-Asp) } \\
\text { box polypeptide } 60\end{array}$ & 55601 at & 55601 & 2,79 & 8,523992857 & $6,16 \mathrm{E}-06$ & $0,13 \%$ \\
\hline OAS3 & $\begin{array}{l}\text { 2'-5'-oligoad enylate } \\
\text { synthetase } 3,100 \mathrm{kDa}\end{array}$ & 4940_at & 4940 & 2,80 & 8,997755199 & $1,89 \mathrm{E}-06$ & $0,09 \%$ \\
\hline CXCL12 & $\begin{array}{l}\text { chemokine ( } \mathrm{C}-\mathrm{X}-\mathrm{C} \text { motif) } \\
\text { ligand } 12\end{array}$ & 6387 at & 6387 & 2,81 & 8,014471187 & $8,32 \mathrm{E}-08$ & $0,02 \%$ \\
\hline SCNN1A & $\begin{array}{l}\text { sodium channel, } \\
\text { nonvoltage-gated } 1 \text { alpha }\end{array}$ & 6337 at & 6337 & 2,89 & 7,688196305 & $3,01 \mathrm{E}-08$ & $0,02 \%$ \\
\hline RET & ret proto-oncogene & 5979_at & 5979 & 2,98 & 8,368634107 & $1,88 \mathrm{E}-07$ & $0,03 \%$ \\
\hline IFIT3 & $\begin{array}{l}\text { interferon-induced protein } \\
\text { with tetratricopeptide } \\
\text { repeats } 3\end{array}$ & 3437 at & 3437 & 3,06 & 6,634329881 & $5,65 \mathrm{E}-08$ & $0,02 \%$ \\
\hline ANXA1 & annexin $\mathrm{A} 1$ & 301 at & 301 & 3,10 & 6,803049914 & $6,22 \mathrm{E}-05$ & $0,48 \%$ \\
\hline LOC402778 & $\begin{array}{l}\text { CD225 family protein } \\
\text { FLJ76511 }\end{array}$ & 402778_at & 402778 & 3,12 & 7,506900476 & $3,35 \mathrm{E}-06$ & $0,10 \%$ \\
\hline SAMD9 & $\begin{array}{l}\text { sterile alpha motif domain } \\
\text { containing } 9\end{array}$ & 54809_at & 54809 & 3,24 & 8,386274014 & $1,19 \mathrm{E}-06$ & $0,07 \%$ \\
\hline MMP2 & $\begin{array}{l}\text { matrix metallopeptidase } 2 \\
\text { (gelatinase } A, 72 \mathrm{kDa} \\
\text { gelatinase, } 72 \mathrm{kDa} \text { type IV } \\
\text { collagenase) }\end{array}$ & 4313_at & 4313 & 3,31 & 7,228038706 & $3,45 \mathrm{E}-06$ & $0,10 \%$ \\
\hline OAS2 & $\begin{array}{l}\text { 2'-5'-oligoad enylate } \\
\text { synthetase } 2,69 / 71 \mathrm{kDa}\end{array}$ & 4939_at & 4939 & 3,32 & 9,504688775 & $1,18 \mathrm{E}-04$ & $0,69 \%$ \\
\hline PRSS23 & protease, serine, 23 & 11098 at & 11098 & 3,64 & 7,832283105 & $1,20 \mathrm{E}-06$ & $0,07 \%$ \\
\hline IFI44 & interferon-induced protein 44 & 10561 at & 10561 & 3,78 & 6,601749899 & $1,89 \mathrm{E}-07$ & $0,03 \%$ \\
\hline IFIT1 & $\begin{array}{l}\text { interferon-induced protein } \\
\text { with tetratricopeptide } \\
\text { repeats } 1\end{array}$ & 3434_at & 3434 & 3,86 & 9,039040561 & $8,79 E-06$ & $0,15 \%$ \\
\hline IFI44L & $\begin{array}{l}\text { interferon-induced protein } \\
\text { 44-like }\end{array}$ & 10964_at & 10964 & 4,22 & 8,988775342 & $5,30 \mathrm{E}-06$ & $0,12 \%$ \\
\hline SPARC & $\begin{array}{l}\text { secreted protein, acidic, } \\
\text { cysteine-rich (osteonectin) }\end{array}$ & 6678 at & 6678 & 4,53 & 8,432603952 & $6,74 \mathrm{E}-06$ & $0,14 \%$ \\
\hline
\end{tabular}




\section{Curriculum Vitae}

Elke Ziegler

02.10.1981, born in Darmstadt, Germany

Papenhöhe 14, 25335 Elmshorn, Germany

elke.ziegler@gmail.com

\begin{tabular}{ll} 
Professional experience \\
\hline $2009 / 07-2012 / 12$ & $\begin{array}{l}\text { Universtätsmedizin Göttingen } \\
\text { Scientific assistant }\end{array}$ \\
\hline $2008 / 05-2009 / 05$ & $\begin{array}{l}\text { Rheinische Friedrich-Wilhelms-Universität Bonn } \\
\text { Scientific assistant }\end{array}$ \\
\hline $2007 / 03-2008 / 03$ & $\begin{array}{l}\text { Sonnen-Apotheke Wolfhagen } \\
\text { Pharmacist }\end{array}$ \\
\hline Education & \\
\hline $2009 / 10-2012 / 09$ & $\begin{array}{l}\text { Georg-August-Universität Göttingen } \\
\text { International PhD program Molecular Medicine }\end{array}$ \\
\hline $2010 / 09-2012 / 07$ & $\begin{array}{l}\text { Verwaltungs- und Wirtschaftsakademie Kassel } \\
\text { Graduation: Economist in Health Care } \\
\text { (Gesundheits- und Sozial-Ökonomin (VWA)) }\end{array}$ \\
\hline $2008 / 04-2009 / 03$ & $\begin{array}{l}\text { Rheinische Friedrich-Wilhelms-Universität Bonn } \\
\text { Graduation: Degree in Pharmaceutics } \\
\text { (Diplom-Pharmazeutin) }\end{array}$ \\
\hline $2001 / 10-2005 / 09$ & $\begin{array}{l}\text { Johann Wolfgang Goethe-Universität Frankfurt/Main } \\
\text { Graduation: License to practice Pharmacy } \\
\text { (Approbation) }\end{array}$ \\
\hline $1992-2001$ & $\begin{array}{l}\text { Liebfrauenschule Bensheim, Gymnasium } \\
\text { Graduation: Abitur }\end{array}$
\end{tabular}

